0

Mirjam Grewe-Salfeld

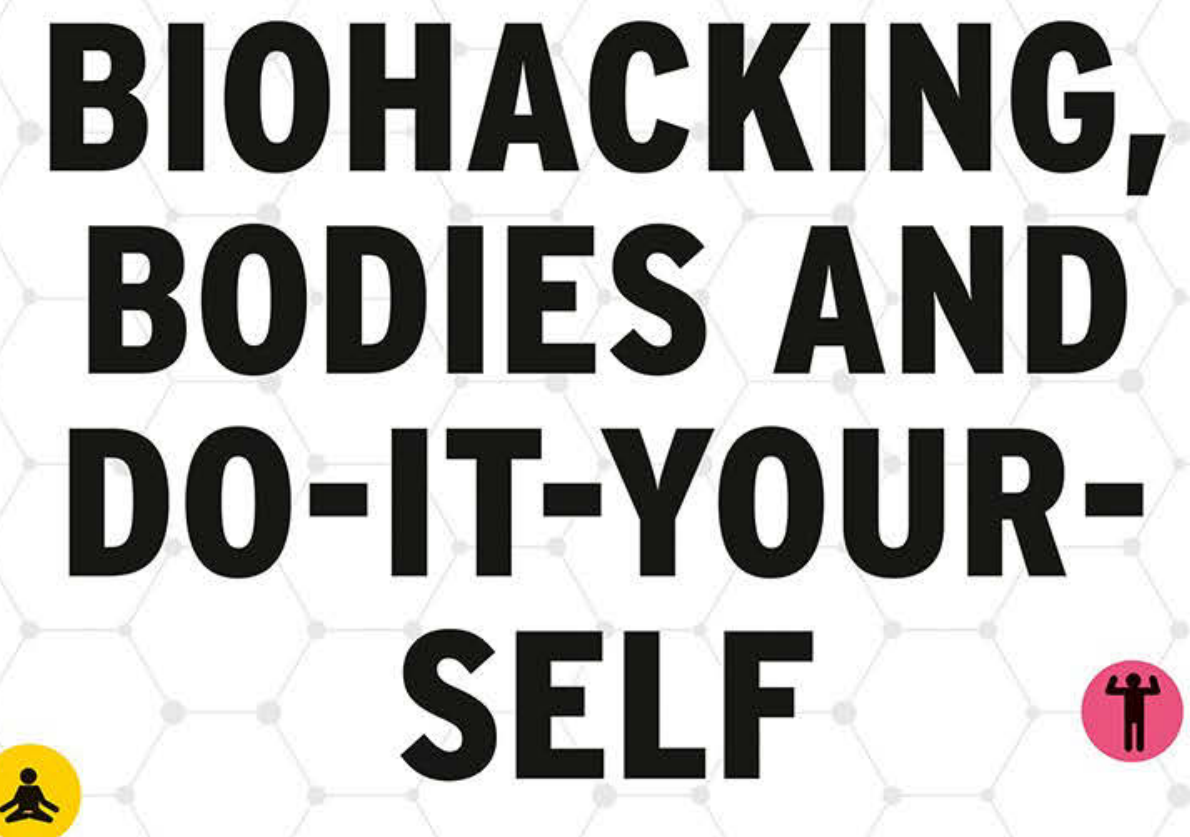

The Cultural Politics of Hacking Life Itself
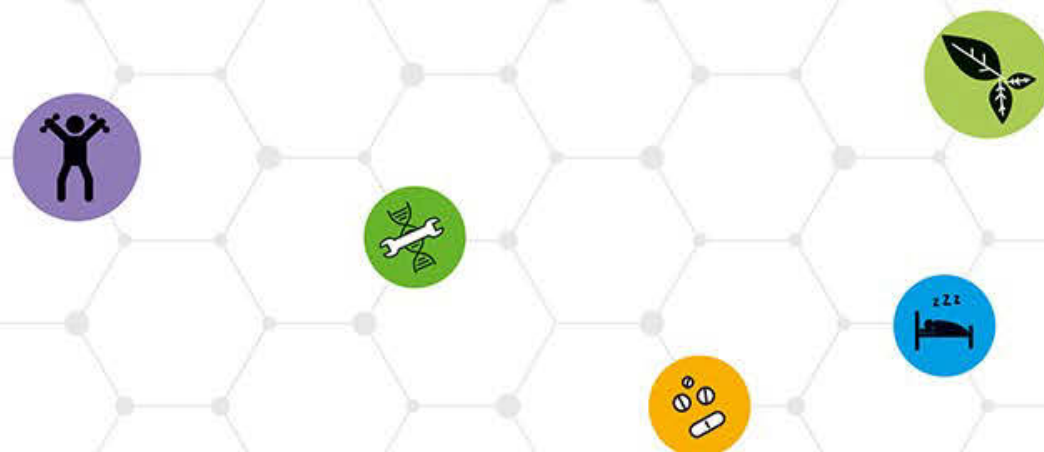

[transcript] A merican Culture studies 
Mirjam Grewe-Salfeld

Biohacking, Bodies and Do-It-Yourself

American Culture Studies | Volume 36 
Mirjam Grewe-Salfeld, born in 1991, is a researcher in literary- and cultural studies. She received her PhD from the Department of American Studies at Universität Potsdam. Her research interests include the cultural representation and history of medicine, body studies and biotechnology. 
Mirjam Grewe-Salfeld

\section{Biohacking, Bodies and Do-lt-Yourself}

The Cultural Politics of Hacking Life Itself

[transcript] 
This work has been accepted as a PhD thesis at the University of Potsdam, Faculty of Arts, in 2020 under the title "Do-It-Yourself - Biotechnology and the New Cultural Politics of Life in the Contemporary United States". The PhD thesis was supervised and reviewed by Prof. Dr. em. Rüdiger Kunow and Prof. Dr. Marc Priewe.

\section{Bibliographic information published by the Deutsche Nationalbibliothek}

The Deutsche Nationalbibliothek lists this publication in the Deutsche Nationalbibliografie; detailed bibliographic data are available in the Internet at http://dnb.d-nb.de

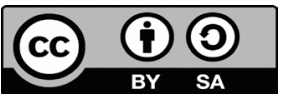

This work is licensed under the Creative Commons Attribution-ShareAlike 4.0 (BY-SA) which means that the text may be remixed, build upon and be distributed, provided credit is given to the author and that copies or adaptations of the work are released under the same or similar license. For details go to

http://creativecommons.org/licenses/by-sa/4.o/

Creative Commons license terms for re-use do not apply to any content (such as graphs, figures, photos, excerpts, etc.) not original to the Open Access publication and further permission may be required from the rights holder. The obligation to research and clear permission lies solely with the party re-using the material.

\section{First published in 2022 by transcript Verlag, Bielefeld (C) Mirjam Grewe-Salfeld}

Cover layout: Maria Arndt, Bielefeld

Printed by Majuskel Medienproduktion $\mathrm{GmbH}$, Wetzlar

Print-ISBN 978-3-8376-6004-3

PDF-ISBN 978-3-8394-6004-7

https://doi.org/10.14361/9783839460047

ISSN of series: $2747-4372$

eISSN of series: $2747-4380$

Printed on permanent acid-free text paper. 


\section{Contents}

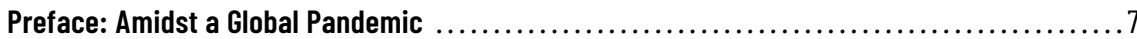

\section{PART I: INTRODUCTIONS}

1 Introduction: Biotechnologies, Bodies and Biomakers .......................... 17

2 Biology and Culture - an Overview of the Field .............................. 33

\section{PART II: BACKGROUND}

3 The Promise of Small Things - The Cultural Presence of (Molecular) Biology ......... 75

4 With my Own two Hands - Tracing DIY from Individualism to Maker Culture ........... 97

\section{PART III: 'HACKING' LIFE ITSELF}

$5 \quad$ 'Hacking' Life Itself - In Pursuit of a Definition ................................ 129

$6 \quad \#$ iambulletproof - A Layperson's Guide to Self-Enhancement ...................... 137

$7 \quad$ A Pill for Every III - Drugs and Supplements ................................ 155

8 Test Yourself - Making the Invisible Visible ................................. 175

9 Homo Technologicus? - The Technological Self, Inside Out ...................... 197

10 "The Human Need to Fiddle" - Tinkering with Technology ........................ 217 


\section{PART IV: REFLECTIONS}

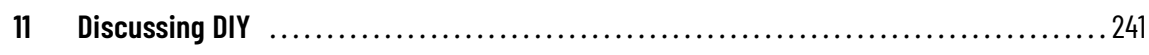

12 Conclusion: Between Politics and Promise ............................... 281

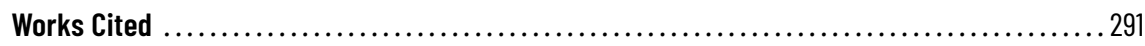




\title{
Preface: Amidst a Global Pandemic
}

\begin{abstract}
"Who can use the term 'gone viral' now without shuddering a little? Who can look at anything any more - a door handle, a cardboard carton, a bag of vegetables - without imagining it swarming with those unseeable, undead, unliving blobs dotted with suction pads waiting to fasten themselves on to our lungs?
\end{abstract}

Who can think of kissing a stranger, jumping on to a bus or sending their child to school without feeling real fear? Who can think of ordinary pleasure and not assess its risk? Who among us is not a quack epidemiologist, virologist, statistician and prophet??"

Arundhati Roy, "The Pandemic is A Portal," 03.04.2020

The following book is a revised version of my $\mathrm{PhD}$ thesis, which I handed in for review in February 2020. While finalizing it, I included a footnote on this 'new' virus in China and the biology-based discrimination that soon followed it around the world. \#iamnotavirus was trending around that time and I felt it would only be fitting to include this entanglement of biology and culture at least as a side remark. When rereading my thesis prior to defending it in November 2020, I had to laugh out loud and shake my head. Little did we know that life would change dramatically - that we would all become biological risks to each other. That life as we knew it would indeed stand still.

A global pandemic, endless quarantining, biologists and epidemiologists becoming new superstars, discourses of risks around the clock, national health measures, increasing skepticism of science, medical headlines each and every day. Who knew that our life could be so...biological? As a lot of scholarship in the medical humanities, in science and technology studies, in biocultures has already demonstrated: Our lives have always been 
biological. This pandemic has only brought it to the forefront, to the headlines, once more and with a vengeance. SARS-CoV-2 has highlighted our "biological citizenship” (Rose and Novas) with an intensity we did not expect. It forced us to confront our biological risks and to try to understand them. It has shown us that globalization truly knows no borders and limits, that we are deeply intertwined with all that surrounds us, no matter how small or seemingly insignificant.

While preparing my thesis for publication, 'the pandemic' has had its first anniversary: vaccinations programs have started (mainly in rich countries), mutations have occurred that seem to make 'the virus' more dangerous and more effective (based on our current knowledge), and countries still try to grapple with how to best address this challenge and contain new waves of infections (with differing success). Many of us have been 'at home' for a prolonged amount of time now, have not seen friends and loved ones, have not been able to say goodbye or hello; jobs have been lost, livelihoods threatened or destroyed, new burdens shouldered. This preface wants to set some of the biocultural discussions in my dissertation into this new context, this new situation. And it wants to highlight how 'do-it-yourself' (DIY) biology and medicine is present also amidst a global pandemic, as first responses, creative solutions and political activism.

SARS-CoV-2 has demonstrated how much force the biological has over culture, society, politics, and the economy, on a global scale. As the introductory quote by Indian novelist Arundhati Roy illustrates, for most of us biology has become deeply ingrained into our everyday life - biology has come to the forefront of our daily actions and interactions during the last year. But the pandemic, as Andy Horowitz writes, is not just biological but also cultural: "The history that scholars will come to name the Covid-19 pandemic' ultimately will have as much to do with the social world the virus encountered as it does with the virus itself." (n.p.) This pandemic makes the inherent connection between the world of biology and the world of culture blatantly obvious - a deep entanglement that Chapter 2 of this book discusses in more detail. This is not just true on a more philosophical or abstract level but also on a very concrete one: During the course of the Covid-19 pandemic the role of pop-cultural media for science communication and the dissemination of facts and knowledge has become even more prominent. Not just the virus, its biological properties, daily infections or biopolitical measures are constantly reported on, but also epidemiologists and other experts have become new media personalities. In "Epidemic Entertainments" Nancy Tomes claims that diseases and epidemics have gained a new cultural visibility due to modern communication media and the new globalized threat they pose (626). Ever since the early $20^{\text {th }}$ century with its parallel rise of mass media and germ theories of disease - accounts of viruses, germs and epidemics have fascinated, scared and educated people. This pandemic is no exception. One of the first examples of 'pandemic productions,' as we could call them, is the $17^{\text {th }}$ season of the medical drama Greys Anatomy, which was among the first shows to directly address the pandemic and its reality in hospitals. Instead of the usual dramatic surgeries and exceptional cases, the fictional doctors now primarily care for Covid patients and their work is dominated by safety protocols and personal protective equipment (PPE). These imaginary, fictive engagements give faces and voices to an ominous mass of healthcare workers struggling at the frontlines of the pandemic - applauded and neglected at the same time. Similar, 'real-life' attempts can be found on social me- 
dia with images of healthcare workers in PPE or after intensive PPE use, physically and mentally collapsing under the sheer burden placed on them.

Stories of disease, Tomes claims, are not just entertaining but also educational: In news media accounts and journalism, stories of disease serve to perpetuate scientific facts out of the laboratory and clinic into the households of the masses (643). They become media of mass education as well as mass responsibilization. As such, popular media have the potential to increase the scientific literacy of their consumers: scientific and biological facts are presented, explained and discussed - from the biology of the virus to scientific estimates and projections about the course of the pandemic, to different types of vaccines and their working mechanism (cf. Ch. 2). However, especially in the public and political climate of a deeply divided American Presidential Election Year, Covid-19 has made the rifts in American society even more apparent: A considerable number of people do not seem to believe in science and disregard political guidelines; skepticism and conspiracy theories about the origin of the virus, political measures or vaccines abound - and this trend of Covid Deniers is not limited to the US. In an overflow of information also misinformation flourishes. Between all the facts, data and opinions presented on (social) media, it becomes hard to distinguish claims, to determine what is right and wrong, contextualize, evaluate sources, especially if people are not "scientifically literate" (cf. Ch. 2.1). Like a magnifying lens, Covid has amplified tendencies such as alternative facts or conspiracy theories and at the same time given them a higher urgency, when sticking to rules and trust in scientific knowledge and guidelines can help contain the virus and save lives.

The 'viral spread' - in the positive and negative sense - also in culture demonstrates that this pandemic is as much cultural, social, political as it is biological. "Just as people's so-called 'pre-existing conditions' shape their individual vulnerability to Covid19," Horowitz writes, "social pre-existing conditions shape the course of the pandemic broadly." (n.p.) Reminiscing on the nature of the virus, Soraya de Chadarevian and Roberta Raffaeta argue that this pandemic is another instance that demonstrates how the social and biological world are inextricably connected (5). I would suggest that also this contingency, the inability to disconnect the virus from our social activities and daily life, our culture and economy, heightens our perceived vulnerability to this new danger. What the pandemic has demonstrated is that we are all biological, as mundane as that might sound. Each person we meet has become a biological risk factor, leaving us vulnerable even where we previously have felt safe - at home, in our communities, with families and friends. During the course of all this, we have been faced with new choices to navigate, which might directly impact our own health and that of our loved ones but more indirectly also impact our healthcare systems and our society's ability to cope with such a crisis. Covid-19 intensified our perceived and experienced biological vulnerability.

The result of this vulnerability is also anger, hate, discrimination, and racism, which leaves some bodies in even greater states of vulnerability. Anti-Asian hate crimes and discrimination have led to a social media outcry under the hashtag \#iamnotavirus already during the first few weeks of the pandemic and they have continued even as the hashtag lost its allure and moved out of the news cycle. Especially in the US, against the backdrop of Black Lives Matter protests against police violence, Covid-19 has also 
become a matter of race. Covid is not "a great equalizer" as it has been proclaimed by some. Instead, intersectional approaches show how some people are at greater risks. As Clyde W. Yancy writes, increased risk is attributable not only to (individual) biological risk factors, but also to socio-economic realities and "pre-existing" health care disparities. Collected data have shown that especially ethnoracial minority groups face a higher disease burden, which could be attributed to different causes: they tend to be employed under precarious conditions, belong to low-income communities, reside in higher density living arrangements, lack access to health care or health insurance, or suffer from more pre-existing medical conditions and comorbidities, also as a result of their social and economic conditions (Berkhout and Richardson 51).

In scholarly responses to the pandemic, references to Foucault and his notion of biopolitics abound. And it is certainly true: Lockdowns, wearing face masks, practicing social distancing, reducing contacts, staying at home, washing hands in the name of \#flattenthecurve. These containment measures are all biopolitical strategies aimed to facilitate life, in which citizens are called upon to act responsibly towards the self and the whole population. As Malcom Brady argues rules have power when they either make sense to us individually and collectively (then they become institutionalized and normalized) or when they are enforced through fear or force (3-4). Both have been at play in this pandemic: Governments and states have increased surveillance measurements and managed their population to a greater degree (Sylvia 3). Especially surveillance technologies such as contact tracing apps and the lack of data security they provide have been at the center of attention and debate in this context. ${ }^{1}$ At the same time, people are tasked with taking more and more responsibility for their own health and their own decisions - a trend that the pandemic has made more visible and concrete for many people (Sylvia 3).

In general, biopolitical strategies, as they were used also in response to the pandemic, often are debated controversially. Their connotations also in scholarly discussions are mostly negative: They are argued to be disciplinary, state-controlled, coercive, peer-controlled measures of surveillance, 'biopolitics of control' and top-down regulations of everyday life and personal freedoms. And while all these perceptions are certainly true and should not be neglected, I also want to reverse the idea of control and instead think of biopolitics of care that were visible in the first months of the pandemic. Such caring biopolitics are motivated by an ethics of empathy, care, and solidarity. They were located not solely in government restrictions, but in community acceptance, an "affective atmosphere" (B. Anderson) of cohesion and belonging: "We are all in this together, let's see how we can help and get through this!" Paroles to close ranks and hold on were invoked to protect more vulnerable populations against this new and unknown 'enemy.' In Germany and other European countries, this spirit was visible in the beginning, motivated people to endure new restrictions to their individual freedom - and it worked. 


\section{'Hacking' Covid-19}

The 'biopolitics of care,' the vague urge to act and help, turned into something very concrete, material, in grassroots responses aiming to 'hack' the pandemic and our responses to it. During the first months of the pandemic, Cindy Khotala writes, the global maker communities' response was fast, "a surge of enthusiasm and a rush to printers" (n.p.). When many countries reported a lack of PPE for health care staff - and even less for 'ordinary' citizens - people sprang into action and organized DIY alternatives. Annika Richterich looks at how UK hacker and maker communities responded to these shortages of healthcare equipment during the first wave of the pandemic using opensource design, laser cutters, 3D printers, and sewing machines. The lack of PPE brought maker communities into conversation with health professionals with the aim of developing and producing urgently needed supplies, such as face shields/visors and scrubs, masks and mask strap holders, or even "aerosol boxes" for intubation of Covid patients (161). Their work, as in many countries, was coordinated directly with affected healthcare professionals, responding to their individual needs. ${ }^{2}$ From my experience, this surge of collaboration and community work did not stop with those people who would consider themselves as part of a (somewhat formalized) maker community: Sewing patterns for DIY masks were distributed widely on the internet and in mainstream media, supplies such as elastic straps were in high demand (at least in Germany). Before commercial production could start, self-made masks were produced for personal use or donated to health care facilities. As the New York Times reported, "makers" around the world organized online to discuss, collect, evaluate and distribute all sorts of instructions: from masks and face shields to hand sanitizer and ventilator (parts) (Petri). For Richterich such practices are also a form of "critical making," a political response to a politics of austerity in the UK health care system, in which making as a type of activism tries to make up for systemic problems and political mismanagement in healthcare sectors (160). Such practices, she also contends, are not new to this crisis but have been part of DIY health and wellbeing movement in different guises (162). Indeed, some of the practices I discuss throughout this book are exactly such types of activist responses to ethical dilemmas, problematic distributions of access and power.

The „citizen-biotech-economies" (Meyer, "Build") that feed into and are the result of the rise of DIY biology and maker spaces have also provided tools and spaces for different types of DIY practices in responses to the recent pandemic: For example, in May 2020 the MIT Technology Review reported on Ian Hilgert-Martiszus, a former lab technician and programmer, who had done a DIY trial on Covid-19 antibodies among 40 of his friends and acquaintances. He and his test subjects wanted to know whether the colds, fevers, and runny noses they had experienced in the past few months were Covid19 symptoms. In March, Hilgert-Martiszus bought kits to test human blood serum from a Chinese supplier and began testing. As a lab technician he knew what he had to do and was able to borrow some of the equipment from a nearby university. However, he says „I

2 Of course, these practices of making were also faced with practical and ethical problems, such as existing regulations for health products. Are DIY solutions as effective as tested products? cf. Richterich 163. 
am just doing it at home. This is total citizen science." (Regalado, "This man assembled") In April he posted his results and set-up online - before any of the big medical centers did so: putting his DIY effort, for a short while at least, into the "forefront of the search for antibodies" (Regalado, "This man assembled").

But also states and governments made use of maker approaches and the willingness of citizens and societies to participate. Hackathons were organized to deal with the after-effects of the pandemic, to address pressing issues from the perspectives of citizens or to design technologies that help to counter new, rising waves of infection. One example of state-led interventions with DIY methods are reports on a government study in the US: As the LA Times and US News reported, during the summer of 20206,000 families were taking part in a large study to find out how kids and teens are affected by the virus and answer the pressing concern of transmissions between kids and adults with possible consequences for the opening of schools, daycare and so forth. The participants, however, were not examined by doctors but performed DIY nasal swaps twice a week in their own home. They received testing supplies via mail, answered surveys and collected their own specimen: from nasal swaps to blood and stool samples. Their homes became medical test centers. In the articles, one participant in this governmentfunded study explained: "We were excited to be able to feel like we could contribute somehow [...] This virus is so unknown. Any little bit we can do felt like we were doing something to help." (L. Tanner) Through their participation in this study, I would argue, they also performed their biological citizenship: They voluntarily and eagerly self-educate themselves and integrate biological knowledge and testing into their everyday life. They donated their biological material for the community at large, for the greater good. It was their responsibility, as good biological citizens, to do what they can to help.

Many people in the maker movement knew and hoped that their solutions would be used as a last resort, an alternative in case of need and not a replacement of tried and tested materials. Still, the flurry of action and fast development of DIY solutions have tangibly demonstrated the strength of open-source principles and community-based work. The fact that so many people responded to this biological crisis with hands-on action, the striking wave of (more mundane) DIY activities during the first months of stay-at-home orders and quarantining have made 'making' and DIY visible as a trend once more. In times of crisis, making has drawn people together to create community and show solidarity, against this invisible new danger to everyone.

\section{Appreciation}

On a personal note, faced with the unknown and uncontrollable in hindsight I am very grateful for these first few months of collective action and solidarity that the pandemic has brought us. Before there was time to politicize, it was time to act, make and make do. I want to use this space to also thank some of the people involved in the 'making' of this book. Where to begin and where to end? I could go back in time and thank my parents, who bought me endless shelves of books as a child, fueling my love of words and stories; or even Grey's Anatomy for sparking a brief desire to become a doctor as a youth, fueling my interest in the culture and representation of medicine. This book, 
however, would not have been possible without the encouragement of my dissertation advisor, Rüdiger Kunow, who not only inspired me to take on this project, but patiently read through the hundreds and hundreds of pages I gave him and provided valuable feedback and notes. His hints to shorten drafts were mainly executed unsuccessfully on my part. Sorry, dear reader! Thanks is also due to Marc Priewe, the second advisor, who provided important insights where I could be a bit more critical and less enthusiastic. The organizers and participants of a postgraduate conference in Mannheim Life Writing in the Digital Age: Quantification, Optimization and the Self (Sept. 2017) have provided valuable feedback on my thoughts about personal genomics and optimization, and the organizers and participants of the $15^{\text {th }}$ Annual Spring Academy at the Heidelberg Center for American Studies (March 2018) have critiqued a first draft of Chapter 4, providing new input on ideas and ideals of individualism in American society. Thank you to Juliane Straetz for our feedback and discussion session on the overall outline of the thesis! My dear proofreaders should also not go unrecognized, especially Jochen Salfeld, who commented from the standpoint of a molecular biologist and researcher as well. The members of the examination committee, Nicole Waller (Universität Potsdam), Rüdiger Kunow (Universität Potsdam), Marc Priewe (Universität Stuttgart), Marie-Luise Raters (Universität Potsdam), and Monika Pietrzak-Franger (Universität Wien), deserve thanks for their insightful questions and comments on the finalized version - and the latter also for sparking my interest in the Medical Humanities in the first place. During my bachelor studies she told me that she would also read my PhD thesis (if it ever came to that), I remembered and so she did! Without the unwavering support of my family and friends this whole project would not have been possible. Thank you to my partner, who at times has been a bigger champion for this book than I have, and our daughter, who has taught me a new outlook on life and how it should be lived! And last but not least, back to my parents, who urged me to at least do something during a time of uncertainty and confusion - this something led to this!

There has been a rush to respond to the Covid crisis also in many academic fields - and I am certainly no exception to that rush: "The need to both understand and to contribute is pressing, even while we too experience exhaustion, fear, loneliness and grief. There is a tension between the sense of exigency, to step up and act, and the need to withdraw and protect our own." (Khotala). Many of these responses, I am sure, are also personal projects of 'making sense,' of contributing somehow to public sense-making processes. In this sense, attentive readers will find some references to the Covid-19 pandemic spread throughout the pages and footnotes of this book. Almost every topic of interest that I will discuss can be related to this acute biological crisis, directly or indirectly. That tells me they are here to stay. What leaves me wondering is what our world post-Covid will look like? Will it differ from the past, and if so, how? How soon will our memories of this very embodied, very biological time start to fade? What can we take and learn from this experience? As Arundhati Roy writes, the Covid pandemic has managed to bring the engines of capitalism to a halt, possibly buying us time to determine whether or not we want to fix it or "look for a better engine:"

"Our minds are still racing back and forth, longing for a return to 'normality', trying to stitch our future to our past and refusing to acknowledge the rupture. But the rup- 
ture exists. And in the midst of this terrible despair, it offers us a chance to rethink the doomsday machine we have built for ourselves. [...] Historically, pandemics have forced humans to break with the past and imagine their world anew. This one is no different. It is a portal, a gateway between one world and the next. We can choose to walk through it, dragging the carcasses of our prejudice and hatred, our avarice, our data banks and dead ideas, our dead rivers and smoky skies behind us. Or we can walk through lightly, with little luggage, ready to imagine another world. And ready to fight for it." -- Arundhati Roy, "The Pandemic is a Portal", 30.04.2020

One year in, I am not certain if I share her optimism about the possibilities opened up by this pandemic - but I sure hope she is right.

Potsdam, 30.04.2021 


\section{PART I: INTRODUCTIONS}





\title{
1 Introduction: Biotechnologies, Bodies and Biomakers
}

\author{
Dr. Eli Mays: "I am a biohacker. We're about \\ the democratization of science. The CIA is \\ not." \\ Agent Hannah Wells: "For a reason. Bio- \\ engineering in the right hands, great, In the \\ wrong hands..." \\ Dr. Eli Mays: "Exactly. You know gene editing \\ is listed as a weapon of mass destruction, \\ right? You guys aren't focused nearly enough \\ on bioterror." \\ Agent Hannah Wells: "That's what I have \\ been working on." \\ Dr. Eli Mays: "Mutually-assured destruction \\ is passé. Now it's a biorogues' world. If you \\ don't hold people to account, shit will hap- \\ pen. You need to be monitoring everyone. \\ Like I do."
}

(Designated Survivor, \#privacyplease 3.02, 19:00-21:31)

The third season of Designated Survivor (Netflix, 2019) saw a new threat to the safety of the American nation: bioterrorism, and not just any kind. Rogue scientists, making use of the growing do-it-yourself (DIY) biology movement and CRISPR technology, had engineered a virus designed to target only people of color in an effort to sterilize them - biologically engineered racism and voter suppression. Agent Hanna Wells, who in the previous seasons was uncovering a variety of terrorist plots against the US, seeks out Dr. Eli Mays, a DIY biologist, and starts to work with him to uncover this dark scheme. Their search leads them into the DIY biology movement, its secret and not-so-secret labs, practitioners and practices - discussing questions of access and democratization, threats and dangers, regulation and safety - until in "\#scaredsh"tless" (Episode 8) the president is notified of this imminent danger and it becomes a political 
crisis. Bioterrorism of this kind, he and his staff note, is nothing they had to deal with before because the technology simply did not exist: "Until now. For a couple of thousand bucks, an amateur scientist can produce a plague that could wipe out all of humanity" (Ep. 8, 04:10-4:17). Of course, the threat is prevented, the suspect apprehended, and the political plot uncovered, but not before the "rogue biologist" was able to upload his design in DIY forums for all to access. The only way to contain such threats in the future, the "DIY guy" (12:15) Mays notes, is to regulate the sale of deadly genetic sequences and hire scientists like him to advice and work with the government. Genetic engineering, "CRISPRed" bacteria and viruses, unregulated science in and outside of registered laboratories here is turned into the stuff of nightmares - an imminent threat to the safety of large parts of the American population. ${ }^{1}$

This plot, however, is not the only way in which DIY biology and the contexts of its formation enter the narrative: genetic testing for disease and heritage and its repercussions - even a secret DNA test without the person's knowledge or consent - form an important part of some of the storylines, while the Opioid Crisis, high drug prices and a fight against 'Big Pharma,' as well as differences in access to healthcare are dealt with as political issues. ${ }^{2}$ This cultural representation demonstrates how ubiquitous and contested practices of DIY biology and medicine have become. But more than merely inserting them into the narrative, Designated Survivor also discusses some of their drawbacks and limitations: the repercussions of access for all, potential abuses of technologies, differential distribution of resources, slippery slopes into racism and eugenics, the need for regulation, the power of (pharmaceutical) companies and the real-life experiences of many Americans. It demonstrates that some of these practices have become mundane and expected - genetic testing for example - while others are new and dangerous - the hacking of and tinkering with DNA. The series lets us know: Biohacking, in different guises, is omnipresent in American culture and society.

In August 2020, another show, Biohackers, opens with a similar fear of bioterrorism: The first sequences show a train compartment full of passengers who collapse one

CRISPR/Cas-9 (from Clustered Regularly Interspaced Short Palindromic Repeats), often also called 'gene scissors' in colloquial use, is a technology that allows scientists to cut DNA at any desired location, so that they can edit in new strands of DNA, thereby inserting new information into the genome. When first developed and used (2012-2015), CRISPR represented a minor revolution in gene editing technology, earning scientists Emmanuelle Charpentier und Jennifer Doudna a Breakthrough Prize in Life Sciences 2015.

2 One character's wife is in and out of rehab for her fentanyl addiction, leading him to start a political campaign against the manufacturers of the drug, forcing them to provide their newest invention - a drug to counter the effects of addiction - to addicts for free. The high prices for insulin and the resulting lack of treatment or rationing of doses by people with diabetes is targeted in Ep. 3 ("\#privacyplease"), inserted into the narrative also via clips from real-life Americans that talk about their problems, reflecting real-life issues in fiction. A political opponent's DNA is taken from a spoon and analyzed without his consent and the results, a high likelihood of Alzheimer's disease, leaked to the press. One political advisor tests herself for cancer mutations to learn whether she is likely to develop cancer like her mother. The communications director has done a genetic test to find his birth parents and finds a child he fathered as a sperm donor because his genetic data are in the company's databases. In one episode a refugee child's medical treatment in a US hospital highlights the difference in access to and quality of medical care between the US and Guatemala. 
after the other - without apparent reasons. Later on, we learn that genetically engineered mosquitos were released on that train. ${ }^{3}$ And those mosquitoes were the result of illegal and unregulated experiments in private laboratories. The mini-series, a German production published world-wide on the streaming service Netflix in August 2020, introduces the viewer to the world of DIY biology through the eyes of a young medical student, Mia Akerlund, whose personal history lets her investigate the well-known biologist Prof. Dr. Tanja Lorenz. The story is a revenge and coming-of-age plot set in a backdrop of biohacking and synthetic biology. ${ }^{4}$ Science that is and cannot be performed in universities is in this series performed in home- and private laboratories. This includes at home in a shared flat, in a DIY lab with second-hand equipment, or at a high-tech personal lab. In these spaces, Biohackers represents a plethora of DIY biology practices in different guises. The more 'high-end' biohacking portrays in the show are self-made genetic treatments for Huntington's disease and the aforementioned synthetically engineered mosquitos. At the same time, subplots also explore others forms of DIY biology and those are often more 'flashy' science: fun tinkering with biological materials is used to create fluorescent marihuana plants or a bio-piano; bloody examples of body hacking with crude devices portray a form of biohacking focusing on the human body and its potential for optimization. ${ }^{5}$

If these two examples sound too abstract, too much like (science) fiction, consider the following: In June 2019 Sigal Samuel published an article on vox.com, in which he answers nine questions about biohacking "you were too embarrassed to ask." His article is one of a series of articles online that aim at explaining the phenomenon of biohacking to their audiences. ${ }^{6}$ Samuel writes:

Even if you haven't heard the term "biohacking" before, you've probably encountered some version of it. Maybe you've seen Twitter CEO Jack Dorsey extolling the benefits of fasting intermittently and drinking 'salt juice' each morning. Maybe you've read about former NASA employee Josiah Zayner injecting himself with DNA using the gene-editing technology CRISPR. Maybe you, like me, have a colleague who's had a chip im-

3 Of course, the heroin of the story is able to save everyone, but still: In the face of a global pandemic, the vivid images of collapsing people and doctors in hazmat suits was probably one of the reasons why Biohackers' release was pushed back from March 2020 to August 2020.

4 A review for the journal Science concludes that - while sometimes compressed for dramatic purposes - the science itself is mostly portrayed accurately and offers insights into biological and scientific processes (Greenbaum). It also highlights that Biohackers delves into some of the ethical, legal and social concerns around biohacking and could thus be used as a "pedagogical vehicle." Greenbaum summarizes that based on what is shown in the series, regulation might not be a bad idea - and in fact, the biology that is represented here would be illegal under Cerman regulations, at least in spaces in which it is performed.

5 This creative side of biohacking is mostly represented by the protagonists two roommates - whose characterization, however, primarily consists of being stereotypical 'nerds' or 'geeks,' with one of them embodying practices of bio-art and the other a Dave Asprey, Dangerous Things-type of bodyhacker when he inserts chips and magnets into his own body or creates eye-drops that let you see in the dark (for more on Asprey's Bulletproof Franchise, see Ch. 6; the biohacking company Dangerous Things is analyzed in Ch. 9).

6 Similarly, Jewell and Weatherspoon provide an overview of the many practices for Healthline, Jan. 2019. 
planted in their hand. These are all types of biohacking, a broad term for a lifestyle that's growing increasingly popular, and not just in Silicon Valley, where it really took off. (Samuel, "How Biohackers")

In Samuel's enumeration we see how 'biohacking' or 'do-it-yourself biology' has become a cultural phenomenon - the media report on it, TV shows use it, "cultural heroes" and celebrities endorse it. This prevalence makes it an "increasingly popular lifestyle" (Samuel, "A celebrity biohacker"). Most people, as Samuel writes, have come into contact, if not with the term, then with one of the many biohacking practices out there. Most likely, in fact, we have all engaged in some form of biohacking, for example by changing our diet or lifestyle, taking up yoga or meditation practices or taking supplements to increase our feeling of health and wellbeing. ${ }^{7}$ This ubiquity, however, has made it notoriously difficult to define what counts as DIY practices, to delineate their boundaries, characterize their actors and expose their politics.

\section{The Biological Maker}

Since the turn of the century, the media hype around molecular and synthetic biology, most popularly the Human Genome Project (HGP) and advances in genetic engineering, has led to the spread of biomedical knowledge out of the domain of expertise and into the public sphere. This popularization increased the knowledge of lay populations and with it their eagerness to participate in the dialogue and decision-making processes. People became not only actively engaged in the discussions around biotechnology, but also in the practice itself. Starting in the mid-2000s, we can witness an increasing rise in commercial and private initiatives that aim to make knowledge about oneself or the biological world more readily available: do-it-yourself (DIY) Biology and Medicine. Taking inspiration from the do-it-yourself ethos in American culture and its latest rendition in the maker movement, community workspaces and laboratories have started to pop up around the US (and world). These spaces allow experts and interested lay people to experiment with biological materials. Such experiments can range from creating new and cheaper tools and technologies, to hacking yoghurt, creating fluorescent organisms, or sequencing one's own DNA. Similar tendencies can be observed in commercial or mainstream (medical) markets: direct-to-consumer genetic testing, medical home health tests, body hacking and self-tracking all emphasize a self-reliant, proactive and curious patient-consumer.

Positioned on the crossroads between biology, medicine and culture, these DIY initiatives are the subject of this book, both as practices and through their cultural representations. The diversity of practices that are considered to be forms of biohacking or DIY biology, however, has made it hard to pinpoint their exact meaning - especially

7 By using 'we' in this book, I do not seek to speak for all Americans, which I as a Cerman national, residing in Cermany, of course cannot. I do, however, want to point out that, even though my analysis is located in Cerman American Studies, the DIY trend in medicine and biology is not an exclusively American phenomenon. The 'we,' therefore, is an attempt to signal community and continuity, to signal that some of the concerns I address can also be translated into a global perspective. 
because both terms are often used interchangeably in cultural representations and publicly available knowledge. Likewise, academic inquiries into biohacking do seldomly end with a concise and clear-cut definition of what these practices are. Rather, they tend to focus on their ideological origins and underlying values, among them knowledge sharing, a community orientation, rebellion, access, participation and openness (Delfanti, Biohackers; Keulartz and van den Belt; Kelty); the spaces and places of its practice (Meyer, Domesticating); their experimental approach (Delgado and Callen; Delgado) or their relations to other, similar movements or social and economic institutions (Delfanti, "DoIt-Yourself"; Keulartz and van den Belt; Aguiton and Tocchetti).

To reflect the diversity of practices, I have chosen to employ a broad definition: I use the rather long phrase 'DIY biology and medicine' as an umbrella term that includes the 'hacking' and tinkering with biology, the experimental, activist engagement with biology, as well as more mundane and commercialized alternatives. As these wideranging practices already show, one of my hypotheses is that we should view them as intrinsically connected, as going back to the same ideas and basic propositions. While some DIY techniques are concerned with biology in the wider sense, others deal with the human body more closely. What connects them are a focus on openness and curiosity, education and (scientific) literacy, self-direction and competence, as well as a view of biological life as plastic. They all require some element of personal agency and autonomous decision making on the part of the patient-consumer. This self-directed nature draws on some of the core values of American culture: individualism, autonomy, choice. In a culture that sees individuals as responsible for their own situation in life and values self-reliance and initiative, the figure of the maker, the active shaper of their fate, serves as a cultural ideal. It is this self-directedness, as I will argue, that makes this case special: DIY, while seemingly giving authority to people, in truth subjects them to another, new ideology that is so persistent because it relies on basic American cultural values, heightened to an extreme.

These 'DIY techniques' are at one and the same time cultural, social, political and material: They regulate human action and behavior, shape the construction of (new) subjectivities, create new forms of authority and agency, give rise to new methods of social control, and last but not least shape contemporary body concepts and material bodies - they directly impact on (human) biology and leave their traces on the body. By doing so they take part in several ideological projects: knowledge transfer, patient empowerment, medicalization of society, government of bodies and communities, enhancement, and consumerism, all of which will play a role at some point during the course of this book. The reach of these DIY biology and medicine projects, however, is still hard to gauge, if we follow Morgan Meyer, because as an emerging phenomenon it takes place in more shielded spaces (Domesticating 7). But DIY biology is not just private - less visible in its domesticated, un-institutional spaces - but also highly public - visible in the media, through outreach activities, and the open philosophy of its community spaces (Meyer, Domesticating 7). It is this public, visible side that receives most of my attention. 


\section{Somatic Society and the Molecular Body-Project}

As the examples in this introduction have shown, biohacking is in the "zeitgeist" (Hins): Not only is it an accepted and expected lifestyle and consumer fashion (Hins' more colloquial use of the term), but more abstractly it is an expression of its time and place that is very much situated in the American cultural mentality and political climate. As such, it is no wonder that biohacking is shaping and shaped by some of the dominating cultural phenomena, debates and problems of today, some of which I want to briefly point out in this introduction.

Underlying my examination is a distinct understanding of how knowledge 'travels' in the public sphere: Originating in the lab (to follow Latour and Woolgar), it arrives in popular discourse where it is inserted into culture (with its narratives, images, symbols, and so forth) and ideology, which then influence the behavior of people individually and collectively. ${ }^{8}$ The desire to participate in biological practices and decision making is merged with a general trend towards DIY already present in popular culture. Following this observation, DIY biology and medicine can be read as being dependent on existing cultural narratives of (biotechnological) progress as well as ideologies of perfection, which in turn feed into and are sustained by some of America's core values. Cultural practices and cultural representations, in literary or more broadly 'textual' form, then perpetuate biohacking's ideology and promise, thereby contributing to the formation of a (new) politics of life, characterized by self-directed interventions into the body in order to evaluate and enhance its performance.

Making the already good 'better than perfect' is deeply embedded in American culture and has been made easier in the past centuries through the rapid advancements in medicine and biotechnology - now going beyond transformations through hard work, surgical intervention or changes of the body surface, towards optimizations on a molecular scale. The transformations in biotechnology during the second half of the 20 th and the first decades of the 21st century have created new techniques of "penetrating, viewing, and controlling bodies" and by doing so "exponentially intensified debates about what it means to be human" and to have a body (Wegenstein, Getting 87). The body today is no longer seen in its totality, but has become fragmented and molecularized, imagined as a collection of parts that can be repaired, replaced and improved. The most meaningful parts of biology today are not tangible, or even visible to the naked eye. Rather, they are hidden deep inside the cells of the body. To reach them, we need to "get under the skin" (Wegenstein, Getting 79). Molecular biology, genetics and biochemistry culturalized the body in new ways (Braun 7) and changed the ways in which we make sense of it, on an increasingly molecular scale. ${ }^{9}$

8 cf. for example, Dorothy Nelkin's Selling Science (1995), Sharon Friedman's Communicating Uncertainty (1999), or Mcinerney et al.

9 Biotechnology in one way or the other always depends on the wish to understand and control processes on the molecular level. This desire for conscious molecular control and manipulation encourages scientists to find ever newer techniques and tools to "read, edit, and rewrite the molecular mechanisms of biology" - DNA, RNA, proteins, immunoglobins, lipids, polysaccharides, vitamins, hormones, neurotransmitters, metabolites, all the small molecules that play a role in biological processes (Stevens 16-19). See also Ch. 3.1. 
This molecularization has miniaturized the targets for biological control and transformation: It is no longer only the surface of the body that has to be known and shaped but also its molecular make-up, which gives rise to (DIY) technologies aiming to establish a (molecular) status quo. While for a long time, the body was constructed as a biologically given, more recently it came to be seen as both culturally constructed as giving and making meaning through its performance - but also as a possibility, a malleable entity that can be shaped to fit the desired performance. People can and even are expected to shape their bodies inside-out according to their liking, reaching from weight and shape, to cosmetic products and elective surgeries, to modifications and tattoos. The body becomes a medium for the expression of the self (Rose, Politics of Life 8). Human life, human embodiment becomes not a mere given, but a project - a process of enhancement, modification, tinkering, active intervention - that is realized not just in biology but also in society and culture. ${ }^{10}$

We live in a "somatic society" (Turner) in which the self and the body are governed in new ways (Wegenstein, Getting 4). The ideology of healthism and the ritual of self-surveillance are meticulously observed in this body-centered society (Chrysanthou 472): People try to make their bodies transparent, to access their interior landscape through (self) screening and diagnosis, to conquer uncertainty and contingency through a healthy lifestyle and 'responsible' choices, subjecting the body to a new, all-encompassing clinical gaze - their own. When consumerism and market ideologies dominate the health care system, health is commercialized, knowledge becomes a commodity and the patient a consumer. Active participation in health care is regarded as a cultural imperative for (neoliberal) subjects, shifting responsibility and blame for their physical condition to the patient, for example when they do not take up this role. In this setting, knowledge of the self, of the condition of the body and state of health becomes the foundation for informed, responsible decisions. This knowledge is no longer only generated in cooperation between health care professionals and patients, but also by patients themselves. Shifting power relations and the trend towards patient empowerment and autonomy have turned them into the ultimate authority over their bodies and health interventions. In his study of biopower, biopolitics and the medicalization of human life in the 21st century, The Politics of Life Itself (2007), Nikolas Rose argues that amongst others the molecularization of life and the strive for optimization have transformed the Foucauldian notion of "biopolitics" in the 21st century. In consequence human life processes are today not only subordinated under government control ("bio-power") but are inextricably intertwined with identity politics and (bio-)economics. In this new "politics of life itself" techniques of self-control and management of the body - in line with neoliberal bio- and body politics - become increasingly important, power relations are consolidated (as in the role of expertise) and reshuffled (as in the increasing agency and autonomy of patients), new forms of life (the person at risk, the susceptible self) and new forms of community (biosociality) emerge, and molecular rationales determine the governance of bodies, selves, and society (Rose, Politics of Life). 


\section{Promise and Plasticity}

What these considerations go back to is a deep-seated believe in the malleability of biological particles and life itself, as well as a growing desire to control these processes, to make use of them to shape the development of our own bodies and our biological surroundings. Hallam Stevens delineates plasticity - the ability of life to be manipulated - and promise - biotechnology as a future-oriented, promissory science connected to hype, expectations, or fears - as important themes in biotechnological research and development. These two concepts, promise and plasticity, have worked their way into my analysis in different shapes and shades because much of the discourse around DIY biology and medicine revolves around their duality. Promises are a central feature of the cultural engagement with biology: how bio-medical-technologies are talked about, how they guide individual and collective actions, how they are used is shaped by promissory narratives in the media, in texts, films and advertisements. These promises give hope, create desires, a yearning for change, development, transformation - but they also have their dark sides: Promises can be broken, they can be misleading, they can be false. As such, promises are part of the affective structure behind biology in general and its self-made companion more specifically. ${ }^{11}$ These promises can define our understanding of our own life, our bodies and biology. They are both an opportunity and a form of oppression.

Plasticity, on the other hand, is a rather new concept that, after originating in biological research on cells and brain development, is now taken up more and more often also in cultural inquiries - or rather, that is named explicitly, when previously it had been present unannounced. Plasticity cannot just be used to describe the cultural condition of our time (requiring constant openness to change and flexibility, cf. Malabou) but also the very much material precondition for DIY biology and medicine: It requires us to see life and the body - from individual cells to the whole complex system - as a malleable entity in and of itself, as always able to change, as constantly adapting to new environments and requirements (changing both to stimuli from the outside but also on its own accord). The duality of promise and plasticity, thus, combines both nature and culture, politics and materiality.

There are transhumanistic (with a lower-case $t$ ) undertones, in the way biotechnology here seems to shape culture, creating a new cultural (and possibly in the future also human) condition (Tirosh-Samuelson). These new biological possibilities destabilize the given, the biological substance of the body - what humans for centuries have taken for granted, had to work in tandem with to achieve their goals - and create new ways of living and being in the world. A somewhat overused phrase from Marx and Engels comes to mind: "All that is solid melts into air." Famously utilized by Marshall Berman to describe the experience of modernity with its constant fluctuation between creation and destruction, unity and disunity, disintegration and renewal, it lends itself to capture some of the uncertainty but also creative potential that comes with biotechnological and

11 Fear and anxiety can be considered as the 'negative' counterparts to hope and promise. They similarly guide our understanding of and responses to biotechnological innovations or biological threats. 
medical advancements. The body, its mental capacities and physiological condition, are no longer a given or limit. Instead, in these new "regimes of living" (Collier and Lakoff 23,34 ) individuals through self-surveillance, self-management, and (self-)enhancement turn their bodies into possibilities. This certainly is a future-oriented process, driven by the wish to conquer and control the last great unknown ("What will happen to me in the future?", "Will I get sick?", "What will my children be like?") and replacing it instead with an ostensible certainty, centering around what the body can do, how it will look like. This certainty is only spectral. There are so many contingencies in human life, that they are impossible to control (at least in free societies, dystopian versions can be found aplenty in fiction). But DIY also carries with it transformative potential for processes of knowledge production not just of its application to human bodies. The success of DIY can also lead to an epistemological shift, in which hacking and tinkering - as epistemological practices - are blueprints for a new form of creating knowledge and a rebellion against outdated systems of power, differential ascriptions of competence, and exclusive restrictions on access to technologies, goods and knowledge.

An analysis as widespread as is attempted in this book shows not only the cultural and material effects of recent biotechnologies but also the high personal, social, and political stakes of these technologies. 'Do-it-yourself' creates a new cultural politics of life centered around self-reliant individuals as both decider and executer, diagnostician and practitioner, subject and object, observer and observed, taking their health, their lives, their biology literally into their own hands. The questions guiding this examination mirror the multi-leveled nature of the project: What are the social, cultural, and indeed material effects of DIY as field of practice, a "technique of the self" (Foucault)? What is its reality as embodied phenomenon; as object of desire and hope; as form of governance; as generator of capital? What is its culture, what are its politics? How can DIY biology as part of the promissory economy of biotechnology effect transformations in how we produce knowledge, approach bodies and indeed 'life itself'? How does it shape the understanding of the self and biology? What kinds of conflicts are embedded in DIY biology? What are the ways, means, and forms in which DIY biology and medicine are inserted into culture? What is the potential of this cultural trend, what are the negative side effects and how are they discussed in the public sphere? What narratives, metaphors, images are used in these discussions? These are only some of the questions that will be addressed in the following chapters.

\section{Representations of Biohacking}

The terrain covered in this book is a field of study that has only reached the disciplines of Cultural Studies and American Studies in the past decades, fueled especially by advancements in biotechnology and possibilities of medical intervention that caused major turning points in fundamentally cultural and social questions, such as identity, heredity, race, gender and so forth. With some of these up for re-negotiation, cultural theorists have taken up the challenge to provide criticism of regimes of knowledge production, laboratory practices, or the ways and means through which scientific information and concepts are carried into and debated in the public sphere. Such approaches, 
what Lennard Davis and David Morris in 2007 have termed "biocultures," instead of limiting their scope to either side of the 'biology vs. culture'/'natural sciences vs. humanities' debate, argue for a continued dialogue between these fields of specialization. As Davis and Morris maintain, looking at "the biological without the cultural, or the cultural without the biological, is doomed to be reductionist at best and inaccurate at worst" and therefore we need to re-think culture with its relation to biology in mind and vice versa (411). ${ }^{12}$ In my arguments, I focus on the culture of DIY biology, or rather, how culture intersects with DIY biology.

I aim to explore how those techniques are represented and negotiated in the public sphere, to consider the politics of DIY through their representations in order to expose the connections between the material practices and their social, cultural, and political dimensions. Situating the emerging discourse of DIY biology and medicine in a historical, political and cultural context, allows me to examine the underlying beliefs, discourses, and ideologies behind the current turn to DIY technologies as well as the new imperatives, narratives and politics created by them. This focus on representation can expose the biological and cultural imaginaries (cf. Steinberg) created by DIY biology and medicine; show how people debate, criticize, re-imagine interventions into the human body; elucidate the fears, longings, fantasies, desires connected to such technologies; and investigate how personal and political meanings are created about them and how they subvert and reinforce dominant ideologies.

Essential, here, is the cultural imaginary of biology, the "dominant cultural metaphors, symbols, images, and representations" of a given culture (Mackenzie 125), in this case in their relation to biology. With broad reference to Winfried Fluck, culture is understood as a medium for the (fictive) expression of such imaginaries, through which we can supersede our own lived reality and imaginative horizon, on a trial basis (12-13). Through imaginary representations, we can 'try on' different identities, different types of embodiment, different social and political systems in fiction - give expression to thoughts, feelings, experiences and fantasies without fearing immediate consequences (cf. Fluck 15). ${ }^{13}$ Cultural representations show how meaning is attached to practices,

12 While Davis and Morris' seminal argument about the need for "biocultural" scholarship has been formulated more than a decade ago - and could thus be considered outdated - younger scholarship still addresses the continued relevance and need for such engagements, cf. Pitts-Taylor, "Mattering" (2016).

13 The cultural imaginary can also impact our experience of embodiment. Here, the notion of a cultural imaginary acquires a meaning apart from its descriptive use as an umbrella term for all sorts of cultural productions. Especially the latter part of the term takes on a more literal meaning. Catriona Mackenzie argues that norms and cultural fantasies are enforced and perpetuated through metaphors, symbols, and visual representations (125). But how do these cultural metaphors, symbols, images and representations affect the experiences and self-concepts of individuals? The suggestion Mackenzie develops is that the availability of images and representations can constrain our imaginative possibilities: our ability to imagine ourselves (otherwise) draws on the cultural repertoire of images and representations $(126,143)$. The available images and representations determine what imaginative possibilities we see for ourselves and narrows our options for imaginative explorations of alternatives. The cultural imaginary, thus, also influences how we can see ourselves and construct our identities - what we canimagine ourselves to be. This can affect how we view and experience our bodies, in sickness and in health. 
bodies, and forms of life: What is represented can be imagined, what is imagined can find expression in representation (without the consequences of the real). Representations of DIY do not only shape how the public (and scientific-medical community) sees and evaluates these techniques, but are also the results of the shared meanings, desires and values of a society, which highlights the dynamic relationship between representations, technologies and the public. But representations can also, and crucially in relation medicine and the human body, have material impact. In her study of Andean imagery, Deborah Poole introduced the term "image world." She employs this concept to describe the material and social nature of images, arguing that how we see and represent the world decides "how we act upon that world and, in so doing, create what that world is." Seeing and representing, thus, are "material" because they create ways of intervening in the world (7).

Some sociological, ethnographic and natural-sciences work has already been done on DIY biology, biohacking, the Quantified Self, and genetic testing. ${ }^{14}$ Topics in this research include biosecurity, its political nature and mode of activism, its tools and spaces, the relationship between experts and amateurs, its norms and ethics. But so far not so much work has been done on DIY biology and medicine's cultural manifestations and representations. But culture (art, literature, performance, or popular) and its academic analysis can make available new perspectives not just on topics dealt with in the work of 'art' but also on 'scientific research' in general. As Stefan Beck claims, science and humanities need to work together to coproduce new conceptual instruments, a new language and vocabulary, to facilitate social and cultural engagement with and understanding of biomedical "in(ter)ventions" as well as reflexivity about the process of knowledge production (18). Literary and cultural studies can contribute a great deal also in the field of (natural) science: "the influence of language, narrative, and images on public attitudes toward science," means that "scholars who are experts in interpreting cultural matters" are uniquely equipped to take part in the discussions around the development and use of scientific knowledge and technologies (Wald and Clayton x).

More than that, such scholarship can make available different, distinct forms of knowledge. The importance of such research, therefore, also derives from the fact that humanities enquiries come from a different disciplinary background, have different philosophies of life and different approaches to how scientific research should be conducted: the same questions can lead to new insights when research is framed in another way or distinct categories are applied; new ways of collecting, organizing, processing and interpreting "data" can lead to unforeseen hypotheses and conclusions that are then transported to the public in a new way (Wald and Clayton $\mathrm{x}$ ). Most of the time, what is needed is also a broader understanding of 'data' that moves beyond 'hard facts' and acknowledges that texts, narratives, images, metaphors and so forth can also be used to draw conclusions about the meanings created in specific social formations. ${ }^{15}$

14 cf. among others N. Fox; Roosth; Meyer ("Build"); Meyer ("Hacking”); Delgado; Delfanti (Biohackers); Delfanti (“Users and Peers"); Lupton (Quantified Self).

15 The task of cultural critique, however, is not primarily to assess the viability, even desirability or morality, of these current trends. While ethics will, to a certain degree, always be present in this 
The sources used in the following analysis reflect exactly this approach to 'data.' The new bio-medical and -technological trends and possibilities are negotiated in public culture by utilizing materials, scripts, narratives, imaginaries already available in the cultural archives but also by creating new ones. They give representation to how the possibilities of biotechnology are experienced, interpreted, and made public - how they make meaning in the public manifold. Cultural artefacts become favorite sites for the negotiation and (re)production of meaning; and this meaning is debated in diverse sites ranging from practices - in laboratories, court rooms, hospitals, and domestic spaces to texts - in mass media, textbooks, legal cases or science fiction. Using interpretative research methods and textual analysis, I want to explore the rise of the DIY movement and its ubiquity by touring its sites and texts. As Lawrence Grossberg writes, the starting point of cultural studies is always an assemblage, never a single event, text, genre or medium (223). Looking for shared discourses, repetitions of themes and conceptualizations I will use the 'texts' under consideration as entry points into their context and background, to connect them to broader social, economic, political, cultural situations, to map their configurations and contradictions. I will therefore analyze the examples not as stand-alone practices but in their assemblages, situate them in a wider network of practices, relations and affects.

Given the impact of mainstream and popular treatments of these topics on public perceptions (Wald and Clayton xi), these 'texts' are not restricted to complex and ambitious literary works, images, and cultural productions, but instead primarily encompasses those considered to be pop-cultural. These include 'subcultural' texts like magazines, websites, or individual and collective self-definitions by the DIY community but also their perception in mainstream culture. What is crucial, furthermore, is that the sources are not limited to a particular domain, such as only visual or only textual. Instead, they encompass all the resources used to make sense of the changing relationships between bodies and technologies: (social) media, journalism, popular science writing, images, films, narratives, commercials, (science) fiction. ${ }^{16}$ This broad range is chosen consciously in order to engage different configurations in which biological knowledge is turned into cultural practices but also to account for the higher proliferation of DIY in pop-cultural formats due to their reliance on contemporary, social media. The diversity of practices and cultural representations, I would argue, is not so much a problem but rather a strength because it demonstrates how wide-spread DIY logics are employed in biology and medicine. It is not a singular phenomenon but one that is pretty much ubiquitous and spreads into all sorts of everyday (cultural) practices. At the same time, the diversity allows me to distill the common concerns and problematizations, to examine the underlying values and future potential. All the objects and practices studied here reflect central debates that are (or need to be) held about the changing configurations of technology, biology and human agency.

This project has "reshaped" itself as I went along, included new texts and contexts, new problems and conjunctures (cf. Grossberg 1). But as with any academic analysis, at

examination, I want to move beyond addressing questions of desirability or morality and move to more complex questions and fundamental issues. 
some point I had to draw a line. For me, this line was somewhere in early to mid-2019. I have included newer developments in footnotes where appropriate, but as a field that is still in constant flux some of them might not be addressed. Moreover, that biotechnology changes at an unprecedented pace - and with it the possibilities of self-intervention - might mean that such a study could become outdated once it is finished. This is a valid concern that needs to be addressed. It might be true that some of the techniques under examination here might get 'old,' both in the sense of becoming routinized and in the sense of getting outdated by newer technologies. It is, however, not so much the individual techniques that count but the values and ideologies that underlie them, the visions of the future created by them and their (fictive) narrativization in culture. These basics will remain relevant even if the perspective on and evaluation of DIY techniques changes.

\section{Little Strokes and Big Oaks}

Our understanding of life - both its biology and embodied practice - is continuously transformed by the introduction of new concepts and ideas, patterns of knowledge and ways of thinking. Changing the prevalent "ways of thinking" has transformative potential. With a new approach and perspective, what seemed unthinkable suddenly becomes possible, desirable, even necessary, so that epistemological shifts can lead to cultural shifts. Transformations during the $21^{\text {st }}$ century have, according to Rose already led to new configurations of knowledge, authority, technology and subjectivity (Politics of Life, 104). These new configurations both serve as a basis for DIY techniques and are continuously transformed by them.

I am here following Rose's line of thought in suggesting that all these shifts are not epochal, drastic changes, but incremental developments. The changes brought about by biotechnology are not a rash rupture or sudden change, but a slow and steady move a process of ever new inventions, a process of normalization of what was thought to be unthinkable and undoable (Rose, Politics of Life 5; 252). As Karen-Sue Taussig claims, transformations do not "just happen", "[n]ew ways of thinking about the world do not simply seep into the collective unconscious" and "new social and embodied practices [do not] emerge from nowhere;" rather, transformations only have an effect when they "have some basis in material reality" and are "taught, learned, and experienced" (193). In The Politics of Life Itself, Rose argues that it is important to show that we live in the middle of "multiple histories," that many futures are still possible. It is not a singular narrative from here to the "posthuman future" - that some desire and some dread - but a complex web of contingent pathways, which together will create something new (5). Once they are in widespread use, even daringly radical innovations will become routinized and appear normal, the changes will be "gradual rather than revolutionary, incremental rather than epochal" (Politics of Life 5, 252). The gist of what Rose is arguing here is that we always need to keep in mind that also the accumulation of small, seemingly not so drastic or even disparate changes can create something unprecedentedly new; even apparently small changes in the ways we think and perceive the world can open the door 
for more radical ways of configuring life and should thus be examined critically before they become routinized.

Because these are slow, repetitive processes, it is always useful to look at the past, to analyze the harbingers of future developments, in this case, older strategies of selfmanaged, self-directed intervention and optimization. Contemporary transformations have not come about in a vacuum, but rather have been prepared by and depend on prior processes; they have a long history that they cannot be understood without. That there is nothing really new or fundamentally different about these developments - apart, of course, from the techniques employed and their far-reaching consequences - can be shown by pointing to historical examples, precursors, and the underlying values, desires and debates (Rose, Politics of Life). Looking at the historical continuity of recent phenomena tells us that they are here to stay, that they are simply old and deep-seated desires and fears played out on new playing fields. These desires and fears shape how both the practices and their representations are negotiated in the public manifold. Culture and society - in all their differing shades, forms, and shapes - play a crucial role for the perception of science, technology and medicine in society and thus become increasingly important for a full discussion of contemporary and future uses of biotechnology, for an assessment of personal and communal responses to and valuations of them, for meaningful debates about how (or whether) to regulate them.

\section{What to Expect}

To account for this complexity, the argumentation in this book will need to address multiple levels, reflected in the overall outline of the project. Chapter 2 "Biology and Culture" provides an overview of the directions that humanities enquiry into topics broadly understood as 'biological' has taken but also an introduction of the most important concepts needed for my analysis, such as the constructed and communicative nature of biology; the importance of bodies and materialism, affect theory, promise, and plasticity; discourses of governmentality, responsibility and citizenship. Part II Background is split into two chapters: Chapter 3 "The Promise of Small Things" takes up the idea of molecular biology as cultural and engages with biology as a (pop)cultural phenomenon. It illustrates how the cultural presence of molecular biology and biotechnologies is also a central component of DIY biology because it resources (utopian and dystopian) fantasies of perfected bodies and creates pervasive discourses that influence how humans think about their biology, transforming it from a certainty into a possibility and (life) project. To do so, I follow a trajectory from the molecularization of biology and life, via the signifying qualities of molecules - primarily as promises of enhancement and symbols of vulnerability - towards the fantasy of perfected human bodies. Richard Powers' novel Generosity: An Enhancement and the imaginary engagement with SuperHumans provide narrative and cultural examples.

On this basis, Chapter 4 "With My Own Two Hands" grounds DIY biology and medicine in American cultural values and ideologies as well as material realities. It traces the rise of 'do-it-yourself' as a cultural practice and imperative, looking both at its historical development and transformative potential. I discuss some of the founda- 
tional (American) values that influence DIY until today, including but not restricted to individualism, a belief in progress, enhancement, and perfection (Chapter 4.1). A historical trajectory of DIY from its early days of home improvements to its newest renditions in the maker movement and citizen science (Chapter 4.2) as well as a discussion of the DIY patient in the context of medicine (Chapter 4.3) demonstrate that the 'newer' examples are not fundamentally new but grounded in long traditions. DIY biology and medicine are, essentially, old practices in a new guise, continuations of foundational American myths of innovation, entrepreneurship and self-reliance. New technologies, new possibilities, new visibility gives them a new quality and have turned "life itself" into a hackable entity.

Part III, 'Hacking' Life Itself, brings the previous chapters together by showing, through readings of DIY texts and practices, how life is 'hacked' in contemporary times. Drawing on academic and pop-cultural sources, Chapter 5 attempts to find a definition, or at least a common understanding, for the terms and practices I consider under the umbrella term 'DIY medicine and biology.' The following five chapters, then, introduce many different DIY techniques both as practices and through their cultural representations. Primary texts for analysis are representations of DIY biology that circulate in culture, including popular non-fiction science writing (such as Marcus Wohlsen's Biopunk), instructions and guidebooks (the 'self-help' sector, such as James Lee's Biohacking Manifesto), corporate public relations material and self-descriptions, as well as media coverage. In each chapter, introductions to the chapter topic and main themes are followed by two case studies that shine a different, sometimes contradictory, light on DIY practices in that respective sphere: Chapter 6 "\#iambulletproof" looks at biohacking as a form of self-optimization. Based on print and online examples, it demonstrates how these self-help resources further a rhetoric of responsibilization, in which (following the logics of neoliberal capitalism) the individual is responsible for their own health and wellbeing. In the guise of translators or mediators of knowledge, self-help gurus rise to a new status as "experts of the soma" (Rose, Politics of Life 5-7).

Chapter 7 "A Pill for Every Ill" contrasts the rising stimulant 'epidemic' of ADHD drugs and nootropics on campuses and in the workforce with a consideration of DIY medications and drug hacks for cheaper, universal access to lifesaving medications. In a culture focused on constant competition, availability and performance, stimulants are no longer only used to treat medical conditions but also for DIY projects of optimization. Drug hacks like the "DIY EpiPencil" from the Four Thieves Vinegar Collective, on the other hand, promise to provide access to medications to those who cannot afford them in a commercialized healthcare system faced with rising prices and low solidarity. In a similar fashion, Chapter 8 "Test Yourself" looks at commercially available tests and their opposite: creative, subversive and personal 'hacks.' DTC gene tests and molecular health tests by commercial providers are marketed as tools of self-knowledge - promising individuality, opportunities for optimization and a sense of community. Kay Aull's self-made DNA test has become famous in the DIY scene and has been a focal point of non-fiction reporting on biohacking as well: Even though this is an emancipatory act, Marcus Wohlsen's portrayal in his non-fiction book Biopunk embeds it into a similar discourse of self-knowledge and self-enhancement. 
Chapter 9 "Homo Technologicus?" traces the changes technology has brought to ageold practices of self-reflection and introspection: It contrasts those technologies tracking from the outside (e.g., the Quantified Self movement) with endeavors that seek to embed technology into the human body to create DIY cyborgs (grinding). These practices have immersed themselves in daily life also through media reports, cultural artefacts and transhumanist thinking. Chapter 10, "The Human Need to Fiddle", highlights practices in the realm of DIY biology and medicine that proceed open-ended, communityoriented and participatory and thus might have the biggest potential for true democratization. On the one side, it looks at spaces, tools and technologies that have been created in the DIY biology scene (through the lens of self-descriptions and Wohlsen's framing in Biopunk). Those tools aim to provide access for all who are interested, reduce barriers of access and offer cheap materials for (more or less playful) experiments with biological materials. On the other side, it closely analyzes the discourses created around open-source, DIY technologies already in use in a medical setting: DIY continuous glucose measurement devices (here, the Nightscout project) and open artificial pancreas projects. In these cases, self-made technologies are used to monitor and even modulate the biological functioning of the human body.

Part IV, Reflections, then untangles what the consequences of the many different but related practices and representations of DIY biology and medicine are (or might be). The discussions in this final part reflect on questions of access, participation and democracy (11.1); the effects of consumerization and security concerns (11.2); discourses of empowerment and their counterpart, heightened vulnerability and precarity (11.3); and the risks of social stratification and the nascent sense of collectivity (11.4). In each of these subchapters, overarching themes from the previous analyses are discussed, abstracted and brought into dialogue in order to carefully deliberate positive and negative aspects of this not-yet-fully-formed trend. After having reconstructed the discursive formations and contexts in the previous chapters, I identify sites of struggle and potential, the promise and politics of 'DIY biology and medicine.' 


\title{
2 Biology and Culture - an Overview of the Field
}

\author{
"Biology, as a science, cannot exist outside \\ culture; culture, as a practice, cannot exist \\ outside biology." (Davis and Morris 418)
}

Biology and culture, science and humanities - disciplines that traditionally have been thought to have nothing to say to each other, that are believed to have widely different epistemological approaches, have in the last three decades been brought into dialogue to tackle some of the more fundamental questions of today. The contested issues of the $21^{\text {st }}$ century never only concern nature or culture, they are always both natural and cultural, social, political. We can no longer think of them as separate entities: Biology, as Davis and Morris argue in the introductory quote from 2007, even as "pure" science is always cultural, and culture depends on biology. Social concepts and assumptions drive biology, culture cannot take place outside of the lived, material reality of everyday life (418). This becomes nowhere more apparent than in the many issues surrounding biotechnology. The recent headlines about scientists being able to "delete" or rather cut out genetic defects using CRISPR technology, "genetic scissors," in embryos during invitro fertilization are a prominent example, such as the highly contested germ-line engineering performed by a Chinese scientist in 2018 (Kolata et al.). ${ }^{1}$ The ensuing questions of treatments versus enhancements, justified and unjustified use, access and affordability, social justice, civil rights and discrimination, value judgements and normalization already point to the multiple convergences of biotechnology - as applied science - and

Dr. He Jianku used CRISPR/Cas-9 technology to alter the genome of twins to be resistant to HIV by deleting a gene sequence responsible for creating a protein that the H.I. virus needs to enter cells. His experiments have received wide-spread critical backlash in the scientific community and society around the globe because they mark the first publicly known attempt at modifying the human germ line. Subsequently, his research activities were suspended, the Chinese government as well as his university investigated his work (leading to the termination of his contract with the university) and he may face more consequences. In March 2019 it was reported that the mutations $\mathrm{He}$ Jianku provoked may (inadvertently) have had an effect on the babies' cognitive abilities - raising the possibility of cognitive enhancements (Regalado, "China's CRISPR"). All in all, the controversy around $\mathrm{He}$ Jianku's experiments is also part of an on-going race in biotechnology between China and the USA in particular, with China often described as enabling more controversial research to win this race. 
culture. Investigating biology from a cultural standpoint, then, can shine light on both the effects and the epistemic assumptions behind biotechnology's current appeal.

As Davis and Morris claim: The "specter of biocultures is upon us." They had a "new (but perhaps in a while old) and counterintuitive (but perhaps destined to be commonplace) proposal: that culture and history must be rethought with an understanding of their inextricable, if highly variable, relation to biology" (411). In the past decade since this statement has been formulated, doing "biocultures" has become more and more common but not yet commonplace. Davis and Morris' assumption, however, remains true more than ever. To fully grasp the scale and future effects of biotechnologies' sway over contemporary daily life, science needs to be thought of also from a historical and cultural perspective, and literature and culture need to be engaged from a scientific one (411). Likewise, Victoria Pitts-Taylor, in 2016, argues that transformations in the sciences have promoted the naturalization of other fields of knowledge: the dualism between social and biological - as well as the respective disciplines - is "being supplanted by the more monist notion of the "biocultural"' (1). The omnipresence and amalgamation of biology and culture expressed in the term biocultural in contemporary life makes it necessary to not only address one or the other but both (Pitts-Taylor, "Mattering" 1 ). They need to enter into an even more sustained dialogue, one that is mutually beneficial by strengthening science and deepening cultural analysis. This is even more true in 2021, Shiloh Krupar and Nadine Ehlers claim: "the time for biocultural analysis is now" (14, italics in original). Following Davis and Morris' approach, for them "the body and culture are never separate" and "biomedical and cultural arenas are enmeshed" (9). ${ }^{2}$ They argue that biocultural studies are a tool to understand how biomedicine today extends far beyond its original sphere of the clinic, lab and hospital into social practices, biosecurity concerns and biopolitical governance. ${ }^{3}$ Key to the study of biocultures, for them, is not only a biopolitical focus but also a certain methodology that is "intertextual, collaborative, [and] multiply-scaled." "Intertextual reading[s] between various kinds of cultural products, knowledges, policies, and practices" are combined with cross-disciplinary blends of methodologies and approaches - bridging different "regimes of knowledge" (Krupar and Ehlers 7-9).

The human body is also at the center of Samantha Frost's use of the term "biocultural" in 2016. Frost rethinks humans as "biocultural creatures," as made in and through convergences of the biological and cultural in the human body itself. Humans are not self-contained entities but interact with "substances, creatures, and organisms" (Frost 148), even on the molecular and atomic level. Human bodies, human materiality, are cultured within their material, social and symbolic world.

3 In light of the Covid-19 pandemic and its biopolitical effects, Krupar and Ehlers' reading is very much focused on the level of biopolitical governance and biopolitics. They look at "health issue terrains" as the arena and sphere of biocultures, in that here "biomedical logics extend beyond the confines of medicine to govern populations and discipline individuals" (5). They advocate for biocultural analyses that move from micro scales of individual bodies to macro levels of institutions and population-level governance - "from the institutional to the individual to the molecular" (10) - but they also consider how "life-making practices" can create deadly conditions and "perpetuate inequitable distributions of life" (11-12). Especially the later chapters (11 and 12) of this book look at different levels of biopolitical governance as well as the negative side effects - discrimination, unequal access, vulnerability - that come with DIY biology as a biocultural practice. 
As a methodology biocultural studies, as Krupar and Ehlers argue, can thus integrate insights from many different disciplines and theories. Fittingly, sources and inspiration for this book come from a variety of backgrounds: These reach from classical considerations of social constructionism in Science and Technology Studies (STS), theories of medicalization, knowledge production and dissemination - the cultural basis of science - towards body studies and the renewed focus on the material reality and basis of life (including theories on biological and cultural plasticity and affect) as well as concepts taken from theories of governmentality and (bio-)power. While it would go beyond the scope of this book to go into detail in all these fields, I would nevertheless like to acknowledge how some of them have shaped my understanding of the questions at hand: Science and Technology Studies, for example, has influenced my discussions with its focus on network-oriented thinking and the role of researchers and research settings in the production of knowledge; medical sociology has brought to the table considerations of power relations in the medical encounter and the importance of socio-cultural contexts for the construction of knowledge and the experience of disease (cf. Lupton, Medicine); from the medical humanities I have taken my focus on literature, textual study, signification, discourse analysis, narrative structures and the construction of meaning, while medical ethics, though subconsciously, resonates in questions of discrimination, access and distributive justice.

Cultural Studies proceed from a sense that no aspect of human life can be separated from the questions and effects of culture, that also other disciplines need to be partially understood through culture. This inherent interdisciplinarity - bringing together diverse disciplines, organizations of knowledge and "bodies of expertise" - offers unique possibilities for the study of biology, medicine and biotechnology because at the same time that Cultural Studies questions the cultural construction of reality it also beliefs in and acknowledges the importance of material realities (Grossberg 15). This preparatory chapter provides a (literature) overview of the directions that humanities inquiry into topics broadly understood as 'biological,' 'corporeal' and 'medical' has taken as well as an introduction to the most important concepts needed for my analysis. I emphasize these conceptual issues and methodological approaches, however, without claiming comprehensiveness or completeness. Rather, my aim is to provide a set of tools that have been developed in the study of 'bio-cultures' and which were useful for my research, to embed my analysis in existing biocultural discourses and scholarship.

\subsection{Constructing Biology}

A life without biotechnologies seems unimaginable today. Society is permeated with scientific findings and their practical applications: Genetically modified foods, genetic testing, pharmaceutical drugs, responses to global pandemics are just some of the more prominent examples. But more than that, we all "consume representations of science and incorporate them into our everyday understandings" of the world through the media, culture and education but also through scientific and medical interventions into the body (Erickson 3). Science, and by affiliation biology, we will see, is not disparate from culture, but instead incorporated into a continuous cycle of mutual influences. 
The values we assign to biotechnologies, the judgements we make about their use, the way we consume them, how, when and where we expect and allow biotechnologies to shape our lives, depends on culture. How is biological knowledge produced? How do we grasp concepts, ideas, materials that are both so intimate and abstract, highly present but invisible? How are they communicated to a broader public? What roles do language and culture play in this process? These are some of the questions addressed in this subchapter.

I here follow the position that in order to truly evaluate biological knowledge, concepts and biotechnological applications, we need to study them both as objects and as embedded and produced in a larger system of knowledge production - one that is inherently social, cultural and historical. This discussion is indebted to postmodern, social-constructivist thought and its highly influential and transformative ideas. Postmodern theories have challenged the perceived objectivity, superiority of science and the scientific method in favor of a focus on the constructed nature of scientific knowledge. Fittingly, this subchapter looks at questions of knowledge production, the role of language, narrative and discourse and the ways in which biology and biotechnology are 'culturalized' in this regard. DIY biology and medicine, in part, try to renew how and where knowledge is produced, who is involved in this production and on which terms. They want to open up a system that has been regarded as closed-off and elite for decades. For this process, it is crucial to acknowledge the cultural, social, historical influences on biological knowledge production. But the influences of culture - of narratives, discourses, the imagination - do not only shape how knowledge is produced but also how it is translated for the public and in a last step, how it affects (human) bodies.

\section{Knowledge Production}

In light of the dominance of science in contemporary life, it is no surprise that scholars have taken on the challenge to de-mystify the production of scientific knowledge, to shine a light on how the social and cultural context impact science, to question how tools, knowledges and technologies are conceptualized and brought to fruition. Science and Technology Studies (STS) have taken on this challenge. The social constructivism of the 1960 s and 70 s saw scientists as constructing not discovering knowledge, challenged the objectivity and formality of scientific practice and methods, and turned toward the places where science is "made/practiced" (such as the lab) (Erickson 86). ${ }^{4}$ According to Mark Erickson, "science and technology are social activities that reflect the social conditions of their production and the social conditions of those involved in their production" 
(1). The knowledge emerging from such scientific situations is constructed and contingent on its respective context, such as the interactions of scientists, prevalent world views, available resources. While most humanities disciplines acknowledge the high impact of social and cultural contexts on scientific knowledge production, in scientific institutions, Erickson laments, this view is not all that prevalent: rather, science is seen as a "progressive, neutral activity" producing "true knowledge and facts about the natural world" by using standard methods (2). The common understanding is that science produces more "concrete, 'better' and more factual" results (2); science is not just seen as a separate but as a superior road to new insights. This view has also made its way out of the laboratory: Society is filled with such representations of science, so that a deep-seated belief in science is persistent, shaping the understanding, expectation and in conclusion the practice of science (Erickson 2).

Science, thus, has become a dominant way of thinking, a dominant rational for how we shape our lives, as Erickson demonstrates in his thorough account of the interplay between science, culture and society. Formal science is perceived as a special way of discovering "facts," characterized by hypotheses, experiments, and the validation of hypotheses or theories based on empirical methods with their impressions of objectivity, rationality, unambiguity (Erickson 31-32). In the public's view, science is straightforward, a "progressive march towards objective truth," giving definite answers and producing "facts;" that hypotheses and theories can prove false or be revoked based on new data is often overlooked (Boyd 12). Still, this "scientific method" has given birth to one of the most prevalent, but latently overlooked -isms of our time: scientism. This standard account of science as having a higher status than other forms of knowledge production is deeply ingrained in our society (Erickson 71-75). This scientism explains why we tend to privilege scientific explanations and look to science and technology to provide solutions for many of the contemporary troubles (Erickson 23-25). That this thought style is not just prevalent in the scientific community, but in society as a whole, shapes how science and technology are perceived and evaluated in the public sphere. The cultural context becomes particularly relevant here: We have a society that on the one hand deeply, almost blindly, believes in science and on the other hand is (strictly) divided into experts and lay persons, those initiated and those outside.

However, scientism also needs to be taken with a grain of salt: Especially in the US, we can witness a declining belief in scientific findings and facts in parts of the US population. This declining trust in science is part of an overall rift in American society that has led to increasing polarization of the social and political discourse; it is part of the rhetoric of 'fake news' and conspiracy theories that have gained precedence during President Trump's four years in office. Krause et al. argue that confidence in the scientific community has been rather high - with 40 percent of Americans expressing a great deal of confidence - and rather consistent over the past decades. However, they also admit that some subgroups of the population express more confidence in science than others. According to Plohl and Musil, key variables that influence trust in science are political conservatism, religiousness/religious orthodoxy, conspiracy thinking and to some extent the level of education. So, while studies do show that overall trust in science and the scientific community is rather high and stable (with nuances), public perception of those who do not express trust in science has shifted: With the rise and popularization 
of conspiracy theories and 'alternative facts' by some of the highest-ranking officials in the government, mistrust becomes more visible and fact-checking the norm. The debate around and increasing urgency of climate change as well as the Covid-19 pandemic are two highly visible issues around which this shift in public perception and poisoning of the political discourse has revolved. ${ }^{5}$

A helpful tool in the context of scientism and knowledge production is the concept of "thought communities" - groups of people that share similar epistemological standpoints. Erickson, taking up Ludwik Flecks idea of a "thought community," defines these as groups of individuals that share a particular way of thinking, using the same ideas, concepts and theories. This "way of thinking", or thought style, is embodied in the discourse of the community. ${ }^{6}$ These esoteric - "inside" - and exoteric - wider, social - communities influence each other so that both are involved in producing scientific knowledge: The exoteric community through representation and discussion (in culture), the esoteric through research and experimentation in the lab, though influenced by cultural discourses and conceptualizations (19-23). Erickson, therefore, argues that we need to construct science and scientific knowledge production as a "two-way street between the esoteric communities of which formal scientists are members and the exoteric, public communities to which we all belong" (3). This concept makes it possible to see how what is often valued as neutral, objective, and independent is in reality shaped by processes and discourses outside of scientific practice (Erickson 19-23). These concepts also, and this will become crucial, point to the fact that science in itself is always a collective project: While individual achievements and 'genius' might be dominant scientific master narratives, the interactions between different thought communities, the entanglements with cultural assumptions, the dialogue between different stakeholders allows us to indeed see scientific knowledge as created in community.

But "thought communities" also play a role when knowledge has to travel between different groups of people. In order to bring specialized knowledge from the scientific community into the public, conscious processes of translation are needed: The concepts, terms, discourses of one thought community need to be translated into a language that is more familiar to the lay public: "In this process there is an inevitable change in meaning - we are moving from one thought community, one language-game, to

According to Boyd it is not just political factors that underly skepticism of COVID vaccines, but also a breakdown of the distinction between expert and non-expert as well as misconceptions about the "nature of the scientific process." his book Entstehung und Entwicklung einer wissenschaftlichen Tatsache; Einführung in die Lehre vom Denkstil und Denkkollektiv (1935), in which he identifies the social nature of scientific knowledge production. We all are members of various thought communities (not just scientific ones) and cannot abandon all of them, since each of these has its own way of making sense of and describing the world. Scientists, for example, might in their work life be part of a scientific thought community that shares a particular, rationalistic concept of life or biology, but in their private life they might identify with another thought community that emphasizes subjective experiences over rigid dogmatism. On a side note: These "thought communities" could also be a valuable short-hand to understand how mistrust in science and the 'establishment' interact with 'filter bubbles' in social media and conspiracy thinking. 
another" (Erickson $33 ; 70) .{ }^{7}$ Changes in the "language-game" bring with them new linguistic concepts, different connotations, abstractions and simplifications. Knowledge gained in one community will have to be adapted to the experiences and insights of the wider community, using language that is familiar, relatable, trusted. ${ }^{8}$ These processes of translation affect the interactions with knowledge and its production in both directions: On the one hand, in the scientific community simplified, adapted concepts might shape how (further) knowledge is produced. Ludwig Wittgenstein's ideas about language - more specifically his investigation into how the use of the term "natural law" shapes the research that is being conducted - come in handy here: They tell us that how we talk about science changes the way we perceive it (Wittgenstein and Klagge 430). The language we use is not a "perfect tool for describing and explaining the world", but "something that actually hides things from us, confuses us, leads us astray" (Erickson 17-18). The simple act of using specific words, concepts and frameworks shapes how scientists carry out their investigations. It makes them look for certain things, shapes their thinking in certain ways and thus determines the results they receive.

On the other hand, the translation of scientific knowledge often has the goal of increasing the scientific literacy of the wider community. Most commonly, being 'literate' is used for people who are able to read and write, and thus denotes some form of competence in a specific area, some form of proficiency or expertise. But with the increasing dominance of science and biology in social and political contexts, the concept has since the 1980 s and 1990s also been used in the context of high school and college science or biology education: Educators had realized the importance of students being "scientifically literate" in order to fully grasp scientific developments and take part in social discussions. Gordon E. Uno and Rodger W. Bybee, for example, in 1994, developed a concept of biological literacy that rather than seeing it as an end point - to either be achieved or not - defines biological literacy as a continuum on which individuals are positioned (553). This continuum for Uno and Bybee ranges from nominal, to functional, to structural to multidimensional - from a naïve understanding in words only to a complex understanding of biology as a discipline and its interactions with society (554-56). ${ }^{9}$ For them it is essential that students learn to think creatively about biological concepts,

The form that this translation takes depends on the medium: specialized newspaper articles will use a different language - and require a different translation - than audio-visual use in popular culture, films and TV. The form that scientific knowledge takes in the public sphere can thus differ widely, depending on the intended audience.

More specifically, such processes of translation are often also needed in the scientific community itself. With various fields of specialization, findings from one field are not necessarily understandable for another, even in the same general discipline.

9 In more detail, Uno and Bybee write: "A biologically literate individual should understand biological principles and major concepts in biology, the impact of humans on the biosphere, the processes of scientific inquiry, and the historical development of biological concepts. He or she should develop personal values regarding scientific investigations, biodiversity and cultural diversity, the impact of biology and biotechnology on society, and the importance of biology to the individual. And he or she should be able to think creatively, formulate questions about nature, reason logically and critically, evaluate information, use biological technologies appropriately, make personal and ethical decisions related to biological issues and apply biological knowledge to solve problems." (Uno and Bybee 553). What is interesting is their focus also on the interaction between biology 
critically examine knowledge claims and gain a good understanding of basic scientific principles (553). Uno and Bybee argue that true biological literacy requires more than school or college learning and extents over the whole life and lifespan of students; it is a skill that should be honed and fostered in schools and colleges in order to instill in students a keen interest in biology that they can pursue also after leaving the education system (557). This approach, I think, is particularly relevant as it allows people to gain literacy in a much more sustained way needed for a field that changes as fast as biology. At the same time, it means that in order to be biologically literate, in this definition, a certain consistency in engagement with biological concepts is needed. The 'education' or rather critical examination cannot end after formal education has ended. To be scientifically literate, thus, is not so much concerned with content as with methods, processes and approaches and thus encompasses knowledge, understanding as well as evaluation. A scientifically literate individual should understand how scientists 'do' science and what scientific concepts and basic scientific principles mean, but they should also be able to put results into perspective and make informed evaluations, personal and ethical, about applications of biotechnology. ${ }^{10}$ It is here that discussions of scientific literacy have often drifted towards questions of citizenship and social participation. A society so dominated by science needs more "bodies who know" (Steinberg 29), it has been argued, especially because the stakes are rising with more and more sophisticated and invasive technologies. For further references, I use biological and scientific literacy almost interchangeably, regarding biological literacy, following Uno and Bybee, as a subset of scientific literacy. Both have as their goal a more differentiated competence to investigate and evaluate scientific knowledge claims more generally.

From a Cultural Studies perspective, the media of a culture can certainly be used to facilitate some of this scientific literacy in the broader population, thus reducing the imbalance of power and creating a more egalitarian approach to knowledge production. Popular culture might be especially impactful here due to its ubiquity in everyday life. In his examination of the public perception of science and scientists in popular culture, A. Bowdoin van Riper argues that because popular culture is "more pervasive, more eye-catching, and (with rare exceptions) more memorable," it is in all likelihood more successful in shaping people's understanding of science than formal science education (1104). But scientists often dismiss popular culture representations as unrealistic, excessive, not truthful, unimportant for their own work. Van Riper argues that if popular culture is to serve as a potent "shared frame of reference" for the communication of research results, scientists need to take it more seriously, both as a form of entertainment - or infotainment - and as "a springboard for dialogue with the public" (1107). Formulated in 2003, this argument still rings true today. Where I disagree with van Riper, however, is in his assertion that popular culture can provide a "teachable moment" in which scientists can reinforce their concepts (1107): This assertion positions lay public

and society and the personal consequences of (bio)technologies as well as ethical decisions about their use.

10 This is all the more important because scientific findings and data always need to be understood in the specific framework of how they were generated, with all the limitations and contingencies that entails. 
and expert scientists in imbalanced power relations and thus reflects an outdated division into spheres of competence. Instead of seeing this relationship as a two-way street, it enforces a one-way, paternalist relation that undermines the very idea of a sustained dialogue about the fears, anxieties, concerns and hopes expressed in relation to science and biotechnology. These can, however, find their expression in a popular culture in a different guise: more personal narratives and stories. Listening to medical stories, as the Medical Humanities have shown, can make individual experiences more relatable, create social impact and empathy. Narratives and language, those tools traditionally used to make complex processes more graspable, can provide exactly the platform needed to make sense not just of scientific knowledge but also of its repercussions for one's own life.

\section{Role of Language and Narrative}

Studying such narratives can reveal the structures through which the public makes sense of new interventions, technologies and innovations. Language and narrative, it becomes clear, play a crucial role in the production and perpetuation of biological knowledge through media and the channels of culture. As Lupton writes, we can use the study of discursive tropes - patterns of words, figures of speech, concepts, symbols, ways of describing, categorizing, making sense, implicit values and judgements to show the intertwined, mutually reinforcing relationship between discourse (the "way we speak or visually represent phenomena") and practices (actions, activities around the phenomena) (Medicine 18-19). For example, how we represent diseases can impact how we react to and interact with those affected: Multiple Sclerosis, as one example, often is represented through visual aids such as crutches or wheelchairs. This type of representation, however, risks negating more "invisible" forms of the disease, those that do not explicitly leave marks on the body while at the same time marking people with MS as differently abled, potentially influencing how others react to the diagnosis and in interactions with those affected. ${ }^{11}$

As we can see, discursive approaches are important because people construct their understanding and beliefs about medicine from personal experience, interaction with others as well as with cultural products: Mass media and narratives become influential ways of shaping perceptions, contributing to people's understanding of medical phenomena. They provide a medium through which we can access socio-political changes and challenges, structures of feeling, prevalent beliefs and values. Stories register and transmit cultural assumptions, concerns and anxieties as well as norms and values across cultures and time periods (Wald and Clayton viii); they can be seen as textual

11 My master's thesis dealt with exactly this ambiguity in the representation of invisible disabilities, more specifically Multiple Sclerosis (MS), in American popular culture. MS, as a largely invisible illness, becomes visible, is made visible through conscious acts of translation and representation. These representations can have an impact on how individuals experience disease and how society reacts to them. I argued that the most productive representations of MS make use of in/visibility, being both visible and invisible at the same time, to portray MS as the highly fluctuating disease it is (Grewe-Salfeld, A Dialectic of (In)Visibility: The Cultural Imaginary of Multiple Sclerosis, 2016, unpublished). 
representations of current concerns. We can therefore use narrative to understand the present, to show how the contemporary world was and is made into what it is, in order to shape the future: Narrative makes visible some of the broader concerns in contemporary culture, not just but also related to biomedical technologies. Analyzing the stories of illness and health, as it is done in the Medical Humanities, can elucidate how people make sense of their experiences in the medical encounter, how they construct their identity and frame their confrontation with their own biology. The in-depth study of texts that is crucial to discourse analysis in narrative medicine can therefore be seen as a means to analyze changes and social processes because texts - broadly understood in Cultural Studies - can gives us access to the lived experience of everyday life. A contemporary example is the movie Still Alice (2015) that shows a woman in her early 50 s tackling a diagnosis of early-onset Alzheimer's, providing insights into the firsthand experience of the disease and its effects (Glatzer and Westmoreland). ${ }^{12}$ Many more of such illness narratives can be found in culture, many of them following similar structures, as Arthur Frank pointed out in his seminal text on the Wounded Storyteller (1995/2013). ${ }^{13}$ Renditions of such narratives can be found in contemporary social media such as Facebook and Instagram as well. These platforms make the sharing of such personal narratives of disease much easier for individuals, allow their voices to be heard in a wider audience and shared in 'their' (biosocial) community. Such accounts can give patients agency and authority over their own experience (cf. Hinson and Sword).

Scientific and biological information circulates through language, images, stories and thereby is part of the circuit of meaning production in popular culture: the vocabulary used, the norms espoused, the judgments made, in this context shape the public understanding of science. Nancy Tomes, for example, traced how fears and desires were used by journalists, advertisers and in the products of popular culture to turn epidemics into entertainments ever since the beginning of the $20^{\text {th }}$ century: Her examples include more common diseases such as Tuberculosis - or more recently AIDS - as well as unusual diseases like Parrot Fever, that contributed also to the dispersal of scientific innovations and information into the public sphere. ${ }^{14}$ Disease, and with the

12 Still Alice, the movie, is based on an eponymous book written by neuroscientist Lisa Genova inspired by the Alzheimer's diagnosis of her own grandmother. After the success of Still Alice (2007, on the New York Times bestseller list for 40 weeks) Cenova has continued to combine her neuroscientific education with her literary talent to write a series of books about people with neurological diseases or problems. The movie received an Academy Award for Best Actress in 2015.

13 In The Wounded Story Teller (1995/2013), A. W. Frank distinguishes three different types of illness narratives: The Restitution Narrative, in which the patient is restored to health through science and medicine; the Chaos Narrative, in which, often retrospectively, illness is narrated as an interruption that lives from the silences and disruptions that illness (or the impossibility of putting its experience in words) entails; and the Quest Narrative, that describes the experience of illness as a journey, also of personal transformation.

14 Tomes claims that disease and epidemics have gained a new cultural visibility due to modern communication media and the new globalized threat they pose. The templates of disease used in contemporary accounts of germs, viruses and epidemics, however, are traced to developments in the early $20^{\text {th }}$ century and the parallel rise of the germ theory of disease and new mass media. In news media accounts and journalism, in particular, the increasing use of stories of disease also served to perpetuate scientific facts out of the laboratory or clinic into the households of the masses. More- 
increasing sway of science scientific explanations and technologies as well, have always been part of the cultural imaginary, the narratives and representations circulating in a culture. This is not just true for technologies that already exists today but also for those of tomorrow. Narratives in novels, films, or visual media readily dramatize possible implications of discoveries and interventions, and thus provide a good medium to think through technologies before they are developed or widely in use. Concerns about science often arise out of "popular science predictions and speculations" about advances that are "just around the corner" and are thus not based on real experiences and evaluations (Rose, Politics of Life 78). Rather, we find a recourse to speculative narratives and science fiction when the future of biotechnology and biomedicine is discussed. Much of this is left to our imagination. Technological imaginaries as they are developed in science fiction do not just speculate about the future on the basis of current technologies, but also tell us a great deal about the fears and concerns connected to technological advancements. Technological imaginaries influence the public understanding and response to biotechnology and create fears and expectations about the future (Stevens 269). In fact, the struggle over the popular imagination - genetic imaginaries, biological imaginaries, cultural imaginaries - can be understood as a larger struggle about the future: What is imaginable, in the most literal sense, becomes desirable and doable for some or objectionable and deterrent for others. In either case, it can shape popular attitudes and judgements about applications of biotechnology and thereby change their prevalence and use.

When what is imagined transcends what is currently feasible, such imaginaries can also serve as imaginative prompts for further research and development. The imaginaries about science created in popular culture shape not just the public understanding, reception or response to biotechnology: Films and biotechnological imaginaries play a role in shaping technologies and research as well as their use and acceptance. Such linguistic and visual representations of science in culture are always political and reveal the affective structures behind the acceptance or disapproval of biotechnologies in society: They serve to reinforce dominant bio- and body politics, ideologies and norms at the same time as they reveal deeper cultural anxieties about the (future) health and state of the corporeal body, the expanding medical control of bodies, the specter of categorization, deviance, and "newgenics" (cf. Lupton, Medicine 78). Imaginaries, moreover, create and perpetuate promises about the (future) state of the body, its potential for modification and optimization. Through this type of imaginative engagement through language, we can see how culture and technology are intimately related. In "complex feedback loops" culture and technology constantly remake one another (Stevens 5).

\section{Science and Culture}

What is needed, then, is to culturalize science and biotechnology, to subject them to careful cultural analysis of their epistemic practices, to make these practices visible as

over, the portrayals of disease, she writes, also were closely aligned with rising consumer culture: Narratives of disease were not just a source of cultural anxieties but also highly profitable, selling cultural productions, stories and products - selling disease (cf. Tomes, Epidemic Entertainments). 
cultural, to show that their claims are premised on categories that are cultural, historically and locationally diverse and contested (cf. Steinberg 155, Kunow). Science is generated in and from culture - "from language and discourse, actions and practices, representations and material cultures" - and "spread through our society and culture, unfolding in multiple domains and in multiple forms" (Erickson 1). (Popular) culture here opens up as a particularly influential and contested domain. It is influential because it has a high impact on individual and collective judgements, contested because its position is not universally acknowledged, especially by scientists, because much of what it produces seems to violate the norms and standards of the scientific method. Still, according to Stevens, popular culture, more than anything, feeds the hype around biotech by fueling expectations and foreshadowing the future. The scientific and biotechnical imaginaries created in popular culture influence the direction of research and therefore how biotech develops. But most importantly they influence how people understand and use science (8). As affective structures, "hype, promise and fear," Stevens claims, need to be part of the cultural analysis of biotechnology (8-9). Here, we begin to leave the realm of constructivism and move into considerations of the material nature and multiple assemblages present in and shaping our engagement with biology.

\subsection{Promissory Bodies and Plastic Life}

Attention to the material conditions of life and knowledge production has in the past years been more and more acknowledged as the basis for an extensive critical analysis of culture. As a result, the body has increasingly moved to the forefront of academic analysis: Its lived experience, embodied reality, use and abuse in culture have come to be favored topics of research. In a first 'turn' towards the body and its experience, researchers argued that the social body would not exist without the natural, biological one and that they both influence each other. The body became political and symbolic, a contested terrain but also a fundamental aspect of cultural-critical inquiry. Many of these cultural approaches to the body looked at how bodies gain symbolic significance through cultural construction. The concept of the socially and culturally constructed body, perpetuated in such theoretical engagements, variously positioned the body as determined by language, shaped by discourse and representation which are historically and culturally contingent (following Foucault) or produced at the intersections of power relations. Traditionally, social constructionism with its high emphasis on language, meaning, and representation tended to subsume nature into culture, to give culture power over nature. The body was inscribed by culture, a surface to be read and coded, a place where social relations are manifested (Pitts-Taylor, Cultural Encyclopedia xxii-xxiv). The body became a screen, a blank piece of paper onto which cultural discourse writes its story - what is materially there, and how that shapes the story to be told was less important. The materiality of the body, its biology, had no agency in this process, it is a passive recipient instead of dynamic. Such a constructivist critique is useful, even necessary, to counter determinist and reductionist claims, to highlight cultural and historical diversity of experiences as well as the social organization and basis of inequality (Pitts-Taylor, Brain's 7). While it was certainly also a big influence on my 
considerations in this book, it is, nonetheless, no longer enough to adequately capture the lived reality of embodiment today. What is needed (in addition) is a new appraisal for the material basis of life, of bodies, and how it interacts with social constructivism, with culture and meaning ascription.

In the last decades of the $20^{\text {th }}$ century, and the first decades of the $21^{\text {st }}$, academic considerations of human life have begun to enrich concepts of the body as socially constructed and culturally malleable with more and more in-depth considerations of the body in its materiality. Scholars increasingly turned their interest towards how people experience their own bodies, on the phenomenology of individual embodiment (PittsTaylor, Cultural Encyclopedia xviii -xix). ${ }^{15}$ What is often termed "new materialism" - in Pitts-Taylor's words, a "reaction" to the focus on linguistics, discourse and representation in the $20^{\text {th }}$ century ("Mattering" 2 ) - has its theoretical roots most prominently in feminist and queer studies. ${ }^{16}$ Feminist scholars have long addressed the complex relationship between the material body and its social enactment or performance, especially in the context of sex and gender. ${ }^{17}$ Younger (feminist) theories show the body as defined by multiplicity and potentiality, such as two scholars who have been influential for my considerations, Bernadette Wegenstein and her discussion of body modifications and the cosmetic gaze, most prominently in Getting Under the Skin, and Victoria Pitts-Taylor's theorization of brain plasticity. ${ }^{18}$ According to Marc Chrysanthou, this "bodily turn" in contemporary theory is symptom of the wider cultural fragmentation and disorientation in postmodernity - creating a "disorientating ontological insecurity that predisposes the postmodern self to take uneasy refuge in the most basic shelter of all: his or her own body" (470). The body, in lieu of other forms of safety, security, or anchoring, becomes the primary target of the self, a safe harbor but also the last frontier that can be controlled or shaped by the self (even though it does not fall outside the realm of social control). According to influential theorists like Anthony Giddens or

15 Often the basis for such thinking is a renewed interest in phenomenology, along the lines of Merleau-Ponty, as Wegenstein argues. Merleau-Ponty stresses the "interdependence of body and world," where the world is experienced through the lived body. Wegenstein writes: "Whether maintaining a constructivist, a performative, a volatile, or even an essentialist account of the body, all of these ways of thinking of the body presume that the body is access to the world (given, construed, performed, or even all at once)" (Cetting 30-32).

16 A key text on new materialism is Coole and Frost's New Materialims. Ontology, Agency and Politics (2010), in which they collected a range of critical works that exemplify the new engagement with matter, materialization, human embodiment, agency and our material surroundings.

17 Most prominently ]. Butler in Gender Trouble argues that the biological, material body is shaped through the performance of gender, that the body becomes a process of enactment.

18 In their accounts matter is not passive (recipient of social meaning and social constructivism) but dynamic - having agency of its own, always interacting with the social and cultural. This shift leads to a renewed understanding of the relationship between nature and culture: It shows that matter and culture determine each other, are not separate or even separable, but in constant interaction, imbricated in a network of different actors, forces, and affects. According to Nick ]. Fox, the ontological focus changes from single "entities to relationality." Rather than focusing on what human bodies and identities are, consideration is given to the networks and assemblages they are part of, to flows of affect in social production rather than solely human agency (N. J. Fox 138-39). 
Chris Shilling, the body has become a space of self-expression, a project to be worked on as part of one's identity formation.

This is a very much future-oriented and -directed view of the body, concerned with the potentiality - or if we wish, the promises - embedded in its materiality. In postmodern culture, Victoria Pitts argues, the body is no longer seen as "fixed and pristine or as subordinate to the self;" instead it is seen as open to manipulation, in its aesthetics, norms and possibilities. The body becomes open for new symbols, meanings, options, she claims, often involving temporary or permanent modifications of the body through hard work, cosmetics, chemicals, or technologies, all of which somehow sculpt, transform, reshape it in some way or other (through painting, cutting, implanting, transplanting, extending, lasering, suturing) (29-30). This openness to modification - in the present, for the future - through technology has changed how we think about what it means to be human, what it means to have a body. The bodily turn in theory has disseminated new means of theorizing human embodiment, identity construction and the lived experience of our materiality. In this subchapter, I want to delineate and focus on four of them: the somatic self, the concept of life and the body prevalent in contemporary discourse, the idea of plasticity, and theorizations of affect.

\section{The Somatic Self}

Interest in the body is also very much present in culture and society at large. The body has become a focal point in questions of identity, normalization, control, self-expression, and optimization in the name of the (global) market. According to Brian Turner since the second half of the $20^{\text {th }}$ century we have lived in a "somatic society," a society in which the majority of problems - moral, political, social - are framed in and through the body. A major driving factor for Turner are the socioeconomic changes connected to the expansion of medical technology and discourse to more and more areas of life: In this way the body was again and again framed as biological, in need of medical intervention, but at the same time subject to economic forces. According to Rose, we sense ourselves as "biological creatures, biological selves" whose vital existence is increasingly subject to (self) control and exploitation in the contemporary molecular politics of life itself (Politics of Life 4). This view of the body and self as inherently biological leads to an intrinsic connection between bodies and selves: the self becomes the body, meaning that work on the body equals work on the self, that separations between inner and outer, mind and body dissolve.

Selves today are embodied and biologized (Davis and Morris 418) and in consequence questions of identity are no longer seen as separate from the body. The individualization of bodies, historian Norbert Elias argues, had begun in early modernity, when with the separation of individuals from a broader social body a concomitant process of differentiation took place. Instead of an undistinguishable mass of people, individuals, their bodies and problems slowly began to take center stage: medicalization, especially the medical gaze developed throughout that time, focused on the body at hand - the body on the table - and "dissected" it with a view to individual causes as well as socioenvironmental factors. The individualization of life (choices) then cannot be seen outside of the individualization of material bodies. In this way, individual bodies were un- 
derstood in biological terms and any form of intervention acted on the biological body. Similarly, according to Anthony Giddens, modernity led to a pervasive sense of ontological insecurities by cutting self-identities loose from traditional (community-based) forms of identity (Modernity and Self-Identity). Identities became destabilized. Shilling, taking up Gidden's theme, argues that the response of the self to this uncertainty is to "refocus the source of identity and meaning on the body" (Chrysanthou 470-71). The body, thus, in postmodernity has become a primary source and expression of individual identity. ${ }^{19}$

As Rose writes, we increasingly relate to ourselves as "somatic individuals:" "as beings whose individuality is, in part at least, grounded within our fleshly, corporeal existence," whose body is a "key site for work on the self" (Politics of Life 25-26, 18). The body has been framed as a "project" to be worked on as part of the construction of individual self-identity (cf. Shilling 5). All around we are confronted with perpetuations of this demand for self-realization through the body (Wegenstein, Getting 4-5). From official discourses of health promotion to mass media or popular accounts of disease and health, dieting and fitness regimes, we are urged to act on the body, to construct and reconstruct it (Rose, "Politics" 18). "Our bodies have become ourselves," writes Rose, they are "central to our expectations, hopes, our individual and collective identities, and our biological responsibilities" (Politics of Life 105). This "somatic self" goes hand in hand with the (market) ideal of the autonomous individual of Western liberal democracies: this self is "free yet responsible, enterprising yet prudent, conducting life in a calculative manner by making choices with an eye to the future" (Rose, Politics of Life 111). The consequence of this somatic individuality is also an increasing responsibilization of this individual for their health and well-being. The somatic self, in the end, is the one that makes decisions about their material body and vital existence, a body that is increasingly seen as in itself malleable and fluid. This somatic selfhood, thus is both liberating and highly restrictive, luring with its emphasis on choice and autonomy but also oppressive through new webs of control, responsibility and domination - question that will come up in the subsequent subchapter once more.

\section{A New Body Concept}

How then do we conceptualize a body, how do we approach bodies, in a world that puts as much emphasis on them? Both in the social sciences and cultural theory and in the biological sciences organisms today are seen not as singular and universal, but as varied, plastic, part of dynamic systems and environments (Pitts-Taylor, Brain's 7). Our understanding of the body through its biological materiality, thus, is characterized by a new focus on interactions and situatedness. The concept of the body, used also in discourses of DIY biology and medicine, however, is not only a reflection of this new dynamic view but also incorporates older, deeply engrained views of the body. In the following, I will briefly introduce different conceptualizations of the body - from the mechanistic view and the "body-in-pieces" (Wegenstein), towards the "biomediated" 
(Thacker), commodified and malleable, posthuman body - and then delineate how the techniques and technologies of DIY biology and medicine make use of them.

Bodies historically underwent a process of fragmentation: They were divided into individual "body parts," individual processes and structures to be studied and described, delineating the body into ever smaller chains of cause and effect. Much of this process was dominated by a mechanistic language. Following Lupton's narrative in Medicine as Culture, the conception of the body as a form of "machinery" is one of the longest-lasting approaches to the body, that is very much reflected in discourses around the body until today: Mechanical metaphors, such as clockworks or hydraulic machines, are often used to convey the inner workings of the body. The heart as a pump is probably one of the most salient one of these. Mechanistic views restore a sense of order, rationality and predictability in a highly complex and hardly understood organism. By implying that body parts can be as easily replaced as machine parts, however, this view of the body places more emphasis on treating the problematic part rather than looking at the system as a whole. During the past decades, machine metaphors have largely been replaced - to the same effect - with metaphors of the body as a computerized system: examples here include the view of the brain as a form of information storage or memory hardware and the bio-informatic metaphor of DNA as a genetic "code:" "They are reworkings of the mechanical metaphor in representing the body as being comprised of a multitude of tiny interchangeable parts, rendering the body amenable to objectification and technological tinkering in the interests of developing the 'perfect' human." (Lupton, Medicine 59-61) According to Stevens, biotechnology is premised on this idea that "understanding life requires understanding it on a submicroscopic level," that it "works something like a machine: it has moving parts that can be taken apart, modified, and put back together again" (345). Stevens stresses that such a view of life "suggests particular ways of tinkering with and thinking about organisms," of engineering life itself (345). ${ }^{20}$ The body, in this mechanistic view, is reduced to individual parts: from limbs, to organs, to molecular structures - it becomes a 'molecular body.' This perception of the body, however, "as mechanical device or a 'human motor' which can be controlled and regulated by biomedical interventions" has been criticized by social historians of medicine already since the 1970s (J. Tanner 38). It is regarded as simplified and indeed potentially limiting through its reductionism (Nesse). The ongoing use of mechanistic metaphors of the body, however, have engrained such a view and given it currency. ${ }^{21}$

In Bernadette Wegenstein's concept of the "body-in-pieces" the body is split into parts as well, but she complements this reductionism with a wider view. Wegenstein argues that since the anatomical fragmentation in the $15^{\text {th }}$ and $16^{\text {th }}$ century, but especially

20 The question then, is what does the view of the body as mechanistic, as a collection of parts - or in the more reductionist language of biotechnology, a collection of cells and molecules - to be changed, enhanced, modified, mean for subjectivities of people? The biotechnological premise of the mechanistic-computerized body requires the subject to change their conception of their own body: In order to use and accept such techniques and technologies, I need to adopt this view and think differently about my own body.

21 Also in seemingly 'progressive' ideologies such as transhumanistic ideas, humans are often seen as "no more than a sum of their physiological processes, which are entirely mechanistic, knowable, and controllable" (Tirosh-Samuelson n.p.). 
with the constitution of the gaze of medicine since high modernity, the body concept has changed from a unified perception to the "body-in-pieces," fragmenting and decomposing the body into ever smaller and more controllable units that are perceived as working independently from each other (Getting 3-8). Towards the end of the $19^{\text {th }}$ century, she claims, a more holistic view of the body emerged - "defined as the interrelation of all (body) parts-in-pieces" - in the end leading to the incorporation of early modernity's fragmentation into a holistic view of the body (Getting 24). The bodyin-pieces is thus characterized by a fluctuation between fragmented and holistic body concept, "... a dialogue between the body as a whole and as a multiplicity of fragments" (Getting 35). In the practices under examination in this book, especially those targeting the human body, we can see a similar fluctuation between and integration into each other: While the fragmented body-in-pieces - or the 'molecular body' - is the basis for much intervention (into single, molecular units), these interventions target the body as a whole - a holistic view of the body that also incorporates notions of wellness and psychological health. Through its fragmentation, it is believed, the body can be made 'whole' again.

Contemporary technologies, however, pull our body concept into even more complex directions. Eugene Thacker develops the concept of the "biomediated body" or informational body to address the increasing mediatization of the body. For Thacker, the body is both a medium ("a means of communication") and mediated ("the object of communication") (Thacker 55-56). ${ }^{22}$ The mediated body seems to be a bit more approachable than the idea of the body as a medium. For Thacker, the body as a medium means that the "techniques and technologies are not external to or qualitatively different from the body," not developed to operate on or control the "natural/biological" world but generated from within: In biotechnology, the body becomes the technology or instrument, the medium, when "the material components and biological organization of the body" are "rematerialized, renormalized, even redesigned" (Thacker 57-58). Tacking up Thacker's concept, Patricia Clough argues that the biomediated body is informational: it "exposes how digital technologies, such as bimedia and new media, attach to and expand the informational substrate of bodily matter and matter generally" (Clough 207-08). The body here is seen as information and data, a view that also rests on the idea of DNA as code. Such a biomediated, informational body raises another specter: What happens when the body becomes not just an assemblage of disconnected, repairable and replaceable - but still material - parts but an (impersonal and immaterial) set of data and information? Also in DIY biology, especially when it comes to tests for bodily conditions, the simple availability of information can already have an immense impact on the body.

In all this, it should not be forgotten that due to its embeddedness in global capitalism, the body has also become commodified. Already in 1994, Deborah Lupton notes that the body has become a "consumer commodity which must be groomed to achieve maximum market value" (36). ${ }^{23}$ The narcissism in Western societies with its "obsession with

22 Apart from biotechnologies, Thacker's examples include older techniques of the body as well, such as fashion, tattoos, cosmetic surgery, body building, or cyborg performances ("What is Biomedia" 55-56). 
physical appearance, the purchase and consumption of commodities and the construction of self-identity" means that how people define themselves is closely linked to how and what they consume (36). One of these new commodities is the body. Bodily maintenance has become an area of discipline and control: In order to market it well and maximize its efficiency, servicing and care for the body, often through lifestyle adaptions, is needed. The obsession with youth and beauty as features of desirable bodies have generated a whole industry devoted to bodily maintenance. This notion of the body reinforces the idea that the body can be reshaped to fit accepted notions of appearance, productivity and efficiency (Medicine 36-38). Stefan Selke likens this view of the body to Bourdieu's notion of capital. According to Selke, as a project the body is endowed with a new status: health is turned into an alternative religion, the body into a lifestyle product and "temple." As such, the body becomes a new form of capital: to others and one self, health and fitness signal discipline and attractiveness, which equal status and power in society. As Selke argues, one has to "invest" in this capital both physically, psychologically and materially because health is needed to make full use of it. The individual is turned into a "manager" of her bodily capital. ${ }^{24}$ Correspondingly, the body is seen as a resource that can be worked on and with - in Selke's words "bearbeitbares Material." This implies a mechanistic and functionalist view of the body, one in which the body can be taken apart and repaired, either self-directed or in what Selke loosely terms "body workshops" (my translation). In this view, the human is no longer a subject but an object, life a project in which the investment in body capital becomes a daily, individual task ("eigenverantwortlich"), complete with sanctions for non-compliance (Selke 5960). Today we "replace our identities [and bodies] with market-driven, slightly altered, and improved ideals of ourselves" (Wegenstein, Cosmetic Gaze 125). Biotechnologies have turned life itself into "a productive force to be harnessed, technologized, and capitalized," and thus become part of the circuit of capital and its regimes of speculation and risk (Stevens 77). This commodification becomes explicitly present when talking about body modifications and enhancements and should thus be mentioned here. As Debra Shaw claims, "[a]s life itself, understood as bio-genetic information, becomes commodified, it equally becomes manipulable and hackable" (289). This view, of course, is also foundational for the practice of 'body-hacking' under consideration in this book.

Posthumanist thinking takes up this notion of malleability and potentiality of the body. In her treatise on How We Became Posthuman, N. Katherine Hayles argues that it is exactly the acceptance of a malleable, manipulable body that heralded the era and rise of the "posthuman." She argues that we have become posthuman in the moment the results of the Turing test were accepted: This test set out to discern whether or not people could differentiate between artificial intelligence and human intelligence by symbolic (59-60). Body positivity can be regarded as a 'bottom-up' counter movement against such beauty and performance standards. Body positivity promotes the acceptance of all bodies, regardless of physical ability, size, gender, race, appearance. Currently, a new wave of body positivity is promoted and critically discussed on social media - in response also to discriminating beauty standards on social media - and used in marketing campaigns. 
"talking" with the other (computer or human) through questions and answers. Recounting Hayles' argument, Christina Lake summarizes that not the test itself or its results were important, but how it was framed: Following a Cartesian dualist thinking of mind and body, it favored information/mind over embodiment. The body here becomes "the original prosthesis we all learn to manipulate, so that extending or replacing the body with other prostheses becomes a continuation of a process that began before we were born" (Hayles 3). The body is simply a resource to be manipulated, it is plastic, can be reshaped with whatever enhancement technologies are available (Lake 14).

All the ideas mentioned above, I argue, come together in how we frame the body in DIY biology and medicine, but also culture and society at large. The contemporary concept of life and of the body - as it is embodied in the cultural representations and material practices under examination here - is in its basic convictions still reductionist, meaning looking for the smallest common denominator, but also mechanistic-systematic. What do I mean by that? Contemporary discourses of the body see parts of life and parts of the body as both exchangeable and repairable and as embedded in larger networks or systems. They highlight their complexity and malleability at the same time. This view is, taking inspiration from Wegenstein's ideas on fluctuation, produced by the incorporation of mechanistic thinking into systems theory and network thinking. DIY biology relies on a view of life as rearrangeable (mechanistic) but has also incorporated more complex, even holistic ideas of the body (systematic). The result is a mechanisticsystematic, highly malleable concept of the body.

\section{From Brain Plasticity to the Plastic Body}

The notion of plasticity summarizes much of what has been said before in a neat new concept. With the reconceptualization of the brain as constantly adapting and the rise of epigenetics, plasticity has emerged as somewhat of a new master narrative in the natural sciences. More and more bodily processes are no longer seen as fixed but as everchanging and adapting through outside influences. ${ }^{25}$ But the triumph of plasticity has not stopped there: befitting of our current demands of constant mobility and change, it has also been adopted from the realm of biology and applied to social and cultural questions - again situating biological processes in their time and place, linking nature and culture.

Originating in $18^{\text {th }}$ century materials science, the term "plasticity" was originally used to describe the malleability of matter (in the broadest sense). During the $19^{\text {th }}$ century, then, it shifted towards a biological meaning and was used to describe the ability of organisms to adapt when faced with environmental changes (Berlucchi and Buchtel). Following this line of thought, the idea of plasticity was often applied in the context of cell culture and later brain science. As Hannah Landecker delineates, after the rise of cell culture - the ability to grow, divide, multiply cells in vitro, to create cell lines and

cf. for example Victoria Pitts-Taylor on the plastic brain in The Brain's Body (2016), Pitts-Taylor on our contemporary engagement with matter in Mattering: Feminism, Science and Materialism (2016), Hannah Landecker on cell culture (2005), Berlucchi and Buchtel (2009) on Neuronal Plasticity or Andy Clark on plasticity and embodiment (2007). 
work with them - "being a cellular entity ... means being freezable and open to artificial synchronization; any live thing made of cells, after these interventions, becomes an object that can be stopped and started, suspended and accelerated" (n.p.). The plastic matter of the organism, cells and with them their accumulation in the form of bodies, has come to be seen as manipulable through (human) intervention. For her, then, the history of biotechnology in the $20^{\text {th }}$ century is mainly characterized by the "increasing realization and exploration of the plasticity of living matter" (Landecker).

This exploration of plasticity has in the past decades reached the brain: In contrast to older views which saw brain development as stopping during the first decades of human development, contemporary narratives frame it as constantly creating new synaptic connections, modifying itself in response to outside and inside influences. In her recent (2016) examination of the corporeal politics of brain science, Victoria Pitts-Taylor closely examines this notion of neuronal or brain plasticity. In Deleuzian language, she claims, brains are "biological becomings, always in process, always open to transforming themselves and being transformed" (Brain's 17). This openness, as a next step, allows us to transform who we are, gives agency both to people and matter (Brain's 17-18). Plasticity thereby denotes both the brain's material potential and the possibility for modification and enhancement (Brain's 24). Moreover, with the advances in genomic knowledge since the publication of the Human Genome Project at the beginning of the $21^{\text {st }}$ century, genetics came into the purview of plasticity as well. Epigenetics describes the process by which genes are expressed - 'switched on' or 'turned off' - through molecular processes as a reaction to the intracellular environment and external environmental factors. Epigenetics stresses the plasticity of genetic matter and is, like brain plasticity, a "medium through which social and environmental experiences materialize at the molecular level" (Pitts-Taylor, "Mattering" 5-6).

Plasticity, thus, can be seen as an umbrella term for all sorts of biological processes, one that is procedural in that it connotes a basic principle or process rather than the thing itself. Correspondingly, the notion of plasticity lacks a clear-cut definition; on a basic level, however, plasticity is used to describe the processual nature of events, entities and bodies, their state of perpetual becoming, their ability to change and be changed. ${ }^{26}$ Plasticity can be seen as the material potential of living organisms in general. ${ }^{27}$ When defined as plastic, living bodies (human or animal) are thought to possess an ongoing capacity for renegotiation (Clark 268). Plasticity highlights the contingency of biology and embodiment, and thereby also obliterates the distinction between nature and culture. According to Pitts-Taylor, the plastic brain challenges the old separation of mind and body, culture and nature, by collapsing the distinction between brain (as biological) and mind (as immaterial, discursive, symbolic) (Brain's 4-5).

26 Other biological examples of plasticity can thus include evolutionary adaptation (though this is a rather slow process of plasticity), or - with a view to genetics - the variability of phenotypes and genotypes in monozygotic twins, how environment, microbiome and epigenetic factors can impact gene expression.

27 As quoted above, Pitts-Taylor, in The Brain's Body, refers to plasticity as the material potential of the brain (24). Given the widespread circulation of the idea of plasticity, I would expand this idea towards (whole) bodies and living organisms more broadly. 
The ideas implied by plasticity - dynamism, interconnection, network thinking to a certain degree seem to be contesting mechanistic ideas of the body. But it should not be forgotten that these ideas were what formed 'plasticity' in the first place. Our contemporary mechanistic-systematic view of life and the body is in its foundation a mechanistic view of life 'upgraded' to fit contemporary network-thinking: it acknowledges both the hardware (the material body) and the software (the network of multiple connections) as being replaceable, repairable, taken apart. Plasticity, then, is a more integrated view of life, one that combines hardware with software and the environment in a fruitful way. Especially for conceptualizations of the body that look favorably at transor posthumanist thinking, plasticity seems to be the way into the future. The view of the body as plastic, for Andy Clark, means that humans are biologically predisposed for recalibration, reconfiguration and extension: "It is our basic, biologically grounded nature...to be open to a wide variety of forms of technologically mediated enhancement, from sensory substitution to bodily extension to mental extension and cognitive reconfiguration." (Clark 278) For him, our basic nature is plastic. Clark here argues for more attention towards the plasticity of embodiment, in the sense that humans are able to incorporate new "equipment" into their "systems," making possible all kinds of enhancements such as human-machine interfaces (Clark 264). ${ }^{28}$ While I would not go as far as blindly espousing transhumanist ideas - they pose their own questions and concerns after all - a view of bodies as plastic shapes our definitions of who and what we are, can or will be in the future. Here, the concept of the plastic body becomes important for DIY biology and self-directed enhancements. According to Nikolas Rose, individuals can today think of their bodies as open to modification: "Our somatic, corporeal, neurochemical individuality now becomes a field of choice, prudence, and responsibility. It is opened up to experimentation and to contestation. Life is not imagined as an unalterable fixed endowment." (Politics of Life 40). In other words, the body as plastic opens many new possibilities of playful and strategic engagements with bodily materiality. But this new form of engagement also creates new forms of responsibility.

This paradigm of plasticity has also found application outside of the natural sciences. For example, theories of neural plasticity underpin the study of affect - the capacity to affect and be affected on levels below consciousness - in Cultural Studies. ${ }^{29}$ The new materialist orientation of social and cultural theory, furthermore, seems to incorporate notions similar to plasticity into its reflections on the relations between humans, non-human animals, things and capital (Pitts-Taylor, "Mattering" 5-6). Plasticity, thus, has also encouraged theories of late capitalism and the global market place. Emily Martin, for example, argues that the global marketplace demands an almost manic form of plasticity, encouraging us to be "always adapting, scanning the environment, continuously changing in creative and innovative ways, flying from one thing to another, pushing the limits of everything, doing it all with an intense level of energy focused totally on the future" (E. Martin, "Mind-Body Problems" 578-79). Likewise, Catherine Malabou describes similarities between the idea of neural plasticity and the flexibility, 
multitasking, and self-modification demanded in late capitalism. Plasticity, she argues, can both mean to "receive form," to be modifiable as clay is, and to "give form," to be formative, as in plastic surgery. The plastic brain can create and receive form but it can also be a site of resistance and destruction: It can take form, give form, and resist form (5-6). ${ }^{30}$ According to Malabou, this idea of plasticity - so pervasive in fact, that we are mostly ignorant of it - has a counterpart, or evil twin, in the economic and political organization of our life: the flexibility demanded in the work place, the ability to modulate oneself, the high importance placed on mobility, adaptability, efficacy, creativity (10). This flexibility, the "ideological avatar of plasticity," she argues, often "superimposes itself" on plasticity, this positive, promissory, liberating idea (12). The problem is that flexibility only captures one side of the meaning of plasticity, "receiving form," not to give or resist form: "flexibility lacks...the resource of giving form, the power to create, to invent or even to erase an impression" (12), rendering people docile and obedient, bowing to the whim of the market and an economy of flexibility without limits. ${ }^{31}$ Malabou here links the brain as a plastic network to the network of current global capitalism, both of which share some of the same core values. Plasticity, in the broadest sense possible, could therefore also be described as a latent cultural condition of our time that extends beyond biology and materiality to society and politics as well.

In the context of biology, plasticity can be problematic as well, visible in what Sigrid Schmitz describes as the paradox of plasticity: It allows us to see the body as biologically malleable and shaped in response to experience (which is positive, also for feminist theorists), but it also describes the body as reduced to its biology, a new form of biological determinism. Here, the question of agency is a decisive factor: Agency in plasticity can either lie with the subject or in the materiality itself. If we see agency as belonging to the subject, this means that for example in brain plasticity the subject can modify her own brain function, for example through technological extension or use of (psycho)pharmaceuticals (Pitts-Taylor, Brain's 21). This reinforces notions of the somatic subject, responsible for the management, optimization and enhancement of her biology in the name of the neoliberal market. ${ }^{32}$ If we see agency as lying in the materiality itself, however, plasticity also carries with it a certain form of defiance: The body being able to change itself without our knowing or 'consent' challenges what we take for granted or wish to have - that we are in control of our bodies, lives, and selves. It brings our plastic embodiment into the realm of contingency and uncertainty so characteristic of contemporary life. This uncertainty, following this train of thought, is also part of the affective structures that underpin (self-directed) interventions into and modifications of the human body.

30 Malabou deduces this destructive aspect from the French plastique or plastiquer - used for blowing up things using plastic explosives (5).

31 To rebel against this culture, to "refuse to be flexible individuals" "who have no greater merit than that of knowing how to bow their heads with a smile," for Malabou answers her opening question: "what should we do with our brain?" (78-79).

32 On the other hand, as Pitts-Taylor stresses, this form of self-directed neuroplasticity presupposes a form of the Cartesian body/mind dualism and sees agency as an exclusively human property (Brain's 21). 


\section{A Short Excursion Into Affect}

Since the publication of Eve Sedgwick and Adam Frank's "Shame in the Cybernetic Fold" and Brian Massumi's "The Autonomy of Affect" in 1995, we find a resurgence of interest in affect in various forms and conceptualizations. Many scholars in Cultural Studies have embraced affect as a new critical trajectory as well: Culture is increasingly analyzed along the affects and intensities within social formations. Particularly in materialist cultural critique of bodies, embodiment and (bio)technologies one cannot really find one's way around theories of affect. Since affective structures of desire, fear and promise are one of the most forceful driving factors behind DIY biology and medicine, it is only fitting that some of the core denominators and relevant aspects of affect theory should be included in the theoretical toolbox developed here.

Since the late $20^{\text {th }}$ century, the social sciences and humanities have experienced what can without doubt be termed an "affective turn," the increasing proliferation of theories of affect in many areas of scholarship. ${ }^{33}$ This proliferation, however, has not led to a single, generalizable theory of affect - the desirability of such a theory remains questionable in itself - but to multiple iterations of the concept in different contexts. What they all share is their historical antecedents and foundation. Theories of affect need to be seen in the context of a wider critique of theory in Cultural Studies: a reaction once more to the (over)emphasis on the representational, symbolic, language, power in poststructuralism, a call for a return to the lived materiality of bodies and individual experience. ${ }^{34}$ It can thus be understood as a critique of and response to poststructuralism, deconstruction and the linguistic turn as well as their perceived limitations and dissatisfying premises. It began to dawn on some scholars that textual approaches cannot fully capture the social world and that the popular oppositions of "power/resistance" or "public/private" cannot alone explain the political process (Hemmings 550). Affect was offered by its proponents as going deeper, capturing also the "qualitative experience of the social world" and embodied experience, but also offering alternative models of subject formation and interrelation (Hemmings 549-550). On the forefront among these scholars were feminists, who with their insistences on the dictum that the "personal is political" had enabled the first sustained scholarship on emotional life and later theorizations of affect (Ben Anderson, Encountering Affect 6). But affect is also a concept that can and is seen critically, as somewhat overused in contemporary scholarship for every non-representational effect or configurations of bodies. ${ }^{35}$ Still, it is a useful conceptual shorthand, a tool well-suited to capture the affective-emotional driving forces behind DIY techniques.

Lacking a comprehensive definition, affect has been used in different contexts for different purposes and with different critical purchase. Since my use here, admittedly, is a rather broad one, I will briefly summarize some of the defining, overarching characteristics of affect. As Ben Anderson rightly claims, first of we need to acknowledge that there is not one but innumerable affects (Encountering Affect 13-14): He lists some of the

33 Many examples can be found in Gregg and Seigworth's Affect Theory Reader, 2010.

34 cf. for example Hemmings 554-55, or Clough 206.

35 cf. for example Grossberg, Lawrence / interviewed by Seigworth and Gregg. 
contexts in which "affect" has been used as a descriptive term for different phenomena, ranging from "background moods such as depression, moments of intense and focused involvement such as euphoria, immediate visceral responses of shame or hate, shared atmospheres of hope or panic, eruptions of passion, lifelong dedications of love, fleeting feelings of boredom, societal moods such as anxiety or fear, neurological bodily transitions such as a feeling of aliveness, waves of feeling," amongst many else (Ben Anderson, Encountering Affect 5). As we can see, it is a diverse field in which affect is used. A common denominator, as Lawrence Grossberg points out, however, is that affect is in most cases defined as non-representational and non-intentional (193-95), looking at experiences outside of signification, meaning and conscious reactions. The turn to affect, as Anderson claims, is a "turn to consider the non-conscious or not-yet-conscious dimensions of bodily experience" (Encountering Affect 11-12). Or as Seigworth and Gregg argue, "affect" is the name given to "visceral forces beneath, alongside, or generally other than conscious knowing, vital forces beyond emotion" (1-2). In like manner, Gilles Deleuze defines affect as the unexpected autonomy of the body, as intensities which happen below consciousness (cf. Hemmings 551-53).

But affect, like plasticity, is also highly dynamic. On a very general level, Seigworth and Gregg argue, "affect is found in those intensities that pass body to body (human, non-human, part-body, and otherwise), in those resonances that circulate about, between, and sometimes stick to bodies and worlds, and in the very passages or variations between these intensities and resonances themselves" (1). Affect is found both in the intensities themselves and in their flow between "bodies." As such, affect is often less observable and more perceptible. Eve Sedgwick's idea of affects as "free radicals" (62) highlights this dynamic nature of affect: Sedgwick's starting point in her discussion is that affects, such as shame, can combine with any aspect of life: "Affects can be, and are, attached to things, people, ideas, sensations, relations, activities, ambitions, institutions, and any number of other things, including other affects." (Sedgwick et al. 19) ${ }^{36}$ As such, affect is lively, potent, but also inherently relational. Summarizing Silvan Tomkins take on affect, Hemmings writes:

Tomkins' work suggests that affects have a complex, self-referential life that gives depth to human existence through our relations with others and with ourselves. In terms of our relations with others, Tomkins asked us to think of the contagious nature of a yawn, smile or blush. It is transferred to others and doubles back, increasing its original intensity. Affect can thus be said to place the individual in a circuit of feeling and response, rather than opposition to others. (Hemmings 551-53, italics in original)

For Tomkins affect serves as a connection to others; affect is defined as relational. Similarly, Deleuze defines affect in relation as well: He prominently saw affect as potentiality, namely as "a body's capacity to affect and be affected" (cf. Gregg and Seigworth 1-2). In its relationality, affect thus is collective, extending beyond the individual.

The prospects that come with new bio-medical-technologies generate many different affects: hopes, fears, high expectations, dread. They are celebrated by some, con-

36 For more detailed discussions of Sedgwick see amongst others B. Anderson (2014) and Hemmings (2005). 
demned by others. In culture and society, biotechnologies often create anxiety, a "vague apprehension," diffuse, but immediate and unsettling (9); an uneasiness that often is displayed also in representations of biotechnology in the media, policy debates and fiction (Brodwin, "Introduction" 9-10). On the other hand, many of these representations also create a vague sense of hope and a promissory outlook on the future. Much of this is created not through the technologies themselves, the devices and tools, but through the biotech imaginaries in the public sphere: the narratives constructed around them, the speculations about future applications, the simplifications. It is not necessarily the technologies that generate affect but they spark the imagination of the viewer. Promises, hopes, and fears draw on the scientific imaginaries created through (pop)culture. These imaginaries, it seems, produce visions of utopia, by Grattan seen as a "desire or hope that the world could look better than the present" (2). In the cases under consideration here, these utopias are both a perfect and perfectible body and the promise of democratization, participation and control. Sean Austin Grattan in his 2017 work Hope Isn't Stupid renounces the prevalence of negative affect in criticism and instead wants to achieve a more complex engagement with affect that "resembles the complexity and difficulties of lived experience" (1). Looking at utopian discourses and the "affective basis for utopian desire" in contemporary American novels, he demonstrates that hope is not as naïve as criticism often labels it, but a complex site of critical inquiry. Grattan frames utopia as "the oscillation between utopian desire and the actualization of that desire" (35). The utopian discourses created by (bio-)technological imaginaries, thus, create a desire and hope for the fulfillment of their promise of perfection and malleability. More than in Grattan's narratives, the desire for the fulfillment of such promissory bodies - of bodily utopias - here includes the conscious modification of real bodies. ${ }^{37}$ But still, they show that hope, indeed, is not stupid but an inherent part of how we engage with the promises and threats of biotechnological advancements. 'Promises,' therefore, can be seen as inherently affective: they are non-representational in that they are impossible to represent, yet prominently perpetuated by representation; most of the time they are creating a non-conscious desire; they can attach to and shape almost anything (promises can emanate from and target most of what Sedgwick includes in the quote above); and most of all they are dynamic and relational, born in the intersection between bodies, institutions and technologies, in this case.

The role representations play in perpetuating promises, desire, anxiety, makes it useful to briefly focus on one of Ben Anderson's conceptualizations of affect as a "col-

37 Of course, the promissory bodies in biotech imaginaries are also full of meaning and signification. Ben Anderson emphasizes that when analyzing affect representations should not be overlooked, which tends to happen because affect is often theorized as non-representational. For Anderson, representations are part of the collective nature of affect. Despite their somewhat different theoretical conceptualization - standing on opposing poles of poststructuralism and materialism affect and representation do not preclude each other: "Instead we must pay attention to how representations function affectively and how affective life is imbued with representations." (Encountering Affect 13-14) 
lective condition" (as in "atmospheres of hope"). ${ }^{38}$ This conceptualization is particularly useful for a view of affect in a given cultural formation, especially on a collective level. Anderson defines affective atmospheres as a form of collective affects that "envelop" life. For him, affective atmospheres (such as "Obama hope") are impersonal because they belong to the collective situation, but they are felt as intensely personal. This is true for many of the affects that play a role in this book, such as anxiety, desire, hope, or dread. Affective atmospheres, according to Ben Anderson, "may interrupt, perturb and haunt" places, persons, and things; they are not static but - like plastic bodies - always in a process of becoming, "forming and deforming, appearing and disappearing" ("Affective Atmospheres" 78-80). The intangible nature of affect as employed in Anderson's concept of the atmosphere is well-suited to capture how affects shape DIY biology. They are not tangibly there but always present, located in the collective and the personal (without being distinguishable as originating in either of them). In biotechnology, I would argue, 'promise' becomes a collective condition, a unifying principle, yet also something intensely personal, intimate (as it pertains to the make-up and form of individual bodies).

These promises are wide-reaching: Promises of new biotechnologies to cure diseases and enhance your quality of life; promises of enhancement, longevity and personal development, promises for the economy and healthcare system; and promises of democratization and participation. They all shape how individuals engage with biology and biotechnologies. As such, these promises gain a certain degree of power over how people envision, shape and govern their bodies, in the name of health, the marketplace and (political) participation. Affective life, Ben Anderson writes, is "imbued with relations of power" (Encountering Affect 7-8). Nigel Thrift, originally concerned with spatial geography and affect as a vital element of cities, argues that we should see affect as political, something that can be manipulated by the powerful for political ends (58). The turn to affect, he claims, creates new political registers, affective collectives and intensities (58). The power of promise, then, is not just representational - creating new utopian imaginaries of promissory bodies - but also material and political.

As elaborated on in the previous subchapter, "[a] cultural approach opens up the body to the investigation of meanings and values, metaphors, attitudes and beliefs,..." (Pitts-Taylor, Cultural Encyclopedia xxvii). Materialist approaches - be it through theories of the body, plasticity or affect - on the other hand, convince with a much more sustained engagement with the lived materiality and reality of embodiment. The body, its texture and feeling(s), comes to be center stage. It is seen as entangled in its environments, as not just representational or symbolic but real, which paradoxically might result in a new form of biological determinism. The new focus on materiality and the material agency of things can require epistemic practices and old conceptions of power, agency and authority to be questioned (cf. Pitts-Taylor, "Mattering" 11-12). How is matter involved in the production and rehearsal of power relations? How is biology governing and being governed?

38 Ben Anderson delineates different forms of affect that require different forms of inquiry: affect as "object-target," "bodily capacity" or "collective condition" (such as "atmospheres of hope") in different situations and places (Encountering Affect 4). 


\subsection{Discourses of Power, Risk and Responsibility}

According to many wide-spread accounts of culture - prominently among them Foucault's - culture is a technology of power, a form of managing populations and societies through surveillance, discipline and modification of behavior, feelings, and thought. While I believe that power is only one, albeit crucial, dimension of culture, it cannot be denied that discourses of power, governmentality and population management have shaped and will continue to form the applications of biotechnology, also in the context of DIY biology. Many of the dominant themes of studies working in the direction of biopower and governmentality - such as the function of medicine as form social control (including the policing of difference and deviance), agency in healthcare settings, power relations, ideology, citizenship, social movements, and risk discourse - influence not just the rise of DIY techniques but how they can and will be used, for what ends and purposes: as tools of empowerment and citizenship practices, as mechanisms of responsibilization, control and subjectification.

Many of those concerns are related to Nikolas Rose's infamous notion of the new "politics of life itself." In response to concerns about new eugenics, Rose delineates a historical shift from biopolitics of populations - where the focus was on hygienics and the regulation of reproduction on the level of the nation as a whole - to ethopolitics, understood as "individual management of the 'somatic' self" (Braun 10). Following a broadly Foucauldian approach, Nikolas Rose argues that contemporary vital politics are "politics of 'life itself'." What does he mean by that? He argues that the vital politics of our time are no longer concerned merely with health or illness or population management per se, but " $[r]$ ather, it is concerned with our growing capacities to control, manage, engineer, reshape, and modulate the very vital capacities of human beings as living creatures" (Rose, Politics of Life 3-4). While his treatise of how "life itself" today is brought under the rule of governance, risk, and individual responsibility could already be considered outdated, it still remains a noteworthy influence on many contemporary thinkers. His account of ethopolitics and molecularization has become a standard narrative of the governance of bodies in (medical) culture and should therefore be mentioned here as well. This subchapter will first introduce the theoretical ideas on biopower and biopolitics from Foucault to Rose before moving on to discourses of risk, vulnerability and biocitizenship.

\section{Biopolitics Now and Then}

From the $18^{\text {th }}$ century onwards, Michel Foucault argues, the tools through which political power was exercised have shifted from decisions about life and death ("the right to take life and let live," The History of Sexuality 136) to the management of life, on the population and individual level. Politics since then addressed the vital processes of human life, such as sexuality, reproduction, birth, death, health, disease, as well as their ordering and enhancement. Foucault uses the term "biopower" to describe the rise of diverse techniques aimed at the "subjugation of bodies and the control of populations" (History 140): He differentiated between "anatomo-politics" - those techniques or "disciplines" that aimed at the optimization, disciplining, submission of (individual) bodies - and 
"biopolitics" - those that focus on the population as a whole, its health, vitality and regulation (History 140). Biopolitics, thus, dealt with daily life concerns such as morbidity, mortality, longevity and tried to address and amend them through public health education, hygienics movements and so forth (Braun 8). While on the first glance the former seems to come into effect in DIY biology and medicine - the optimization and control of individual bodies - DIY, as we will see, might also have potential for the latter: As Foucault writes, the "right" to health and happiness, the right to "rediscover what one is and all that one can be" is one (political) outcome of this bio-power aimed at the facilitation and fostering of life (History 145). This right is also instrumental in DIY biology and medicine: The right to know, to tinker, to enhance. But DIY biology and medicine also builds on the age-old fundament of biopolitical strategies of population management that Foucault and later on Rose describe, and future applications might see this side rise to more prominence.

According to Rose, the biopolitical strategies in the early $20^{\text {th }}$ century primarily tried to modify public attitudes and individual judgments (and action) through education and counselling. A good example is the public health movement in the $19^{\text {th }}$ and $20^{\text {th }}$ century, which tried to instill basic tools of hygiene into the daily life of the population through facilitating access to sanitation, social work and the tools of the new media, such as advice pamphlets. This included both maximizing population fitness by encouraging positive habits to promote physical and moral health (preventive medicine and public health education) and improving population fitness by "acting upon the reproductive decisions and capacities of individuals" (commonly called positive and negative eugenics) (Rose, "Politics" 4). Contemporary biopolitical strategies, in contrast, differ from these in that they are no longer oriented on the level of the population but individualized: Health promotion is still its main objective but today states see themselves as enabler and facilitator while the obligation for managing and monitoring health lies with the individuals themselves. For Rose, these new strategies are part of a molecular biopolitics in which a molecular style of thought (focusing on the investigation and engineering of molecular processes) intersects with broader questions of how to govern ourselves and others, with those molecular knowledges at hand ("Race, Risk" 436). Rose identifies five pathways in which mutations occur or have occurred on the way to the contemporary "politics of life itself": molecularization - life envisaged at the molecular level, understood "as a set of vital mechanisms among molecular entities that can be identified, isolated, manipulated, mobilized, recombined," echoing a mechanistic view of life; optimization - future directed interventions in the present; subjectification - new understandings of what humans are or should be, new forms of "biological citizenship"; somatic expertise - new ways of governing through new forms of pastoral power and expertise; economies of vitality - the search for biovalue, rise of bioeconomics and exploitation of biocapital, furthered by a reductionist and mechanistic view of life (Politics of Life 5-7).

The name Rose gives to this dominant biopolitical regime of the $21^{\text {st }}$ century is ethopolitics: The new "politics of life itself" goes hand in hand with an increasing responsibilization of individuals to manage their health with "a prudential eye on the future." Patients are encouraged to become consumers of medical and diagnostic services and products, actively and responsibly making choices about their body and health: 
"This complex of marketization, autonomization, and reponsibilization gives a particular character to the contemporary politics of life in advanced liberal democracies." (Rose, Politics of Life 3-4) Citizens are encouraged to take an active role in their health and well-being: In a "complex network of forces" - between enterprises capitalizing on new health strategies, state advice, private campaigning organizations and self-help groups, medical experts and health professionals - "the health-related aspirations and conduct of individuals is governed 'at a distance', by shaping the ways they understand and enact their own freedom" (Rose, "Politics" 5-7). Disciplinary power is applied through mass screenings, health risk appraisals, fitness tests, health education campaigns that evoke guilt, anxiety, and desires. The rhetoric used is such that individuals remain oblivious of its disciplinary nature and health is promoted as a right and goal for each individual (Lupton, Medicine 32). Corrective encouragements, therefore, are seen as benevolent and not controlling: "Thus, in being aware of the public gaze, the individual unconsciously him- or herself exerts disciplinary power, both over others and over the self through selfregulation," Lupton writes. In consequence, power relations are dispersed and made invisible (Lupton, Medicine 32). Such a discourse holds true for many of the techniques of DIY biology and medicine: seemingly based on choice and individual responsibility, in truth they are highly disciplinary.

What is noteworthy is that Rose delineates new modes of individualization and collectivization in this process: Individualization here occurs through the processes of responsibilization that turn health into an obligation and expectation and a personalization of the management of the (somatic) self and the body. Despite the dominant themes of such personalization - age-old ideals such as "autonomy, control, responsibility, prudence" - according to Rose we should not forget that this individualization takes place in relation to a collectivity, a biosocial community, and its norms and expectations ("Race, Risk" 436-37). Those parallel processes of individualization and collectivization are interesting because they mirror those prevalent in DIY biology: an individualization of responsibility and a personalization of technologies, coupled with a somewhat abstract sense of collectivization (complete with new social formations and forms of peer pressure) and comparison to a an even more abstract set of data.

\section{Techniques of the Self, Techniques of the Body}

The self-normalizing techniques that are so prevalent in the contemporary politics of life are called "techniques of the self" by Foucault: "[T]echniques which permit individuals to effect, by their own means, a certain number of operations on their own bodies, on their own souls, on their own thoughts, on their own conduct, and this in a manner so as to transform themselves, modify themselves, and to attain a certain state of perfection, of happiness, of purity, of supernatural power, and so on" ("About the Beginning" 203). ${ }^{39}$ Abend and Fuchs, following Mark Butler's narrative, recount the history of Foucault's techniques of the self from antiquity to modernity and postmodernity. In antiquity, techniques of the self included inward oriented, "care-taking" techniques 
that the self exercised on the self, such as meditation, examination of conscience, mental representations, or most prominently verbal confession. In modernity, according to M. Butler, economically driven work on the self for productive self-engineering was the most popular form of techniques of the self, while postmodernity is characterized by a playful interaction with notions of the self and identity. Abend and Fuchs argue that in the contemporary moment, techniques such as the Quantified Self merge all these aspects - care-taking, work, play - in their technologies, thus combining many of the previous "techniques of the self" (Abend and Fuchs 9). This is certainly also true for many other DIY techniques under consideration here.

Still, Abend and Fuchs see a transformation in the original concept of the techniques of the self, a shift from subject-centered techniques to use of digital technology, from introspection to continuous monitoring and feedback (Abend and Fuchs 12-13). With the somatization of the self, this shift, furthermore, is accompanied by a change from techniques of the self focusing on the inner world of an individual to techniques that pertain to the outside, a "shift away from the examination of the consciousness and soul towards the examination of the body" (Abend and Fuchs 12). Marcel Mauss described such techniques as "techniques of the body:" From an anthropological perspective, Mauss examines how (useful) everyday activities such as walking, running or sleeping are contextualized, learned and taught - how the body is adjusted to its purpose in a social setting. Mauss thereby emphasizes the intersection of the biological and social as a key feature in how bodies become a set of "techniques" (Thacker 56; Abend and Fuchs 12).

\section{Risk, Vulnerability and Responsibility}

As the discussion of Foucault and his disciples will have illustrated, contemporary molecular biopolitics takes on a new quality of uncertainty, (un)predictability, and vulnerability. Vulnerability, here, can be considered as an overarching concern - one that is also inherently related to new materialism - which is why I will first introduce its most relevant characteristics, before relating them to the discourses of risk, susceptibility and responsibility. Broadly two strands of conceptualizations in scholarship on vulnerability can be discerned: One approach sees vulnerability as the "ontological condition of our humanity:" "[t]o be vulnerable is to be fragile, to be susceptible to wounding and to suffering," a condition that is a shared feature of human embodiment. In this view, often, human life, the human body is vulnerable in itself but also in relation to others, dependent on their actions, care or support "to varying degrees at various points in our lives" (Mackenzie et al. 4-6). According to Judith Butler, for example, the human body is inherently vulnerable to the actions of others, with some parts of the population being more exposed, more precarious than others. ${ }^{40}$ Other definitions of vulnerability focus more closely on the susceptibility of particular groups

40 cf. J. Butler Precarious Life. In their 2016 essay collection, Vulnerability in Resistance, J. Butler et al., moreover, look at the dichotomy between vulnerability and resistance, proposing strategies of resistance and subversion that also explicitly include questions of vulnerability and vulnerable populations. 
or persons - "those with reduced capacity, power, or control to protect their interests relative to other agents" - "inequalities of power, dependency, capacity, or need" that leave them more vulnerable to harms, threats and exploitation by others (Mackenzie et al. 4-6). Combining these two conceptualizations, Mackenzie et al. suggest a taxonomy of vulnerability that distinguishes different sources and states of vulnerability, allowing us to acknowledge both the inherent vulnerability of the human condition and the identification of contexts and situations that leave different persons or groups of persons more vulnerable than others (7-9). ${ }^{41}$ Also in the analysis and discussions in this book vulnerability as the inherent condition of human embodiment and the more context-specific forms of vulnerability will play a role: The former in relation to the discourse of risk, the desire to know more and to control the body to obliterate such feelings of vulnerability, the susceptibility to inside and outside forces, as well as the promises and fears that exacerbate its experience; the latter when we consider differently distributed access, power relations, capabilities, also on a global scale.

Much of this perceived vulnerability, we could argue, also stems from the discourses of risk so prevalent in contemporary life. Beginning in the $19^{\text {th }}$ century, the body came to be charted, calculated, expressed through tables, norms and means that gave rise to the notion of life itself as calculable and thus as assessable in risks, probabilities, and likelihoods. The complex term "risk" originally had many different, rather neutral meanings, most prevalent among them the mathematical idea of expressing the likelihood that an event may occur. While risks as pure numerical data are still rather neutral - based on studies we can calculate the probability of events - what we do with these data in our processes of meaning making, our discourses, is a different thing. Risk has received negative associations, is connoted with danger also in public health discourses: being "at high risk" means being "in danger of," for example, developing a disease (Lupton, "Risk" 460-61). In the discourse of public health, risk is often conceptualized as either external - for example environmental factors over which an individual has little to no control - or as internal - as the consequence of lifestyle choices that individuals can (and should) control (Lupton, "Risk" 461-62). ${ }^{42}$ It is this latter form of risk that

41 They differentiate different sources of vulnerability: inherent (the intrinsic vulnerability of the human condition), situational (context specific sources, potentially caused by different "personal, social, political, economic or environmental situations of individuals or social groups," short term, intermittent, or enduring), and pathogenic (created for example through "dysfunctional or abusive interpersonal and social relationships and sociopolitical oppression or injustice"). Inherent and situational vulnerability, moreover, can have different states: they can be dispositional or occurrent, potential vulnerabilities versus actually occurring ones. A quote from Mackenzie et al. will illustrate this distinction: "For example, all fertile women of childbearing age are dispositionally vulnerable to life-threatening complications in childbirth. But whether or not a pregnant woman is occurrently vulnerable to such complications will depend on a range of factors, both inherent and situational, such as her physical health, medical history, socioeconomic status, geographical location, access to health care, and cultural norms relating to pregnancy and childbirth. The dispositional-occurrent distinction serves to distinguish vulnerabilities that are not yet or not likely to become sources of harm from those that require immediate action to limit harm" (Mackenzie et al. 7-9).

42 One of the most famous evocations of such a "risk society" is that of sociologist Ulrich Beck. Beck looks at the risk society as a culture increasingly aware of its potential for destruction through 
has gained a lot of cultural resonance in late-capitalist society: Here, the individual is seen as responsible for avoiding health risks for their own wellbeing but also the good of society (by, for example, preventing illness to lower health care expenditure) (Lupton, "Risk" 463). As such risk is also a constructed, normative discourse that aims at governing bodies and lifestyles. It is predominant in many of the contemporary discourses around health, for example when it comes to smoking, drug and pharmaceuticals abuse, physical fitness and obesity, overeating and diabetes, even heart disease. This risk discourse works through "calculations about probable futures" followed by "interventions into the present" (7) in order to control that future, a logics of prevention and preemption: Identifying those deemed at risk (individually and collectively) and then acting on them through observation, treatment, responsibilization and (self-)management (Rose, Politics of Life 7, 70-71, 107). Risks, it has been proclaimed, are to be avoided and reduced. ${ }^{43}$

When risk is communicated to individuals (and collectives, for that matter), for example through individual test results or in publications, risk factors are often framed as susceptibilities to certain diseases or conditions. An offspring of earlier theories of "predisposition" and inheritance, the molecularization of risk and biological information moved susceptibility from correlations of different factors - such as age, weight, and family history - to the molecular make-up of individual bodies (Rose, Politics of Life 18-20; 84-87). In order to describe the rise of genetic risk, or genetic susceptibility, one needs to look closer at how genes and disease are connected: In contrast to the expectation of the Human Genome Project (to find clear genetic causes for disease by offering a 'genetic roadmap' of sorts), its completion led to the realization that only a small number of human diseases are caused by a single gene (Huntington's disease being one of those mono-genetic diseases). Many more conditions are associated with changes in single nucleotide bases at different locations in the genome: Those Single Nucleotide Polymorphisms (SNPs) contribute to the risk of developing conditions like heart disease, diabetes, or cancer. ${ }^{44}$ Their discovery and study, thus, contributed a great deal to the implementation of a logics of probability and susceptibility, by putting in numbers or relations the likelihood of developing a certain condition. This form of thinking along the lines of SNPs opens genetics up to the discourse of risk assessment, prediction and

nuclear war, environmental and biological catastrophes (Tomes 625). According to Beck, risk is an intrinsic part of the experience of modernity, calculated in numbers and probabilities and known through science. He argues that the risk society focuses on the distribution of risk, not wealth, among the population. This distribution of risks inverses the distribution of wealth: wealth accumulates at the top, risk accumulates on the bottom - but in the risk society no one can fully escape risks anymore, even though the wealthy have more means to circumvent them (Lidskog).

43 This becomes problematic when we can no longer live with risks. "Risk literacy" is a skill-set used to set risks into context (also with each other) and, based on reliable data, make informed choices about the risks we are willing to take. For risk literacy in medical decision making, cf. Operskalski and Barbey.

44 Genetic tests, then, scan for such SNPs and calculate a numerical risk of contracting a disease, adding or subtracting the risks associated with single SNPs often on multiple loci on multiple chromosomes to form an overall overview. While the SNP itself does not have to be the culprit, it does indicate that something in the vicinity may lead to a risk not experienced by somebody who does not have that specific SNP. 
management (cf. Rose, Politics of Life 18-20; 84-87). The "premonitory knowledges" (Lock) - knowledges that "bring potential futures into the vital present and make them calculable" - create obligations for the self to act in the present upon the self, one's body, one's health, one's choices with an eye to that potential future (Rose, "Genomic Susceptibility" 147).

This connection between screening and (early) intervention is an intrinsic part of the politics of life in the 21st century and seems to proliferate with the development of sophisticated testing, screening, and imaging technologies (Rose, Politics of Life 18-20; 84-87). Today, individuals can be tested for a growing number of single-gene disorders and also many SNP-based tests are available to determine the likelihood of developing a disorder and many of those tests are not just self-chosen but also self-administered in a DIY fashion. ${ }^{45}$ Susceptibility, then, describes a certain vulnerability, a personalized form of risk. The "molecular politics of (genetic) risk" that Lemke formulates following Foucault's bio- and anatomo-politics transcends concerns with discipline and discrimination and aims at design, at configuring "healthy" human existence ("Disposition" 562). Lemke argues that the concept of information is crucial: "In the molecular genetic perspective, information at the same time serves as the 'code of life' and as the 'key to freedom" (558). The assumption is that with sufficient information, risk management and lifestyle changes, illness can be avoided. ${ }^{46}$ In Lemke's position, we can see how prudent decisions are possible only when individuals have enough medical and genetic knowledge about the self ("Disposition" 558-59). This connection between the possession of information and prudence in the face of ever-present risk (factors) also demonstrates how risk can become a critical factor in many self-directed interventions into the body, especially those who aim at a deeper knowledge of one's own biology.

Genetic governmentality, Lemke argues, creates a new individualized body politic, "which calls on us to be as economic as possible with our own body, health or 'quality of life'," but also gives rise to new forms of (collective) social identity, political activism, and "biosociality," to use Rabinow's term ("Disposition" 561). It creates new forms of vulnerability and new ways of assessing and defining the self in relation to its biology. Risk, thus, manifests in individual bodies and lives, but also in the assemblage of bodies and collectives. ${ }^{47}$ The high prevalence of this form of risk thinking generates a sense that all persons "though existentially healthy are actually asymptomatically or pre-symptomatically ill" (Rose, Politics of Life 18-20; 84-87) and thus gives rise to new

Genetic and molecular tests as a type of DIY biology and medicine are analyzed in Chapter 8 . just for biological risks, though they require more specialized biological knowledge, but also day to day risks and probabilities, such as the probability of winning the lottery or the risks associated with flying or other forms of transportation.

47 Another interesting means of conceptualizing bodies at risk in a globalized society is seeing them as being part of a global (molecular) economy of exchange and circulation, as being part of a molecular world filled with risks: of infection, contamination, and so forth. This type of risk is expressed in relation to global or transnational pandemics (such as bird flu, Ebola, the Zika Virus). Braun more closely looks at this understanding of the molecular body as displaced in wider molecular fields of risk (Braun 7). 
subjectivities. Risk is increasingly treated "as if it were an illness in and of itself:" More screening and more monitoring of bodies and bodily functions leads to an increasing medicalization (Conrad, Medicalization 163) and creates new categories of individuals, "new individual and collective subjectifications of those 'at risk" (Rose, Politics of Life 19). According to Rose, the person "genetically at risk" is born at the intersection of at least three trajectories in contemporary bio-medical practice and thought: the growing belief in society in a genetic basis of many conditions (both as mono-genetic diseases with clear causation and in the form of susceptibilities), the confidence and ability on the part of researchers to find genetic markers associated with a condition, and the claims of doctors to identify conditions through diagnostic tests (Politics of Life 106-07). The fear connected to the increasingly subjectification of people as "persons at risk" is that they will be treated differently in the work market, with regard to health insurance, or in their personal life, even though many contingencies remain and the outlook is only probabilistic (the penetrance of a gene may be unknown, time and severity of unset uncertain) (Rose, Politics of Life 70-71, 107). I would argue that it is also this fear of biological determinism, of being defined via one's genetic biology, that gives genetic testing an aura of certainty that it does not (yet) have. The "promise of certainty" attached to genetic testing is only illusory (Rose, "Politics" 11-12): Too many factors influence the actual development of the disorder, even in cases such as Huntington's - previously thought to be a certainty - where the length of the wrongfully added nucleotide sequence now is believed to have an impact on the time of onset and severity of the disorder. Genetic testing, then, opens another space of uncertainty populated by those "asymptomatically or presymptomatically ill," those who carry the markers but are not (yet) sick (Rose, "Politics" 11-12). Similarly, the choice to act or not act in response to knowledge about risks and susceptibilities is only illusory (Lupton, "Risk" 466). As responsible citizen, the somatic self is obliged to act or suffer the consequences of moral isolation, social stigma and sanctions.

Neoliberal governmentality needs and fosters subjects that understand that they are responsible for themselves and act accordingly. ${ }^{48}$ In neoliberal democracies, thus, individuals are obligated to seek out information (about the self), take action, maximize their quality of life and life chances - to act responsibly towards themselves, others and society at large (Rose, Politics of Life 107-08). In public health discussions, the discourse of "at risk" allows us to judge and moralize the behavior of individuals, assigning guilt and responsibility: genetic explanations thus do not lead to responsibility being taken from the subject, but the subject rather gains a new "genetic responsibility" (Lemke, "Disposition" 558-59). This genetic responsibility does not just change how one should act prudently for oneself, but genetic illnesses are thought of in familial terms as affecting past, present and future families and thus create new obligations to one's partner, children, career or other financial obligations (Rose, Politics of Life 107-08). As we can see both in Rose and Lemke's account, health has become a moral responsibility and a personal duty, to oneself and others. This return of moralizing discourses, however,

48 For more on this see for example Mitchell Dean, Governmentality: Power and Rule in Modern Society, 1999. 
happens stealthily, 'through the backdoor:' It is no longer bound on religious precepts but on scientific norms and a covertly disciplinary politics of life.

\section{Biological and Scientific Citizenship}

According to Rose, the subjectification of individuals under new forms of (self-)government of body and self, leads to a new form of "biological citizenship." He uses "biological citizenship" descriptively for all projects that "linked their conceptions of citizens to beliefs about the biological existence of human beings," as individuals, men, women, families, linkages, communities, populations, races, or species (Politics of Life $132-34 ; 140-41 ; 149 ; 223)$. Rose and Novas describe "biological citizenship" as both individualizing and collectivizing: Individuals relate to themselves with knowledge of their somatic individuality and concomitant responsibilities and are embedded into new forms of "biosociality" and collectivities around biomedical classifications, online and offline (Rose and Novas 441-42). ${ }^{49}$ Similarly, Torsten Heinemann writes that biological citizenship acknowledges the role of new bio-social collectivities - such as patient advocacy or self-help groups - for new forms of public participation, patient empowerment, and involvement in political debates. ${ }^{50}$

Biological citizenship requires those invested in their biology to become political: It involves "individuals and groups claiming their rights and struggling in the micro politics of health and in the macro politics of health care systems for funding, for research, and for provision" (Rose, "Genomic Susceptibility" 147-48). The requirements of Western neoliberal democracies to be flexible, to pursue life-long learning, to be perpetually assessed, to be nudged to improve oneself by monitoring one's health and managing one's risks, means that active, responsible biological citizens engage in self-evaluation and continuous modulation (Rose, Politics of Life 154). Biological citizenship, therefore, follows an ethics of activity, "in which the maximization of lifestyle, potential, health, and quality of life has become almost obligatory," whereas those who do not comply, who do not "adopt an active, informed, positive, and prudent relation to the future" are negatively judged and sanctioned - not by formal, government-directed power but by peer-pressure and comparison (Rose, Politics of Life 24-25). Biological citizenship, thus,

49 Paul Rabinow's concept of "biosociality", suggests "the emergence of such new social communities around particular biological conditions" and describes a "shift from a socio-biological culture which is modeled on nature to the culturalization and engineering of nature itself" (Burri and Dumit , "Introduction" 5). For Rose and Novas the internet is crucial as a means of communication and information exchange - literacy is a key term here - and as a community-formation device. For them webpages and virtual communities perpetuate their ideal of active, biological citizenship in that they encourage people to become involved in communities of people that define themselves along biological, often molecular or genetic, lines and thus to become politically active in a new way (Rose and Novas 445-46; Rose, Politics of Life 145-46).

50 Influential examples of such bio-social collectivities are patient advocacy groups, such as the Michael ]. Fox Foundation for Parkinson's Research. Like them, some of these foundations and advocacy groups also contribute to clinical research on the progression of diseases, patterns and clinical markers - providing their members with new possibilities to contribute to the research of their diseases and in a second step to the clinical testing and development of new medications. 
does not only consist of political claims to rights and provisions, but also includes a specific form of care for the self (Rose, "Genomic Susceptibility" 147-48).

This form of biological citizenship is linked to what Rose terms "scientific citizenship." Today, the sweeping advances in scientific and technological progress have led to the increasing need to educate citizens so that they can engage in informed debates over the resulting complex ethical and democratic dilemmas. Fostering the "public understanding of science" for Rose and Novas has two core functions: For one, it is a means to both address and compensate the "democratic deficit" said to exist when citizens are not active participants in the formation of scientific and technological futures. Moreover, a better understanding of science can also help to regain the trust and confidence of lay persons in the regulatory mechanisms of science (Rose, Politics of Life 140-42; Rose and Novas 445-446). It is this mindset that builds the foundation for arguments for "science education for citizenship" (Kolstø). Scientific literacy, here, is seen as a skill that all responsible citizens should have. The result of this entanglement of scientific education and decision-making process is a new form of scientific citizenship.

"Scientific citizenship," then, requires individuals to take an active role in enhancing their own scientific and biomedical literacy, to actively and dynamically search out knowledge - a process made comparatively easy through the internet as a powerful way to access information and exchange ideas (Rose, Politics of Life 140-42). Scientific literacy and citizenship go hand in hand: People need to be educated about biotechnologies and their implications in order to make decisions about their value and use. They need to be educated about positive and negative impacts of technologies to counter mistrust and foster open communication. This type of scientific literacy, however, is often not facilitated. Rather, contemporary biopolitical strategies create an obligation and compulsion to self-educate: responsible choices can only be made on the basis of knowledge. It is this form of active biological and scientific citizenship that is required and espoused by the "techniques of the self" under investigation in this book. DIY biology and medicine requires a high level of citizen engagement in science, scientific literacy, and a selfdirected curiosity about the biological basis of life. Its techniques subject their users to this new form of self-management. DIY biology and medicine is political, exactly because it is based, to a large degree, on the older structures of risk assignment, population management, responsibilization and citizenship described in this subchapter.

\subsection{Coda: Why Representation Still Matters}

Because they are embedded in diverse trajectories and politico-economic logics, the "politics of hacking life" are complex and multifaceted (Meyer, "Hacking"). To gauge them in their full complexity, many of the tools and theoretical resources developed in this chapter will surface again and again in this book. As I have shown in the discussions above, different approaches to the body and embodiment create new points of view and new conceptualizations, but also new problems. They bring with them both advantages and disadvantages. Both constructivist and materialist approaches fail in one way or the other in their pursuit to adequately theorize and capture the lived real- 
ity of bodies. Constructivist and poststructuralist approaches risk denying the material reality of life, of embodiment - such as the material reality of pain felt in and through a body. They cannot fully address what is at stake in contemporary biopolitics, especially when it comes to the material transformations of bodies through capitalism and power relations (Pitts-Taylor, "Mattering" 7). At the same time, however, materialist approaches risk denying the importance of culture for how bodies are lived, experienced and shaped. By failing to include representation into their discussions, they deny its material effects on bodies: how they shift and change in response to representations, how individuals decide to transform their bodies according to their preferred symbolic meaning.

The question that arises, then, is how to reconciliate the insights of constructionism with the need to attend to matter, the body, materialism, ontology? In order to redress the shortcomings of these lines of thought in isolation, newer approaches try their hand at a combination of materiality and governmentality, of materiality and symbolic representation, of ontology and epistemology. Disability studies seems to be a promising beginning for this exploration: While disability theorists have always insisted on the socially constructed nature of disability, they asserted its material reality as well..$^{51}$ Tobin Siebers, for example, tries to capture this contradictory experience in his notion of complex embodiment. Siebers uses this term to describe the experience of having a "materialsemiotic body," a body that is material but also symbolically shaped. This theory sees the body and representation as mutually transformative: "Social representations obviously affect the experience of the body...but the body possesses the ability to determine its social representation as well" (25-26). Donna Haraway uses the idea of the "materialist-semiotic" to combine bodily materiality with the discursive-linguistic to obliterate the divide between nature and culture ("Situated Knowledges"). More recently, Victoria Pitts-Taylor applies such a material-semiotic, an ontologist and epistemologist, view in her discussion of the plastic brain in order to understand the "making and meaning" of bodies, "without denying them realness" (Brain's, 1). Such approaches are useful in a cultural study of bodies because they stress the need to include representations into considerations of the body: Representations can contribute a great deal to how people experience and value their bodies, how they want to transform them, what meanings they read into them.

In such materialist-semiotic approaches, we encounter the body as indeed being formed by two highly intertwined entities: one semiotic body, one material or physical one, with the latter being turned into the former in and through representation. This abstraction is a conscious choice - as we know signs are never completely equivalent to their signifier - about what is represented. The semiotic body of representation, thus, will only ever capture parts of the lived reality of the material one, while the material one, in turn, is shaped by the abstractions and simplifications inherent in the

51 Many theorists in disability studies argue that bodies are 'disabled' through culture, society and their environments, for example when buildings are not designed as or converted to be accessible: It is not necessarily the body that is 'disabled' but the lack of a ramp instead of a staircase that 'disables' the body. At the same time, they do not want to negate the lived experience and reality of pain and physical limitations. 
semiotic process. To give a more practical example, human fantasies of perfection in science fiction focus in their representations on the positive sides of enhancements the promise of superiority, of infallibility, of physical extension and (in some cases) immortality - while they often choose to ignore the negative sides, the pain, the trauma, the isolation that potentially comes with material transformation. What we see, thus, is, again, a utopian vision. But it is this utopian vision that, to follow Wegenstein, in a circular gaze shapes the expectations and experience of the material bodies in front of the screen (Cosmetic Gaze 2). ${ }^{52}$ This is also how representation, the imaginary sphere of biology, still 'matters' in the most literal sense. ${ }^{53}$

In the contemporary DIY techniques and technologies, the body is not (only) seen as a social construction, but indeed as a material reality, an assemblage of pieces. The engagement in social and ideological contests over the body, its meaning and significance, makes them decisively political. They are part of the new politics of life, new forms of individual and collective subjectification in which our "biology" is "highly salient to practices of identity formation" (Rose, "Race, Risk" 430). However, while identities can be constructed in and through DIY techniques, the body is the tool to work with and at the same time the material to be worked on. In DIY techniques, nature and culture intersect and are of equal importance. In my analyses, therefore, I choose a combined approach via representation and its material effects on bodies. Representations, visual and linguistic ones, are understood as evoking and transmitting affects between and beyond bodies. These affects materialize in bodies, determining how individuals experience, live in and judge their bodies, take action to reform and shape them. ${ }^{54}$ What results is a "corporeal politics of plasticity" (Pitts-Taylor, Brain's 6), in which the body is seen as open, amenable to, even inviting transformation - a politics in which transformation is indeed a cultural requirement. This is also an effect of the insertion of affective life into neoliberal pursuits of re-shifting market relations to "life itself," here in the form of subsuming more aspects of daily life into the dictate of the market, for example by creating bodies that conform to ideals of fitness or heralding self-optimization in the name of the market. ${ }^{55}$ In DIY technologies, therefore, we find complex entanglements of representation, power, capital and identity with the material body.

52 Social Media, especially visual media like Instagram, can be considered as an even more up-to-date medium through which such a circular gaze is created. In connection to disease, an overarching question resulting from this preoccupation with utopian visions of the body is whether or not some co-morbidities of disease states (e.g., depression) might be linked to the social-and emotional pressure to conform to desired body images.

53 This duality of meaning is definitively intended: Representation is still important - despite a lack of engagement with the sphere of the representational in new materialism - but representation also has a high influence on 'matter.'

54 Latham and Derek P. McCormack, for example, argue that representations function affectively (Thinking with Images, 2009). Similarly, Ben Anderson claims that a focus on materiality does not exclude representation when he writes: "Attention to affect does not preclude an attention to representation and affect is not somehow the non-representational 'object' per se. Instead, we must pay attention to how representations function affectively and how affective life is imbued with representations." (Encountering Affect 13-14).

55 Broadly following Anthony Negri's writings on the "real subsumption of life” Ben Anderson argues that in "real subsumption of life" desires, subjectivities, and needs mutate with capital circulation 
How do the politics of life created by DIY biology materialize in bodies and corporeal processes? (Popular) culture offers ample examples of how individual bodies and our experience of and with (our) biology are transformed through DIY practices. From these we can infer the underlying values and assumptions about what it means to be biological, to be human, to have a body, about what is accepted and what is policed. Acknowledging the inextricable entanglement of biological and socio-cultural thinking means that we need to make sure that these transformations in how we see and conceptualize ourselves as biological beings prevent rather than exacerbate social injustices, discrimination and social stratification (cf. Rose, "Race, Risk" 436). 

PART II: BACKGROUND 



\title{
3 The Promise of Small Things - The Cultural Presence of (Molecular) Biology
}

\author{
"As I write this, organisms endowed with \\ genomes are learning to change the heri- \\ table features of organisms endowed with \\ genomes." (Mukherjee, The Gene, 12)
}

And this process, Siddhartha Mukherjee writes, is astonishing. Today we can "read" human genomes and have made incredible progress in our abilities to "write" genetic code. The convergence of these two is "like a headlong sprint into an abyss," Mukherjee claims: "Once we can understand the nature of fate encoded by individual genomes (even if we can predict this in likelihoods rather than in certainties) and once we acquire the technology to intentionally change these likelihoods (even if these technologies are inefficient and cumbersome) our future is fundamentally changed." (Mukherjee 12) This abyss is scientific - we do not yet know the material consequences of genetic engineering of human organisms - as much as cultural - repercussions for questions of identity, community, difference, discrimination and future development remain subject of speculation and imagination. The certainty with which (some) scientists claim that in only a matter of years we will be able to make fundamental alterations to the human genome, incites dread in some and desire in others. It is a threat as much as a promise. And genetics is only the most prominent, and far-reaching, example. The focus on ever smaller explanatory factors in biology not only brought to light minute molecular structures but also opened them up for interventions, interferences in their structures, and with them new possibilities for treatment, cure, or enhancement, new promises of health, healing, and perfection. The 'promise of small things' that inspired this chapter, thus, is a new authority over one's own individual biological make-up and the inner workings of the body, its perpetual becoming, its project-character, amenable for individualization, optimization and transformation.

This promise, however, is not anchored in the material body itself. Rather, it is created in how scientific findings are communicated to the public by scientists, how they are reproduced in media discussions, how they are inserted into the narratives of daily life, debated in private and public. In short, they are made in and through culture. Biology and its findings today are omnipresent in TV shows, movies, newspapers, ad- 
vertisements, fiction and non-fiction writing, daily conversations and practical applications. Like culture, biology belongs to the forces that shape everyday life, are immersed in almost everything we do. These bio-cultural discourses mean that (molecular) biology can only exist within a system of cultural values and practices. According to Nelkin and Lindee, the popular imaginary allows us, as critics and affected individuals, to gain access to many social concerns about and common understandings of contemporary concepts in molecular biology: "The narratives of mass culture give shape to what is seen in the world. They define what seems to be a problem, and what promises solutions; what we take for granted, and what we question" (198). The representations of biology in culture, especially in mass and popular culture due to their ubiquitous presence and high impact, create an imaginary around biology that shapes how science is practiced as much as its understanding in culture. This imaginary is even more transformative when affective networks or promissory discourses are evoked. Hype, fear, promise, anxiety determine how individuals and communities approach scientific breakthroughs, what meaning they make of them and how far they integrate them voluntarily into their daily lives. The cultural presence of (molecular) biology facilitates platforms for discussion, disperses knowledge, contributes to the pervasiveness of biomedical solutions, routinizes our engagement with biology so that it becomes completely normal to think about yourself in molecular terms. It is a symptom as much as a cause of the fascination humans exhibit with the mysteries of life, the mysteries of their own body, the mysteries of science, the mysteries of what defines them.

This chapter engages with biology as a (pop)cultural phenomenon, in the form of theory and via practical examples. As a foundation, I look at the molecularization of life and its repercussions for our understanding of the self. I then consider the semiotic qualities of molecules, how they signify more than their mere biological function. In a last step, I illustrate that the cultural presence of molecular biology and biotechnologies also resources (utopian and dystopian) fantasies of perfected bodies and creates pervasive discourses that influence how humans think about their biologies, turning them from certainties into possibilities, and lastly into 'projects' in the most literal sense making them a central component also of DIY biology and medicine. Since I use contemporary cultural discourses as a basis to exhibit that culture also has material effects, my examples in this chapter primarily come from the realm of (science) fiction, focusing on culture before moving on to material practices in the next chapters.

\subsection{Imagining Life on a Molecular Scale}

Let me start this subchapter by elaborating a bit on its title: imagining life on a molecular scale. This title combines two strands of thought that have influenced the public perception of biology (and science more generally) and that therefore are also particularly relevant for my argument . First, it hints at the change in scale - from what can be seen with the naked eye to those particles of life in the microscopic and sub-microscopic regions - that took place during the $20^{\text {th }}$ century and that I will elaborate on in the next paragraphs. But it also, secondly, ties all that to one of the processes that has particular purchase on the public perception of biology: the imagination. What we can imagine 
becomes possible, realizable, a mental picture in our mind that somehow seems graspable. Because they seem invisible, the biological imaginary around molecules gives them shape, meaning, and function. But there is also a certain uncertainty that goes with imagining, a connotation of the un-scientific, a lack of facts compensated by fantasy. And this, to some extent, is true: While we know a lot about how life 'works' on the molecular scale, we do not know all. Much of what we perceive as true is based on hypotheses, or imaginations of how it might work, that seemed to prove true through testing. But, as the history of science shows, hypotheses, even though deemed true at the time of their fashioning, can turn out to be completely wrong. This ambiguity of the imaginary - its vital potentiality and latent uncertainty - is what carries our (lay) understanding of science. Imagining life on a molecular scale gives molecules a form and a function, life is turned into a series of agentic particles and narrative connections. Let me now try to shortly recount that dominating hypothesis of our time, the narrative of molecularization.

Discourse around the body in (popular) science and culture has, as Bernadette Wegenstein calls it, increasingly gotten "under the skin:" The inner body, organs, tissue, cells, blood, she says, have quite literally been brought to light. It was fragmented into ever smaller pieces (Getting 79). This process of molecularization is one of the five pathways in which "mutations" have occurred, that Rose identified in his account of "ethopolitics" and which he describes as having a transformative impact on contemporary biopolitics. According to Rose, molecularization, simply put, can be summarized as a "change in scale" (Politics of Life 4). For a long time, the body was imagined at the "molar" level, the level of the tangible and visible, a functionally connected system of tissues enclosed by skin. It was this molar body that was examined and acted upon through the clinical gaze of the $19^{\text {th }}$ and $20^{\text {th }}$ century medicine. Today, this molar, clinical gaze has been replaced by a "molecular gaze." Life processes are imagined, understood and engineered on a molecular scale (Rose, Politics of Life 4, 11-15). ${ }^{1}$

This movement to minute details was facilitated by three streams of thought at the beginning of the $20^{\text {th }}$ century: atomism or reductionism as the mentality of the time, the scientific method of the Enlightenment that dominated the processes and research protocols of scientists and, lastly, $20^{\text {th }}$ century structuralism, shaping which types of questions were asked and what kinds of answers presumed (cf. also Roof 64-65). The most characteristic and important for the topic of "molecularization," perhaps, is reductionism: It was, Roof writes, presumed that "[s]mall, primary elements represent a locus of knowledge - of having gotten to the bottom of things" (33). Because we tend to think about the world from the primary to the more complex, finding these minute particles - "the smallest, most basic, primary, and indivisible element" - was regarded as finally providing ultimate answers for both the beginning and the end of larger sys- 
tems and processes, such as "the origin and essence of life itself" (Roof 33). ${ }^{2}$ The belief was that what could be achieved in physics, with atoms, electrons, protons and so forth, could also be achievable in biology: explaining life on physical, chemical and molecular levels (Stevens 36). ${ }^{3}$

This process, of course, had already started in medicine with the invention of the microscope and the advent of germ theory. In the $17^{\text {th }}$ century, following a mechanistic view of life, the microscope allowed for the fragmentation of bodily functions into smaller and smaller parts (Roof 36), while the broad-scale acceptance of germ theory in the $19^{\text {th }}$ century brought germs and bacteria to the forefront as causes for many common diseases. But with the beginning of the $20^{\text {th }}$ century reductionist research on the molecular level began to flourish more and more, first with a focus on vitamins and hormones, later with a renewed interest in heredity and reproduction (Stevens 29). Beginning with James Watson and Francis Crick's discovery of the structure of DNA in the 1950 - the famous double helix - towards the race to 'crack the code,' culminating in the international efforts of the Human Genome Project (HGP) from 1990-2003, DNA has dominated scientific thought and public imagination like no other biomedical finding during the $20^{\text {th }}$ century, the "century of the gene" as many critics have named it. Because of its high influence and cultural purchase, the genome is the prime example of reductionism and molecular thought. DNA, as Judith Roof maintains, can be seen as the "anticipated emblem of the era that defined it and that it defined." DNA, more than any other molecule, shows a strong correlation between what was looked for and the molecule that was found. It fit perfectly into what was being presumed in biology and reductionist thought (Roof 3 ).

Molecularization as the dominating "style of thought" in contemporary biomedicine, according to Rose, is not just an epistemological shift - a "reorganization of the gaze of the life sciences" - but also a transformation that has allowed for, even encouraged, new technological interventions into the body: knowing and transforming, discovery and application, claims Rose, are intrinsically connected in this molecular age. ${ }^{4}$ These new molecular elements that are the focus of contemporary biopolitics, Rose argues, are characterized by a new, delocalized mobility: they can be isolated, controlled, manipulated, transferred between places and organisms, and combined into previously nonexistent processes (Rose, Politics of Life 11-15). In this thought model, molecular elements are highly mobile and seemingly infinitively manipulable, so that the potentiality of human life is inextricably linked to its molecular make-up.

While Rose's account has become standard and is generally reviewed favorably, it does offer some ground for critique. In response to Rose, B. Braun, for example, claims that Rose's argument - and I agree with him there - is rather simplistic and limited

2 Music, it seems, offers itself as a suitable parallel: In music, we move from individual notes to more complex compositions - the minute parts of a piece of music determine the sound and feel of the whole system.

3 A famous book that follows this approach and that was of immense influence on James Watson and Francis Crick's discovery of the structure of DNA is Schrödinger's What is Life? (1944).

4 Examples include gene cutting and splicing or polymerase chain reaction to multiply DNA fragments. Both were theoretical hypotheses that in the process of their discovery already invited practical applications, cf. Rose ("Politics" 14-15). 
when it comes to how the body was molecularized. Rose seems to derive much of his argument from a concern with genetics and the management and improvement of (individual) bodily materiality. Braun tries to supplement this inward-directed view by arguing that we need to view molecularization as a much broader epistemological shift that does not only concern our view of the internal make-up of human bodies but also their embeddedness, or what Braun calls "displacement," in much larger networks of molecular exchange. Under the rational of "biosecurity" and biomobility, the molecular body, Braun says, is understood as "embedded in a chaotic and unpredictable molecular world" of molecular exchange and circulation, haunted by unspecifiable risks (Braun 14). The body is still molecularized, but in a different way. ${ }^{5}$ What do these differing but to a degree complementary accounts of molecularization tell us? We need to acknowledge that molecularization is not reducible to how bodies are understood and intervened in, as Rose would have it, but that its epistemological purchase is much wider. It dominates how we conceive of life, of our surroundings, and our connections in the world. Molecularization is not limited but all-encompassing today. Molecules, in medicine, science, and society have become the ultimate "repository of truth" (cf. Zola 470); they are imagined as absolute, neutral, and objective.

With molecularization, also our concept of the body has received an 'update.' Molecules and molecular pathways today are the wheels and cogs in the machine of the human body, reductionism has not changed that. The bio-informatic metaphors used for example in the HGP are premised on older views of the body as mechanistic as well. We still see the body as a mélange of composite parts that can ideally be repaired or replaced, be used to manage our bodies inside (and out). What has changed, however, is that now this view of the body-in-molecular-pieces is no longer enough to capture the complexity of biological materiality: Instead, the mechanistic view of the body had to be incorporated into a more holistic, systematic view. The body now is both a machine and a flexible, interdependent system. As elaborated on in Chapter 2, the molecular body has been translated into an informational body during the past decades. Thacker's argument on biomediated bodies come to mind here. His broad, preliminary definition and understanding of biomedia "as the informatic recontextualization of biological components and processes," shows how the biomediated body, generally speaking, is informational, accounted for and known through data ("What is Biomedia?" 58, 77). Patricia Clough's reading of Thacker shows how biomediated bodies and molecularization are related: the biomediated body is a "recent complexification in bodily matter at the molecular level" that more apparently and productively uses the potential of the informational body (214-15). The biomediated body in Thacker's and Clough's renditions is informational only because it is molecular. Molecularization is a necessary precondition for bodies to 'become' data, it makes their biological materiality quantifiable. A prime example for this connection is genetic testing. Only the knowledge of the molecular structure of genes, the sequences of nucleotides, makes

In this world of molecular exchange, anxiety about our bodily integrity is also heightened through the (imagined and very much real) molecularization of pathogens: contaminants are invisible and highly mobile, so that suspicion abounds. A perfect example of this anxiety about molecular exchange is the outbreak of the Covid-19 pandemic in 2020. 
it possible to turn the material into abstract data and to calculate deviations and changes in those sequences through numbers and probabilities. This 'biomediated' body - as both biological and informational but inherently molecular - arguably is the most common form we encounter the body in contemporary contexts of self-directed tracking, testing and enhancement.

\section{Transparency and Molecular Plasticity}

Whether in the guise of epistemological shift, enhancement, or information, the narrative of molecularization has two prominent consequences. On the one hand, it creates a new form of transparency. The "transparent body" (Chrysanthou 469) can be seen as a direct outcome of the larger process of "getting under the skin." The development of ever more sophisticated medical imaging and information technologies have made the body transparent in the most literal sense. This transparency now extends even to those spaces and structures in the sub-microscopic regions and is facilitated to a large extent by information technologies that allow for elaborate simulations and animations. Digital programs that calculate the structures and folding of proteins are just one example commonly used in biotech research today. ${ }^{6}$ Here, again, biology, and bioresearch for that matter, is focused on both the materiality and the informational substrate of molecular processes. Molecular knowledge, thus, creates new, to use Thomas Lemke's words, "spaces of visibility" ("Disposition" 555) in which information about the body and its inner workings is made 'transparent,' intelligible, imaginable. ${ }^{7}$ In consequence, what was previously unexplored territory has now been carthographed, categorized and communicated. The molecular processes of the body have become topics of public discussion and deliberation - a significant part of everyday life, reflected also in their recurrent appearance in popular media and pop-culture. However, this transparency, as of now, requires experts and expensive equipment to interpret and explain it: Without this intermediate step of translation, the images and narratives circulating in (popular) culture would not be accessible for the lay public. This is where DIY biology and medicine, as will be developed in the later chapters, offers its most potential.

The other prominent effect of the molecularization of life is what I want to summarize as the molecular plasticity of the human body. Molecular plasticity denotes the idea that the human body today is conceived of and represented as malleable on a molecular scale. This concept draws heavily on Nikolas Roses assertion of the productive potential of molecularization for interventions into human bodies to realize their full potential. Rose writes: "Life now appears to be open to shaping and reshaping at the molecular level: by precisely calculated interventions that prevent something happening, alter the way something happens, make something new happen in the cellular processes

6 There is a large array of software used to predict the structure of a protein from its amino acid sequence - a good example of the collaboration between biology and computer sciences. These programs are useful as educational tools but also to design new enzymes and drugs in medicine and biotechnology.

7 These spaces of visibility, as Lemke argues, do not just concern individuals: Rather, genetic diagnostics offer predictive information about individuals but also their descendants, creating a "new, transgenerational transparency of the body" ("Disposition" 555). 
themselves" ("Politics" 16). It is the minute details of life that today are conceived of as malleable entities. Regenerative medicine is one of the examples in which molecular plasticity is most tangible in contemporary biomedical research. Medical interventions that aim to restore and repair damaged or 'malfunctioning' cells and tissues - such as gene therapy, immunomodulation therapy, stem cell research or tissue engineering require us to view the body as plastic, with exchangeable parts and amendable processes. As such, they seem to offer some of the greatest promises for curing previously incurable diseases, such as cancer or Alzheimer's disease. At the same time, interventions on the molecular level carry with them, in a posthumanist view, the possibility of changing forever what counts as human: It potentially allows humans to shape human evolution (Shaw 289). As such, they are bound to also have deep-reaching cultural repercussions.

One of the more negative repercussions might be a process of objectification that is bound to set in when molecularism dominates our conception of life and the body, turning people into mere molecular objects. Henrietta Lacks could be read as an extreme example of this type of objectification: One of the most notorious immortal cell lines in laboratories, the HeLa line, had been used for decades without 'credit' to its origins. In 1951 Henrietta Lacks, an African American woman, had to undergo treatment for cervical cancer during which her cancer cells were collected and later cultured without her consent. Lacks died in 1951, but neither she nor her family were notified about the cell line's existence until 1975 or compensated for the continued use of her cells also in commercial endeavors. ${ }^{8}$ Lacks, we could claim, was objectified and depersonalized through her cells, her molecular structure was traded as a commodity. The HeLa line can also be read as a symbol of the pervasive racism and neglect of patient rights that was prevalent at the time of its creation. Moreover, the focus on molecules might force individuals into the position of an observer of their own body, a manager of its molecular needs and predispositions in a process of self-objectification: But habitually monitoring the bodies' molecular status-quo, like the habitual monitoring of their bodies that women still experience in contemporary culture, can lead to feelings of insufficiency, shame, and anxiety.

\subsection{The Molecule as a Sign and a Promise}

While the previous subchapter recounted how biology and life itself came to be viewed primarily in molecular terms and consequently were imagined as a plastic entity, this chapter now wants to briefly consider how molecular biology makes meaning in the public manifold: The focus here is twofold, looking at molecules, especially genes, as both signs and promises. The cultural semiotics and communicative potential of biology, I would argue, is the clue to how we make meaning of biology in public discussions. Thus, I will here look at some of the imagery, metaphors, narratives that highlight the

8 Her life, the proliferation of the HeLa cell line and the story of her descendants are recounted by Rebecca Skloot in The Immortal Life of Henrietta Lacks (2010). 
cultural primacy of molecules. ${ }^{9}$ Especially the discourses around genes as texts, codes, books during the $20^{\text {th }}$ century have shaped how we conceive of molecular entities as signs or symbols for something more. They refer to (molecular) vulnerabilities that frame the body as endangered and as in need of pre-emptive and preventive measures. This gives rise to a lot of affective structures that shape our approach to our bodies and biological materiality: Affects such as promise and anxiety are primary forms of making use of biological knowledge; the meaning that is made is affective meaning, carried in individuals and collective atmospheres.

But first let me return to the question of molecules as signs or symbols, of the relevance of semiotics for the cultural study of biology. Symbolic activity and sign processes - like analogies, metaphors, symbolism - have naturally been part of Cultural Studies approaches due to their goal of identifying and interpreting how meaning is made through communication, broadly understood. The semiotic turn in Cultural Studies allowed for many phenomena to be regarded as 'texts' and thus subjected to a more or less semiotic analysis. Biology and molecules, understood as broadly textual, are no exception here: With the dissemination of biological insights into the cultural sphere this knowledge has become an intrinsic part of cultural meaning making about the fundamentals of (human) life. ${ }^{10}$ But also in science the importance of semiotics has been realized since the 1990s with the rise of biosemiotics as a discipline in its own right. Biosemiotics, broadly, refers to the study of signs, communication and information in living organisms (Cammack and Attwood, Oxford Dictionary of Biochemistry and Molecular Biology, 72). This new approach combines semiotics and biology, prompting both disciplines to expand their own self-understanding: biology, as the Biosemiotics journal maintains, needs to recognize and incorporate the semiotic nature of life and semiotics needs to expand its definition to include biological signs. Biosemiotics, thus, looks at how symbols and signs are used by living organisms to communicate information on molecular and cellular levels. Here, however, I want to focus on the cultural side of things: It is biological knowledge that shapes how we make cultural sense of our embodiment.

Molecules have become what Illana F. Silber calls "master metaphors," as they play a central role in shaping and controlling both biological research and cultural understandings. ${ }^{11}$ Deborah Lynn Steinberg, in her analysis of Genes and the Bioimaginary (2015),

As Henk ten Have has pointed out, it is important to make a clear distinction between public perception and scientific reality or debates: The public perception is not always congruent with scientific facts and state-of-the-art knowledge (295). Rather, I would argue, that often the public perception - despite attempts to communicate new insights - lacks behind scientific understandings.

10 Wittgenstein can be useful here to point out how the words and concepts we use changes how we perceive things, also in research settings (cf. Chapter 2.1).

11 To quote Silber: "Both humanities and the social sciences, in this respect, have been deeply affected by the emergence and diffusion of new 'master metaphors', as I have termed it elsewhere, i.e., metaphors not simply used to adorn or enliven sociological writing, but actually playing a central role in the shaping and controlling of sociological theory and research (Silber, 1995b). I have in mind, for example, the impact of such potent literary metaphors as 'culture as text' and related ideas (i.e., genres, scenarios, narratives), as well as a whole range of economic (e.g., 'capital', 'market', 'goods'), spatial (e.g., social 'space', 'fields'), and artistic (e.g., 'repertoires') metaphors, 
looks at genes as a "site of signification" (italics in original). "Signification," for her, is part of a social semiotics in that it is understood as both "embedded in and constitutive" of the social field (Steinberg 4). I want to generalize her idea by looking at all molecular processes as such "sites of signification" in that they signify through signs or symbolic means certain meanings and associations. One consequence of the molecular gaze is that today we have started to actively seek out, look for those signs as clues in our quest to understand and optimize our bodies. Cultural semiotics can help to analyze these processes of signification, it is a tool that allows us to render hidden messages visible and therefore acts as an "agent of revelation" (Chrysanthou 475).

Genes have in the decades since the decoding of their structure become an important site for the intersection between culture and biology. Almost immediately they gained a high public presence, a growing fascination with genetics that was reflected in the wealth of media coverage (Have 295). The Human Genome Project (HGP), and especially the revelation of its results, were highly mediatized, and in the subsequent years findings about genes, disease etiology and mundane traits have made headlines as well. ${ }^{12}$ But not just in the media, also the popular science sectors of bookshops have been populated by accounts of genetics: A recent example being Dawn Field's Biocode: The New Age of Genomics (2016) or more prominently Siddhartha Mukherjee's The Gene: An Intimate History (2016). ${ }^{13}$ This public pervasiveness makes the gene probably one of the most prominent molecules, a well-researched example of the semiotic processes at work in molecular biology and therefore a good illustration for my analysis here. Following Steinberg, I see the gene as being configured as both a tool for biotechnological interventions - "a site of practice" - and a symbol (Steinberg 1). This symbolic or signifying quality of the gene is closely connected to the discourses used to describe it.

\section{Let's Talk About Genes}

For that, a short excursion into how we have talked and made sense of genetics for the past few decades is in order. During the early phase of genetics in the 1950 s and

combining or competing with older metaphors such as 'organism', 'system' or 'code'." (Silber 222) I, however, make use only of her general idea.

Often, these accounts also perpetuate a certain genetic determinism in the 'gene for discovery'-narratives used, examples include schizophrenia, depression, height, athleticism, and other diseases and traits. In many cases, the correlations that lead to such reporting, however, remain murky and unclear. cf. also Duden and Samerski, Nelkin and Lindee, Shea, Tambor et al.

13 In 2016 Siddhartha Mukherjee, the author of the Pulitzer Prize-winning The Emperor of All Maladies, published The Cene: An Intimate History. As with his earlier account of cancer, in this prime example of non-fiction science writing Mukherjee tries to construct not just a scientific but also a cultural history of genomics. He thus tries to bridge the divide between scientific narrative and its cultural and social implications. Mukherjee emphasizes both the personal ramifications of genetics and genetic knowledge and their intersections with cultural and political questions, such as race, identity, sexuality and choice. He stresses that the gene is as much a scientific concept as it is a cultural and political tool (Mukherjee 128). As a cultural history, his book puts a lot of emphasis on the contexts of the discoveries and combines accounts of persons, experiments, institutions and technological progress with social considerations and cultural representations. As such, Mukherjee both deals with a molecule as a cultural phenomenon and the process of popularization itself. 
reaching all the way into the 1990 s and early 2000 s, the dominant lens through which genetics was conceptualized was that of a text. This stance was to a large degree influenced by an overall preoccupation with narrative and linguistics in academia and public culture. Tropes and metaphors such as letters (to describe nucleotide bases), words (as stand-ins for genes), chapters (for chromosomes) and lastly the 'book of life' (the whole genome) where commonly used to explain DNA. Such metaphors as books, recipes or blueprints give DNA a linguistic quality, imply that there is something like a grammar, a rule book (Thacker 65 ). This view was dominating the public understanding of genetics for almost half a decade. All that supposedly changed with the publication of the results of the HGP in 2001 and 2003, which had thrown much of what was presumed in genetic research into disarray. Scientists had found fewer genes than expected, and fewer even than would be needed to account for human differences. Instead of the previously expected 'one disease/trait-one gene' association the scientists had hoped to crack, they had to acknowledge that the correlations between genotype and phenotype were much more complicated. This acknowledgement led to a fundamental change in genetic thinking and terminology, from genetic determinism (straightforward causeresult relationships) to genomics and epigenetics, that focused on interactions along the metabolic pathways, gene regulation and environmental factors. Attention now turned to Single Nucleotide Polymorphisms (SNPs), interactions between DNA and (cell) environment, non-coding DNA regions, alternative splicing and other complexities. ${ }^{14}$

It is small wonder then that with the completion of the HGP, we also see a change in how DNA was communicated: the dominant metaphors changed from those associating the genome with ultimate answers - the Rosetta Stone, the Holy Grail, a master text to those carrying more uncertainty, such as a parts list. This idea gained momentum in the early 2000s: The reconfiguration of DNA as a parts list - still a text-based metaphor, as we may note - removes a sense of order and presumption of specific instructions in favor of a simple collection of elements: a digital list, a databank of facts (Roof 112). This shift in terminology goes hand in hand with a shift in logics towards systemic views, Roof argues. The "parts list" was a hybrid metaphor that connected a symboland story-based logics with a worldview dominated by systems and networks (Roof 11112). The conceptualization of DNA as a code shifted from linguistics-based one to an informatics-based account. In this new informatic view of DNA as code, as Thacker argues, DNA "has no grammar, it does not signify, and there are no words," it is pure combinatorics. But this view though prevalent today is no less problematic, as it implies that DNA "only calculates, operates and parses" (Thacker 65). It is no less deterministic than the textual view of DNA. Here we cannot only see that the metaphor of DNA as a

14 Broadly, Single Nucleotide Polymorphisms (SNPs) are changes in single nucleotides at specific positions in the genome. Alternative splicing describes a process during gene expression through which a single gene can code for multiple proteins: in some cases exons (regions) of a gene might not be processed into mRNA, meaning that after translation the proteins will have a different amino acid sequence and (potentially) biological function - explaining the higher diversity in proteins in relation to protein-coding genes. 
(language) code has taken on a new connotation, but that all these metaphors tend to take on meaning of their own (cf. Stevens 41 ). ${ }^{15}$

It is through and in representation, following Nelkin and Lindee's argument, that the gene has become a "cultural icon, a symbol, almost a magical force" (2). As a symbol and metaphor, the gene is more than its biological structure and has gained cultural meaning apart from its biological definition: The pervasive genetic imagery has turned the gene and its visualization into an entity that is inextricably tied to questions of identity, community and relationships (Nelkin and Lindee 2,16). ${ }^{16}$ The gene becomes more than what it is, gains an extra layer of meaning, of social awe and wonder. DNA as sign and symbol stands in for larger ideas. For example, even though scientific research departed from a structuralist model, representations of DNA in the public often still use a structuralist, linguistic model as a simplification to convey complex process in familiar terms; a repetition that, seen critically, urges the public to keep thinking in an outdated but comforting language (Roof 65 ). These linguistic approaches suggest that "DNA is readable, translatable, writable, editable, and copyrightable," that "genes can be manipulated, rewritten, and ultimately owned" (Roof 90, 16). This form of understanding, I would argue, contributes a great deal to the idea that we can use our genome to shape our phenotype, to the assumption that that if we know our genome, we can somehow control and manage it. ${ }^{17}$ Already the word gene, according to Barbara Duden and Silja Samerski, has transformative power, it reinterprets the subject in corporeal, fleshy terms, thus has the capacity to "perform a shortcut between genotype and phenotype." This reflexivity merges the somatic and intimate with statistical probabilities and risks, thereby blending "the concrete and abstract, visible and invisible, tangible and conjectured" (168). All this, Duden and Samerski argue, gives the gene a symbolic function that fuses bodily substance with personhood and risk calculations (167).

\section{Signifying Vulnerability}

Dumit and Burri assert that the gene produced a "layered series of deferrals and guesses," instead of catering to its conceptual fantasy of control (223). Instead of providing order and definite knowledge, it opened up a void of uncertainty and vulnerability. This, furthermore, is true not just for the gene - this most famous

Another metaphor often used in genetics, that of DNA as the "secret of life," reconfigures DNA as a sacred text, the locus of identity and personhood, a "soul-like entity" - given its analogy to theological concepts - and the location of the "true self" (Nelkin and Lindee 39-46). Here, the metaphorical expansion of a molecular structure gives it a mystical quality, shifting the meaning we make of it. Nelkin and Lindee wrote this argument as a criticism of the pervasive genetic essentialism in science and society. Cenetic essentialism, they argue, reduces the self to a "molecular entity," equating complex beings with their genes (2). Their account is useful to look at the consequences of popular representations for the understanding of science and biology and certainly also for delineating the many different shades genetic essentialism took in public discourse in the 1990 .

17 Deborah Steinberg argues that despite the promises of language metaphors to confer the ability to read genes on lay persons, of democratizing science, in reality genetics rarefies, fosters "public dependency on a much reduced pool of professional readers" - those competent in the language of genetics - and thus heightens "the inequalities that constitute scientific expertise and scientific professions" (Steinberg 55-59). I will return to the question of lay expertise in later chapters. 
molecule - but also other molecular structures and pathways are still dominated by a high level of uncertainty about clear-cut cause-effect relations or at least so fraught with complexities and multiple possible sites of disturbances that control and order remain a fantasy. But, again, as so much research has already been done on DNA and genomics, it is most useful as a quick example.

The developments after the publication of the HGP, especially the new focus on SNPs, epigenetics and gene regulation, "encourage[d] us to understand ourselves as a set of risk factors," that became part of our identities and our aspirations (Stevens 307o8). This identification as "genetically at risk" can either take the form of a self-identification or be ascribed by others, often medical professionals or experts. ${ }^{18}$ DNA here signifies susceptibility and risk, which ironically are not biological matters of fact but only correlations and informed guesses, derived from calculating probabilities based on associations between genomic changes and disease expression. Still, the "premonitory knowledges" derived from genetic testing create an obligation to act in the present in relation to potential futures (Rose, "Genomic Susceptibility" 147). The overall message is to act responsibly, towards the self, the family and wider community. The prevalent discourse of risk and susceptibilities, thus, create new (perceived) molecular vulnerabilities that frame the body as endangered and as in need of pre-emptive and preventive measures. This new vulnerability here takes two forms: It makes visible our inherent vulnerability as humans, but also creates a new situation in which some individuals those with 'defects' in their genetic code - will be more vulnerable than others, physically and emotionally, more prone to dependence, illness, death, rejection, oppression, or exploitation, exacerbating a perceived loss of control and agency (cf. Mackenzie et al. 9). For example, being diagnosed with a genetic predisposition for Alzheimer's disease not only increases our perceived vulnerability to forces within our body (succumbing to discourses of genetic essentialism), but it also exacerbates a feeling of powerlessness in the face of our own biology, confronts us with a new (possible) future defined by dependence and loss. It makes us feel our inherent vulnerability more desperately, more prominently.

Taking this one step further we can see that the molecules as signs also generate affective reactions, primarily promises and fears. As Hallam Stevens argues promises are an inherent part of biotechnology, making them also a part of DNA and genetic technologies: They are often oriented towards the future and generate expectations (both on a social and an individual level) about biotechnologies and their applications. But they also influence how people understand science, "what [they] expect, what [they] desire, and what [they] fear" (Stevens 8-9). Genetic diagnostics, for example, promise certainty (while creating uncertainty) and accuracy, but also control over the previously uncontrollable (nature, chance, fate) (Lemke, "Disposition" 557). They promise to ameliorate uncertainty and prevent suffering. But, as we can see with molecular vulnerabilities, DNA is also a symbol that encodes anxieties about the future, the state of our bodies, our plans and aspirations: anxiety about the contingencies of human life and the vulnerabilities that come with it. Here, we can see that also affective structures and

18 Once again, one can be susceptible also on other levels than the genetic one. This argument thus is very much generalizable. 
affective responses shape how we make meaning of biological knowledge: The affects that arise such as promise and hope or fear and anxiety show that much of the meaning that is made is affective meaning, at least on a second order. What is more, these "affective atmospheres" (Ben Anderson, Encountering Affect 21) are very much a collective condition: As affects do, they cross between bodies and things, arise out of individual and mediated encounters, and forge communities where individualistic thinking had previously questioned them.

\section{The Story of the Happiness Gene}

Let me put all this in a bit more practical context by looking at an example: Richard Powers' 2009 novel Generosity. An Enhancement. On the first look, Generosity is a 'gene for' discovery story - reminiscent of more deterministic views of genomics. This realist novel is set in a parallel Chicago and centers around Thassadit ("Thassa") Amzwar, a refugee from the Algerian civil war now living in the US, who seems to always be "exuberant," blissful, content and happy, radiating a "chronic, viral euphoria" (36), nothing seems to faze her. Thassa is studying at a local college where she meets Russell Stone, a (former) writer and Thassa's writing teacher. Stone soon recognizes that Thassa is always happy and, due to his own unhappiness and discontentment, seems to become almost obsessed with Thassa's happiness and its causes.

When Thassa is almost raped by a classmate, she talks him out of it and the classmate voluntarily turns himself in. During the subsequent investigation, Stone makes the mistake of mentioning her unusual psychological disposition to the police and the extraordinary story is taken up by the local news outlets. Due to these reports Thassa draws the attention of Thomas Kurton, a geneticist following his own fantasies of human enhancement, who is studying the molecular and genetic basis of happiness and psychological well-being. After meeting her, Kurton gains Thassa's permission to study her genetic make-up and include her in his recent study, for which she turns out to be the last missing proof of his theories, the perfect research subject. Both men's curiosity about Thassa - Stone's interest in her past, heritage and personality, and Kurton's interest in her biology - means that, at least in the beginning, we encounter Thassa primarily through a male gaze (Stone's) and later a scientific gaze (Kurton's), both of which seem to objectify parts of her. When Kurton's research is published, using a pseudonym, it does not take long before people start to connect the dots from "Jen" to Thassa, so that Thassa now finds herself at the center of a nation-wide public craze, followed and hunted by the public and media alike. She encounters both disapproval and admiration, people camp out in front of her apartment, call her, want to know her secret. She becomes a "publicly traded commodity." ${ }^{19}$ When it all becomes too much, Thassa decides to flee Chicago, asking Stone to take her back to Canada, where she had previously lived with relatives. Soon, another news story breaks, this time about Stone having kidnapped Thassa, at which point she becomes almost suicidal and takes Stone's sleep and pain medication 
while he is out of the room. Thassa, the perpetually happy girl, thus ends up utterly miserable, broken by the public knowledge of her perceived 'biological' advantage.

As is typical for Powers' novels, Generosity is also a worthwhile treatise on genetics and the question of human enhancement. Genetics in this narrative is closely associated with, primarily Kurton's, fantasies of enhancement. Thomas Kurton seems to believe that happiness is the result of complex neurochemical pathways which are the result of genes. ${ }^{20}$ It is no wonder then that Thassa's happiness seems to be anchored in her genetic make-up. Something concrete, some sequences of DNA, come to stand in for something very abstract and subjective. Thassa's DNA, and thus her biological materiality, begins to stand in for more: for life happiness, psychological well-being, and potentiality. The gene as a molecule here signifies a promise of happiness, of malleability and the potential for change. ${ }^{21}$ In Kurton's fantasies we see visions of a better human life and a more adapted psychological disposition, of enhancing humans to perpetually feel content, happy, even-tempered. But, as the media coverage and the public craze about Thassa show, this genetic imaginary is not limited to Kurton. Rather, the public reaction makes this a collective reaction, the promises, hopes and sometimes also fears are infective. ${ }^{22}$ What is created, in the reports on Thassa and the imagination of the public, is a promissory atmosphere, in which one individual - or rather her biological disposition - is turned into a collective sphere of hope to escape into a utopia of perpetual contentment. ${ }^{23}$ It is therefore also on the level of affect, of affective atmospheres, that the public makes meaning out of the newly-found biological knowledge; it is this affective meaning that guides how Thassa's life and body are evaluated.

In the end, not just Thassa's 'fall' from happiness, but also the critical subtext of the novel prompt the reader to question the discourse of enhancement. Power's lets the moral debate about enhancement play out in the narrative by proxy through a conversation between a successful novelist and the geneticist Kurton, "a dialogue between two

20 In general, the discourse of genetics in this narrative is closely following current state-of-the-art in real-life science. The scientists in the narrative use an up-to-date conception of genes and heritability, visible in the many discussions and mentions of genetic complexity, epigenetic insights, gene regulation processes and the use of association studies. Much of this information about genetics is given in one of the many narrative strands that focuses on "The Genie and the Genome," a science TV show covering genetic enhancements and the happiness gene. Many of the other news media outlets in the story, after hearing about Kurton's study, however, report of the findings in a much more limited way: as the discovery of a 'gene for happiness' or the happiness gene. Press reports seem to employ the well-known formula of the 'gene for'-narrative to make better headlines and create more attention. The general understanding of genes in the fictive public, thus, seems to be rather outdated (Powers, Generosity 173, 181-184, 255-256).

21 Thassa claims people should stop looking for spirits in molecules (221): She critiques how excessive meaning is made of her molecular make-up, especially since it requires a jump from something very scientific to something "spiritual" and subjective.

22 Thassa sees happiness itself not as genetic but as contagious (Powers, Generosity 204). "Crimes of passion" (195) are committed in aftermath of the publication of the study, including physical violence and death threats.

23 For Thassa "Jen" is an "imaginary" woman (198). She disengages her 'self' from the publicly imagined utopian body. 
cultures" (137-41) at which's end stands the conclusion that there is no end point to enhancement because enhancement itself is always, and crucially, a future-oriented and promissory process of never-quite-there. Still, this narrative example shows how the gene as a sign and symbol takes on a whole layer of different meanings that shape the public's reaction and evaluation of it. No figuration of DNA, as Roof writes, is free from additional, even if subtle, cultural ideas, deliberately chosen or unconsciously inserted (15). Thassa, in this narrative, is an embodied utopia, a 'real-life' (in this fictive world), concrete example of a utopian vision: It is her material body that, due to a biological quirk, serves as a screen unto which age-old desires for happiness, contentment, positivity are projected - a projection that ironically makes her more susceptible, more vulnerable, especially to the actions and judgements of others.

\subsection{A Phantasy of Progress: The Perfect Body}

As Priscilla Wald and Jay Clayton write, human beings explore the questions that most concern them through fiction, through the works of imagination (Wald and Clayton $\mathrm{x}$ ). Imaging life on a molecular scale, here, moves closer to its phantasmic meaning, how we can envision applications of molecular biology and their effects on the human body. This chapter, in a first step, looks at how molecular biology and the human body are talked about in science fiction. My focus is on narratives that include bodily enhancements. These narratives provide good examples of how biotechnological progress is often intrinsically combined with some form of human enhancement, either through interventions or body-machine interfaces. Such narratives can tell us a lot about our attitudes towards biotechnologies and how they change our view of human bodies. However, as the title already tells: Science fiction means that these narratives are fantasies. Fantasies, according to Steinberg "imaginative projections, articulated in narrative form," when "leveraged" through affective relations speak to more "inchoate yearnings and anxieties" - phantasy (Steinberg 6). Such "phantasmatic projections," again, illuminate the inherent combination of narrative meaning-making and larger cultural anxieties and promises. As a second step, I illustrate how those phantasies of perfected bodies create the impetus for a new rendition of the on-going conceptualization of bodies and selves as projects, inspired by techno-science discourse around new and imagined technologies. This view of the body as project, subject to technological intervention, not only has social and cultural repercussions but very much material ones. This chapter, thus, takes a first step to bridge promise and plasticity, or culture and materiality.

To gauge the implications and meaning of such phantasies of perfected bodies, I want to refer to Bernadette Wegenstein's argument about The Cosmetic Gaze employed in our contemporary makeover culture. Wegenstein originally looks at cosmetic techniques of enhancement, but her argument, I would claim, can very much be taken as a basis for much of the (self-)modification techniques in widespread use today, those that leave the superficial space of the cosmetic and enter the deeper level of the molecular. ${ }^{24}$

24 Even though Wegenstein is focused on the theme of "beauty," the cosmetic gaze can be argued to underlie the whole idea of perfectibility. It created, in the context of first superficial and then 
Wegenstein argues that today we look at others and ourselves through a cosmetic gaze that scans all bodies as in need of improvement and modification to become complete. In contemporary culture, the makeover discourse in beauty propagates the possibility to attain a "better beautiful version of the self" through improvements so that the imperative to alter the body (also through violent means) is readily embraced (Cosmetic Gaze 111, $151,184) .{ }^{25}$ This cosmetic gaze, Wegenstein claims, is circular in that "being surrounded by made-over bodies produces a desire for one's own makeover" (The Cosmetic Gaze 2). We are surrounded by such made-over bodies also through the media (119). Images and narratives of enhanced bodies, in whatever form, affect the viewer's body image, vulnerability and readiness to change (The Cosmetic Gaze 119). A prominent example, also subject of Wegenstein's analysis, are make-over shows and reality TV-formats like The Swan or The Biggest Loser, both of which have been adapted also outside of the US. ${ }^{26}$ These shows confront the viewer with successful make-over narratives - and in the end made-over bodies - that can increase their desire for their own transformation. Here we come to see why the cosmetic gaze is important in this chapter: Mediatized, fictive or not, depictions of human bodies can act as agents of normalization as well as catalysts for desires and fears, so that following this circular structure, we begin to see human bodies as open to change, as open to perfection. Representations allow people to imagine themselves otherwise. This form of normalization paves the way for transformation, modification and enhancement. Such fantasies of perfection can also be found in Powers' Generosity. Making humans emotionally more fit and stable, constantly content is the major goal in Kurton's fantasies of human enhancement. In Powers' realist novel, however, these enhancement fantasies are not carried out but rather disputed heavily in society, even though they do spark a collective, promissory atmosphere. Science fiction novels, on the other hand, have more leeway when fleshing out those fantasies.

surgical interventions, the underlying view of the body as always open for improvement. The enhancements and interventions possible, however, have moved into the biochemical realm. Still, the cosmetic gaze and its view of body and self can be said to form the basis for those possibilities. Surface and surgical interventions into the materiality of the body are the basis for biochemical ones. The connection between outside and inside change and transformation that Wegenstein stresses for the cosmetic gaze is also involved in biochemical enhancement, especially in the notion of carving out the "true and authentic" self. The cosmetic gaze, thus, can be considered as a precursor of the molecular gaze-both are directed at the self and others, but the cosmetic remains on the surface of the body, while the molecular gaze reaches deep into its biological make-up. Both, however, are still in use today, with the cosmetic often being reframed through the molecular.

25 This readiness to improve the self, indeed, is not a very new phenomenon but one that has accompanied human cultures for centuries - make-up was already used by the Egyptians and corsets are a good example of painful means used to attain a propagated ideal of beauty. But, in the $20^{\text {th }}$ century cosmetic surgery has become one of the most widespread and important applications of the cosmetic gaze, self-improvement and popular consumer choice (Wegenstein Cosmetic Gaze).

26 Both have been met with vehement criticism. The Swan, featuring extreme make-overs including cosmetic surgery ran for two seasons in 2004. The Biggest Loser (Silverman), though also criticized for its approach to weight loss, ran more successfully for 17 seasons, from 2004 to 2016, with a reboot said to air in 2020 . 


\section{SuperHero / SuperHuman}

A widely distributed genre, science fiction can only be understood as a "family resemblance concept" with blurry edges and many subdivisions, ranging from films, to TV, novels, comic books and graphic novels, to popular music, art and architecture (Erickson 193-96). Broadly understood, science fiction is a play of ideas - a construction of what-ifs - and an exploration of new concepts, both in science and society. ${ }^{27}$ The (future) societies created in many science fiction works engage with diverse social issues, play out alternative social orders and reframe them so as to open up new perspectives. But science fiction also works through the imagination and imaginary. It demonstrates which uses we can imagine for science and technologies, what transformations those uses might affect, and how they will change what it means to be human (cf. Stevens 370). ${ }^{28}$ As such science fiction is not just about technologies or about fictive engagements with the future, not about science or fiction but rather about human life. Early narratives such as Mary Wollstonecraft Shelley's Frankenstein, Robert Louis Stevenson's Dr. Jekyll and Mr. Hyde or Nathaniel Hawthorne's "The Birthmark," show that they are not just future-directed and science-centered narratives - what we often presume them to be. Instead, they reach to the core of what is means to be human, to have a body. The figure of the human and its material manifestation take center stage in most narratives: where human bodies stand in society, how they interact and how they are shaped. As such, they are an avenue for thinking through different bodily states of being or body modifications, for imaginative engagements with fundamental questions such as the different value assigned to differently abled bodies, the demarcations of 'humanity,' or the effects of social configurations on human bodies. Christina Bieber Lake writes that fiction writers become "prophets" by imparting their visions of the future in writing: "In fiction, they flesh out the worlds we think we want; they imagine the outcome of our deepest dreams; they challenge the desires that fuel our decisions." (Lake xviii) These fictive engagements are thus important avenues for debating important (cultural) questions.

Superhero or 'superhuman' narratives are a form of science fiction that is not just intensely popular today but also inherently concerned with humans that are 'more than human,' enhanced bodies in one way or the other. These imaginative engagements with human physical extension have been around since the birth of comic books and especially their Golden Age in the 1930s and 40s. Many of the long-running superhero comics and subsequent franchises have become a staple of popular culture, with a distinctly American flavor to them. Superhero narratives, thus, could also be considered as

27 According to Mark Erickson, science fiction is very much a product of modernity: Speculative tales about technology, people and societies of the future emerge only in the $19^{\text {th }}$ century. Imaginative engagements with these topics, he posits, needed the overarching influence of technology on all spheres of society (Erickson 190).

28 The imaginative engagement with science and technology aligns with broader ideas of science as a narrative practice, a "story-telling enterprise" (Steinberg 28). I would argue that science becomes a narrative practice especially in cases where narrative is what mostly sustains and inspires science: Where the imagination fuels scientific inquiry or when what is imaginable transcends what is doable, as in genetic enhancement. 
essentially American, visible also in prominent heroes such as Captain America, a product of the Second World War.

The X-Men is a comic series, invented by Stan Lee and Jack Kirby at Marvel Comic (since 1963) that was turned into a successful movie franchise (20 ${ }^{\text {th }}$ Century Fox), with a total of 13 released or upcoming films. The success of this franchise is often credited for paving the way for other superhero franchises based amongst others on Marvel Comic's characters, such as Spiderman, the Fantastic Four, Superman, or The Avengers. In the X-Men universe natural mutations occurred, altering some people's DNA and giving them new (super-human) abilities such as fast healing, telepathy and telekinesis, enhanced strength, the ability to generate and control magnetic fields, minor or more radical changes in physical appearance (fur, wings, tails, skin color and so forth) (S. Lee and Kirby). The series thus includes many differently abled bodies, most of which can be considered enhanced in contrast to the wider 'human' community. The changes, it is claimed, are the next step in human evolution. All mutants have a genetic mutation, the X-gene, that sooner or later will reveal the individual's abilities. The mutants come from all strata of society with some living openly, others more covertly. The potential for conflict or rivalry between enhanced (though naturally) and human is one of the common staples of the series. Peaceful coexistence and equal rights are discussed alongside social unrest and violence against minorities, with different scenarios often playing out in different time lines or alternative universes. The X-Men films thus reflect many of the current cultural and social issues in one way or the other: racism, prejudice, and discrimination are discussed as well as diversity, sexual identity and gender conformity, subcultures and religion.

But more than that, they are a fictive engagement with natural plasticity: The bodies of the mutants show a diversity of mutations and modifications that make them 'more than human' but also take the idea of plastic bodies to a hyperbolical conclusion - bodies changing on their own accord. Other characters in the Marvel Cinematic Universe have different genealogies, ranging from superheroes with 'superhuman' abilities mostly due to fusion with technology, human experiments, exposure to body-altering substances, supernatural occurrences and magic abilities, or alien ancestry. All of them, however, show bodies that are more able than the average human. Such superhuman/superhero narratives see human bodies and human abilities as a continuum of possibilities and bodies as malleable entities. As such, they are probably one of the most prominent cultural representations of enhanced human bodies that - in Wegenstein's circular gaze - might serve as blueprints for human desires for change, inspiring awe, wonder and in some cases also real-life interventions into the human body, as the example of 'grinding,' a sub-movement of the biohacker scene, will demonstrate in Chapter 9.

Amplifying cultural beliefs, the "prophets of the posthuman" will go as far as the imagination will carry them while at the same time bringing contemporary issues to possible, imagined conclusions. How we talk about molecular biology and biotechnologies resources fantasies of perfected bodies in which the "manipulation of the plastic matter of the organism" (Landecker) is normalized. Such fantasies are also phantasies of transcendence in which humans, through science, can transcend their limitations, no matter if that means overcoming disease, achieving desired abilities or correcting social wrongs (Steinberg 163). The authors and creators of science fiction narratives 
have constructed 'what-ifs' about the increasing ability to shape human life also on genetic scales, resulting in idealizations of perfected human bodies. The "cosmetic gaze" created and perpetuated by such imaginative narratives, however, is also a moralizing and normalizing gaze that promotes a particular ethic of improvement. The result of which is a continuous process of self-adjustment according to internalized norms and expectations of what a body is or should be able to do or look like: The attempt to conform to some imaginative standard of ableism, fitness, health and strength. This, of course, is not exclusive to science fiction. Rather, it is a common cultural ideal that in science fiction is much more closely associated with budding technologies in biology and medicine.

\section{Playing with Possibilities}

At this point, the imagination intersects with a conceptualization of the body that has been referenced again and again in academia: That of the body as a project. Writers from widely different theoretical directions such as Anthony Giddens, Chris Shilling or Nikolas Rose, to mention only a few, have re-framed the body and self as a project to be worked on. The body has become a site of self-expression. For example, in his version of the "body project" Shilling argues that the body has become "a project which should be worked out and accomplished as part of an individual's self-identity" (5). Giddens similarly argues that the body is integral to constructions of the self (Modernity and SelfIdentity). Such arguments see bodies as surfaces to be read by others: body modification here becomes a form of experimentation with the body, an expression of meaning and a form of self-determination in postmodern culture (Pitts 31).

Wegenstein takes up the idea of the body as a project in her consideration of the cosmetic gaze: With the body no longer a "given but a platform to invest in" (Cosmetic Gaze 131) - with the expectation of a return on this investment, I should add - it becomes framed through the cosmetic gaze as an "incomplete project" that can and should be intervened on and in through technologies of enhancement. The return on this investment is the perceived revelation of a "true" or authentic self and the realization of its natural potential (Cosmetic Gaze 109). Here, Wegenstein observes a short-circuit between outside modification and inside transformation (Cosmetic Gaze 2): Work on the body equals work on the self, changing the body is seen as transforming the self, so that the body comes to reflect the self. Or as Rose put it: The body, our material corporeality, is central to our understanding of what and who we are; our bodies have "become ourselves" (Politics of Life 105).

The "somatic individuality" (Rose) created through the process of individualization and medicalization during early modernity means that today humans are understood through their corporeal existence and biology. It is these that is acted upon. Biomedicine and technology have at the same time changed what we are promised, what kinds of people we can imagine to be, so that our molecular corporeality is the target of our dreams and desires as well as the techniques employed to "improve ourselves" (Rose, Politics of Life 25-26). These ideas have brought into flux what was previously considered as given: Biology is no longer fixed, no longer destiny but complexity, opportunity, probability (Rose, Politics of Life 51). Such a stance of course not only has 
social or cultural implications (for our notion of the self and identity), but very much material effects. The body is turned into a, quite literally, material 'project' that is not worked on in some abstract sense of self-transformation but on the level of its flesh and blood, its molecular make-up and in some cases molar integrity. This notion of the body as a malleable entity that can be used as a tool for self-expression creates new possibilities for self-making through bodies, potentially less normative and more creative ones. $^{29}$

It is no wonder that such a view of the body invites and inspires transhumanist, cyborgian fantasies of extension and transcendence. Already H.G. Wells - the science fiction author famous for The Island of Dr. Moreau (1896) - wrote at the end of the $19^{\text {th }}$ century:

A living being may also be regarded as raw material, as something plastic, something that may be shaped and altered, that this, possibly, may be added and that eliminated, and the organism as a whole developed far beyond its apparent possibilities ... a living thing might be taken in hand and so moulded and modified that at best it would retain scarcely anything of its inherent form and disposition; that the thread of life might be preserved unimpaired while shape and mental superstructure were so extensively recast as even to justify our regarding our result as a new variety of being. (Wells 36)

Following his interest in the molding and shaping of humans, he believes that they can be modified from their current state into something that could very well be perceived as a "new variety of being." Such fantasies might take the notion of the 'project body' to an extreme. They are, however, the logical conclusion of the belief in an individual's right to create their own body-projects and highly prevalent imaginaries of human extension in the cultural manifold. In fact, this idea will be taken up in a later case study on extreme body-modifications through technologies (Chapter 9).

In summary, discourses that position the body as a project to be continuously worked on contribute to a cultural politics of life focused on self-management, material intervention and individualized transformation. The body today is understood as a medium that we can work with to achieve individual goals, such as self-expression, rebellion, conformism, individualism. It is no longer a given but expected to be worked on and with during the human life span, subject of transformations and optimizations according to some cultural ideal. The body thereby has become central to questions of identity, community and difference, but also to value judgements and normalizations. The body is perceived as wax in our hands: we can mold it, shape it, cut it, dis- and re-assemble it according to our linking. We can transform its outer appearance and its inner recesses; we can use short-term modifications or long-term augmentations. The body has become a source of endless play, with forms, shapes, identities and embodiments. It is not just a repository of truth - harboring somewhere deep inside the 'true' self to be carved out as well as revealing an objective truth about the corporeal status quo - but also a repository of possibilities, pregnant with desires, anxieties, dreams and fears. This project, however, is destined to be unfinished. Just as fantasies

29 Pitts in In The Flesh (2003) diagnoses a similar creative potential for body modifications, also more extreme ones. 
of perfection never reach their goal, also the body as a project is indefinite. The body here becomes the ultimate utopia, existing only as some imagined end goal. ${ }^{30}$

\subsection{Coda: "Homo Deus" - A Promise of Tomorrow?}

"And having raised humanity above the beastly level of survival struggles, we will now aim to upgrade humans into gods, and turn Homo sapiens into Homo deus." (Harari, Homo Deus, 24)

Following his bestselling book Sapiens. A Brief History of Humankind (2014) Israeli historian Yuval Noah Harari published Homo Deus. A Brief History of Tomorrow in 2016. This book sets out to continue the evolution of Homo sapiens started in Sapiens into a hypothesized future. One of his main premises is that humankind will in the future intensify its attempts to reach perpetual happiness, immortality and God-like powers. This book - and that is why the title of this coda draws heavily on its title - is one of the most widely-read imaginations about the future of the human species in the past years. As such, it combines much of what is referenced in this chapter: the advancement of biotechnologies and concomitant possibilities to intervene in human bodies on smaller and smaller scales, the promissory narratives created around biotechnologies as well as humanity's innate yearning for and ability to change and shape itself, the fantasies that drive those desires. Homo deus, the god-like human, is the imagined, the promised outcome of the trends we see today, claims Harari. This change, he says, will not happen in "a day or a year" (56) but will be the result of incremental changes over a long period of time; changes that for him have already begun. Biotechnology, understood through culture, always carries with it the promise of a different tomorrow, of applications real and imagined that could cure or enhance and that could change our very definition of humanity. Whether this tomorrow is judged positively or negatively is beside the point of this book . Rather, we need to keep in mind that this promise is always there, always in the back of our heads, in our very own imperative to evolve through (self-)reflection, knowledge, and technology. Harari's Homo Deus is a prime example of how this promise is inserted into the public imagination, where it reproduces and evolves alongside technological possibilities and in concert with other representations.

This chapter set out to trace a trajectory from molecularization to plasticity and the desire for modification to the project character of contemporary embodiment. The view of the body in contemporary medicine as a complex network of composite parts - what I have termed a mechanistic-systematic view of the body - has allowed individuals to perceive of their bodies as objects that can be 'tinkered' with, while molecularization has extended this view into ever smaller and previously hidden regions of the body. But molecules, as the new objects of desire and subjects of intervention, signify more than

Marc Chrysanthou focuses on this idea of utopia, more precisely, the "healthy human body" as a new utopian project in postmodernity (469). I take up this idea of "utopia" in connection to the body. Broadly defined, utopia in this case denotes a future state of being that remains imaginative, always out of reach. The healthy human body, similarly, is utopian in that it is based on a (collective) imaginative, but impossible ideal of perfection. 
their biological function: they promise control and authority, certainty and potentiality, change and intervention, perfection and enhancement. But they also signify a new vulnerability, a new contingency of human life, based on risk calculations and preemptive interventions - enforcing a dogma of personal responsibility and self-determination. These promises and vulnerabilities are often, but not exclusively, made sense of in narrative and the imagination, in fictive worlds and imagined bodies. Those serve as tools to think through what we desire and fear, how we want to live, what kinds of social and political structures are possible and, crucially here, what kinds of bodies we want to possess. As such, they are one instance through which bodies are thought of as open projects; others include discourses of self-determination and identity construction, of beauty and health, of freedom and individuality. This is also where promise and plasticity come together: The promises created in cultural discourses give the impetus for change, while plasticity provides the material basis, on which in the era of self-management and self-responsibility individuals build their own body-projects in a truly selfdirected fashion.

If we talk about the body as a project, we talk about it as both a material body that is worked on and as an abstract process embedded in a larger 'project' we call self or life. We here, thus, do not just look at a physical project but also a much more epistemological question of how we approach and define humans, bodies, life. The view of the body as a project and the desire for transformation, perpetuated also by fictive imaginaries, will in their essence also have an impact in the context of DIY biology and medicine. "The ultimate DIY project becomes the body itself," writes Chrysanthou (471). DIY biology and medicine is not necessarily concerned with transformation, perfection or enhancement, but with their foundational ideas: It aims at shaping nature, tinkering with biology, carrying out self-directed interventions - to maintain a status quo, restore 'health' however defined or enhance our dispositions. Some of these ideals and cultural values behind DIY biology and medicine are the topic of the next chapter. 


\title{
4 With my Own two Hands - Tracing DIY from Individualism to Maker Culture
}

\author{
"As makers of today and shapers for to- \\ morrow, we Americans seem to share an \\ inborn understanding of how to go about \\ making the things we want." (Banes and Hoag, \\ American Maker, 2:40-2:50)
}

Most of the developments in the field of DIY are not entirely new shifts but slow developments in multiple social, cultural and technical areas. The idea of "emergence" can be a useful tool here: Rose uses "emergent" or "emergence" loosely to "refer to something new, which arises not from a single event of discovery, but often unexpectedly and contingently at the intersection of multiple pathways" "Genomic Susceptibility" 141). DIY, as practiced today, is the result of a sociocultural, political and indeed material context in which different ideals, rationales, discourses, but also technologies and practices blended: concepts of the individual as responsible, self-reliant, and self-managing; discourses of continuous progress and the perfectibility of life; ideas of bodies as in need of enhancement to achieve authenticity, wholeness and social status; a renewed focus on the material environment; the conceptualization of all aspects of life as a platform to be 'hacked'; the development of tools and techniques that allowed more people to participate in material projects of their own and to share and collaborate via open communication technologies; the privileging of participation, access and bottomup transformations. In this chapter I try to bring together these multiple, sometimes seemingly disparate, pathways.

The purpose of this chapter is to demonstrate the continuity of DIY, so that in the end I can show which facets of 'older' DIY practices can be carried over into 'newer' ones, especially in terms of critical positions but also regarding positive developments and tendencies. A historical reflection can illuminate where to look for parallels and problems as well as provide background for contemporary debates. This is particularly useful because considerations of older forms of modification or practices of do-it-yourself takes place with a certain degree of hindsight that can prove to be a valuable guide for present concerns. I will first ground the DIY techniques in American cultural values and ideologies, including but not restricted to individualism, a belief in progress, enhance- 
ment, and perfection. I then trace the rise of do-it-yourself as cultural practice and imperative, looking at its characteristics, historical contingencies, cultural purchase, and to some degree, transformative potential. I focus on the culture of DIY that has shaped the last century of American life - with varying intensity - and how its basic principles have spun much further, intersecting with and transforming other contexts such as science and the medical market. What is the reason for the proliferation of DIY also in areas unrelated to its 'home domain'? What are the repercussions of the empowerment and responsibilization of the individual perpetuated through the DIY ethos? What is the cultural and social significance of access to knowledge, of openness and transparency? These are but some of the questions that guide my discussion.

\subsection{From American Individualism to the Promise of Perfection}

Christina Lake, in her discussion of biotechnology and posthumanism in science fiction, identifies "largely unchallenged and invisible cultural assumptions" in advanced technological societies such as the US that propel biotechnology forward: She writes, “...what we have in contemporary America is a society of individuals who think that their bodies are essentially plastic, who think of their lives as a project, who look to technology to solve their problems, who value individual autonomy above most other things, and who are enculturated to believe that money can buy happiness (18)." Similarly, Peter Conrad claims that the "wellspring of biomedical enhancement is embedded in the very fabric of [American] society," among them a cultural belief in self-improvement, individual desires to outshine the competition, faith in technological solutions, and health care as a market economy (Medicalization 96). Some of these - plasticity, the body as project, scientism and technological determinism - I have mentioned in previous chapters. This subchapter now turns towards cultural ideals and concepts that shape both the trend towards 'doing-it-yourself' but also towards bodily modification and self-improvement more generally. The focus is on individualism and self-sufficiency, the Protestant work ethic and American Dream, ideals of progress and perfection, consumerism and health as a value.

While the cultural beliefs and values of white Europeans formed the template for current American (mainstream) culture (Hall 7), unique American experiences such as the frontier cemented some of these early values into the cultural consciousness and public memory. Even though American culture has transformed since then, some values and beliefs are still present today, such as individualism, competition, the Protestant work ethic, status and power, an emphasis on the scientific method, a belief in continuous improvement and progress (Hall 7,16$).{ }^{1}$ These are also foundational for the prevalent

This discussion, as I need to acknowledge, is rather simplified and generalized. Its aim is to sketch some of the core ideals and values that shape American culture on a collective level and that can be considered as foundational for the high cultural purchase of DIY practices and self-reliance. Individual values, of course, might differ greatly from those sketched here and there are two things that will be left out, primarily due to spatial considerations. First, the narrative sketched here often neglects minority cultures, their experience, struggles and influences on majority cultures. Second, its gendered dimensions only play a minor role: The story of the 'self-made man' is a pri- 
'do-it-yourself' ethos in American culture. 'Do-It-yourself,' thus, can be considered as quintessentially American, founded on some of its core values and ideals.

\section{"God Helps Those Who Help Themselves"}

The first value complex I want to consider is individualism, self-reliance and self-improvement. In the US, these three go back to the country's Christian heritage, beginning with the first settlers. It is at that time that ideas of the self and individual became central. The Protestant revolution, according to Rose, gave rise to a new "culture of the self" and systems of self-direction, in which individuals bear the responsibility for their own thoughts, choices, actions and failings, giving rise to new "technologies of the self" based on self-inspection, such as the Puritan practices of confessional, daily diary writing (Governing 224-26). Rose's account in Governing the Soul traces the move from external pressures to internalized norms of conduct and behavior, a self-government (of the soul or the body) that the self does herself out of her own free volition. Similarly, Irvin Wyllie and Richard Huber, in their 1954 and 1971 examinations of the self-made man and success in America, respectively, contend that the ideal of success - often in the form of accumulation of wealth - is tied to America's Christian heritage. Self-improvement's origins, according to Huber, are closely associated with a prevalent character ethic of moral purity and Christian values. Wyllie also highlights the early Christian origins of self-help so key to the myth of the self-made man. The belief in continuous improvement and progress is deeply engrained in the Protestant work ethic: the belief in hard work as an opportunity to achieve success and personal fulfillment (Hall 7, 16, 229-32). The narrative formula used in Horatio Alger's famous tales of morality - young boys escaping poverty, overcoming obstacles in their rise to economic success through hard work or good character to then lead lives of middle-class security and comfort - exemplifies this connection between (moral) purity, (economic) success and self-improvement as ideals of American life.

In her examination of self-help culture in the US Sandra Dolby writes that Americans have from the beginning felt a need to scrutinize and improve the self: "God expects us to seek to better ourselves, or so the roots of Puritanism would have us believe" (2021). The self-examination central to Puritan notions of the self, thus, can be seen as the very basis of ideals of self-improvement, ${ }^{2}$ while their focus on individual choice is one of the first cultural instances in which individuals are responsibilized for their own (spiritual) wellbeing. Self-responsibility for one's position in life and choices about available lifestyles are therefore some of the early values in the history of the new nation. According to Sacvan Bercovitch, this Puritan legacy is foundational for the very rhetoric of an American identity based on individualism, autonomy, and choice (ix). The expression "God helps those who help themselves," used by Benjamin Franklin in his Poor Richard's

marily male narrative, especially in the historical context that I line out. Intersectional considerations would probably reveal a wealth of new material and critique points, they would however unfortunately also go beyond the scope of this short excursion into the background of DIY. 
Almanack, fittingly summarizes those ideas of self-initiative and agency. ${ }^{3}$ They are also the basis for some of the foundational myths and stories of American culture, such as the 'Horatio Alger'-type stories of success, and 'bootstrap' ideologies like the American Dream.

Ideals such as autonomy, personal choice and control of one's fate - basic tenets of this early settler mentality - are also foundational for the preoccupation with individualism as another "American prerogative" (Hall 23). ${ }^{4}$ One of the (linguistic and cultural) core manifestations is the idea of 'rugged individualism..$^{5}$ This phrase, though older, gained widespread cultural currency during the Great Depression: US President Herbert Hoover (1929-1933) employed it to convey the idea that individuals should be able to help themselves and can succeed on their own, thereby justifying less involvement of the government in the economic life of the people. Hoover in his Oct. 22, 1928 speech in Madison Square Garden, New York, NY claims that the American system of "rugged individualism" is better for progress, economic prosperity, initiative and enterprise issues that for him reach into the "very roots of American life," connected to American ideals of self-government, liberty, freedom and opportunity. Individual initiative and individual enterprise for him are necessary for economic advancement, while increased government interference would halt the American march of progress and diminish the progressive spirit of the American people. ${ }^{6}$ This evocation of self-sufficiency and self-government as American core principles of course echoes earlier phases in American history, and are in fact uttered at a time when another ideal of American life was publicly addressed as foundational for American culture and character: the frontier. ${ }^{7}$

Franklin is often, wrongly, credited with the first English rendering of this phrase, which originated in Greek philosophies, fables and tragedies. It is nonetheless his use that gave it widespread currency in the US, turning it into an often-quoted phrase which many people seem to misperceive as written in the Bible.

4 Bercovitch, similarly, identifies self-examination and personal choice as central elements of Puritan conceptions of the self that further the early development of Western individualism (1-34).

5 According to Dolby, Alexis de Tocqueville's treatise on Democracy in America (1835) is one of the earliest works that addresses this idea of rugged individualism. In his study of American culture, de Tocqueville describes his perception of individualism as a distinctly American personality trait that has attained dominance over ideas of community (Tocqueville and J. P. Mayer). Dolby writes that, indeed, Americans have embraced this individualism and self-reliance since the foundation of the Republic and until now often value their individualism higher than the community (20-21). Fittingly, also Ralph Waldo Emerson in his "Self-Reliance" (1841) argues for spending more time reflecting on the self - in solitude away from the community and social obligations (Emerson, "Self-Reliance"). Robert Putnam's Bowling Alone (2000) is one of the seminal academic explorations into this duality of (increasing) individualism and (declining) sense of community.

6 What needs to be recognized, following this historical trajectory, is that more liberal political campaigns, such as Franklin D. Roosevelt's "New Deal" (1933-1938) or Lyndon B. Johnson's "Creat Society" (1964-1969), might point to a shift in values towards community orientation and social security. However, even though these 'liberal' decades have changed the political landscape of the US, and gave birth to state-guided social security measures in place until today, overall - as Cowie and Salvatore argue - the New Deal could not fundamentally transform the individualistic (political) culture and society towards a welfare state. 
From the early settlers until the 'closing' of the frontier towards the $20^{\text {th }}$ century, it had occupied a special place in the image or imagination of America at home and abroad. The vast land invited its new inhabitants to explore and claim uncharted territory in search of individual success and economic well-being. ${ }^{8}$ In America, the legend went, you can shape your own destiny, build a new life, take control, reach wealth and success, you can search for a better life. This foundational myth of American culture is also emblematic of American ideals of progress. According to Paul O'Neil, in The End and the Myth, self-reliance and inventiveness are some of the foundational outcomes of the frontier that have shaped American character and culture. Perpetuated again and again in the cultural imaginary, this 'unique' and shaping experience became part of American collective memory.

Individualism, self-responsibility and self-reliance are thus some of the foundational ideals of American life. There is a pervasive notion that people are self-made; it is a culture that promises individuals limitless possibilities of self-realization (cf. Fluck 29). The self can be changed, but it should be in a self-directed manner, stressing independence and personal responsibility for one's life choices (cf. Hall 23-32). This core American myth of the 'self-made' man - whose epitome Alger's protagonists were and whose roots have so thoroughly been studied in American history (cf. Wyllie, Weiss) - is visible until this day in discourses of self-awareness, self-confidence, self-control, selfdiscovery, self-fulfillment, self-determination, self-help, self-improvement, self-optimization, and other "self"-compounds, as M. Butler finds ("Spiel" 78-80).

\section{Progress, Perfection, Enhancement}

This discussion of foundational American values and myths also points to the utopian core of American culture and society. Paul Auster, in City of Glass, puts the following words into the thoughts of his protagonist: "From the very beginning, according to Stillman, the discovery of the New World was the quickening impulse of utopian thought, the spark that gave hope to the perfectibility of human life..." (71). From its beginning, America was built on the idea that a better life is possible if you are willing to work and sacrifice for it. Or, as Herbert Croly wrote in his 1909 treatise on The Promise of American Life, in the imagination of its citizens America is the "Land of Promise:" "From the beginning Americans have been anticipating and projecting a better future. From the beginning the Land of Democracy has been figured as the Land of Promise." Since its early days America was connected to an idea of progress, the promise of a better future, individually and collectively, but this promise is not just an anticipation but also a responsibility: "It becomes in that case a responsibility, which requires for its fulfillment a certain kind of behavior on the part of himself and his fellow-Americans. And when we attempt to define the Promise of American life, we are obliged, also, to describe the kind of behavior which the fulfillment of the Promise demands." (Croly 3-4) As with the

Of course, the highly problematic nature of this type of conquest needs to be acknowledge. European settler's intrusion on Native American lands was not only often fatal for Native Americans but also took away their innate claims to the land, resulting in centuries-long violence, oppression and discrimination. 
Hoover speech above, mentioning this ideal is not meant to start a discussion of the political debates and actions such ideas sparked but rather to show how deeply embedded such ideas are in the American collective memory and imaginary. Perfection and enhancement flow naturally from the basic ideals of individualism, autonomy, self-reliance, unwavering progress so ingrained in American culture.

Another (literary) example of the deep embeddedness of such ideals of perfection and (body) modification is Nathaniel Hawthorne's short story "The Birthmark" (1843). Full of almost magical scientific experiments, Hawthorne tells the story of a scientist who obsessively tries to 'cure' the birthmark of his otherwise beautiful, perfect wife, leading in the end to her untimely demise. ${ }^{9}$ Soon after their marriage Aylmer, "a man of science," becomes increasingly irritated by a birthmark in the shape of a tiny hand that adorns his wife Georgiana's cheek. For him, this birthmark becomes a symbol of sin, decay, death, sorrow, imperfection, a "frightful object, causing him more trouble and horror" than her beauty ever caused him joy. Determined to remove the mark from her cheek, it becomes the central point of their life together, turning it into a burden also for Georgiana. She had previously seen it as a magic fairy's touch but now wishes it removed at all cost, simply to satisfy her husband and free herself from his gaze. 'Curing' her of this physical imperfection, however, is a death sentence for Georgiana. It is implied that she is now too perfect a creature to live. This $19^{\text {th }}$ century story speaks to the cultural embeddedness of the fascination with (self-)enhancement, of making the already good "better than perfect." 10

Even natural limits have never been seen as a deterrent: As Ralph Waldo Emerson already remarked in 1836, the American attitude to nature is that of a refusal of "natural" limitations, that nature is "not fixed but fluid," can be altered, molded, made ("Nature 1836" 80). As Lake summarizes: a widespread attitude is that Americans must "build their own worlds" and shape their lives according to their desires (1-2). Nature becomes a resource that Americans can use, for example to further their own success or to transform their body according to social and cultural ideals of "beauty, youth, and longevity," as Jakob J. Tanner claims (40). A core belief is that people can shape themselves, be whatever they want to be, and do what they want to do - and that technology can help in overcoming natural limitations (cf. Lake 13). This belief is also the basis for the belief in science and technology as means to shape and define the self.

We can argue that part of the foundation of American culture and society, is a belief in the perfectibility of human beings through work on the self. Such work to optimize the self is common, encouraged, sometimes even demanded: What previously was primarily expressed as work on personal morals or an abstract sense of self, is in the mid-20 th and $21^{\text {st }}$ centuries reframed as (also) a materialist work on the body. In

9 Hawthorne, it seems, could only image the science in his book in analogy to magic.

10 In Wegenstein 's reading of the short story, it is not just a "warning' not to mess with nature, it is also an exploration of the meaning of beauty and our attempts to define and capture it." According to her, the story also speaks to some of the anxieties and problems around the process of makeover, such as the desire to restore or reach some "image of perfection" that might not necessarily be self-chosen and the importance of the gaze for the desired outcome of the project as well as its effects on the object of improvement: When the birthmark is turned into a problem by Aylmer, his wife is thrown into misery and self-hate (Cosmetic Gaze 70-72). 
such a discourse the body is not a given but, as Wegenstein terms it, "a platform to invest in." In such a "make-over" culture of fluid selves and bodies, the body becomes a project that is to be aligned with your own individual life project (Cosmetic Gaze 131). Self-control, self-discipline, self-denial and will power - note the ongoing focus on the self as agentic and responsible - are needed to perfect and govern the body, most visible in discourses of diet and physical fitness that require deliberate action on the body and serious commitments of energy and time to the cause (Lupton, Medicine 42-43). Fitter bodies, a youthful appearance, or athletic vigor are all "deeply ingrained social and individual goals in American culture" (Conrad, Medicalization 89). Such an ideal of perfection, that is also perpetuated in fantasies of perfected bodies, opens up the body to enhancements and modifications. Strategies and techniques of enhancement - ranging from supplements, training regimes, to after-school tutoring - are today enriched by biomedical enhancements through medical interventions aimed at bodily improvement such as cosmetic surgery, performance-enhancing drugs, or "cosmetic psychopharmacology" (Conrad, Medicalization 70-71). Biotechnological developments and the contemporary mechanistic-systematic body concept mean that also the inner makeup of the body is amenable to enhancements. The plastic body is open for modifications that reach deep beyond the skin.

In his exploration of American Medicine and the American Dream, Better Than Well, Carl Elliott locates the paradoxical uneasiness about and appeal of enhancement technologies in the American understanding of the self. He argues that in the US individuals are encouraged to express what makes them who they really are and to present themselves in the best light possible (70). The importance of and responsibility for individual choices and "life projects," according to Elliott, explain the "lure" of many enhancements technologies as tools to make said life project better, more successful, more rewarding, more "fulfilled" (299). This cultural ideal of "fulfillment" as the essence of a "good" life makes individuals susceptible to the idea that they and their life need to be better, can be improved (289-99). ${ }^{11}$ This ideal of self-fulfillment ideally means creating a more authentic self, and presenting that self and its social status to others. The American belief in technical solutions - mentioned already above - means that also biomedical and technological enhancement techniques are fair means in this game of presentation, judgement and the pursuit of a good life (C. Elliott $\mathrm{xx}-\mathrm{xxi}) .{ }^{12} \mathrm{~A}$ cultural ideal is given expression through biotechnological processes. Here, we can see how ideas of self-modification are also connected to the pervasive belief in technological and scientific solutions perpetuated by scientism as a dominating world view (cf. Ch. 2). By trying to connect the acceptance or rejection of biomedical technologies to some of the foundational American "promises" such as fulfillment, happiness, choice, regard for the individual, Elliott's "diagnosis" (as he calls it), connects the technologies of to-

11 'Fulfillment' is a highly individualistic and subjective notion that nonetheless is floated around as a 'cultural ideal' with no clear connotation. Who then is to judge what a fulfilled life looks like?

12 Carl Elliott writes that Americans see the pursuit of self-fulfillment and happiness as a "strange sort of duty," as an obligation to be happy, an obligation to the self (303). This stance seems to mirror Croly's assertion that the promise of America responsibilizes Americans to certain behaviors. 
day to centuries old traditions and values. He thereby elucidates how these values are the foundation of contemporary uses of biomedicine.

Perfection, however, is a moving target, something that is always 'not-quite-thereyet,' a promise that notoriously remains unfulfilled. That we can never reach it lies in its very nature. Whenever one ideal of perfection is almost achieved, we tend to find new flaws, question the ideal, find a new goal, a new subject of improvement. This is particularly relevant in a culture that continuously craves bigger, better, faster, stronger. At the same time, it buys into almost modernist grand narratives of progress: We need to push the boundaries of our bodies and technology, because only progress will bring us closer to that which we desire. This promise of progress, perfection and fulfillment, particularly in US culture, is reinforced by consumerism.

\section{Consumerism and the Value of Health}

Consumption and commodification, according to Paul Brodwin, have to some extent always been central to personhood in the US, especially in the $20^{\text {th }}$ century (15). Consumption is seen as a means to perform a certain identity, assert one's uniqueness and individuality. Consumerism, Hallam Stevens argues, is part of the American Dream and the quest for happiness, the idea is that "you can spend your way to happiness" (275). The "postmodern identity-seeker," a type of consumer that Colin Campbell describes as being prevalent today, consciously consumes products, selecting them for their symbolic meaning, in order to present a certain identity, impression or lifestyle (24). Consumption, thus, is also part of the construction and presentation of cultural, social and today increasingly also bodily capital - and it is this connection between consumerism and the body or health that I want to look at.

During the $20^{\text {th }}$ century patients have become reframed as consumers that play an active role in the medical market place, whose choices and desires structure supply and demand, whose preferences sometimes even take precedence over the authority of medical professionals (Conrad, Medicalization 151-55). The beauty industry, or more precisely cosmetic surgeries, are a common example of how patients' desires influence bodily interventions (cf. A. Elliott; Wegenstein, Cosmetic Gaze 114). As seemingly autonomous participants in the medical market, the patient-consumer has to assess and select how much they can or are willing to pay for 'health' or wellbeing as the newest consumer good (Lemke, "Disposition" 559). Exercising their right as a consumer, as Rose already hinted at, however, is reserved for those who have financial and cultural resources: Gaining access to technologies of maximization requires capital and it is the people who have that sort of capital who drive the quest for enhancement (Politics of Life 105). As Lupton remarks, informed consumer choices are often closer connected to middle class economic ideologies (cf. Medicine 149). Capital, prices, and economic class are thus creating barriers to access. Health becomes a commodity; you have to decide yourself how much of it you can afford for which price.

As a consequence of a consumerist ideology of the self and wellbeing, health receives a price tag. It becomes a value in a double sense: It is valued as a state-of-being and it has a literal economic value as a resource that people use to position themselves as fit, exploitable, 'value-adding' in a market economy. As a commodity, health and enhance- 
ments can be purchased in the medical market place. However, this commodification also means that we need to invest into our bodies in order to reap the benefits: we need to invest work, time and money so that in the end we (seemingly) have added value in terms of bodily and social capital. The body is seen as a 'raw material' that has to be worked with. We spend hours perfecting our bodies, appearances, and (virtual) persona so that in end other people recognize the work we have put in (value it), but by doing so we also make our bodies more in line with neoliberal market ideals. Similar to antique techniques of the self - a "care for the self" closely connected to philosophical ideas of a "good life" - today self-care has become an ethical maxim and moral requirement (M. Butler, "Spiel" 77-78). ${ }^{13}$ Health, wellness, well-being, fitness are values pioneered also by "cultural heroes" such as celebrities or more contemporarily, social media personalities, health and fitness bloggers, that create artificial needs and trends. Health and body-conscious behavior are engineered also by popular culture. ${ }^{14}$ But the goal, as stated above, is no longer simply health, but more than that, the moving target of perfection, the promise of completeness and authenticity.

To summarize the basic ideals taken from this discussion: There is a keen focus on the able, autonomous and responsible self in American culture, an individual that is expected to take care of its own situation in life, that is self-reliant and self-governed, coupled with an idea that there is always room for improvement or perfection. The latter is emblematic of contemporary developments in relation to health and wellbeing, while the former is especially relevant for the notion of 'do-it-yourself.'

\subsection{Tracing DIY - From Home Improvement to Citizen Science}

This subchapter wants to go back in history to connect contemporary forms of biological DIY to their older precursors: It looks at the history of DIY from home improvements to leisure activities, often bordering on the thin line between bare necessity and beloved hobby. It thereby tries to follow the successful rise of DIY as an omnipresent cultural trend that reaches into almost all aspects of human life. I then move on to more recent developments in the DIY area. Topics include the rise and success of maker culture, hacking and technology as well as the transformative and democratizing potentials of self-made solutions. Lastly, I turn my focus onto amateur and citizen science and its new guise: DIY biology.

As the name already suggests 'do-it-yourself' implies active individuals 'doing' projects of their own choosing themselves, rather than looking to others for their

13 Historically, a prime example is the hygiene discourse of the $19^{\text {th }}$ century, that as Philip Sarasin argues in Reizbare Maschinen, can also be seen as a set of instructions for a "care for the self" (2324), constructing a modern body of health and well-being - an example for how a new popular science found expression in material work on bodies.

14 John McKinlay looks at how at-risk behavior in the $20^{\text {th }}$ century was engineered and artificial needs created, by "piggybacking" them on legitimate values, beliefs and norms, often through the endorsement through "cultural heroes" (580). One of his examples is how smoking tobacco was championed in the public. Today, these same techniques are used to instill health as a cultural value. 
completion. These projects can vary widely from cooking to gardening, knitting, crocheting, sewing, woodwork, pottery, to hacking, science, and biology. Paul Atkinson summarizes that DIY has in past scholarship been approached as craft activity (Attfield), pastime (Gelber), financial necessity (Goldstein), lifestyle choice (Sparke), as social and cultural phenomenon, from economic, ideological, and feminist perspectives (1). The multiplicity of contexts in which 'DIY' has been described also reflects one of its essential complexities: There is no clear-cut definition or classification of what constitutes DIY, which practices are included, which should be excluded.

According to Atkinson, the term "DIY" is employed for a "wide range of activities which are carried out for a variety of reasons," though he does split them into the "making of objects" - including handcrafts, furniture building, sewing, knitting, and the like - and home maintenance, such as decorations, gardening, building, or renovations (2). DIY can thus be a hobby and handcraft, essential home maintenance, or lifestyle and consumer activity. However, as David Gauntlett describes, these practices often overlap in their motivation and ethos, namely that you can do it yourself and it feels good to do so (56). This notion of empowerment is the joint mentality of many DIY activities. Also Atkinson takes the motivation behind the activities as a key organizing principle, categorizing them into four different types of DIY: pro-active DIY (those self-directed activities that involve creativity, skill, originality in which personal pleasure or financial gain are key motivations), reactive DIY (hobby, handcraft, building activities with kits, patterns and predetermined components, with pleasure and occupation in one's free time as motivation), essential DIY (home maintenance activities out of economic necessity), lifestyle DIY (home improvement as consumption) (2-3). These different types serve as a broad classification of DIY activities that also show how complex the motivational forces behind DIY are. But they can also be seen as reflections of different phases of DIY during the past century, moving from essential, reactive activities to lifestyle and pro-active ones.

\section{DIY Conquers Culture}

We can roughly discern three, to a degree intersecting waves of DIY in the last century - the 1950 s/60s home improvements, $80 / 90$ s punk counter culture, and the more contemporary 'maker movement.' These waves represent instances in which DIY cropped up as an explicit trend and start with the first time that the term 'do-it-yourself' was used as a denominator. Its history, however, is much older, when self-made solutions were necessary and normal parts of everyday life.

Historically, DIY has always straddled an ambiguous position between pastime and necessity. ${ }^{15}$ In the $19^{\text {th }}$ century, DIY activities were unavoidable for most of the population from the poor to the middle classes. The upper classes, on the other hand, saw arts and crafts as a good way to pass time doing something useful, such as knitting, stitching, and so forth, and taught them as signs of (female) accomplishment. This early

15 I broadly combine Atkinson's narrative of the historical development of DIY primarily in the UK with Gauntlett's considerations of the 'American' spin on DIY. 
arts and crafts movement, as Gauntlett claims, "connected meaningfully with American notions of self-reliance, individualism, community," but the American version of this movement gave it an American spin by adding a "democratic element" to DIY culture (47-49). Gauntlett's example is Gustav Stickley, who published the magazine The Craftsman from 1901 to 1916 and through this medium spread a "distinct American version" (49) of the arts and crafts movement. Stickley, Gauntlett argues, had a vision of "'a simple, democratic art' that would provide Americans with 'material surroundings conducive to plain living and high thinking" (49). Subverting his own business of selling finished products, he inserted plans for needlework, furniture building and metalwork in his magazine. This early "open source" system, claims Gauntlett, is the epitome of an (American) anti-elitist, "democratic approach" to DIY (47-49).

During the Great Depression and primarily the Second World War, then, in the UK and to a lesser degree the US a "make do and mend" ethic made DIY activities a necessity of national concern that was propagated as a valuable skill and crucial contribution to the war effort on the "home front" (Atkinson 3-4; Gauntlett 61). This reality of DIY as an economic necessity should not remain unrecognized. While this characteristic is part of some later uses of the term, the majority of DIY activities quickly moved into the realm of hobby and voluntary activity. Atkinson references Carolyn Goldstein when he writes that in the US DIY became "perhaps less of a necessity and more about social aspiration" and the realization of new domestic dreams propagated by the rise of suburban living (Atkinson 3-4). The Second World War, as Atkinson claims, had prepared especially the men with the skills to now tackle the projects of the day: Home improvements.

In the 1950 s and 60s these self-made home improvements became somewhat of a national obsession, representing the first time, according to Steven Gelber, that 'doit-yourself' entered public conversation on a large scale. ${ }^{16}$ In 1952 the magazine Business Week proclaimed the 1950 s to be "The age of do-it-yourself" (271). Gelber maintains that even though the phrase had been used before, the proliferation of the term "do-it-yourself" in the 1950s was the one that made it "commonplace" and gave it its widespread currency (271). DIY was mass-marketed and popularized via instructions in newspapers and magazines but also expositions and workshops directed at the new DIYers (cf. Atkinson 2).

In essence, much of this first large-scale wave of DIY consisted of work around the house, be it decorations, renovations, or improvements - turning homes into hobbies (Gelber 271). With the rise of suburbia as the American ideal, remodeling and improving the cookie-cutter homes that surfaced all around the country became a (primarily middle class) homeowner preoccupation. Families banded together to paint walls, remodel basements, attics and garages, upholster furniture. They gave their home an individual touch and personalized it, with their own two hands, effectively creating a new way to productively pass time together. A prerequisite for this development was the new availability and affordability of power tools designed for lay users, such as power drills or woodwork tools (Gelber 278-82). This 'new' trend was a mixture of necessity and lifestyle DIY, a necessary means of home maintenance but also leisure activity and consumer 
choice - or as Gelber calls it a form of "productive leisure:" A work-like activity that is however carried out because people want to not because they have to (269).

These DIY activities seem to embody the very definition of a "hobby:" a solo or group project done during spare time, "reinforcing and replicating work values" (providing psychological fulfillment), with the extra "benefit of being useful" (Gelber 269). However, they quickly also became somewhat of an obligation, especially for men. Gelber's analysis shows how DIY became not a voluntary but a mandatory hobby: Men were expected to take up the role of handyman if they did not want to lose their standing in the community; DIY became a "badge of "manhood" and its tools (once mastered) emblems of a new masculinity $(287,290,294) .{ }^{17}$ Another downside along gendered lines was that while the whole family seemed to be included in the projects, there nonetheless was a distinction between tasks for men, primarily those including heavy machinery and hard manual labor, and women, who were primarily expected to take on the 'softer' and supporting tasks such as planning, cooking, or decorations. In DIY exhibitions and magazines craft-and decoration-oriented projects were marketed for women as well. ${ }^{18}$

At the same time, the de-skilling of the tasks through assembly sets and kits, heralded the invasion of consumer culture into DIY: New products that were easier to use were designed and new forms of distribution implemented that would better satisfy the growing consumer demand to take part in this new suburban-American ideal - pre-cut wall papers and fast-drying wall paints are cases in point (cf. Atkinson 4-5; Gelber 29899). DIY was recognized and exploited as a major new market, that according to Mark Hatch in 2014 was worth $\$ 700$ billion in the US DIY home improvement industry alone (12). Self-assembly and pre-fabricated kits became necessary means to fully permeate this new market, opening up the experience of DIY to all ages and levels of skill. ${ }^{19}$ This downright assimilation into consumer culture as well as the problematic gender relations and prescriptive tendencies are some of the more crucial downsides of DIYs first wave.

The compensation came in the 1980s and 90s when DIY was revived as part of the punk, protest and counter cultural movement. After a time of increasing commodification of culture, DIY now was seen as a means to counter that commodification. The "lo-fi" movement in punk and feminist cultures saw the birth of low-cost, DIY alternatives to mainstream media, meant also as forms of activism and protest: Most popular

There was an Australian TV show called Handyman that aired between 1957 and 1958. This genre has remained popular until today, reaching from the TV sitcom Home Improvement (1991-1999) to a plethora of home renovation and home make-over reality TV shows. supporting role of women changed to make their input much more explicit (Coldstein 67-82, Atkinson 7).

19 cf. also Gelber on the history of kits. He claims that during the same time as the DIY movement in the 1950 s and 60s, kits and plastic materials that had previously been used to assist the rehabilitation of soldiers wounded during World War II became a civilian product that reduced the "productive process" into the assembly of pre-fabricated parts. Nonetheless, Gelber argues that together with DIY home improvements it brought handcrafts and making activities to an exceptionally wide audience, allowing parents and children to feel the satisfaction of having "done something productive with their hands" without however having to learn a new craft skill (298-99). 
among them were "fanzines," DIY productions of fan magazines that came with a distinct aesthetic of cut-and-paste pastiche and collage, appropriating and critiquing mass culture at the same time (Ratto and Boler 10; Triggs). "Craftivism" is a more contemporary equivalent of this protest-oriented, anti-consumerist DIY (Ratto and Boler 10). ${ }^{20}$ Craftivism or critical making combines DIY approaches with social activism and societal relevance (Richterich 160).

Since that time, such material renditions of DIY quickly spread and became a cultural phenomenon that has proliferated also in other areas: Making one's own clothing and accessories, DIY decorations and life hacks have become common and desired free-time activities. In the last decades, DIY has expanded even further with the global spread of the internet, where online tutorials, videos, and homepages dedicated to selfmade 'things' and easy DIY 'life hacks' can be found aplenty. The "diyization of modern society" as Buck Clifford Rosenberg called it in his examination of IKEA's self-assembly furniture - a new rendition of domestic DIY emblematic of postmodern consumer culture, he claims - has now reached almost all aspects of society. Today, rather than straddling an ambiguous position between pastime and necessity, however, it is wedged between consumerism and anti-consumerist activities.

\section{A New Quality of Making}

The latest rendition of the DIY ethos has spread under a new name: the Maker Movement. The underlying values, on the other hand, are still the same. As Sara Tocchetti comments, self-sufficiency as a (Protestant) American core principle is still one of its main sources. Fun, innovation, creativity, curiosity, collectivity, community, openness, excitement, passion, innovation - these are some of the most common buzzwords used by members of the community to describe this new movement, a self-proclaimed 'revolution' of how we design and produce, collaborate and create meaning. Like past DIY practices, the "maker movement" includes a plethora of different activities - from crafting to high-tech electronics - but with two decisive differences, as 'maker' Chris Anderson remarks: digital tools for design and fabrication and a new sharing ethos facilitated by the internet as a source of inspiration, community and communication. It is especially the "Web's culture and collaboration," Anderson claims, that is responsible for the new and unprecedented quality and scale of DIY (21). Mark Hatch, another "cultural hero' of the maker movement, similarly sees "cheap, powerful, and easy-to-use tools," easier access to knowledge, markets, and capital, a "renewed focus on community and local resources" as well as a desire for authentic things as the most salient trends that push the interest in making things and the maker movement (5). ${ }^{21}$

20 Spencer, Amy. DIY: The Rise of Lo-Fi Culture (2008) (historical overview); Levine and Heimerl. Handmade Nation: The Rise of DIY Art, Craft, and Design (2008) and Tapper and Zucker's Craft Activism: People, Ideas, and Projects from the New Community of Handmade and How You Can Join In (2011) focus on craftivism.

21 In my discussion I also include pop-cultural non-fiction written by members of the maker movement. They serve as illuminations of the values and ideals of maker culture, but also of how makers want themselves to be seen and of how the community sets its purposes and boundaries. 
They are not alone in their assessment: Critics that are not economically connected to the movement distinguish similar characteristics as giving the maker movement a new quality. David Gauntlett for example claims that collaborative, creative activities are on the rise both on the Web and in 'real life,' with the former being a great communication, networking and organization tool for the latter that shapes how maker culture is spread and conceptualized (11-13). ${ }^{22}$ Similarly, Alessandro Delfanti asserts that the DIY movement is "witnessing a renewal and is now part of a broader social phenomenon centred around the convergence between online peer production, the diffusion of cheap and open source tools and machinery (such as 3D printers) and a widespread 'maker' culture" (Biohackers 118-19). As critics and makers say alike, much of this new rise of DIY is due to the internet allowing new forms of communication and sharing with its collective and collaborative premise, thus fostering a democratization of and easier access to information. Gauntlett sees the internet as a new avenue of public participation, with the contemporary "Web 2.0" approach turning it into a decisively collective, collaborative, and shared space (3-5). Similarly, Mark Frauenfelder says the Web helped in the rise of contemporary DIY activities due to its greater "information-delivery capability" (15).

Not just the internet, but also the hacker culture of the late $20^{\text {th }}$ century had an enormous impact on contemporary making. ${ }^{23}$ Born in the 1950 s and 60 s and expanding alongside the development of the personal computer and internet as well as the counter cultural movements, hacking is a diverse phenomenon - its practices ranging from coding free software to breaking into systems and stealing data - with as diverse politics (Delfanti, Biohackers 56-57). ${ }^{24}$ However, the "hacker ethic" of those first computer hackers at MIT remains very much foundational for contemporary hacker culture. According to Steven Levy this ethic includes free and unlimited access to computers and information, a mistrust of authorities, a valuation of the quality of hacks rather than social or class criteria, and the realization that computers can have life-changing effects (Delfanti, Biohackers 58; Levy). Contemporary hacker ethic can thus be understood as a set of values and core ideals, among them an open-source ideology, stressing openness, access and free sharing of information and knowledge; inclusion, collective work and peer recognition; innovation, curiosity, fun and play; as well as transparency and a distrust of institutions (Delfanti, Biohackers 3, 56-57, 138). The "essential meaning and promise of hacking," as Soderberg and Delfanti argue, is a "recuperation from below" in which technology is re-designed and created in order to serve the ends of those who use it (2). It is a grass roots initiative that aims to change the logics of contemporary production by focusing on those which will in the end be using the products. These ideals have shaped the outlook of makers. While makers might see 'hacking' as an immaterial form

22 On the web, he finds a broad range of collaborative activities ranging from Wikipedia, to political activism, YouTube and blogs; in 'real life' examples for him include crafts, clubs, DIY, re-using and re-cycling (Gauntlett 11-13)

23 Especially Alessandro Delfanti has looked at the hacker movement as foundational for the maker, and especially DIY biology, movement.

24 In fact, as Delfanti points out its political heterogeneity, diversity and multiplicity makes it so interesting and is, as he claims, reflected also in biohacking, which I come to later (Biohackers 12). 
of DIY and thus different from contemporary making, the values behind hacking are nonetheless certainly foundational for 'making,' and some of the (legal) goals of hacking are often taken as a blueprint for more material forms (cf. C. Anderson). Moreover, many makers have their roots in digital contexts and also linguistically there is an overlap. According to Steve Mann, making involves the moral, ethical, lawful parts of the "hacker ethos," combining the DIY ethos of earlier practices with the "do-it-together" (DIT) ethos of hacking (30).

However, in all its similarities to hacker culture, makers often consciously pitch bits versus atoms, software versus hardware, firmly positioning themselves on the side of atoms (C. Anderson 14). Making is intensely material and differentiates itself through its materiality (cf. Bean and Rosner, Mann 30, Hatch, C. Anderson). The tactility and material presence of the objects made is one of the main selling points of the maker movement, thereby positioning itself as an alternative to the increasing virtualization, abstraction and displacement of contemporary life. ${ }^{25}$ 'Making' according to its proponents, can counter the disconnection and alienation of late capitalism: making is intensely personal, with many contributors such as Anderson or Frauenfelder arguing for the transformative potential of making on the individual. 'Makers' are embedded in what they make and draw from this a deep satisfaction and connection to their environment, they claim. Hatch proposes that making will allow you to feel whole, a "more complete version" of yourself (1-2). With this high emphasis on personal change through the fundamental experience of making, Hatch positions making as similar to other ideologies of self-transformation, "techniques of the self" (Foucault) fundamental to the experience of being human. While I think this view might be a bit tainted by his entanglement in the maker movement, the idea of 'making' as a form of (psychological) self-transformation, its materiality and deep personal connection nonetheless align with my argument about the connection between DIY and self-modification.

Those describing the maker movement from within often tend to focus on the "broader social changes their practice promises to bring," as Bean and Rosner argue. They focus not on the material consequences but on social change, often in the language of "revolution" (26). While revolution might be exaggerated, my hypothesis is that DIY or making is a cultural, as well as material, social and political movement with tremendous potential for change. This is corroborated both from within and without the movement. The revolution makers like Chris Anderson envision is one that mirrors the development of web-based start-up companies (C. Anderson; Hatch): Innovation, economic considerations, new impulses for entrepreneurship and manufacturing based on open source and open innovation approaches form the core of making's

25 This materiality, the 'genuine' nature of the end product, (re-)connecting both with where things come from and how they are made, certainly, is one of the many appeals of making that critics and makers have cited. Other appeals include: individuality (Atkinson 7; Celber 292); agency and competence (cf. Gauntlett 2); play, experimentation, tinkering (cf. Diana); its process- and projectoriented nature (cf. Gauntlett 64-66; 95-106; Watson and Shove 81-84); community (Gauntlett 95, Ratto and Boler 8, C. Anderson); searching for and finding meaning (Frauenfelder); as well as desires and feelings of satisfaction and pride (cf. Gauntlett, Hatch). 
transformative potential for them. ${ }^{26}$ Others stress the possibility for community and collaboration, the emphasis on sharing and the interplay between individual and community (Delgado 66; Anderson 74). A democratizing potential is often ascribed to the new ideology of access: putting tools and knowledge into the hands of everyone who wants to use them is a necessity for the maker movement (C. Anderson 63). ${ }^{27} \mathrm{It}$ thereby creates more participation and also serves educational goals. Similarly, critics Soderberg and Delfanti describe making as a special case of citizen engagement that, despite the lack of a unifying collective identity or political subject, has been ascribe an "emancipatory potential" by many scholars (2). As such, DIY or making can also be political. Making is positioned as liberating individuals from the economic reality of today with its constrained and inflexible roles of production and consumption of goods and knowledge. It gives rather than takes away agency, provides an alternative and creative means to manage problems and find solutions. Alexandra Bal et al. argue that " $[\mathrm{m}]$ aker culture has the potential to represent a cultural model that encourages individual citizens to construct their own social realities, connections, and material aspects of living, to take user-generated production as a key to individual self-determination, knowledge sharing, and community building" (158) - an instance in which culture is made that is reminiscent of earlier times, as the discussion in the previous subchapter has shown. All of these potentials have their merit; which ones of them will be fulfilled at this time remains an open question. What we can ascertain is that DIY has become a social and cultural phenomenon, almost a cultural value in and of itself. Today, do-it-yourself is not merely a side issue but a pervasive principle of much of everyday activity, whose appeal lies in the cultural contexts of today as much as in its historical continuities.

\section{A Special Case of Making: Citizen Science and DIYBio}

This social and cultural trend towards doing things 'with my own two hands' spills over into more and more areas, among them DIY science and biology. But truly, citizen engagement in science is much older than that. Already Thomas Hobbes saw science as something everyone can do (Erickson 105). In contrast to the contemporary view of

26 Like the internet before it, Chris Anderson claims that "making" has the potential to give new impulses for transformations in manufacturing and entrepreneurship based on open-source ideals. "Making," for him, creates new forms of open innovation, new types of localized global businesses and entrepreneurship, creating new jobs and manufacturing possibilities in a world that is characterized by global outsourcing to cheaper production places. Focused on this entrepreneurial side, the "revolution" he envisions is one that mirrors the development of the Internet and web-based start-up companies. While Hatch and Anderson mention the intrinsic motivation and potential of making ("a better version of you"), they are very much focused on the social-economic level and makings potential for innovation and production.

27 Even though people like Chris Anderson often prefer to conceptualize the maker movement as a "garage phenomenon" with bottom-up approaches (Makers 20-22), Bean and Rosner, point out that this "maker revolution" in reality primarily attracts males with disposable (and thus higher) income, resulting in a high gender and class gap. But rather than broadening the base, Bean and Rosner contend, Make journal for example started a "sister journal" called Craft, focusing on "female" making (Bean and Rosner 26-27). 
the 'ivory tower' citizen engagement in science had actually been the norm for quite some time: From the very beginning of scientific knowledge production, 'citizens,' 'amateurs' or 'volunteers' had been involved in the process, from natural history, astronomy and meteorology, to epidemiology. ${ }^{28}$ Amateurs were collecting and classifying biological specimen, monitoring and describing the stars and solar system. Benjamin Franklin and Thomas Edison are probably two of the most famous amateur scientists and inventors in the American cultural imagination and 'founding fathers' of the 'inventor myth,' the lone genius tinkering his way to knowledge and innovation, prevalent until today. But also in medicine self-experimentation had been a major part of medical history before large scale and systematic clinical trials (Neff and Nafus 16). ${ }^{29}$

Media representations are symbolic of this pervasiveness. Magazines such as Popular Science or Scientific American published columns, articles and instructions well into the 1960s, detailing a wide variety of projects the public could tackle, from constructing electronics, to making amino acids, to experiments with cockroaches or growing HeLa cells in culture (Frauenfelder 188-89). In the last decades of the $20^{\text {th }}$ century, coverage was, according to Frauenfelder, slowly ceasing, shifting to 'Big Bio' and institutionalized science, while interested people jumped onto the next big thing: computers and software. Science-as-hobby, he proclaims, was dead - until about 2005 and the foundation of groups like "DIYbio."

Foundational for these groups was a disillusionment with Big Bio and corporate science, the exclusion of lay people from scientific knowledge, the spread of the open source and open science movement and a political request for public participation. The Open Science Movement advocates values such as openness, sharing, access and transparency, which would make science "more productive, more inclusive, more democratic," as many proponents claim (Delfanti, Biohackers 11). The goal is to "tear down the barriers to the access of scientific knowledge" (Delfanti, Biohackers 5), it is thus primarily concerned with the control of and access to information, public participation and restrictions to said participation. Open Science, like open source, denounces elitists and exclusive claims, while promoting open, peer-to-peer knowledge production (Delfanti, "Users and Peers" 1). Citizen science and P2P science is the result of the convergence of these phenomena with a broader availability of the needed technical infrastructure, the spread of maker culture with its support of free soft- and hardware and tools for online peer production as well as broader political shifts toward more participation and inclusive communication. As a result, Delfanti argues, today far more people are involved in the production and discussion of scientific knowledge without formally being recognized as scientists ("Users and Peers" 2). ${ }^{30}$ That to a certain degree maker culture (and 
its values) paved the way for citizen science is visible in the importance of the internet and the sharing practices promoted by makers. Citizen science has become "more diffused" through the use of Web tools for cooperation, collaboration and communication: From online discussions, to large scale efforts of data collection, procession and analysis as "volunteer scientists" for big institutions to independent and communitydriven $\mathrm{P}_{2} \mathrm{P}$ science, where design research, experimentation and analysis is done in the community (Delfanti, "Users and Peers" $1-2){ }^{31}$

DIY biology is often considered an offshoot of the open-science movement (Ledford 651) and amateur science tradition (Delfanti, Biohackers 2) as much as of the maker movement and hacking ethos, visible also in the alternative term "biohackers" (cf. C. Anderson 221-22). In 2005, a citizen science/biohacking group called "DIYbio" was founded in the United States, and their name has resonated so much that it has been taken over as an alternative name for the whole movement. It is thus noteworthy that while the biohacking movement has spread around the globe, the first and in fact most of the groups today are located in the US (Meyer, Domesticating 7). ${ }^{32}$ Borrowing principles and practices from all of the aforementioned ideological sources, the basic values and ideas of 'biohacking' are knowledge sharing, a community orientation, a pinch of rebellion and a passion for opening the doors of the 'ivory tower' in order to let 'lay' people participate in the wonders of science. DIY biology tries to "extend [hacker culture's] idea of openness to biology" and is often practiced as and in resistance to the "closed" biology of institutional science and the biotech industry (Stevens 351). Correspondingly, the goal of DIY biology is to move biology out of institutions and into the public (Delgado 66), to make "biology for the people" (Bennett et al. 1109).

Alessandro Delfanti specifically focuses on the connection between DIY biology and the hacker movement, claiming that DIYbio is an embodiment of hacking's fundamental values, such as openness, sharing, individualism and rebellion (Biohackers 125). Biology, here, becomes a strategic site on which these values can be played out and negotiated, in relation to big cooperations and government bodies, but also individuals, their bodies and surroundings. It is at once private and public. However, Delfanti also claims that despite the deep roots in hacker culture, the commonly used name "DIYbio" and its explicit reference to do-it-yourself culture "positions DIYbio within an old American movement of makers and inventors who work in their garages, and also gives it a rebel flavour." He thereby emphasizes the connection between the resurgence of the DIY movement in the guise of 'maker culture' - also as an 'American' movement - and biohacking, visible also in the use of similar communication tools such as Make Magazine or the website Instructables (Delfanti, Biohackers 118-19). Indeed, Make Magazine was one of the early publications that explicitly included 'making' with biology in their projects.

31 Zooniverse, for example, is one of these platforms on which volunteers can contribute to "people-powered" research in diverse fields such as arts, biology, climate, history, language, literature, medicine, nature, physics and social sciences. Without specialized training or equipment - a computer and internet connection suffice - volunteers assist 'professional' researchers and research projects using the 'wisdom of the many' to recognize patterns, classify and analyze information. Like in the hacker movement the Massachusetts Institute of Technology (MIT) had a foundational role: Like the first hacker groups in MIT labs, the first DIY biology groups were located in San Francisco and Cambridge, representing new and old elites. 
One of the first issues, in August 2006, was dedicated to "Back Yard Biology" (07, August 2006) - turning the homes and yards of ordinary people into spaces for experimentation. ${ }^{33}$

Space and place, in fact, are some of the more decisive geographical and material aspects of biohacking. ${ }^{34}$ While some groups meet informally or share spaces with hacker or maker spaces, the past ten years have also seen the creation of labs with secondhand or donated equipment and "hacked solutions," such as self-built, open-source instruments. Such labs are often modeled after hacker spaces, in that they are collectively run, community-oriented and shaped by similar political approaches, such as openness (cf. Delfanti, Biohackers 112-13). Following the idea that "biological information, knowledge, and techniques should be free, unlimited, and without ownership" (Stevens 351), these community labs give workshops on different biotechnologies, can facilitate informal mentorship and hands-on training (also for students and school classes) while simultaneously giving more experienced biohackers a cheap and well-equipped space to conduct their experiments in. ${ }^{35}$ The DIY biology movement, however, has also been known for more informal laboratories in garages and kitchens - the 'backyards' of 'ordinary' people.

The 'hacks' performed in these various settings range widely: Some of the projects are aimed at fun tinkering - for example creating fluorescent bacteria or yoghurt others at self-knowledge - such as extracting and analyzing one's own DNA. Some try to solve medical problems or to build alternatives to scientific equipment, accepted health care practices and technologies. Others are creative, such as biological art. While much of it is educational - public workshops and outreach activities, student education and discussion rounds - only small parts are cutting edge revolutionary or truly dangerous. ${ }^{36}$ Ana Delgado argues that DIY biology is focused on more routine making of (living) things: instead of producing "sophisticated biological objects" or the scientific innovations of institutional science, it engages with and produces more "mundane" (living) things, thus embodying "a different way of engaging with science and technology" (66). Everyday materials - what Stevens calls "whatever they can get their hands on" (351) - but also more specialized tools and materials are used for those experiments. In

33 Critic Sara Tocchetti looked at how "backyard biology" as a category was employed by Make magazine: She concludes that the magazine included both "hacking" in the form of "hacking your plants" (through e.g., hand pollination) and bio-engineering as a "hack" in their articles and proposed projects. In these, she claims, the backyard becomes a place of experimentation and analyzation, biology becomes a "material for personal experimentation" (Tocchetti n.p.).

34 In Domesticating and Democratizing Science, Morgan Meyer focuses his analysis of biohacking on the spatial-material configuration and enactment of the phenomenon.

35 Some labs in the US collaborate with local high schools to make their spaces accessible for student education. What needs to be acknowledged, however, is that despite the aim of openness and access most of these labs work with fee-based systems: Membership fees for regular access, course fees for their outreach activities, thus restricting their very ideals along socio-economic lines.

36 The specter of bioterrorism is evoked again and again, especially in popular media discussions and amongst institutional scientists. Synthetic biology does play a role, but not in a way that makes it truly dangerous - at least not today (cf. also Ch. 10). 
all of them, however, biology becomes a resource for experimentation, a material that can be tinkered with.

The potential ascribed to this trend, as in the maker movement more generally, is one of empowerment and democratization, giving people access to their own biological data as well as biological knowledge about the world around them, fostering engagement and education (Meyer, Domesticating 2). This educational focus, I will argue, can promote a scientific literacy that is needed for the public to not only participate fully in the (political) decision making around biotechnology and its applications and use, but also to make the most productive use of contemporary biomedical technologies, such as direct-to-consumer genetic testing, molecular tests or even tracked biological data: The other sphere of contemporary DIY that is the topic of the next pages.

\subsection{DIY Medicine? - Doing and Making in the Healthcare Sector}

The grassroots nature of DIY is also visible in how its key principles are carried over into different contexts, here, into medicine. In line with some of the basic cultural values discussed above, health care in the US is a highly individualistic enterprise, based on market rationales and a consumerist idea of the patient (Hall 43, 268-69). The rise of personal responsibility in a medical culture based on risks and susceptibilities, patient empowerment, shifting relations between medical professionals and patients, as well as the Web as communication and information tool have over the past decades contributed to fundamental changes in the health care economy. Patients are reconceptualized as 'consumers,' gathering information, browsing and weighing their options. In the name of empowerment, they are responsibilized to take an active role in their health care, to assert their choices in the medical encounter also against professionals. These developments are preconditions for current DIY trends in that they propagate self-responsibility, self-reliance and self-direction.

This turn towards individualism is foundational for numerous visions of future health care. As many critical theorists and practitioners imagine it today, the most promising developments in health care are consumer-oriented, individualized health solutions through technology: They promise to be more cost effective, targeted and based on prevention instead of therapy. "eHealth" is one of the avenues (and terms) praised for its potential to reduce costs while increasing quality, safety and access to medical prevention and treatment - reached in essence through the employment of information- and communication-technologies in medical settings (cf. Andelfinger 27). Part of this is the growing trend towards "personalized medicine:" Instead of a "onesize-fits-all" model, personalized medicine espouses a preventive, participatory model based on the individual and their own "data," which promises to be better equipped to deal with an aging population and increases in chronic diseases (Sharon 95). Examples include personalized, targeted therapies such as genetic or molecular diagnosis to evaluate individual responses to drugs as well as personal health technologies to gather data and take a more personalized view. These visions of future medicine are focused on the individual, involving them in the process of 'data' collection and decision making. One of the downsides is that here we are witnessing the making of a new private health- 
care sector, in which personalized approaches are not just restrict to those economically well-off but might also undermine successful community-oriented interventions. ${ }^{37}$

\section{Active Patients and Personalized Medicine}

Patient empowerment and personalized solutions are key and center of 'DIY medicine' and thus deserve closer inspection. During the $20^{\text {th }}$ century, medical encounters often left patients feel exposed, helpless, victimized and without control, especially during hospitalization due to the dominating power relations and authority given to doctors (Lupton, Medicine 96) - perceived as the sole authority over health and illness, the repository of truth about the body. Such feelings gave medical encounters a bitter aftertaste and contributed to a reversal of power roles, so that power today is increasingly spread evenly between the actors in medical encounters. For one, dissatisfaction with the conditions lead to a growing movement encouraging patient assertiveness that eroded the traditional power of doctors and turned patients into active, resourceful participants (Lupton, Medicine 113-15). They were expected and encouraged to do their own research and then take that newly gained knowledge into the medical encounter, challenging the doctors' authority. The internet, some argue, changed the "balance of power" in favor of the patient, allowing easy access to information, the possibility to meet and interact with doctors and other patients, and take an active part in diagnosis and treatment using information from health websites (Oudshoorn and Somers 205). With the wider availability of information on diseases and treatment options, the medical doctor is no longer the only source of information, so their authority is no longer given or (blindly) trusted. This new medium, on the other hand, can also become a source of confusion and anxiety: The wealth of different, in some cases contradictory information, questions about the reliability of sources and their sheer number can turn the gathering of information and their evaluation into an impossible task (cf. Ch. 11.3). Another part of the critique of medical authority and paternalistic relationships is the increased commodification of health care since the 1970s. This commodification has embedded patients into a discourse of empowerment that aims to create responsible, informed consumers, which tend to their own needs, for their own sake and that of society as a whole. More and more medical products are marketed directly to consumers to improve their own health, wellness or simply appearance, as the example of cosmetic surgery shows (Conrad, Medicalization 16-17).

The desire to be in power - to be informed and in charge of one's own situation - that is such a central part of DIY medicine today, thus, is also the result of a radical change in the US health care landscape during the last half of the $20^{\text {th }}$ century. Control, in this neo-liberal, individualistic marketplace of health, lies not with authorities but

37 One example is vaccination: On the one hand, those well-off might be able to better protect their children because they are able to pay for vaccinations out-of-pocket. On the other, the high value given to personalized approaches also gives rise to problematic aspects such as anti-immunization movements that risk undermining herd immunity as a successful example of community-oriented healthcare. 
with the self; only the self can control the self and pay for the tools needed to do so. Previously thought to be the result of meticulous medical training, the competence believed necessary for such an authority can today be facilitated by technology. Contemporary medical power, thus, does not play out in the form of domination and control but in monitoring and surveillance, discourses that make the intimacies of the patient visible, subjecting them to continuous analysis and record-taking (cf. Have 299). Active participation in health care choices has become a cultural imperative for the (neoliberal) subject, shifting the responsibility and blame for their own wellbeing to themselves and thereby encouraging ever more self-interventions and self-screening. ${ }^{38}$ Coupled with the pervasive discourse of risk and susceptibility, this trend makes it necessary for patients to collect even more information on themselves in order to make informed decisions and thus take control into their own hands.

For Till Hänisch the starting point for a broader implementation of personalized technologies is not an initiative on the side of health providers or legislation, but the more widespread adoption of practices such as the Quantified Self movement. He conceptualizes these as the start of an "extensive personalization of health care systems" [my translation], in which the patient takes an active, maybe even central role in health care provision - now oriented towards prevention ("staying healthy") and improvement of life quality instead of cure and extension (6-7). What Hänisch calls "eHealth," following the World Health Organization's definition, has as its goal the personalization of therapy on the basis of individual circumstances. This new individualism in health care, he argues, is also due to the increasing desire for attention to and consideration of individuality, already visible in the high appeal of alternative medicine. Maybe, Hänisch muses, "eHealth and Quantified Self will be the modern form of holistic medicine" [my translation] (7). This statement points to the "deep continuities" between new (DIY) technologies and practices and those that came before them (Greene 306). Complementary and Alternative Medicine (CAM), self-help discourses and self-medication can be seen as precursors for today's more sophisticated and invasive technologies.

\section{CAMing It}

Apart of - or rather part of - the growing focus on patient empowerment and consumerism, the past decades have also seen the rise and increasing incorporation of holistic, alternative approaches to health into 'orthodox' medicine. 'Complementary and Alternative Medicine' (CAM) espouses simple, non-technical and non-invasive treatment methods many of which are patient-chosen and paid for out-of-pocket. Many of these are age-old techniques that with the rise of biomedicine had become regarded as 'unscientific' but have in the past decades enjoyed a renewed interest among healthconscious groups. ${ }^{39}$ The National Center for Complementary and Integrative Health (NC-

38 This ideology of course tends to neglect those patients who either cannot (due to lower levels of education or literacy, for example) or will not fill out this role: This could be the case, for example, when patients have lower levels of education or literacy or when they perceive hierarchical distributions of power as a necessary part of medical treatments. 
$\mathrm{CIH}$ ), a US government body responsible for research on medical practices and products that are not commonly considered as part of conventional medicine, lists several different types of Complementary Health approaches on their website, among them natural products - such as herbs, vitamins, mineral, probiotics, and dietary supplements - and "mind and body practices" such as homeopathy, chiropractic and osteopathic manipulation, meditation, massage therapy, acupuncture, relaxation techniques, and movement therapies. Moreover, other approaches - taken over from other cultures - such as yoga and Ayurvedic medicine or traditional Chinese medicine are also included in their list.

All these approaches have in common that they conceptualize health and the body in a holistic manner. Such holistic health approaches do not just include medical concerns - a narrow, technical focus on symptoms and disease - but assume a broader focus that includes nutrition, psychological and spiritual well-being, relationships as well as environmental influences (Lowenberg and F. Davis 581). This holistic view of illness, however, follows the same ideology of active participation in the maintenance of health, maybe even more so (Lupton, Medicine 125). While consumers already became increasingly responsibilized for their own health choices during the commercialization of healthcare, this responsibility is heightened in holistic health: individuals are accountable for their own wellbeing (or absence of such) and compelled to prevention (cf. Lowenberg and F. Davis 588,593). Even for healthy individuals there is always room for improvement and optimization, an approach that forces them into constant surveillance of their own bodies. The patient is encouraged to closely monitor their physical and psychological status, actively change her lifestyle, and seek out information and experts in the name of pursuing health. ${ }^{40}$ While the symbols and rituals of both medical paradigms differ, they tend to remain mysterious for most patients and require not so much understanding as faith or belief in them (Lupton, Medicine 126-27). Thus, also in CAM, specialized knowledge is sold as a commodity, creating barriers of access for consumers who cannot afford to use such technologies (Lupton, Medicine 125-28).

Even though some CAM practices are practitioner-based and thus need the interference of an Other in the process, they are most often chosen by the patients themselves. The agency, at least on the surface, traditionally lies with them. ${ }^{41}$ Others can simply be taken on if a patient feels like this is the right 'treatment' or solution to their problem. The Web as information medium provides not just free tutorials on mind and body practices but also guidelines on self-treatment with dietary supplements as well as corresponding shops. Part of the medical consumer market, these practices are marketed

1990 and 1997" primarily because a higher proportion of the population sought alternative therapies, from $33.8 \%$ in 1990 to $42.1 \%$ in 1997.

40 While some point out that CAM challenges medicalization by bypassing the medical model and medical professionals and creates more informed and articulate consumers (cf. Conrad 11), other critics like Lupton argue that the quest for holistic wellbeing or total health, in contrast, adds to the medicalization of western culture (Medicine 127).

41 The question of agency becomes more problematic when we consider how the discourses in health-conscious parts of the population have turned some practices considered to be CAM into 'mandatory' activities for their members. Agency, here, becomes a pretense that is part of their ideology. What comes to mind are practices like Yoga and meditation, or dietary supplementation (think Omega-3) and probiotics. 
directly to the consumer as means to fulfill their ideal of health, fitness and wellbeing on their own accord. However, many of these practices are not 'quick' or easy solutions. Rather, the patient is responsibilized to stick to their treatment regimens, whether they involve regular practice or daily intake. Thus, work on the self - mentally, physically, emotionally - by the self is required to fulfill the ideal of holistic health behind most of them.

\section{Self-Help Discourses}

Work on the self is also an inherent part of self-help practices and discourse. From its beginning as a group and community effort of mutual support and the pooling of resources (Withorn 510-14), self-help discourse has morphed into an individualistic enterprise focused on self-transformation. This change is also visible in some of the DIY techniques under consideration in the later chapters, warranting a short historical view of self-help in American culture.

Micki McGee argues that until the 1970s, self-help was characterized as a cooperative, political effort by a community of peers (18-19). Still practiced today, this type of traditional self-help often takes the form of self-help groups. Self-help groups, online and in person, emphasize community, information exchange, collective (political) activism and mutual support and empowerment. The social and collective support provided by such groups was meant to help individuals with personal, family, or emotional difficulties and to achieve personal change or reach their goals (Withorn 510-14). Alcoholics Anonymous, established in 1935, with its Twelve-Steps program is emblematic for this type of self-help and served as a guiding principle also for other groups, primarily in the area of substance abuse. Following a similar structure, Weightwatchers can be seen as emblematic for self-help groups focusing on self-transformation. Collective activism, information sharing and social support are primary goals of patient and disease victim groups, such as for cancer survivors or chronic health conditions. As information- and support groups this type of self-help can be conceptualized as peer-to-peer support, fostering a sense of belonging and community in which people learn from each other's experiences. ${ }^{42}$ Like the maker movement or DIY biology, they are a collaborative effort based on open sharing of experiences, knowledge and information. In successful cases, self-help becomes transformative for the self, in that problems can be solved and solutions found together. This 'do-it-together' attitude, however, is not about making but about doing (something), about taking action to improve your own situation and to help others in a similar situation.

However, since the late $20^{\text {th }}$ century, wide parts of self-help culture has morphed from one focused on mutual aid and support into an isolated, individual 'undertaking,'

42 Even though the impulse for communal support is a healthy one and the emphasis on sharing and a like-minded community counters isolation and feelings of alienation, social components and structural difficulties are often disregarded in favor of a high focus on the individuals and their need to work on themselves (Withorn 517-518). Creating new forms of "biosociality" (Rabinow), these groups continue discourses of personal responsibility and expand the normalizing gaze to peers (Lupton, Medicine 128-29). 
focused not that much on collective, political action but on self-fulfillment. According to McGee, this focus on self-fulfillment has expanded the scope of self-help indefinitely because there is no end-point, no conclusion to such projects of self-making (18-19). The self, the body in this individualistic self-help becomes a personalized project. As McGee writes, "[i]magine a self and then invent that self [...] [p]icture a life, then create that life" has become the motto of American self-help - one that "has long infused American culture with a sense of endless possibility" (11). These endless possibilities are also highly visible in popular culture: A trip to the bookstore will reveal countless shelves filled with books, manuals and guidebooks on how best to go about your personal project of selfimprovement and self-empowerment.

Self-help books, in fact, are to a certain degree emblematic of the erosion of collectivity and the rise of individualization: Reading self-help literature is not a communityoriented but a solitary activity, not focused on exchange but on individual work on the self. In 1859 Samuel Smiles published Self-Help, a widely-sold book on personal development and character building based on values such as hard work, morality, thrift and perseverance, that turned out to be a precursor, and in fact eponymous for the whole genre. It was however not until the last half of the $20^{\text {th }}$ century that the US saw the explosion in self-help or self-improvement literature we see today. These self-help books typically span from psychological topics (work on the soul) to physical fitness (work on the body). What they share is, according to Sandra Dolby, a distinct combination of self-improvement content, an informal style, a problem-solution structure, and an educational function - turning them into an own genre of popular literature (37). This genre, argues Dolby, is commonly used as part of a self-education process in which people create a "self-directed" course of study for themselves that addresses their concrete situation and context (viii). The self-help genre has come to be a distinct element of American culture, as Dolby claims (37). For her, self-help books are a cultural resource that reflects and perpetuates foundational American values. Authors often draw on ideals and wisdoms deeply engrained in American culture and heritage and pass them on to the reader, for whom the books become a way to personally "experience" culture and incorporate some of its core values into their own worldview. As such, readers of selfhelp books are both embedded into a wider community and culture and solitary learners on a path to self-education (Dolby viii). ${ }^{43}$ Today, this type of self-help has reached past books into audio-visual and multi-media channels.

Most self-help books promise some sort of transformation, to become better, healthier, happier, more productive, successful and satisfied. Economic success, intellectual advancement, emotional or physical health, losing weight, increasing one's performance, self-confidence and positive thinking are typical topics of self-help discourses. ${ }^{44}$ They are marketed and sold as tools for self-optimization and improvement

43 Dolby argues that the "underlying assumption" of self-help books is "the prominence of the self" in American culture. The community is a concern, but the individual comes first also in many selfhelp books (21-24).

44 Medical advice books can be considered as part of this self-help genre. Gathering information about how to identify, treat and cope with health problems can be seen as self-help more narrowly. This is by no means only a recent trend. Medical advice books have since the $18^{\text {th }}$ century distributed information on illnesses and their prevention as well as desirable health-related be- 
in almost all aspects of life. This is reflected in the basic structure of all self-help books, the identification of a problem or a lack (in ourselves, our culture or the information about the world we possess) and the suggestion of a solution (Dolby 5). This structure, though superficially empowering the reader with new knowledge, always engulfs them in a discourse of deficiency, in that the appeal to self-improvement necessarily defines the reader as always insufficient in some regard (McGee 17-18). ${ }^{45}$ The focus, again, is on personal responsibility for your wellbeing and active participation with knowledge being key to success (Lupton, Quantified Self 50-51). As a contemporary technique of the self, self-help discourses become a means to display and perform a desired identity, to shape your conduct, self, and body through psychological and material work on the self. Like Gelber's view of DIY home improvements, self-help becomes a form of "productive leisure," work-like but now focused on psychological and bodily improvement. Similarly, for McGee self-help or self-improvement is a new form of immaterial labor that is expected of the individual. As such, the individual has become a "belabored self" burdened with constant evaluation, management and reinvention of the self to remain competitive (16).

This is a very much consumerist undertaking: In personal coaching, (online and offline) seminars, books, videos or tutorials 'experts' sell their knowledge to the uninitiated who want to 'transform' their lives for the better. A whole array of marketing techniques creates a complex of artificial desires and needs that self-help promises to fulfil. It is no wonder, McGee argues, that the popularity of self-help rose in economically precarious and uncertain times: Insecurity and unpredictability in the labor market and personal life call upon individuals to work on and invest in themselves, to improve themselves to remain competitive, attractive, valued (12). In self-help, actual lives are continuously assessed against some imagined future, a "pleasurable pornography of possibilities," a promise of power that far from actually bringing pleasure, according to McGee, is a recipe for dissatisfaction and erosion of collectivity in favor or narcissistic individuality (158-60). Contemporary self-help, following this line of thinking, is propped up by some of America's core values as well as a complex network of affective structures, most prominent among them anxiety and desire.

To summarize, I would argue that DIY Patients are nothing particularly new. But today the tools at their disposal are much more sophisticated, knowledge easier accessible and socio-political as well as economic factors in their favor. For a long time, paternalistic doctor-patient relationships resulted in feelings of helplessness and alienation on the side of the patient. Already older DIY techniques in medical contexts, their empowerment and self-starter attitude, can be read as a symptom of this strained distribution of power. Coupled with a commercialized responsibilization of the patient as

haviors. Around that time, self-medication through lifestyle changes and herbal remedies had been common (cf. Lupton, Medicine 83). While that has changed wildly with the professionalization of medicine, advice books and guides to self-medication - especially in the area of homeopathy, CAM and over-the-counter drugs - are still commonly available, even more so through the Internet. In this case, the patient-consumer becomes diagnostician and practitioner in one. We could speculate that this fact, and the sheer number of self-help books out there, might also lead to some form of 'self-help dependence' in which readers move from one self-help book to the next, never knowing what to do, which information they can trust and which they can ignore. 
consumer and prudent citizen committed to health and fitness as personal goals, they have normalized self-reliance, self-responsibility, self-direction and self-improvement also in biomedical contexts. Most of these common DIY techniques are more abstract forms of medical DIY, focused on psychological changes to health as well as collective action, means of participating in the medical encounter and decision-making processes yourself. Those that come closer to the material nature of contemporary making are included in the next part of the book, since those are the 'new' forms of medical making that are my primary concern. It is, however, highly interesting how many of those 'old' practices find their way into some of the 'body hacks' looked at in the next chapters.

\subsection{Coda: The American Maker}

"Off all things Americans are, we are makers. With our strengths and our minds and spirit, we gather, we form and we fashion. Makers and shapers and put-it-togetherers" (Banes and Hoag, 0:16)

Like the opening quote for this chapter, these words are setting the stage for a 1960s patriotic documentary, produced by the automobile industry under the name American Maker. ${ }^{46}$ Individualism, self-reliance, self-sufficiency, autonomy, have as fundamental American values paved the way towards this diagnosis. Do-it-yourself is omnipresent in US culture, not only in its crafty sense of home-made alternatives to commercial solutions but also ideologically, and possibly more pervasively, in the sense that you need to take care of, look after your self yourself. DIY is not a purely American phenomenon, but one that is much more prevalent in the US: especially the DIY activities under consideration here have their birthplace in American culture and contexts. ${ }^{47}$ Part of an old movement of makers, tinkerers, inventors and hackers, they are, essentially, old practices in a new guise, continuations of foundational American myths with tremendous formative effects, such as the Horatio Alger-style rags-to-riches stories or bootstrap ideologies such as the American Dream. In press accounts, maker culture and DIY biology are often represented as part of a long tradition of American innovation, that last raised its head in the tale of Silicon Valley as the worldwide hub of software innovation (Delfanti, Biohackers 117). DIY, thus, is integrated into a (proposedly) unique American myth of innovation, entrepreneurship and self-reliance: Making is not only fundamentally human, it is fundamentally American.

As many scholars point out, however, 'making' or 'doing' is not a new phenomenon, but rather today it has entered a new phase in which it is experienced as having a new

This documentary - a promotional video for 'made in the USA' - does not just perpetuate the ideology of American exceptionalism, it also perfectly reflects the problematic gendered divisions of 'making' in the 1950 s and 60s. Its ideological scaffolding should thus be seen critically, an emblem of its time and place.

47 The first DIY biology groups were founded in the US and medical DIY is more prevalent in the US, also due to its market-based health care economy and lack of comprehensible health insurance for all, cf. for example Keulartz and van den Belt. 
quality. It is a phenomenon that goes back decades, but that with a new coating is experienced as 'new' (cf. Gauntlett 61-63; Bal et al. 157-58). According to Gauntlett, part of this 'newness' is a different form of visibility, empowerment, inspiration, and connection especially through changes in media and communication technologies (61-63). The DIY techniques included in my discussion are a similar case: Neither biohacking or DIY biology nor the DIY patient are new phenomena. Delgado argues that DIYbio is not an original or isolated phenomenon, but "emerges as one of many DIY outbreaks that we see today within different sectors of the public," a proliferation of making, mending, hacking of different kinds (66). ${ }^{48}$ The new quality of these developments is the result of tremendous technical and biomedical advancements during the past decades. These have not just made them easier to do yourself, but have also raised the stakes both for individuals and the community.

Moreover, the DIY trend is the result of its socio-political and historical contexts: The political, social, cultural climate also in the US points into the direction of more participation and activism, in different forms and in certain circles. DIY citizenship, participation in decision making processes is part of a larger (political) shift - ranging from absurd and small-scale decisions, to large ones such as national budgets. More participation, thus, has come to be a cultural consensus. Many of the practices under discussion in my project, in this regard, are not necessarily isolated processes, but point to a larger yearning to be heard and acknowledged, to be part of the decision-making process and not subject of it (cf. also C. Lee). ${ }^{49}$ In this context, the contemporary "Doit-yourselfism" (Frauenfelder 217) also has a transformative potential for the material world as well as public life: fostering engagement and communities in individualist societies, bolstering confidence and competence in an ever changing world, providing possibilities to intervene in established systems of power. ${ }^{50}$ Also leisure activities can be political because they directly influence - generate and reproduce as Gelber phrases it - the structure of society. No matter whether they are mainstream pastimes or counter cultural ones, they are part of the "mutually compatible and reinforcing" public institutions that form the fabric of everyday life (Gelber 11). Ratto and Boler argue in a similar way that making can be understood as political because it invites critical reflection on

48 A similar stance is visible in Keulartz and van den Belt: "But the significance of its incipient paradigm of knowledge production is not limited to the DIY-Bio movement. DIY-Bio is not an isolated phenomenon but has emerged as one of many Do-It-Yourself initiatives that we see today within different technological domains (food consumption, repairing and re-using electronic waste etc.) in which citizens manifest themselves increasingly as makers, menders, and hackers." (Keulartz and van den Belt 16).

49 Caroline Lee in Do-It-Yourself Democracy discusses public engagement and citizen participation in various (political) domains and decision-making processes. Examples include the decision-making processes and efforts at public participation in the rebuilding activities in New Orleans after hurricane Katrina.

50 For a similar argument cf. Gauntlett, who provides a discussion of the creative, self-transformative and community-building potential of making (245), and Ratto and Boler on "critical making" and its potential to disrupt, intervene in and critically reflect on established systems of authority and power (1-3). 
systems of power and ideologies, modes of production, and processes of interaction (13). Likewise, Gauntlett says that the growing maker movement rejects the wide-spread "sit back and be told" ideology and its passivity in favor of a "making and doing" culture oriented towards "creativity, social connection and personal growth" (11-13). Doit-yourself, it is implied in all these scholarly accounts, is also a form of socio-political intervention.

DIY biology and medicine, in my view, are the logical expansion of more traditional 'DIY' projects: In their process orientation, the satisfaction they bring and appeal they create, their self-directed and highly material nature. In DIY we are constructing knowledge about our bodies, selves and life in general from very much material practices. DIY in its essence is learning by doing, a tinkering with processes, the modification of matter for utility, fun, education or exploration. It is this essence that the practices and techniques under discussion in this book take over from historical DIY practices. For Delfanti, biomedicine is one of the spheres in which openness and accessibility is the most valuable and "culturally entrenched" (Biohackers 12). The ideology of openness and transparency so foundational for DIY biology is visible also in the DIY patient. Breaking down the doors of medicine, gaining agency in medical decision making, giving and gaining access to medical knowledge and technology, open sharing of information - these were and still are some of the most important goals of proponents of consumermedicine and self-help cultures. New technologies and the Web 2.0 sharing ethos make it all the more applicable and relevant today.

We need to be careful, however, to acknowledge that this is still an emergent phenomenon "looking for stability," to quote Delfanti: It is still immature and hard to grasp (Biohackers 25). Its contours and consequences remain open and are subject to the pervasive uncertainty that rapid technological change brings with it. Still, seeing it as the result of recent convergences and age-old traditions and values allows us to discern some of the concerns, potentials and problematizations social and cultural discussions need to consider. One contributor to Make inspired Frauenfelder with "his perspective of the world as a hackable platform, something to be remade and remodeled" to his own liking (21). It is this idea of life as a "hackable platform" that is emblematic of the pervasiveness of DIY: Everything can become your new project, life is your oyster. You might only need a bit of creativity and perseverance. The question remains whether or not this ethos of 'do-it-yourself' will reach a cultural relevance that equals a downright revolution in how people innovate, collaborate, and produce knowledge and material goods; whether or not it will achieve a fundamental change in how people perceive of themselves and their material environment. 



\section{PART III: 'HACKING' LIFE ITSELF}





\section{5 'Hacking' Life Itself - In Pursuit of a Definition}

Type 'hacker' into the Google image search and the first hits show a shady figure in a black hoodie, faceless, anonymous, crouched over a computer, with streams of digits in the background. The figure of the hacker - a prominent one in our digital, data-based societies - carries with it a dark connotation: secretive, unofficial, invisible, avantgarde. Type in 'life' and you find images of sunrises and scenery, success, happiness, people and plants. Life is inherently positive. The term 'biohacking' often used for activities that 'hack' life itself, is a perfect amalgamation of these contradictions: the Greek bios, meaning life, becomes a prefix for the hack: 'biohacking,' it seems, is also 'lifehacking.' So how can this 'dark' activity be applied to something connotated so positively? As the previous discussions have shown, this culturally pervasive image of the hacker only carries with it the shady connotations of the (illegal) 'hack:' breaking rules and laws, posing a danger to safety and society, stealing (intellectual) property, entering digital systems uninvited and undetected with malicious intents. ${ }^{1}$ But hacking as an approach is much more diverse: As I have pointed out in the previous chapter, it ranges from these illicit activities, to the coding of free and accessible software, activism for more access to computers and technology or simply an ideology of openness, inclusion, innovation and transparency. In their essence, hacks, according to Delgado and Callen, are experiments that "show that problems can be solved and that things could be done otherwise." They are an "experimental mode of inquiry" in which not the success of making them work counts but the process of trying (189). Today, the object that is hacked does not have to be code: technology, bodies, materials, feelings, lifestyles can be subject to this type of experimental inquiry and transformation. Hacking has left the realm of the virtual, digits and code and instead entered the physical world. In fact, in DIY biology

1 Interestingly, this illegality might come into effect in biohacking in cases where life has been patented: substances such as adrenaline, insulin and vitamin B12 count among the historical examples of biological patents, whereas more recently DNA sequences have caused new debates about the eligibility of biological materials for patents. The most prominent example in genetics is Myriad Cenetics' patent claim for BRCA1, one of the 'breast cancer genes.' As it was common in the early 2000 s, Myriad Cenetics wanted to patent its prestigious finding. This decision received a lot of public backlash, leading to a U.S. Supreme Court decision in 2013 that ruled naturally occurring DNA sequences ineligible for patenting. 
and medicine, hacking is inherently material, focused on tangible technologies, human bodies and biological matter. And more than that, it becomes an activity in which the individual pulls all the strings.

This third part sets out to analyze the different forms that DIY practices take in the context of medicine and biology. Proceeding in the form of case studies, it will span a wide range of techniques and cultural representations, taking up the diversity inherent in this new field of experimentation, knowledge production and public participation. Its diversity, ambivalence and novelty have so far made it hard to find a definition for what 'DIY biology' is, which practices, people and discourses are included, which are excluded. In fact, I would argue that we do not even need such a definition as the (intentionally) unstructured boundaries, its constant shifting and the rapid advancements of the technologies used would obliterate any attempts at definitions before they reach print. $^{2}$ Still, for the sake of my discussion and the sanity of the reader, I will attempt to disentangle some of the practices. Consider the following: 'Body hacking' is often also called 'biohacking,' but 'biohacking' is also a commonly used term for 'DIY biology.' Are those practices one and the same? In truth, they could be considered different practices in the objects they choose to experiment with, but they are related in their core ideas. Many of the terms seem to be used interchangeably both by practitioners and in the public discourses around them, so I will try to somehow differentiate how they can be thought apart and delineate the (open) boundaries that I have set.

Let us start where (presumably) no solid research but all private information endeavors start. ${ }^{3}$ If you look "biohacking" up on Wikipedia, you will land on a disambiguation page, signaling that this title would be suitable for many different pages. The information you find includes the German streaming television series Biohackers as well as links to the following pages:

"- Body hacking, the application of the hacker ethic to improve one's own body

- Do-it-yourself biology, movement in which individuals and small organizations study biology

- Quantified self, measuring various biomarkers and behaviors to try to optimize health

- Performance Psychology, improving ones mental and behavioural capabilities to boost performance"

(Wikipedia, Biohacking, 16. April 2021)

Further articles link to Nootropics, Nutrigenomics, and self-experimentation in medicine. A snowball inquiry into the links reveals that body hacking is said to refer

2 In fact, it shares this complexity with attempts at a 'definition' of DIY: The multiplicity of contexts on which the term is used, as I have shown in Ch. 4, means that there is no clear-cut definition of what exactly DIY is.

3 I would argue that Wikipedia as an information source is much more revealing about what public opinion perceives DIY biology to be than any attempts at formal academic elucidation. As an open knowledge base - contributed to by its users - Wikipedia has become the new online encyclopedia used by many people to gather preliminary information. While it is true that Wikipedia articles are susceptible to false or misleading information or plagiarism, they nonetheless reflect what the 'crowd' thinks about the meaning and definition of certain terms. 
primarily to practices of grinding, the biopunk movement and do-it-yourself body enhancements (linking Neurohacking, brain hacking, as a further reference page). The Wikipedia page for "Do-it-yourself biology" lists bioinformatics, genetic engineering, medicine, implants, and art as potential research topics, with community, open source and advocacy being important aspects in this research. "Do-it-yourself biology (DIY biology, DIY bio) [...] as a growing biotechnological movement," the entry reads, can be done for profit or open science innovation, as a personal hobby or community learning effort, in which often people with training mentor those without (Wikipedia, "Do-ItYourself Biology"). All these pages, as Wikipedia and its contributors highlight through the disambiguation page for "biohacking," are somewhat related, almost impossible to differentiate - also linguistically, as many of these terms are used interchangeably. The Wikipedia entries demonstrate how the public conception of DIY biology is characterized by a confusing and complex diversity of its subjects, practices and "related but distinct" sub-movements (and names for these movements) (Wikipedia, "Do-It-Yourself Biology"). What they agree on, however, is the importance of a hacker ethic and hacker culture as well as the associated values.

In 2015, Nootka St. Film Company, a small creative studio based in Canada, released a short documentary on YouTube titled: SHIFT: Biohacking Documentary. ${ }^{4}$ Part of their SHIFT online documentary series, this 13-minute video shows the two directors, producers and presenters Steve Adams and Sean Horlor trying to elucidate what biohacking is by talking to some of its practitioners. What makes this documentary interesting is their inclusion of several different attempts at a concise definition, reflecting the different types of definitions present in the cultural imagination. At the very beginning of the documentary the directors emphasize improvement as the end goal of biohacking: "Biohacking is a growing global movement where anyone, not just scientists, can use technology to improve the human body." For them, the essence of biohacking is the use of technology to enhance our bodies (Adams and Horlor). Another definition provided in the documentary by futurist Nik Badminton focuses on the diversity of the community and its approaches as the main characteristic:

It's very much a self-starting sort of alternative community that believes that as humans we could be so much more. That could be through nutrition, exercise, and diet, and even more extreme forms by ingesting all sorts of drugs, smart drugs, augmenting the body using technology, implanting the body with things. Biohackers are a little less planned and research and a lot more: Let's experiment. Let's see where we can go. (Adams and Horlor, 02:12-02:35). an entry into body augmentation with technology; implants, nanobots, exoskeletons as technologies with medical and rehabilitative potential; as well as genetic tests as a basic form of biohacking - using the gathered data to change habits before they become health concerns. A voice over also acknowledges that not all biohacking is concerned with modifying the human body, but can also study non-human beings as well (Adams and Horlor). The documentary has had 51.184 viewers up until 16.04.2021. 
While it still maintains human improvement as the end goal this definition also underlines the creative, experimental approach. The third definition, given by Amal Graafstra, once more underscores the diversity of the movement: "Biohacking is a big umbrella term. It involves everything from hacking DNA and bacteria and people to doing what we're doing which is essentially not shying away from putting devices into the body to enhance it" (Adams and Horlor, 08:51-09:04). Already this short excursion demonstrates that there is a variety of definitions out there in the public discussion and imagination, many of which include human improvement, interaction with technology and an experimental ethos. ${ }^{5}$

In light of this variety, I have chosen to include all those practices in the area of medicine and biology that are carried out by individuals, ideally out of their own free volition, that follow the ethos of do-it-yourself. Like Graafstra proposes I use DIY biology and biohacking as umbrella terms, which also entails that I too use them interchangeably as denominators for an overarching phenomenon. For me, this includes DIYbio as a social movement and all the practices that entails (from technologies, molecular biology, to art), grinding (implantable technology), body hacking (improving the body) but also commercialized solutions (such as genetic tests, fitness tracking, and so forth). What do all these seemingly disparate techniques have in common? These practices, in my view, are united by their experimental and creative (out-of-the-box) approaches, solution-based and goal-oriented thinking, self-directed and self-imposed nature, and open and accessible character. I explicitly include commercial DIY solutions for the simple reason that they to a large degree follow the same logics of self-responsibility and choice but are more widely available to a broader audience. I consider them a different expression of the same phenomenon. In 2001, Robert Carlson had predicted that biotechnology would move from institutionalized labs to small companies and eventually domesticated spaces like kitchens and garages (Keulartz and van den Belt 2). The small companies that constitute some of the DTC market could thus also be considered a 'way station' between institutionalized science and complete DIY techniques. Moreover, Keulartz and van den Belt claim that grassroots entrepreneurship is a common outcome of DIY biology in the US: Hacker-inventors use the paradigms of DIY bio and the publics' willingness to participate in order to found start-ups (17). Some of the case studies in my discussion show examples of this type of economic activity around the DIY ethos, such as Graafstra's biohacking company Dangerous Things (Case Study VIII). On an even broader scale, commercialized solutions could be considered as an inherent and to a certain degree required part of DIY approaches in health care. In countries like the US, with a focus on prevention and active inclusion of patients into their healthcare, Andelfinger and Hänisch argue, commercial DIY forms, such as webpages, tracking, DTC testing, apps, and gadgets, become increasingly important for health care provision ("Einleitung" 2). In a consumption-oriented society, the consumerist approach of DTC and commercial DIY practices might be a more appealing and accessible way to support those paradigms and partake in their perpetuation.

5 Academic considerations often shy away from a concise definition as well. Instead, the diversity of approaches, ideologies, spaces and practices is demonstrated, while carving out some of the common characteristics. An overview is provided in Chapter 4.2. 
What most of the DIY practices share is that they do not only have cultural, social or political effects but also very much material ones: Some modulate biological materials, others inscribe themselves on bodies and leave their traces, and still others aim at a more profound knowledge of biology (one's own and more generally) in order to use it for individual goals and projects -but they all to some degree affect biological matter. For example, Delgado argues that practices of DIY medicine turn "the body into a test field for medical innovation:" "people are actively using their bodies for producing new diagnosis and for testing treatments," not just when established practices provide no hope, as Delgado claims, but also when they are perceived as insufficiently addressing individual needs (71). The body becomes a test field for experimentation with all the material effects that can entail. Talking to different DIYbio members, Delfanti found that for some the political commitment of DIY also encompasses a more "proactive" attitude to an individual's own biomedical information, facilitated by access and transparency in biological practices (Biohackers 123).

Information about the body is perceived as encoded in its materiality; DIY practices can uncover this information and make it actionable for the self. The human body and its biology become a new type of imagined and material 'frontier,' a new territory full of possibilities that waits to be explored and conquered using the latest technologies. "Jamming stuff into your body, merging machines with your nerves and brain, it's brand new,' said [biohacker] Warwick. 'It's like this last, unexplored continent staring us in the face" (Popper). The body, in biohacking, becomes a charted, but unconquered territory ready for exploration, cultivation, and indeed exploitation. The increased attention towards the material reality of one's body creates a heightened self-awareness of the body and helps modulate the self accordingly. Bodily plasticity is a precondition for this process. The DIY techniques assume the body to be open to change on molecular and molar levels, be it through supplementation, extraction or integration: Molecular pathways are optimized, bodily fluids extracted and analyzed, technologies integrated into the body. All those either open up the body, quite literally, or leave their marks and traces. The entanglement of biomedical knowledge, technology and everyday life alters "how the body is constructed, seen, and talked about," mostly as a sphere that individuals have to shape and improve (Burri and Dumit, "Introduction" 5). Intimate knowledge is often coded as leading to some sort of self-improvement.

The social, cultural, political effects of biohacking are more abstract, concerned with how the self is conducted in society, how techniques are culturally perceived and sanctioned, their future applications and broader implications. I will come to those in the next part of the book, when I take a step back from the case studies to reflect on common themes and discourses, political claims and social consequences. For now, suffice it to mention that some of the core concerns will be questions of participation and literacy, changing roles of power, competence and expertise, feelings of uncertainty and insecurity as well as the repercussions of individualism for society at large. Even smallscale adoptions, like those discussed in my case studies, can further a process of normalization and routinization and thus a transferal of concepts to larger projects. For example, if vitamin supplements or fitness trackers are normalized, interventions using smart drugs or implantable monitors might be considered as the logical, necessary and sanctioned next step. DIY techniques create social change precisely through such 
decisions about their use - about how to use them, when to use them, who can use them or who has to use them (cf. Brodwin 3). These decisions are embedded in the personal and political stakes that individuals and societies have in them.

\section{Practices and Representations}

How do I approach my case studies in this chapter to elucidate both the concrete material effects of the practices as well as their social, cultural and political implications? In this part, I access those material practices primarily, but not exclusively, through representations, meanings and discourses. Probably more so than the practices themselves, cultural representations shape public discussions and can shine a light on how we talk about these practices, which hopes and fears are embedded into them, how they shape lives and bodies, and how they are publicly evaluated. ${ }^{6}$ My goal is to describe the relationship of individual 'texts,' broadly defined, to the cultural and biological imaginaries of the audience and producers of the texts and to elicit the values, discourses and politics inscribed into the texts - to show the 'cultural work' these texts do. The representations under consideration also invite reflections on assumptions and shared American values: In this case not necessarily (but also) assumptions about race/ethnicity, gender or class - the traditional three - but about the human body, its biology and our material surroundings. The representations range from accounts of DIY biology in newspapers and online magazines such as Wired, Mashable, or the New York Times; websites and product descriptions; popular science accounts and self-help books; to videos, films and documentaries. As this list makes clear, I am working with a broad understanding of texts and a wide variety of media. ${ }^{7}$ This variety is chosen consciously to demonstrate the range of possible texts individuals might encounter in public discussions. Let me provide a brief explanation for how these different types of sources are well suited to address some of the core issues of the DIY trend.

For one, most of them could be considered as classical 'pop-cultural' formats. Popular culture, in my opinion, is much better suited to capture on-going and timely cultural concerns than more avantgarde formats. They do not only reflect but also shape and feed dominant assumptions, stereotypes and imaginations. Victoria Pitts-Taylor argues that even if pop-cultural formats might be simplistic, exaggerated or misleading, they are still socially significant because "much of what people know about biomedical science comes from the press and from the experts in the self-help market" trying to reach their (lay) audience through a variety of (popular) media ("Plastic" 641). Audiences make sense of medicine and science also through the practices and products of culture - among which popular media are some of the fastest moving, most-consumed

6 Chapter 2.1 looks at the role of culture, and especially popular culture, for the sedimentation and translation of scientific knowledge, its relation to biology and role in the production of meaning from our shared biological materiality.

7 Krupar and Ehlers' methodology of biocultural studies suggests intertextual readings "between various kinds of cultural products, knowledges, policies and practices" to examine the separation between "discourse, practice and materiality" (7). 
channels. Popular media are one of the main avenues through which biomedical innovations and practices are communicated to lay audiences, many through some sort of science journalism or popular accounts of science. Popular accounts of science do not only perpetuate scientism as the dominant, culturally shared world-view but also show how fashionable science has become today (Erickson 174-75, 187-89). However, popular accounts of science are not just popular because they feed the public's desire to be informed but also because they are entertaining (Erickson 187-89). ${ }^{8}$ They have more leeway when presenting scientific facts than more esoteric forms of publication and popularization, such as scientific journals. Media accounts of DIY techniques and nonfiction science books are two ways in which science journalism enters my discussion, most prominently in Chapter 7 in my discussion of the DIY EpiPencil and its media representation (Case Study IV Make Your Own Medicine), the analysis of Wohlsen's portrayal of Kay Aull's famous genetic test hack in Chapter 8 (Case Study VI Make It Personal) and Chapter 10's discussion of Wohlsen's take on technological hacking (Case Study IX Cheap and Functional). A special sub-section of non-fiction (science) writing, self-help books, are the main subject of my analysis of biohacking 'manifestos' in Chapter 6 (Case Study I Be Smarter, Stronger, Happier). All these formats are especially relevant as forms of translation but also because from them we can infer common associations with DIY biology and its presumed relation to biotechnology more generally.

Because they fit so well into the contemporary audio-visual culture, documentaries are another popular and comprehensible medium that is used for the sedimentation of scientific 'facts' into the public sphere. Deborah Steinberg describes the documentary as a "genre of visual culture whose particular capital is 'truth'." As such it pretends to offer "unmediated facts" (132). Documentaries seem to be particularly appealing to contemporary audiences in the knowledge society, they are a quick and easy way of selfdetermined learning. My case studies make use of documentaries mainly as niche productions, such as the above quoted YouTube documentary about biohacking, but also in the form of popular shows, such as Netflix's Take Your Pills (2018), the object of interest in Case Study III Take Your Pills, Become Limitless in Chapter 7.

A more affective, desire- and need-based popular medium that I include in my discussion are advertisements and other forms of public communication, including websites, ads, and commercial videos. These are prominent sources in my discussion of Dave Asprey's Bulletproof brand and the new experts of the soma in Chapter 6 (Case Study II Biohacking on the Web), genetic testing companies' web presences in Chapter 8 (Case Study V "Welcome to You"), Chapter 9's discussion of technological integration through tracking and grinding (Case Study VII Quantifiably Me and Case Study VIII Implants, Grinders and Technological Immersion), as well as my consideration of tinkered medical tools in Chapter 10 (Case Study X Practical Solutions). Nelkin and Lindee argue that advertising images are significant indicators of public perception: They are made to sell ideas and products, are broadly understood and widely appreciated, and resonate

This type of "edutainment" makes the popularization through science journalists and writers a key component in the processes of translation and mediation of scientific knowledge, cf. Rose, Politics of Life 29-30. 
with consumer experience and popular belief (13). ${ }^{9}$ Fittingly, I use them to also elucidate some of the promises and affective atmospheres that are created around products and practices.

The practices and representations in my case studies, however, should not be considered a comprehensive list or extensive record. They are selective examples that I use as illustrations and sources for textual and discourse analysis. Many of them were found using Google searches with specific key terms or searches on web platforms like Amazon.com. ${ }^{10}$ Some I stumbled upon during my research, others are more widely known. This web-based approach was chosen to reflect the primary means through which an interested public finds these texts and practices - online, either on purpose or accidentally. It also aligns with the web-based focus of DIY biology and the maker movement more generally. I hope that the variety of sources presents a realistic view of what types of information, discourses and images an individual will encounter during their search for information or opportunities for participation. Each chapter first provides context and a brief introduction to the practices before looking closer at their representations. This way I hope to work out the specifics while not losing sight of common concerns. The order of chapters and case studies is not one of importance but rather tries to group some of these practices into separate but related clusters, which themselves are not always clear cut, but rather overlap and intersect.

Medical advertising is no exception: The USA are one of the few countries that allow regulated direct-to-consumer advertising for drugs. These do not only prompt patient-consumers to go 'shopping' for treatments, but they can also play a role in the conception of diseases and accepted experiences of illness. My MA thesis on the representation of Multiple Sclerosis, for example, has looked at the representation of people with MS in medical advertisements for MS drugs and found that most of them use a desire for (and promise of) fitness and activity, making the neurological disease all but invisible. As such, they might shape how affected individuals experience and evaluate their own embodiment, cf. Crewe-Salfeld.

10 Among those key terms are more generalized ones, such as biohacking, DIY biology, or DIY medicine, and more specific ones like grinding, genetic testing, or names of companies. 


\section{6 \#iambulletproof - A Layperson's Guide to Self-Enhancement}

DIY patients have become a contemporary figure not just in the medical landscape but also in American culture more generally: They are the personification of individualized solutions and active participation in their health and healthcare. This chapter looks at new guides to self-enhancement that build upon the (literary) tradition of selfhelp books as well as discourses of individual responsibility for one's own wellbeing. It describes how traditional self-help or self-improvement tools today are reframed as 'hacks' that enable you to actively optimize yourself, thereby combining traditional tools with newer, biomedical, experimental ones. As I have elaborated on in Chapter 4, the traditional form of self-help - a collaborative effort based on open sharing of experiences, knowledge and information - has today been mostly supplanted by individualized and commercialized forms of self-help. These are aimed at (neoliberal) goal reaching and productivity, producing what McGee calls a "belabored self," constantly improving themselves in order to remain competitive in the market place and reach some sense of security (17). The guides under consideration here are part of this individualized, commercialized effort to promote self-directed modulations of the human body.

McGee and Dolby give different reasons for why self-help is (still) so popular today, with the former arguing that self-improvement has become an economic necessity and the latter claiming that self-help literature has become a part of personal education projects. Both approaches are visible here: For one, the practices under discussion in this chapter have a clear link towards optimizing the productivity of the individual, to give them an 'edge' over the competition - an ideal born out of the vulnerabilities experienced in day-to-day life after the last economic crisis. However, they are also part of larger 'education' efforts with the aim of making biotech developments more accessible, understandable, and actionable for the masses. As I will demonstrate, the authors and companies behind the case studies see themselves as translators of knowledge. This type of biological DIY and self-help can thus also be part of Dolby's personal, self-directed education processes that enable the reader to incorporate new knowledge, new ideas and values into their subjective worldview. 
This is all the more interesting, as Dolby also argues, because such self-help books to a certain degree transport some of the values and basic propositions of a culture (xi). The writers, she argues, extract and re-insert an existing "American" worldview into their books, thus effectively "recycling" established values and ideas, giving them back to their readers, who then use them to affirm existing believes or insert new ones into their personal philosophies (1-2). The texts, therefore, can be seen as reflections of cultural concerns, contemporary material practices, hopes and values. Extending Dolby's argument, I argue that the self-help tools under discussion here perpetuate a view of biology and the human body that is already prevalent in American cultural ideas: a widespread belief in physical malleability, scientific solutions and individual transformation. Basically, the examples in this chapter are representatives of a new scientific and molecularized form of self-help that is spread not primarily via traditional media but through the channels of the Web (2.0).

As the examples in this chapter show, the five pathways in which "mutations" occurred that Nikolas Rose identified in Politics of Life Itself more than ten years ago molecularization, optimization, subjectification, somatic expertise, and economies of vitality - are still very much present today. The physiology of the body is framed in molecular terms; interventions happen not necessarily in the name of health but to achieve an optimal state of being; humans are responsibilized, subjected to new somatic duties and expectations of health, fitness and productivity; new experts of the body advice and 'coach' individuals on their 'journeys,' leading to new "economies of vitality," new forms of extracting biovalue from the inherent human desire for (physical) perfection. ${ }^{1}$ While some of those differ to some degree from Rose's original idea, their continued relevance serves to show that these tendencies are anything but new. Instead, they are taking new form, evolving along with the biotechnologies that make them possible. It is especially the latter two that have experienced new 'mutations' in the cases under consideration in this chapter.

The individuals behind those guides to self-modification consider themselves to be 'biohackers' or 'bodyhackers' in the sense that they are 'hacking' their own biology, their bodies, on a molecular level. DIY biology, thus, is here focused very much on the individual human body, biohacking understood as forms of self-directed physical maximization and modification. A lot of this work consists of charting its status quo through self-observation, experience and biomedical testing; finding 'individualized' solutions through self-experimentation; tracking results and thus enhancing your well-being and performance. ${ }^{2}$ The do-it-yourself approach is evident to varying degrees in the two

Coaching is one of the outgrowths of the new role of (somatic) experts. During the past years coaching as a profession achieved a new eminence: life coaches, career coaches, health and fitness coaches - people seem to be looking for new forms of guidance in more and more areas of life. Part of this rise might be that more information makes it harder to discern what is right for an individual, scientifically proven or even just logically sound. In a world of uncertainty, people are looking for a new authority that validates their choices. While I do not want to disregard coaching per se, I do want to highlight that that is has become more and more prominent during the past years.

2 I am putting individualized in quotation marks here because the personalization of many of the suggested solutions in the guides remains questionable. 
case studies. However, in both DIY is broadly understood as self-directed interventions: Even when biomedical 'professionals' are needed to perform the intervention, it is the self that is expected to make the decision. Moreover, here we find an amalgamation of practices that are also part of some of the following chapters, such as supplementation, testing, or tracking, combined with a playful, experimental mind-set.

For this chapter I have chosen to examine two case studies more closely: Guides to self-help in print and on the Web, more precisely, two guides self-published by their authors and distributed widely via Amazon and similar services - James Lee's The Biohacking Manifesto (2015) and Ari Meisel's Intro to Biohacking (2014) - and the body hacking brand Bulletproof founded by famous biohacker Dave Asprey. These guides offer knowledge and actionable recommendations, while emphasizing the need for personalized solutions - brought to you through their coaching. ${ }^{3}$ An important theme in both case studies is science, or the belief in scientific solutions, as well as access to information. Lee, Meisel and Asprey continuously stress that their recommendations are based on scientific findings, thereby transferring the perceived authority of science onto their own arguments. However, the scientific basis they claim is not undisputed: For example, critics, also in the media, have argued that Asprey often cites studies with only a small research base, studies solely working on animal models, or that he ignores contradictory evidence and takes study results out of context (Belluz). It is important to note that my aim is not to proof or disproof their scientific basis, nor am I even particularly interested in that. Rather, I am looking at the discourses that are created and the assumptions they perpetuate and those revolve around scientism and knowledge, responsibility and active patienthood, plasticity and optimization, and the promise of a better, longer, healthier life.

\section{Case Study I: "Be Smarter, Stronger, Happier" - Biohacking Manifestos in Print}

Ari Meisel's Intro to Biohacking: Be Smarter, Stronger, and Happier (2014) and James Lee's The Biohacking Manifesto: The Scientific Blueprint for a Long, Healthy and Happy Life Using Cutting Edge Anti-Aging and Neuroscience based Hacks (2015) are two examples of guide- or advicebooks now readily available for the consumer. Both were self-published by the authors and are available on platforms such as Amazon.com, where they rank among the first few suggestions when searching for "biohacking." 4 The books, as I will argue, are clear marketing tools for the services offered by the authors: individual coaching and consultations that provide more personalized solutions to health problems or fitness goals.

Both Lee and Meisel work as coaches/consultants and advertise their services in their guides, Bulletproof has its own "Human Potential Coach" training, that certifies "Bulletproof Coaches."

4 Other recent examples include: C. F. Smith et al.'s Limitless Biohacking (2017), whose subtitle "Gain an Unfair Advantage With Science-Backed Supplements Big Pharma Doesn't Want You to Know About..." points to similar discourses as those under discussion in this chapter. The main concern of the book are supplements that give you an edge, help you stay "in the zone" for a longer period of time, using "biohacking" as a systems-oriented approach to hack the biological machine that is the human body (cf. Foreword). "Health and fitness leader" Ben Creenfield's Boundless: Upgrade Your Brain, Optimize Your Body \& Defy Aging (2020) promises a blueprint to bring optimization from the sphere of lore and science into everybody's reach. 
They are meant to introduce the reader to what is possible and create a desire for personal transformation. Meisel's Intro to Biohacking is meant as an "introductory course" or seminar to biohacking, inspired by his own personal history of Crohn's Disease and his journey of improving his own medical condition (6-7). ${ }^{5}$ His narrative and promise, as established in the first few pages, is one of "curing the incurable:" If he managed to cure his chronic health condition through health hacks, surely the reader will succeed in their own journey as well. The focus of his book is on individual goal achievement and the idea of "unlocking" your personal potential (Meisel 13). Meisel seems to be focused on less specialized (scientific) approaches and more on commonly available and used resources, such as apps and websites or forms of self-tracking. Lee is an avid self-publisher and author of various books on different health and biohacking topics. His Biohacking Manifesto, thus, in essence is comprised of three different books he had already published, now updated and combined. In the introduction he describes his book as a manual, a "comprehensive guidebook" (Lee n.p.), that however, due to its structure lacks an overarching theme or narrative focus. Instead, the three jumbledtogether chapters look at anti-aging, the modulation of neurotransmission and brain hacks and nootropics, often repeating a lot of the same information.

What warrants closer inspection here is his title: The Biohacking Manifesto. It is this word choice that has puzzled me the most after reading through his very comprehensive manual. There are no real statements of political, social or even biological aims, purposes or policies. Rather, it is a collection of (scientific) information about biochemical pathways and systems laid out in a comprehensible manner and combined with suggestions as to how those pathways and systems can be modulated and optimized (Lee n.p.). Why then is he calling it a manifesto? It does not seem to be a manifesto in the traditional, publicly used sense, a political, policy-based one like Marx's Communist Manifesto or Meredith Patterson's Biopunk Manifesto. He also does not seem to intend it to be a manifesto people should live by. Even though he does include short tips and principles to observe, those are essentially old, common knowledge such as exercise, a healthy diet, socialization and meditation. The more advanced hacks he introduces, on the contrary, necessarily need to be individualized and experimented with (Lee, n.p.). They cannot be considered as generalized guidelines. Though slightly misleading, Lee's use of the word "manifesto" seems to be closer to the original Latin meaning of the verb manifestare: A means of revealing information, of making it visible, of making it public.

Can these types of books be considered self-help books in the classical sense? According to Dolby, self-help books are separated from other genres of popular literature through their content and function: Namely, that their content deals with some form of self-improvement, their style is informal and rhetorical, they follow a general problem/solution structure, and they have an educational function (37). For Lee and Meisel most of that applies: Their content, explicitly, deals with biological optimization and improvement; their styles are rather informal, colloquial, on par with the presumed reader;

5 One of the main selling points for his ideas, as he frames it, is his success of hacking his own body. Interestingly enough, the starting point for his experimentations with biohacking was a medical cause, a disease he wanted to find a personalized cure for-emphasizing the blurry lines between hacking for cure and hacking for enhancement. 
they detail a (biological) problem and offer a solution - not just in the grander scheme of offering a solution to human fragility but also more narrowly by outlining specific biological problems which their tools help overcome - and their focus is educational, as I will elaborate on below. Both can therefore be seen as a continuation of the self-help tradition with biological, molecular, and neurochemical tools. Fittingly, do-it-yourself in their guide books often takes the form of self-education and self-improvement; the self if portrayed as responsible and accountable. ${ }^{6}$ DIY becomes the favored route to success. Especially for Lee, this route includes self-experimentation as the best way to find solutions, or tools, that work for oneself. ${ }^{7}$

These tools, in both guides, are referred to as "hacks." Lee and Meisel position themselves in the tradition of biohacking, in that they hack their own biology. For Meisel, a "hack" is an "improvised solution" that is unconventional, surprising, "out of the ordinary," anything that "works for you." "With biohacking, we're essentially hacking our own bodies, creating unique...solutions for common health problems...." (5-6). The element of novelty and experimentation, that he puts so much emphasis on, is also visible in his construction of hacking as a "journey" (63), a process, a series of experiments. This process- and project-orientation perfectly aligns with broader ideas of DIY. More specifically, his suggestions of hacks include a mixture of basic techniques and more advanced ones such as: exercising regularly (practicing "the manmaker," interval training and yoga); getting enough sleep and improving sleep (through perfect timing, reduced exposure to blue-spectrum light, detoxification or lucid dreaming); eating selfcooked meals and super-foods such as healthy fats, coffee, avocados, wheatgrass powder, chia seeds, or spirulina or practicing intermittent fasting; supplementing krill oil, probiotics and Vitamin D (the universal three); improving productivity through optimization, automatization, and outsourcing; tracking the self in almost all aspects of

Meisel, for example, starts out his discussion with an element of intense personalization: "I am here to tell you that biohacking can create positive, dramatic, and sustainable change in your life. It starts with a first step. Are you ready to make yours?" (Meisel 8, my italics) This direct addressing of the reader emphasizes their accountability for their own life and the changes they want to make: They are the ones responsible for implementing them. His book lays open the tools they can use to reach their goals. These tools are chosen in a way that the individual biohacker-novice can use them themselves in a DIY attempt to modify their physical health and wellbeing.

7 A biohacker himself, Lee has done his share of self-experimentation with drugs and behavioral changes. He relates some of his experiences with " $n=1$ " experimentation to the reader, and even though he cautions about their generalizability, self-experiments for him are a valid and valuable route. 
life; ${ }^{8}$ and leaving time for regeneration and rest through Contrast Hydrotherapy (hot and cold showers), wearing compression tights, or earthing. ${ }^{9}$

For Lee, biohacking is intrinsically connected to the human body as well. In his introduction he writes that biohacking is "just a fancy word for using science-based tools and shortcuts for optimizing your own biological potential" (n.p.). This optimization or maximization reaches from longevity and life extension, to memory and cognitive function, neurochemistry and psychological well-being, physical performance and physiological health (n.p.). Lee puts his focus on biochemical pathways and their modulation through drugs, (natural) supplements and behavioral changes, for example increasing serotonin, dopamine, noradrenaline, and endorphins to optimize the brain (the easiest "fix," he writes, is exercise). Still, also Lee includes many of the practices that Meisel mentions, such as yoga, supplements and drugs, diet and nutrition, sleep and meditation, social connection and quantification into his considerations. For example, for both of them physical exercise is a very "powerful" biohack to modulate your body not just physically but also chemically, according to Lee. Many of the hacks thus are not necessarily new techniques. Rather, they could be considered generalized advice, traditional wisdom or part of older practices of CAM. ${ }^{10}$ The crux here is the linguistic reframing as a 'hack' meant to create a new cultural appeal. 'Hacking' implies novelty, experimentation, fun but also carries with it a notion of illegality, of individuals working against the system. These ideas upgrade old techniques for a new audience, make them seem technical and scientific - fitting for a society that favors technological solutions - and in some cases try to override stereotypical assumptions. ${ }^{11}$ As 'hacks' they are integrated into the prevalent culture of active participation, making, mending and doing. But they also buy into the same old narratives of progress and biomedical advances, thus building upon age-old values and principles.

What stands out is that Meisel seems to focus on data tracking and the quantified self. While this form of DIY biology is subject of the one of the next chapters, it is noteworthy that Meisel sees them as invaluable parts of a biohacking routine. He includes a whole chapter on tracking and the quantified self, in which he introduces techniques and tools to track aspects of daily life to optimize them, from productivity (Pomodoro technique) to health (insidetracker.com), ending in a long list of suggestions for apps and services (51-55). Data for him are a "pathway to self-discovery," which will show you potentials for improvement or unexplored health effects (63).

9 Through "earthing," according to Meisel, people aim at "reestablishing the lost connection to nature" through either walking barefoot or products like earthing bed sheets or mats (59).

10 As some of their biohacking suggestions already show, both Meisel and Lee pursue holistic approaches to wellbeing by considering mind, body, and spirit in their guides - another similarity with older CAM practices. For example, both argue for social connection and interaction as a means to heighten mental and thus physical wellbeing. In addition, Lee tries to bridge biomedical and natural approaches through focusing both on pharmaceutical drugs and natural supplements.

11 A good example is Meisel's introduction of yoga as a biohack. "Many people," he claims, "still balk at the idea of joining a yoga class. They may be embarrassed, uncomfortable, consider yoga too feminine or too new-age" (12). To override the spiritual and gendered associations with yoga in the Western world, he suggests downloading the Yoga Studio app on your phone so that you can perform your yoga routines in the comfort of your own home. It is time, says Meisel, to challenge the collective opinion of yoga. Yoga is only one of the examples of reframing in Meisel, who generally employs this technique more often than Lee. 
The focus of their considerations, as we have seen, is the human body. All of their hacks somehow try to modulate and affect it. Despite their evocation of holistic ideas of wellbeing, their accounts perpetuate a classical metaphor of the body: that of a machine. Meisel writes: "It's helpful sometimes to think of the human body as the most complex and sophisticated machine on the planet. That's really all the body is: a beautifully functioning, harmonious machine of interworking parts." (Meisel 27, my italics) He continues this line of thinking by comparing food to fuel for that machine. As such, the human is responsible for maintaining and caring for that machine. Fitting to his more generalized focus, this machine metaphor remains rather abstract in his guide. Lee's manifesto has a keener focus on details, and thus it is no surprise that the broad-level machine metaphor is here supplemented with a very much reductionist, molecularized thinking: Lee aims at modifying neurotransmitters, precursor substances, free radicals, the smallest units of the body. Even when he suggests holistic approaches such as socialization, exercise, or behavioral therapy, the explanations for how they work to promote longevity, enhance moods or upgrade the brain are on a molecular level. The body becomes a system with complex pathways and causations, whose modification is strenuous but possible (Lee). This minute view of the bodily system is key to his approach to biohacking. It reflects his - and the biohacker's - commonplace understanding of the human body. Taken together, their accounts represent a mechanistic-systematic view of the body so prevalent in contemporary cultural discourses. Here, Lee and Meisel perpetuate a view of biology and the human body that is already part of American cultural ideas and assumptions.

Moreover, Lee's molecularized solutions all imply some form of modulation, a plasticity of the biochemical pathways under questions. As a consequence, I argue that a belief in biological plasticity, especially brain plasticity, is at the core of both their considerations. In his discussion of nootropics Lee writes:

If we cast our minds back, we may remember that up until recently, scientists believed that the brain was fixed at birth and could not be altered in structure. However all that changed with the discovery of neurogenesis (the birth of new brain cells) and neural (or neuro) plasticity. Now we know that you can change your brain by repetitive behaviours ... and by selective modulation of particular brain systems,... (n.p., my italics)

Much more simplified Meisel uses the metaphor of a muscle and a workout to explain the general idea of plasticity. "Working out" the brain like a muscle through brain training games can in his words increase cognitive function and performance (15-16). This type of neuroplasticity is made most obvious in discussions of the brain and brain modulation, but it can be extended also to other processes bodyhackers want to modify. What is necessarily implied is that these processes are open to change, can be modified by specific behaviors in order to be improved. The plasticity of the human body is one of the central premises on which their ideal of biohacking rests.

This assumption of plasticity is therefore also the basis for the very idea of selfimprovement in their self-help books. Optimization is a core concern for both Meisel and Lee. Meisel introduces the aim of optimization already in the subtitle "How to be Smarter, Stronger, and Happier." Like the moderator of a quiz show or theatrical performance, Meisel introduces himself to the reader: "Welcome to the fascinating, ever changing, and always adapting world of biohacking. My name is Ari Meisel, and I've 
created this study to help you harness the cooperative power of mind, body, and spirit in order to generate a more fulfilling, healthier, and more productive life." (5, my italics) From the beginning, the reader is promised that this book will help them to optimize their life. Afterwards the continued use of words like improve, improvement, and their lexical adaptations or superlatives of verbs serve to constantly remind the reader of this overarching theme. For example, the introduction to his discussion of productivity uses "improving" or "improve" six times on one page alone (Meisel 41). These lexical choices characterize Lee's manifesto as well. But more than that, his introduction, especially his definition of biohacking, starts off the book with a very clear promise: biohacking is meant to maximize, optimize, improve, or prevent. Sticking with this promise, his first part begins with a reference to the age old "obsession" with longevity, the basic desire to live as long as possible. It is this age-old desire that is turned into a promise in the first part of the book: a happy, productive, extended lifespan (Lee, n.p.). The general desire for - and cultural obsession with - a hacked, healthier or more efficient brain carries this promise over into the next two parts. This promise not only positions the human body as inherently deficient but also offers a solution to this lack: to use the tools of biotech to optimize the body.

Optimization for Lee means having a "competitive edge" over the general population. In his introduction he writes: "There is nothing in this book that will cure cancer or turn you into a superhero. What there is however, is a range of pharmacological and non-pharmacological tools to enable you to get an edge over the general population." (Lee n.p., my italics) Optimization is not a form of human extension, as trans- or post-humanist would have it, but a process of making your body work more smoothly, giving you more energy, brain power, better moods, a longer and healthier life. It is not in the grand gestures that the potential lies but in the little steps that make life better, more fulfilled and happier. His approach is that already possessing knowledge gives you that 'edge' over the rest of the population. If you take the right measures, you can reap not just the benefits that knowledge brings you but also practically apply that knowledge "to become not just normal but better than normal" (Lee, n.p.) - to attain the American ideal delineated by Elliott, to become Better than Well. This discourse of optimization is enmeshed with a latent rhetoric of responsibilization. Apart from his generalized advice, Meisel, for example, often uses normative language: He claims that he included three basic strategies that work for everyone in his chapters. Therefore, everyone "should adopt" them because they will "lead to healthy improvements" (6). Similarly, Lee lists "compulsory" supplements such as Omega 3 or Vitamin D, which are beneficial for everyone and should be part of your daily regimen. This type of prescriptive discourse promotes the idea that it is your own fault if your body machine fails you and that it can be amended by individual actions.

Another common thread in Lee's and Meisel's 'studies' is the wholesale belief in scientific solutions. Both follow a science-based approach: They repeatedly stress the wellresearched nature of their 'hacks' and their scientific foundation. While Meisel often generalizes in the name of shortness, he nonetheless frequently refers to the "abundance of scientific research" (33) that underlies many of his claims. For Lee, the scientific foundation is already stressed in the subtitle and remains a recurring theme in all his three parts: "cutting edge", "neuroscience based", "strong research backing," "proven efficacy 
or safety," "solid scientific backing" are only some of the phrases he uses to underline the strong background in science and research he claims for his hacks. Moreover, both also refer to academic publication platforms such as PubMed as a resource to help you make "informed decisions" (Meisel 64). By highlighting the "research backing" of their arguments, they take part in a process of authentication through science. Citing scientific sources and stressing the scientific basis of their claims allows them to transfer some of the perceived authority of science to themselves, thereby buying into the cultural ideology of scientism. ${ }^{12}$ The importance they ascribe to scientific validation is affirmed through labels such as "powerful" or dramatic. Lee often describes his solutions as the "most powerful," similarly Meisel also often uses phrases like "dramatic results" (in the positive sense). This form of dramatization or hyperbole is used consciously by them to convince their readers of the validity and necessity of the techniques and tools they present. But by doing so they also linguistically connect science to power: Science leads to powerful effects, powerful solutions and dramatic positive changes. It is thus implied that the ultimate source of power over one's biology and body lies in science. The scientific method is the one that will grant people authority over this seemingly last caveat of uncertainty.

Meisel and Lee stress the importance of granting or providing the type of information summarized in their guides to interested audiences on a "quest for knowledge" (Meisel 64). As Lee writes in the last sentence of the introduction, "information is power" (n.p.). Access to information gives you the power (of science) to shape your body and the tools to make informed decisions. For Lee, thus, information, knowledge, and understanding are key to biohacking: it not just enables the individual to better assess their own bodies and their status quo, but it also allows them to find 'fixes' for their problems and give better qualified input to their healthcare professionals.

A common experience I have when providing consulting to clients is that they often have a huge appetite for learning more about their own individual neurochemistry. You can't fix what you can't identify and in this context it means that when you understand what is happening inside your own skull a little better, it enables you to tweak and tinker using a range of substances and Behaviours. (Lee n.p., my italics)

Access to information and a deeper understanding of biological processes for him are a necessary feature of self-directed choices and active patienthood. Serious efforts of biohacking necessarily need to involve "at least some understanding of what is occurring under the hood" (Lee n.p.). In order to manipulate individual biology - "to tinker outlets in Germany began to report about 'fake science,' meaning scientific studies published in sub-par scientific publications (for high fees) without peer review or the necessary quality control: studies published and amended to fit the buyers' criteria, studies that exaggerate successful animal trials to full-blown cancer miracle drugs, studies that cannot be replicated and so forth. The existence of such studies - and the infrastructure that makes them possible - is also connected to the pressure to publish in many academic disciplines. Still, when communicated to the public 'fake science' has the potential to be highly misleading for the public and patients. If debates about these practices persist, they might also shine a new light on scientism and the validity of scientific claims. 
and optimize" (Lee n.p.) - a certain understanding of the underlying processes and systems is necessary. Clearly, the problem with such a view is that it limits the range of those who can potentially use biohacking to those able to understand (and thus use) the information they are now granted access to. Such limitations potentially create new discrimination based on levels of education and (scientific) literacy.

This focus on self-education and knowledge is much more pronounced in Lee than in Meisel. He wants his book to "serve as a tool" that makes these complex topics "more accessible and entertaining" (Lee n.p.). Lee positions himself in the role of a translator or mediator:

Also, anyone familiar with neurology and pharmacology will have to forgive any gross oversimplifications. There are no shortage of tomes dedicated to the minutiae of pharmacology and the brain which are impossible to understand for those not formally trained. The goal of this section is to successfully translate that information via simplified analogies and generalizations to enable others to understand what is often referred to as the most complex structure in the universe - the human brain. (n.p., my italics)

His self-ascribed role is to translate scientific findings into actionable information, even if that entails generalizations and analogies that simplify the processes. So while Lee rightly acknowledges the complex process of translation and the role that translators play in it, his focus on scientific findings sometimes implies that there is a form of 'insider knowledge' that only those in the know possess and can use. This is a trope often found in self-help literature as well. As part of those initiated, in his view, he is there to provide the reader with that knowledge. This view seems to perpetuate an arbitrary form of distinction between science and the rest of the world that so many in the sphere of DIY biology and biohacking denounce and want to tear down. Still, the whole book could also be seen as an attempt to increase the readers' literacy. Lee consciously repeats certain key points - in the beginning he makes this obvious by stating that repetition is key to understanding - and often emphasizes that active biological literacy is needed to biohack successfully.

The idea that knowledge is power and that figures like Lee and Meisel are needed as translators and mediators of scientific knowledge, who turn 'high-brow' scientific findings into actionable information for their clients, seems to reinforce one of the Rose's mutations cited above: Lee, Meisel and other authors of DIY biohacking guides and self-help books can be considered as part of the new class of experts of the soma. They are needed to guide and inform the uninitiated, like genetic counselors in Rose's account. Their expertise lies not just in their understanding of biological pathways and scientific discourses but also in their own experiences. Most of them are DIY experts that now use their own journeys of biohacking as a resource for consultations, publications and speaking engagements. Borrowing from religious rhetoric, they are the new 'prophets' preaching to their clients and the general public about possibilities to transform their bodies and lives. They tap into latent cultural desires and fears - for health, wellbeing, maximization and of corporeal vulnerabilities - to sell their expertise. To fulfill the broad promises they make, they draw on cultural discourses of plasticity and scientism to underpin their claims of science-based modulations. This is not to negate the potential to increase the scientific literacy of their readers or even their own innocent desire 
to impart knowledge and help others, but rather to stress that here we witness the rise of new "economies of vitality," that the next case study will elaborate on.

\section{Case Study II: Biohacking on the Web - The Example of Asprey's Bulletproof}

For cyber-hacking, the maker movement and DIY bio, the Web has become one of the most important information and community-building tools. Used as a collaborative space of exchange, a cheap, readily and world-wide accessible platform for discussion, the sharing of experiences and 'how-to'-guides, it can rightly be considered as one of the preconditions that facilitated the rise of DIY biology. No wonder, then, that is has also become a playground for the types of body-bio-hacking that are the topic of this chapter. For them, the Web has become a platform used for the dissemination of information and for edutainment but also a virtual space populated by online shops and direct-tothe consumer marketing. ${ }^{13}$ More than the printed self-help guides above, the blogs and websites under consideration in this case study are geared toward commerce. ${ }^{14}$ Here we are not in the collaborative, Web 2.0 space evoked by other DIY practices, but in a use of that space which emphasizes authority - of information, science, the authors and experts behind the blog - to sell services, products and information. As such, it is a highly competitive space, in which 'ranking first' on Google means converting more customers and maximizing profits. Their immediacy, visuality and accessibility make such webpages and blogs perfect examples of contemporary 24/7, digital knowledgesociety. They incorporate, even amplify, social and cultural trends, their fast pace allows them to much more flexibly react to the demands of their audiences, their modest outlay means that also 'niche' or countercultural trends find representation and can increase their coverage.

One of the most widely known examples of such a combination of education and commerce is Dave Asprey's Bulletproof. Dave Asprey, a prominent representative of the body hacking movement, became famous for his signature Bulletproof coffee, a special way of brewing coffee involving amongst other things large quantities of grass-fed butter and oil. This combination of coffee and fats was cleverly marketed and widely reported on also by celebrities and the media. From there on he expanded his business, gradually including guide books - his Bulletproof diet self-help book (2014) and antiaging manual Super Human (2019) are also ranking high on Amazon - and a podcast, supplements and "fatwater," prepackaged Bulletproof -style cold coffee and training machinery. Recently, Bulletproof also started to open cafés in Seattle, Santa Monica and Los Angeles. While the hype around Bulletproof has calmed down in the last years, the success of this brand is one of the most prominent cases of consumer-oriented, DIY

13 All of them use some of the most common online marketing techniques popular today. Despite being avenues for education, their blogs, for example, double as forms of content marketing used to draw people toward the web shops and generate potential costumers (leads). They also run consumer-specific advertisements online.

14 A short note on terminology: While originally 'weblog' was used to denote such informational, journalistic webpages, I will use the truncation 'blog,' that is much more commonly used today. 
biohacking of the body. The wide reach has popularized biohacking among a broader audience and, to a certain degree, attached a name and a face to the practice.

Already the name of the brand is telling: "bulletproof," free from harm, invincible. It takes up a common cultural metaphor, employed also in other pop-cultural formats. ${ }^{15}$ Being bulletproof means that you are strong; you, your body and mind, without failures or damage, impenetrable and, almost, death-defying. The name itself, thus, is a promise, the emblematic goal of the biohacking practices endorsed by Asprey and his team. The Bulletproof Blog then introduces the reader to ways and means through which this goal can be reached. It consists of a collection of 'How To's' - how to stop sugar cravings, eat for a stronger brain, strengthen your willpower muscle, find your ideal carb intake, amongst other examples - tips, lists, recipes and other pieces of advice. Many of these mimic forms of 'journalism' commonly found in popular magazines, such as stereotypical women's magazines filled with advice on finding a partner or losing weight. The themes of the Bulletproof guides, however, do not just include diet and exercise but also common biohacking topics such as grounding/earthing, supplements and performance-enhancing drugs, as well as more mundane aspects such as social connection and parenting. Interestingly, many of the pathways or mechanisms people are advised to modify are similar to those described by Lee, such as BDNF, the MiracleGro of the brain. ${ }^{16}$ It is no surprise, then, that also the Bulletproof Blog has a pronounced focus on explaining the biology behind their biohacks, often in a molecularized form that focuses on biochemical pathways and individual molecules or vitamins.

Following this logic, for Asprey biohacking is about taking control of your own biology. His view combines the idea of controlling your body and your biology with attempts to upgrade, enhance them to become "superhuman." An infographic about "What is Biohacking?" on the Bulletproof Blog offers a concise, dictionary-style definition: "Biohacking: (verb, noun) (v) To use science, biology, and self experimentation to take control of and upgrade your body, your mind, and your life. (n) The art and science of becoming superhuman" (my italics). In the text accompanying the graphic, the first part of the definition is changed to "(v) To change the environment outside of you and inside of you so you have full control of your biology, to allow you to upgrade your body, mind, and your life" (Asprey, "What Is Biohacking", my italics). In this definition, the self as agent is responsible for creating optimal conditions inside and outside, mind and matter are

15 Some pop-cultural examples from the last few years include two highly popular songs - La Roux' "Bulletproof" (2009) or David Cuetta and Sia's "Titanium" (2011) - and, taking up the cultural imaginary created by superheroes (cf. Chapter 3), Marvel's Luke Cage, a web TV series produced for Netflix (2016/2018). Luke Cage is a literally bulletproof, African-American superhero, whose powers are the result of human experimentation. Cage, of course, also takes on a host of different meanings in the context of debates around police brutality against African-Americans and is premised also on a culture in which death by bullet is an everyday occurrence. This superhuman theme is also explicitly taken up by Asprey's definition of biohacking.

16 BDNF - brain-derived neurotrophic factor - is a growth factor that works on neurons in the central and peripheral nervous system, encouraging the growth of new neuronal connections and supporting existing ones. As such, it is connected to long-term memory, neurogenesis and neural development (Spektrumverlag). 
subject to conscious decisions made by the self. A similar definition in a guide to "Going Bulletproof for Beginners" on the blog enriches this standpoint with an emphasis on the personalized and experimental nature of biohacking: "Biohacking (verb, noun): (v): changing your environment from the inside-out so you have full control of your biology; using your body as your personal laboratory, finding the exact hacks that work for you. (n) The art and science of becoming superhuman" (Asprey, "Going Bulletproof", my italics). The more attempts at definitions one considers, the more persistent Asprey's preoccupation with the notion of control seems to get. In an interview with Tim Lewis for The Guardian in May 2017, for example, Asprey is asked about his definition of biohacking: "Biohacking," he claims once more, "is the art and science of changing the environment around you or inside you so that you have full control of your own biology" (T. Lewis, my italics). His own attempts of biohacking, he says in this interview, where less about improving his health, and more about "having control of [his] own biology" (T. Lewis). Control seems to be a crucial factor for Asprey that can also be read as a promise to potential clients: In a time where control over many aspects of one's life is decreasing and vulnerability abounds, the body and its biology become the one thing that people themselves can have control over, facilitated by technology and science. Through biohacking, helplessness is countered with power, subordination and subjectification with control. At the same time, this emphasis on control stands in stark contrast to those early associations with hacking - unlawful, unregulated, covert, countermining. Hacking, in its original form, undermines control, while here the same terminology is used to express the opposite. We might thus ask whether these uses are more marketing than substance: Similar to Lee and Meisel, the use of hacker terminology might be a process of linguistic framing (as modern, technological, scientific, experimental), while the emphasis on control promises to fulfill latent cultural desires.

According to the same infographic the "hackable" entities in human life include mind, body, and lifestyle. All can be optimized in Asprey's view to think faster, get stronger, live smarter, and be healthier ("What Is Biohacking"). More concretely, the types and techniques of 'hacking' Asprey includes in his definition range from supplements, meditation, mental attitude, control of the environment, to the tracking of inner processes, changes in diet, control over sleep, and fitness regimes ("What Is Biohacking"). Similarly, the guide to "Going Bulletproof for Beginners" recommends Bulletproof articles as starting points that look at diet (Bulletproof Diet and Bulletproof Coffee), ketosis, healthy fats, medium-chain triglycerides (MCTs), intermittent fasting, sleep, stress management, detox, supplements, and nootropics (Asprey, "Going Bulletproof"). All these techniques, as Asprey prominently includes in the infographic, "use empirical evidence" to provide "evidence-based upgrades" ("What Is Biohacking"). This definition of biohacking, overall, covers a similar territory as those given by Meisel and Lee: they include physical, mental and environmental factors and focus on science and experimentation. What sets Asprey apart is his pronounced focus on power and control over biology and nature. 
While the Bulletproof coffee and diet are disputed among dieticians and scientists, ${ }^{17}$ like Meisel and Lee, Dave Asprey sells his brand on the promise of its scientific basis and accuracy. In the guide to "Becoming Bulletproof" for example, Asprey claims that he is using the latest research to guide his selection of hacks: "I work on the cutting edge of research and theory, with some of the stuff I do being in the early stages. Years of research help to determine what's safe enough to try it on myself, and I continue to use and refine what works." ("Going Bulletproof") Also in the interview for The Guardian, he feels compelled to emphasize how his approach is "very science-based:" What he does is using the scientific method of observation, hypothesizing, and testing. "The stuff I'm doing isn't just like flip of a coin - it's a hypothesis based on reading thousands of papers about biochemical pathways" (T. Lewis). Here he draws on the common belief in scientific solutions and the trust of science in society. Often the Bulletproof Blog includes references at the end of a blog post, mostly to scientific journals such as Nature, NCBI, PLOS, or Wiley. These serve to validate the given information and make sources transparent to the reader - a form of authentication through science. Something similar happens in the Guardian interview with Asprey. Asked about criticism of pseudoscience that his methods receive, he answers: "So I welcome all the critics: all they need is to turn the book over and look at the quotes on the back from the most successful and well-respected brain doctors and anti-ageing doctors." The "most successful" and "wellrespected" doctors are used to transfer some of their perceived expertise and authority onto his methods, after all medical personnel is still regarded highly in culture. He overtly evokes figures of authority to bolster his own credentials. But Bulletproof also provides a more emotional, community-oriented validation of its methods: A community of like-minded individuals striving for a similar goal is evoked, often through customer experiences or customer references, as Asprey also includes in his infographic. A common tool in the online marketing sector, they try to sell by making biology and hacking more personal, validating its success through the experiences of others.

As we have already seen above, science and self-experimentation are crucial in Asprey's attempts at a definition of biohacking. The infographic deals with the experimental nature of biohacking by including a section on the "process" endorsed by this method. The focus is on " $\mathrm{N}=1$ " experimentation, the readers' "own experiment." Grounded in their own biology ("test your biology"), readers are encouraged to "pick goals" and "track results" to "see what's working" for them (Asprey, "What Is Biohacking"). The wording here also highlights the do-it-yourself approach used by Asprey: DIY experimentation, following the scientific method, is proposed as the best way to optimize wellbeing. The individual, however, clearly is responsible for taking the correct measures on this road to optimization. As is typical for DIY approaches, the focus is not just on the end result, but also on the process- and project-oriented nature of the endeavor. The end goal of this process, in the Bulletproof universe, is to "upgrade" the human body to become "superhuman," as his many definitions of biohacking illustrate.

17 For example, journalist Julia Belluz includes a clinician's view into her report on how the Bulletproof Diet is "everything wrong with eating in America" on Vox.com (2014), linking Bulletproof to other popular fad diets, which promise consumers more than they can deliver while being overly prescriptive and condemning of other diets. 
The optimization potential of the human body is stressed on multiple occasions on that infographic: To do so, Asprey uses key phrases such as "get stronger + think faster + live smarter" ("What Is Biohacking"). As we can note, they once more align with neoliberal ideals of competition and performance. What these and similar expressions have in common is a continuous use of comparatives and superlatives. The goal is always to somehow be more than you already are. This is also emphasized in the guide to Bulletproof: "Upgrade your life and get better at everything. No matter your weight, your background, your family or work situation, I believe you can upgrade your body and your brain to perform better than you ever have." (Asprey, "Going Bulletproof")

The most important step in that process, Asprey writes a few lines down, is the "belief that you can change" ("Going Bulletproof"). The general assumption behind many of his molecularized, vitamin- and supplement-based 'hacks' is that of a certain plasticity of the body and brain. Modification, for Asprey, is possible. Instead of older assumptions of genetic determinism or critical periods in brain development, Bulletproof endorses a view of the body whose very fundament is its ability to change. Once more, Asprey evokes his personal experience to underscore this argument: "I can tell you firsthand that you're not condemned to live with the body and brain you were born with." ("Going Bulletproof") To use Emerson's words, the body and brain are "not fixed but fluid" ("Nature 1836" 80) - nature is not a prison you cannot escape but a playground of possibilities. Instead of being subjected to your body, you can take control and change it, at least if you believe that such change is possible. Here Bulletproof combines a rationalist claim based on current scientific research with an affective appeal. The reader is empowered to take control into their own hands and create the body and life they always dreamt of.

It should not be disregarded, however, that the information provided by Bulletproof and other similar endeavors do not primarily serve the function of promoting self-education and increasing literacy - even though they might serve this purpose well. ${ }^{18}$ Rather, they are 'entry points' for visitors of the webpages that satisfy a latent need: for information and knowledge, an open ear, tools to help themselves. The goal is to turn an interested reader into a customer. For Asprey, this process is rather straightforward. His online shop is integrated into the blog, so that customers can shop in one stop. Customers can order coffee products, foods and drinks, protein powders and bars, as hacked/Genius Labs is a similar endeavor: Presented as an information blog, Selfhacked.com makes its profit by selling electronic guide books and diagnostic services as well as providing (probably paid) links where you can buy supplements. This page offers the reader information on single nutrients, natural substances, herbs, drugs and nootropics. But more than that, the reader can familiarize themselves with the science of biohacking - including descriptions of biochemical pathways - or access specific guides on medical conditions from autoimmune diseases to mental health or "healthy interventions" for enhancement, diet, sleep, stress and detoxification. The name, thus, is telling of its approach: the self hacking its own biology, an overt reference to the DIY culture of biohacking. But Selfhacked also wants to offer a different route for those who feel unheard and neglected in the medical establishment by providing them with information and counseling on how to "fix" their own medical problems. Their approach clearly draws on the discourses of active patienthood and patient empowerment so prevalent in medicine during the past few decades. 
well as Bulletproof's signature supplements, the books and merchandise. With this goal in mind, one can reasonably argue that the rhetoric of empowerment, modification and optimization in his other 'channels' is also used to responsibilize the reader in the name of consumption. Following the 'self-help' scheme of problem-solution, readers are confronted with a real or presumed problem or deficit, for which Bulletproof cannot just offer context and information but also the tools to solve it. People are instructed to follow certain practices, take specific supplements or make changes to their lifestyle for which the shop offers everything they need to succeed. Hacking the body - here integrally linked to optimizing its status quo - becomes a commercialized enterprise: The DIY hacker is necessarily a consumer. It is this consumption-oriented form of DIY that has moved outside of the niche into mainstream markets. The logics behind it, however, are still the same: You can and even should 'do-it-yourself.' It is still the DIY patient as health consumer, but this time they are not limited to solutions for medical problems but take part in the contemporary race for self-optimization. It is here that DIY and the marketplace come together to create new "economies of vitality" that extract commercial value from the age-old desire for human perfection and the contemporary belief in physical malleability (Rose, Politics of Life 5-7). These "economies of vitality" today have largely been displaced into the sphere of the digital: The possibilities of the Web make them more accessible and more encompassing than previously possible.

\section{To Summarize}

It is very much the ideology of the active, informed patient that is required and perpetuated in these examples. Individuals are expected to be highly involved in the decision making around their own bodies. The case studies in this chapter show that this ideology has today reached a new degree of commercialization, leading to an abundance of products marketed to the 'patient' and new forms of professionalization. But this form of DIY also exhibits a strong demand for access to information, knowledge and tools. Both are brought together by drawing on popular discourses of individualization: Stressing the importance of individual solutions makes it possible to both fulfill an ideal of patient education and empowerment and commercial interests as service providers. Information itself is not enough, you might still need someone to help and guide you on your individual journey, the logic goes. Initiating a personal connection through referencing their own experiences and those of others allows the new experts of the soma to sell their services and bolster their credentials. Biohacking, in these case studies, becomes a tool of (self-)marketing and economic exploitation. The self, once more, is responsibilized to optimize its own corporeal status-quo to enhance its productivity and wellbeing. Social and structural factors that impinge on individual possibilities are all but elided. There is, however, also room for a spirit of collectivity: As the example of Bulletproof shows, it is in the sphere of the online world that connections are made, (virtual) communities created, allegiances to a cause formulated. People present themselves and define themselves as part of a larger group, a community that shares experiences, as the hashtag "\#iambulletproof," promoted in social media, serves to show.

The goal of this 'journey' of biohacking the body is two-fold: Self-knowledge of one's own biology and the optimization of its current state of being. This type of hacking, 
necessarily, promises to have material results on the body: to be and feel better, fitter, healthier, stronger, happier, live longer, enhance cognition, treat depression and anxiety. Biochemical pathways and the molecular composition of the body are expected to be changed and 'supplemented,' with overarching effects on the whole organism. Still, whether the promoted effects actually occur and are caused by the marketed interventions remains unclear. It is noteworthy that much of what Lee, Meisel and Asprey reframe as 'hacks' are actually old and well-known practices such as yoga, exercise, or vitamin supplementation. Using a new language integrates those practices into a science-based and solution-oriented outlook on life. 



\section{A Pill for Every III - Drugs and Supplements}

Contemporary self-help practices in DIY biology are commercialized enterprises: At their core are the new class of experts of the soma - the lifestyle coaches, self-made hacker gurus and start-up companies - which have played a big role in constructing new digitalized "economies of vitality" around the self-directed optimization of the human body. Foundational for these strategies is not just the monetarization of information and access but also a very much material thing: Pills, supplements, powders, superfoods. Dave Asprey's Bulletproof, for example, sells signature supplements with such telling names as "Brain Octane," "NeuroMaster" ${ }^{\circledR}$ or "Unfair Advantage" - names that already imply power, control and the famous 'edge' over the competition. ${ }^{1}$ These pills and capsules are marketed as tools to help individuals reach optimum performance and increase their overall well-being. Supplements, however, are of course nothing new, but rather an old, consumer-friendly practice of self-treatment and self-optimization: daily regimes of vitamins, natural remedies and herbs are a natural part of day-to-day life for many Americans. ${ }^{2}$ In this chapter, however, I want to move away from simple vitamin supplements towards applications like Lee's, who also thematizes the use of drugs and biochemical compounds to 'hack' the human body and brain. I will look at the possibilities to use drugs and supplements as DIY technologies: Drugs and supplements are probably some of the oldest forms of self-medication, but today can be seen as inhabiting an ambiguous position between treatment and enhancement while at the same time being wholly incorporated into the ideology of the patient-consumer.

What hides behind these names are extracts from the Arabica coffee fruit (NeuroMaster ${ }^{\circledR}$ ) proclaimed to help memory and cognition; a medium chain triglyceride - a type of fatty acid - distilled from coconut oil (Brain Octane) said to improve gut and brain function; and a combination of Active PQQ ${ }^{\top m}$, Coenzyme Q10 and Brain Octane oil (Unfair Advantage) marketed as boosting mitochondrial efficiency to give you more energy (Bulletproof Executive).

2 A widely available but highly controversial supplement that has saturated the American TV market with its commercials in the past 10 years is Prevagen, advertised as boosting memory function and preventing memory loss associated with aging. In the past years, government authorities have started to proceed against the manufacturer for fraudulent and deceptive advertisement. The clinical evidence for Prevagen's safety and effectiveness that was presented and marketed by the manufacturer is inconclusive at best, cf. Maggie Fox. 
Many researchers have come to the same conclusions: medications, pharmaceuticals, 'drugs' are omnipresent in contemporary American culture. ${ }^{3}$ Some of them are readily available in the aisles of pharmacies and department stores whereas others have to be prescribed by a doctor and collected from a pharmacist. What both prescription and non-prescription drugs point to is a normalization of pharmaceutical interventions: Today, there is a pill for every ill. This impression is reinforced by the prevalence of direct-to-consumer advertising. The US is one of the few countries in which not just non-prescription drugs but also prescription drugs can be marketed directly to the consumer. Especially early psychopharmacological drugs like Pacil, Xanax, or Prozac were marketed aggressively to the public, with similar marketing campaigns for today's attention deficit hyperactivity disorder (ADHD) drugs Ritalin and Adderall in the 1960 s and 70s. Controlled by the Federal Food and Drug Administration (FDA), these types of advertisements necessarily have to fulfill strict criteria. Nonetheless, they also represent a distinct form of public communication with and empowerment of individual patients to take control of their medical decision making. As such they take up and perpetuate the ideology of active patienthood and informed choice. Today, many doctors commonly find themselves confronted with patients who actively request a drug they have seen in an advertisement. While this has contributed to a reversal of power roles in the medical encounter, these advertisements cannot only have pronounced effects on the cultural perception of disease and treatment but also be misleading in their claims.

Pills, today, are not just used as treatments: More and more, every function of the human body we perceive to be somehow deficient can be alleviated with some form of a drug, so that also healthy bodies are subjected to pharmacological interventions to better conform to our desired lifestyles. Such lifestyle drugs, like the rebranding of the contraceptive pill in the 1990s, often occupy a space of ambivalence between their marketing and their medical value. ${ }^{4}$ Psychopharmaceuticals are an example of this ambivalence, being marketed as forms of lifestyle drugs but also used to treat very much real conditions with a high degree of suffering. In the 1960s Prozac, a tranquilizer drug used to control anxiety, was sold as a drug that would help patients adjust to the new pace of American society: In descriptions of patients, Prozac became a positive force, a drug that allowed them to remake themselves and take on a new personality as a person without depression (Stevens 281-85). Psychopharmaceuticals, in such cases, are positioned as means to "become our true selves" - the drugs and chemicals themselves good sources that also look at the omnipresence of drugs in American culture. A very timely example is the opioid crisis captivating the US at present: Since 1999, illegal and prescription opioids - prescription pain killers, heroin and synthetic opioids - have caused a high rate of abuse, addiction and overdose deaths in the US, so much so that in 2017 a national emergency was declared.

4 The pill, of course, was originally intended as an answer to a common medical need of women: to control their menstrual cycle and fertility. By doing so, it had widespread cultural effects. The drug, heavily marketed by the pharmaceutical industry, has given women more control over their biology, their family planning and thus their life, permitting greater sexual and social freedom (Stevens 280-81). But especially since the 1990s, the contraceptive pill has also been marketed as a 'fix' for more or less cosmetic problems: acne, menstrual bleeding, premenstrual syndrome (PMS), mood imbalances - essentially being turned into a lifestyle product, cf. Watkins. 
becoming "part of our very identity" (Stevens 290). Here he takes up an argument made by Emily Martin in 2006: Even though discourses around drugs are often permeated by ambivalence and contradictory feelings, she argues, Americans are more and more becoming "pharmaceutical persons" that not just simply consume drugs in unprecedented numbers but also increasingly incorporate these drugs into their sense of self, their personality ("Pharmaceutical Person"). As people define their dis/abilities more and more in biochemical terms, also drugs become incorporated into their view of the self. In the US, Martin contextualizes, this trend is also facilitated by the cultural and social environment of free markets and little social security: In such a setting where individuals are responsible for their own success or failure, drugs and other forms of enhancing mental capacities can easily become necessary means to look after the self ("Pharmaceutical Person" 274). Often, the image is that a pill will make the consumer "a better person, an enhanced person or, more precisely, more like the person they really are" (Martin, "Pharmaceutical Person" 275-76). This is enabled also by commercial narratives of psychopharmaceutical drugs which often propagate the restoration of the (former) self through drugs: What is sold to the patient is a dream of control, of modulating your biochemistry to feel like yourself again and get your life back (Rose, Politics of Life 214; 100-01). Psychopharmaceuticals can thus also be regarded as a "technique of the self," a means to change and shape the self according to one's own ideal (M. Butler, "Spiel" 88). Through their framing as lifestyle products, drugs to enhance or control normal parts of human biology have become an ordinary part of everyday life. As such, Stevens argues, drugs are not just treatments but biotechnologies that play an important role in "determining who we are, what we want, and how we live" (290). They have effects on how we negotiate the capabilities of the human body and its plasticity and are imbricated in how societies valorize the interests of society versus individual freedoms to optimize. Pills have become a means to change who we are as humans, with material, social, and cultural effects.

Drugs are products of society and culture as well as science and technology, and as such are implicated in definitions of disease, commercialization, bodily control, and the re/designing of life: in terms of disease, they help define what is normal and disordered and thus help create the disease they treat; as products they are part of the consumer market for the sick and the healthy; they give us a powerful tool with which to manipulate and control the body and biological functions according to ideals of physical plasticity; and the ability to use them to control our bodies according to our desires means we can also use them to redesign our lives, to choose a lifestyle and actualize it through the use of drugs (Stevens 286-90). But they also are implicated in questions of access, distribution and justice: On local and global levels, access to drugs often is differentially distributed in and between societies and nations with severe consequences for the health and life chances of individuals. Drugs thus are more than their material form. They help shape culture and our notion of the self, with implications not just for the individual but also on a collective level. What we should never forget is that drugs are not just lifestyle enhancers and molecular playthings: They can also be lifesavers, needed and necessary treatments, whose availability can be highly restricted.

The case studies under consideration here look at drugs and pharmaceuticals in the context of DIY biology and medicine in different ways: Case Study III examines the rise 
of stimulant use by healthy individuals to reach personal goals through the lens of a 2018 Netflix original documentary, Take Your Pills. Case Study IV contrasts this commercialized form of DIY with a more political, maker-based approach in its consideration of the EpiPencil (2016) hack by the Four Thieves Vinegar collective. This juxtaposition shows two different modes that DIY is taking with regard to drugs: first, consumer decisions to take drugs to modulate their life and bodily performance according to their liking and second, a creative intervention (or DIY solution) to make drugs cheaper and available for everyone, to raise awareness about rising costs and socioeconomic barriers of access. Indeed, my analysis has revealed agency and access as overlapping preoccupations: Who is allowed to use what types of drugs for which purposes? Whose use is culturally sanctioned and whose is not? Who can afford drugs and when is access hindered? These questions, also in the case studies under consideration here, are to a certain degree of course intersecting with considerations of class: in the third case study a certain level of income is needed for this type of drug use, in the fourth case study some people are excluded for economic reasons. The former can lead to new forms of stratification, while the latter could provide answers not to the questions posed by the former but to medical segregation, globally and in insurance markets like the US.

\section{Case Study III: Take Your Pills, Become Limitless - The Stimulant 'Epidemic'}

The drug "Soma" in Aldous Huxley's Brave New World (1932) is probably one of the most cited examples that demonstrates, in literature, the fascination humans have with pharmaceutical modulations of the body. In Huxley's dystopian imaginary, Soma is used to calm, sedate and distract the population from the flawed system they live in. Such dystopian examples stand in marked contrast to seemingly utopian, contemporary fantasies of the 'brain pill,' that has been part of the cultural imagination, not least since the blockbuster Limitless (2011). ${ }^{5}$ Such elusive brain pills typically allow the user to utilize their mind 'to its full potential.' It is a form of instant and easy optimization, whose incorporation into the cultural imaginary reflects both current biological possibilities and cultural trends of performance, efficiency, and optimization. In reality, of course, until today no such magical brain pill exists. But the culturally entrenched human fascination with brain modulation has led to 'real world' applications of biotechnology in the use of nootropic, meaning cognitive function improving, substances and the widespread $\mathrm{ab} /$ use of stimulants used to treat medical conditions such as ADHD, most famously Ritalin and Adderall - "the drug of our time" (1:23:39), as Dr. Wendy Brown puts it in Netflix' documentary Take Your Pills (Klayman). ${ }^{6}$

In Burger's film, the use of a new nootropic drug turns the protagonist from an unsuccessful writer into the complete opposite: a successful investor and broker. The drug, it is claimed, allows him to use the full potential of his brain and turns him into a perfectly functioning capitalist. This movie is one of the best-known examples of nootropic drugs in science fiction and certainly has spurred on the fantasy of brain enhancement.

6 The title for the documentary is inspired by one of the cases it portrays: Jasper, a college student who was diagnosed with ADHD in third grade and has since then been "on" Adderall, is using his ambivalent, critical relationship with the drug as input for his art. He has started to use the phrase "take your pills" in many of his art works, inspired by the many times his mother asked him whether 
Directed by Alison Klayman and released in 2018, this documentary details the use and abuse of stimulants like Adderall and Ritalin on college campuses and in the work force. Take Your Pills shines a light on the rise in stimulant consumption in the US: It looks at these pharmaceuticals from the perspective of those diagnosed and those $\mathrm{ab} / \mathrm{using}$ the drug with or without a prescription. But is also manages to set the current uses into context both by including historical perspectives on the manufacturing, marketing and prescription of the drugs in the past and by including contemporary DIY alternatives such as nootropics and micro-dosing. Underscoring this multifaceted view, the documentary is not told by a narrator but by individual people - experts in the field, researchers, current and former users - and cultural representations, such as clippings from newspaper articles, books, or old advertisements. ${ }^{7}$ College students, people in the work force - especially the technology industry and finance world - but also competitive athletes are chosen as exemplary users. From children to adults, students to high professionals, it is emphasized, the widespread use of stimulants is common. Throughout this amalgamation of voices, the documentary tries to balance positive and negative ones - different 'truths' are allowed to be heard. Take Your Pills demonstrates not just the historical continuity of self-enhancement techniques but also underscores the biosocial community in which it takes place, putting emphasis on the social and cultural reasons behind currents trends to hack the self. Self-optimization - also through use of pharmaceuticals - is portrayed as a (perceived) requirement to remain competitive, while distinctions between treatments and enhancements are further blurred and interventions normalized.

In the documentary, pills have clearly become a lifestyle product: They are used to maintain or achieve a desired lifestyle. This is emphasized already at the very beginning. In one of the first frames, the viewer is introduced to the topic with yellow writing on a black background: "Word hard, play hard" (02:32). This phrase has often been connected to new forms of work especially in the tech industry. The perfect motto for a competitive and hedonistic culture, it shows how the lifestyle these drugs make possible is culturally sanctioned, even required. Their use, thus, is in line with the demands of a competitive market place, a fast paced, image-based culture and society. This social context is also reflected in the overall aesthetics of the documentary: game-like motion-graphics reminiscent of Pac-Man (here a skull eating pills) illustrate the competitive nature; shaky, blurry and colorful images in rapid sequences highlight the speed of contemporary life and give the documentary a pop-art-like, almost psychedelic aesthetic. Visually Take Your Pills serves as a perfect illustration of the increasing, everyday use of pharmaceuticals in the US. Pills, in the documentary, are everywhere: motion graphics of mountains

or not he had taken his pills. Taken over as the title for the documentary, "take your pills" retains some of this critical stance but combines it with a culturally-entrenched appeal - take your pills and all will be well.

7 The documentary also makes use of a lot of pop-cultural references including The Simpsons ("Brother's Little Helper," 1999), Charlie Bartlett (2007), The Bling Ring (2013), and Silicon Valley (2014current). Also Limitless is referenced in Take Your Pills as the first example that brought cognitive enhancement widespread social recognition (Klayman). Already the references chosen for the documentary show how the hyper-availability of Adderall in the US has long made it into the cultural imaginary as a (comic) point of reference. 
of pills, pills dropping through the frame, pills as players in popular computer games are used to visualize what interviewees are describing, inserting the pill into the documentary both as material object and iconic representation.

Pills, the documentary makes clear, are today incorporated, literally, into the sense of self. Some of the users discuss the repercussions their consumption of stimulants had for ideas of the self and personality. Often one of two cases is true: Either the drugs become incorporated into the self - adhering to the narrative of revealing or creating the 'true you' - or they are rejected because the user feels they cloud their true self. One college girl, for example, explains that she would not and cannot think about herself as herself without being on Adderall (02:20): She has integrated the drug into her sense of self and personality, creating if not chemical but psychological dependency. A motion graphic of humanized pills - often an iconic pill with googly eyes - and body parts serve as visual illustrations of this process: The humanized pill is afforded a personality, one that can more easily become part of the user's own. Both the incorporation into the body shown in these motion graphics and the personalization of the pill underline the close but sometimes ambivalent relationship with the user. As a pop-cultural representation, thus, Take Your Pills perfectly illustrates how people become "pharmaceutical persons" (E. Martin): The sense of self is closely connected to the use of the stimulant, ideas of success and personality are entangled in this web of intimate connections. ${ }^{8}$ The ambivalence of these relations becomes clearer in the cases of other users, who explain that they have started to doubt the drug and experienced some sort of identity crisis: For example, their use of Adderall to perform makes them feel like it was not them but they and Adderall that are responsible for their success. For them, being on and off Adderall is like being two different people. Their sense of self is in conflict with the notion of being dependent on a drug to perform in the way they deem necessary. The pill is not seamlessly integrated but used solely as a tool with a specific purpose.

This view of pills as tools to achieve certain ends also illustrates how stimulant use can be considered as a technique of DIY biology and medicine. Of course, in cases in which children or adults were diagnosed with ADHD the medical treatment of their condition is not a form of DIY. However, in its essence, Take Your Pills wants to draw attention to a different type of use, one that is much more self-directed, either by illegally buying a drug (here 'hacking' takes on its shady, illicit connotation), getting a prescription to enhance their performance (this type draws heavily on notions of the patientconsumer and DIY patient), or using other cognitive self-enhancement techniques (relying both on consumption in the new economies of vitality and self-experimentation). The primary idea is that the self is responsible for its own situation and decision making. The role of DIY, arguably, becomes clearer later on in the documentary when the narrative draws a connection to supplementation and micro-dosing as related practices. nificant difference in the results on a variety of cognitive tests between a placebo group and an Adderall-taking test group, except for the answers to one question, as cognitive neuroscientist Dr. Martha Farah explains: "Do you feel the pill you took today enhanced your cognition?" (1:05:301:05:50). Stimulants, it seems, primarily boost self-confidence and motivation, demonstrating how intimately self-perception is tied to the use of the drug not necessarily its effects (Klayman). 
The term hacking is first mentioned when the documentary turns to "brainhacking" as part of the wider "biohacking" movement. This type of hacking is characterized, as the previous chapter has shown, by a high degree of self-directedness but also self-experimentation. The use of prescription drugs like Adderall is portrayed as an entry point to other forms of cognitive enhancement. Jesse Lawler, host of the podcast Smart Drugs Smarts, describes brainhacking as a "subset of the larger biohacking sphere" (1:04:00) and draws on the cultural imaginary created by films such as Limitless or The Matrix to explain the appeal of "smart drugs" or nootropics. ${ }^{9}$ Cognitive enhancement in the area of brainhacking does not necessarily make use of drugs but also other (natural) compounds. The documentary shows a company called AlternaScript which provides its customers with over-the-counter alternatives to prescription drugs, those that you can get "without having to pretend you have ADHD," as Matthew explains (1:07:38). ${ }^{10}$ Interviews with Lucas and Matthew, the co-founders, give the viewer insights into the scene of cognitive enhancements. Both argue that companies like theirs aim to "help healthy people achieve their purpose," to help to turn themselves into the "optimal and ultimate" version of themselves, that, as Matthew claims, everyone is looking for (1:18:15-1:18:31). "Smart Drugs" such as their OptiMind are tools on this road of optimization. People are already taking steps to get to the next level, as Lucas emphasizes, which is why cognitive enhancement is "inevitable" for him: "cognitive enhancement is not going away, it's the future of the human species" (1:09:41). ${ }^{11}$ These steps people are taking are not just nootropics but also forms of "micro-dosing."

Micro-dosing, the documentary shows by including snippets from news coverage, is a practice common especially among those in the tech industries in the Bay Area and Silicon Valley. "Lily," a start-up publicist who wants to remain anonymous, micro-doses every four days with magic mushrooms. ${ }^{12}$ In college she used Adderall from other kids and now feels like LSD and mushrooms are an alternative to reliance on big pharma. She also says that the mentality and culture in Silicon Valley encourages both the use of pharmaceutical stimulants and micro-dosing of drugs. Micro-dosing, for her, is no different than the old-school supplements. Dr. James Fadiman, the second micro-dosing

Derived from the Greek noos 'mind' and trope, 'turning,' the term nootropic is used for substances that (are said to) increase memory or cognitive function. Among them are common drugs from the class of amphetamines and racetams, substances like caffeine and nicotine that work on the central nervous system, as well as dietary supplements (such as Omega 3 fatty acids) or natural herbs such as Ginseng or Gingko extracts, for both of which effectivity is not proven (Berry). As of 2019, the FDA has become stricter in their warnings about deceptive marketing and false product declaration, warning manufacturers and consumers about possible marketing scams, advertising fraud and lack of approval, cf. press release by the Federal Trade Commission on the warning letters sent to some companies, Feb, 112019.

10 AlternaScript, thus, is another example of commercialized biohacking along the lines of those described in the Chapter 6.

11 Here we can also discern transhumanist overtones in the statements of the co-founders, a belief in self-directed evolution as the next step for humanity.

12 Microdosing has also reached popular culture. For example, the Canadian TV sitcom (CBC Television) "Workin' Moms", available on Netflix, mentions the practice of microdosing when one of its protagonists starts working in a PR start-up - where some employees routinely microdose with mushrooms (at work) to boost their creativity (Reitman). 
interviewee, psychedelic researcher and author of a book that brought micro-dosing its current notoriety, explains micro-dosing as using $1 / 10^{\text {th }}$ to $1 / 20^{\text {th }}$ of the conventional dose of psychedelic substances, with "zero psychedelic effects" (1:11:12). Instead, microdosing is said to increase creativity and focus, help combat symptoms of depression, give users more energy and enhance their social skills. This type of self-optimization being illegal or at least situated in a grey zone - is another form in which individuals 'hack' their bodies themselves.

This type of pharmaceutical and supplemental hacking of the body takes place on a molecular level. As Emily Martin says, the "complexity and technical prowess" of pills and drugs "exists at the molecular level." Following this molecularized, reductionist logic, doctors and patients see pills as "precision instruments," molecules engineered for specificity, to work, for example, only on specific receptors, target precisely one specific flaw ("Pharmaceutical Person" 279-81). Like small, highly targeted machines, they are expected to work on and modify biochemical pathways, in the end adjusting the whole system of the body. This discourse of molecularization and molecular modulation necessarily also invokes a certain form of plasticity of the body and brain, an ability to be changed through biochemical pathways and interventions. Take Your Pills illustrates this through a sequence on the molecular explanation of how those stimulants work on the body (21:00-23:00). This stylistically highly interesting sequence explains and visualizes the structures and differences of the substances and the pathways they work on in the brain on a bio-chemical level. The motion graphic that shows how they work on the body imagines the brain as a machine, complete with cogwheels. This is suggestive of a molecularized but also old mechanistic view of the body. Here the documentary draws on and perpetuates common cultural assumptions - a common (visual) language - about the workings of the body, possibly in order to make those more relatable and understandable for the audience. That this type of molecular intervention has material effects on the body is emphasized already in the opening sequence: The first impressions are of users recounting what they experience when taking their pills, their physical symptoms and changes. Some describe how their heart beats rapidly, their handwriting gets neater, they feel a "warm glow" and their mind and body feel like they "come alive" (00:40-00:45). These immediate material consequences, of course, translate into broader effects, such as higher levels of concentration and success in their tasks, and of course also have social repercussions.

For one, Take Your Pills perfectly illustrates how the distinction between treatment and enhancement becomes almost impossible to keep up, as most forms of medico-biological intervention can be used as both. Stimulants used as treatment for ADHD are shown as justified, in some cases even necessary. The documentary emphasizes that $\mathrm{ADHD}$ is real and that some form of intervention has to happen, either through medication or other forms of therapy. Ariana, a college sophomore diagnosed with ADHD, accentuates this point when she rejects the phrase "everybody has a little ADHD" - often used to authorize the use of stimulants - as untrue, even hurtful in that it delegitimizes the power the disorder has over a person's life (39:12-39:20). Not everybody, she claims, has a brain like someone with ADHD and can thus justifiably take corresponding medications. When people without clinically evidenced ADHD are being prescribed stimulants, this might lead to stigma for people who actually need the drugs and disvalues 
their experience of the disease. One of the problems with Take Your Pills, however - and one that has caused a mild wave of public backlash - is that the viewer leaves with the impression that some of those (young) adults taking Adderall with a 'diagnosis' do not really need it to treat a medical condition but rather use their diagnosis for easy access to a controlled substance for their own enhancement.

While the boundary between treatment and enhancement might be fluid, more time is spent on the idea of stimulants as a form of enhancement. Even though many of those interviewed have a prescription, it is implied that getting this prescription might be too easy. For the college students, stimulants are a way to become the perfect student with an active, "Instagramable" social life and perfect grades (21:41). On college campuses around the US "everyone takes it" (03:47). It is a means to get an edge, to become your "best, smartest, fastest self" (6:30), especially in times of desperation. Stimulants become means to optimize performance in the competition around SAT scores and college placements, grades and class rank. This ab/use for enhancement continues in the work force. Adults, the documentary stresses, are now the fastest growing segment of the ADHD drug market (26:54): All the adult users who are interviewed for the documentary acknowledge that stimulants are used to optimize and maximize performance, often in hyper-competitive fields. Nathanael, a software engineer, for example, acknowledges that Adderall is a performance enhancer and that there is "nothing wrong with that" (17:11). For him Adderall is his "jet fuel" (1:03:29) that turns big problems into stimulating challenges. "Peter," a finance worker who wants to remain anonymous, acknowledges that in a work culture such as his, the demand to be a perfect employee encourages stimulant ab/use - but if everyone takes Adderall, you need to take it too to keep up with the competition (54:40-55:00).

For these professionals, taking stimulants is a means to maximize their performance and get an edge over people, to satisfy the demands the job market places on them. ${ }^{13}$ One of the researchers interviewed for the documentary, Alan Schwarz, author of the book ADHD Nation (2016), says that what is developing is not just a dynamic of people using a potentially dangerous drug, but also an "arms race" where people will be coerced to take stimulants if all of their competition does so (1:21:22). This leads to another interesting question, taken up by college student Delaney: She says that for someone like her, who had to work in high school because she is not from a wealthy background, taking Adderall is a form of "leveling out the playing field" but if you are one of "those kids then you are just giving yourself more of a leg-up" (28:12). ${ }^{14}$ It is not just the question of treatment versus enhancement that is interesting here but also that of access: Should drugs be consider a rightful means to reduce differences? Who does and who should have access to them?

The use of pharmaceuticals for enhancement has been termed "cosmetic pharmacology" by Peter Kramer in his 1993 book Listening to Prozac. He applies this term to describe

13 As such, it is, again, close to what Micki McGee diagnosed for self-help books: a tool to improve the self to fulfill the demands of an ever-competitive and unsecure job market.

14 The 2019 college admissions scandal investigated as "Operation Varsity Blues" could be read as yet another example of parents, especially those who already are well-off, trying to get that 'leg up' for their children. 
the use of pharmaceuticals not to treat a condition but to move people into another, socially more desired or rewarded state: Healthy individuals use psychopharmaceuticals not in a therapeutic but in a cosmetic sense. On this basis, Arjan Chatterjee - who was also interviewed for Take Your Pills - coined the term "cosmetic neurology" in 2004 to denote the use of pharmaceuticals for enhancement purposes. Chatterjee develops an interesting idea in his consideration of "cosmetic neurology," namely that physicians might in the future work as "gatekeepers" to the patient's pursuit of happiness (973). More than a decade ago, Chatterjee predicts a future in which patients come to their doctors insisting on using cosmetic neurology as means to pursue their own happiness (92). In this scenario, neurologists and psychiatrists could become "quality of life consultants" whose role is to offer a range of possibilities, set into context likely outcomes and risks, but leave the decision and responsibility to patients because clinical disease markers can no longer guide their care (Chatterjee 972). As the examples in Take Your pills show, in some social circles this type of "cosmetic neurology" is already common. Doctors become intermediaries in a bureaucratic process of prescription, long after the decision has already been made by the patient-consumer that taking a stimulant in the pursuit of their own happiness is the right and responsible decision. This, as the documentary acknowledges, is also connected to social status and class: Having the resources and knowledge to maneuver the insurance and healthcare market makes this type of self-enhancement considerably easier for the individual. Moreover, as Martin has illustrated, pharmaceuticals are also commodities and as such come with a price tag that determines who can buy and use them, especially in the American insurance market ("Pharmaceutical Person" 276).

Fittingly, one of the core messages of Take Your Pills is continuously reinforced: That this stimulant 'epidemic', in fact, is not an individual problem but a social and cultural one. Many of the scholars, scientists and doctors interviewed for the documentary focus on the cultural premises that enable stimulant ab/use but also the social problems that might arise from it. More than a mere problem of the "medicalizing of everyday life" (46:37), as Dr. Lawrence Diller acknowledges it to be, stimulant ab/use is also framed as a symptom of larger cultural issues. Alan Schwarz says that every generation has found a way to enhance their performance, in this case through ADHD drugs. This type of performance enhancement also on college campuses is not new but rather part of a universal desire to succeed (10:00). ${ }^{15}$ So when the documentary goes into the history of amphetamines and stimulants, a large factor in the equation are the cultural circumstances, the connection of pharmaceutical use to American notions of the self and success. Dr. Nicolas Rasmussen, author of On Speed, recounts the history of "pep pills" citing cultural reasons for their huge popularity during the mid-20 th century: "Pep is what Americans want," he says, "it's about drive, ambition, being productive and hardworking and making the sell and getting the job done" (11:05). Getting an edge, being productive and ambitious, for him, is part of an American notion of the self that makes self-enhancements highly appealing.

15 Schwarz recounts that the first article about amphetamine abuse - "pep pills" as they were called - on college campuses appeared in 1937 in Time Magazine (11:00). 
Similarly, Dr. Wendy Brown, a political theorist, argues that contemporary norms tell us to "perform at the highest capacity that you possibly can" for as long as it takes. The problem in her view is that Americans live in a "hyper-competitive order" where "competition is never ending," so that everyone is required to think of themselves as "a little bit of human capital" that can and should be enhanced (05:23-07:20). The anxiety over our human capital value, Brown claims, makes enhancements almost inevitable. Stimulant abuse thus perfectly aligns with contemporary cultural values of effectivity, competition, productivity, excellence and constant availability. By some, the "Adderall epidemic" is described as equaling the Opioid epidemic, the widespread abuse, addiction to and overdose on prescription and illegal pain medications and synthetic variants of them (Klayman). While the Opioid epidemic was acknowledged as a national crisis by the current political administration, stimulant abuse is often less scrutinized exactly because of this connection to desired qualities such as success, achievement, and performance. Even though it is not an immediate threat to life (like opioids are), the underlying ideology nonetheless can become detrimental to the wellbeing of individuals. Anjan Chatterjee, towards the end of the documentary, is pictured as saying that:

...the use or abuse, depending on how people feel about this, is symptomatic of something broader. This highly competitive environment in which people feel compelled to compete beyond their capabilities to get ahead, this kind of focus on material progress and productivity. What's the cost of that and is it a cost we're willing to live with? $(1: 21: 46-1: 22: 20)$

For him, the ultimate question is if this cultural environment and the self-enhancement techniques it bears fruition to have a cost that America as a society might not be willing to live with. Similarly, the question Dr. Brown poses at the end is what is lost in a stimulant-dominated world. For her, this might be the experience of being human with all its ups and downs. The documentary, by allowing that voice to be heard and strategically positioning it at the end of the narrative, thus implies that the ab/use of such pharmaceuticals with its material and social effects has the ability to fundamentally alter what is means - and feels like - to be human.

What can we infer from this discussion for other forms of DIY biology and medicine? For one, the example of stimulant ab/use as it is happening and represented is a clear continuation of a historical trend: Not just the optimization of one's own chances in life and the desire to succeed but also the trend towards more patient participation and the staggering normalization of pharmaceutical drugs has empowered individuals to become active consumers instead of passive patients. As Chatterjee predicted, increasingly patients are the ones who request a certain type of treatment or who find loopholes in cases where their doctors do not cooperate with their wishes. The widespread use of Adderall and Ritalin for enhancement purposes by healthy individuals is a good exemplification of that, while 'brainhacking' or 'neurohacking' are continuations of the underlying desire into a sphere of unregulated, self-imposed experimentation. In a similar vein, it shows the example of an 'old' treatment now being used by people looking to optimize the self: Adderall and Ritalin have been used for decades as medical treatments, but their use today has taken on a new quality. This new quality in part is due to the increasingly blurry distinction between treatment 
and enhancement and the flexible boundaries of disease. However, this new use of an old 'technology' also shows how the use of a biotechnology decides about its social and cultural valuation and, vice versa, how social and cultural factors influence how biotechnologies are used. Especially the comparison to other, illicit drugs that belong the same class of controlled substances (such as Meth or Speed) shows how the evaluation of a drug is often a balancing act, a question of perception tied to the users and their context. Especially for other forms of 'commercialized' DIY, such as genetic or molecular direct-to-consumer testing - the topic of the next chapter - the lessons from this case study might be interesting. The overarching message of Take Your Pills is that while the ab/use of stimulants is nothing new, contemporary cultural influences are what is responsible for the current epidemic. Take your Pills rightly concludes that a cultural debate about performance enhancers as well as the underlying reasons for their use is needed, a debate that the documentary as pop-cultural artefact contributes to.

\section{Case Study IV: Make Your Own Medicine - The Example of the DIY EpiPencil}

In contrast to the previous case study, where the individual as consumer on their own pursuit of happiness and wellbeing was at the center of the DIY activities, this case study brings us closer to a maker-influenced definition of hacking and DIY. Though the consumer market does play a crucial role for the context of the DIY EpiPencil hack, the focus is on cheap, accessible and make-shift solutions. In 2016 the Four Thieves Vinegar collective revealed to the public their latest hack: A DIY solution to create an EpiPen, a staple item in households of allergy sufferers and one of the 'victims' of excessive price increases in the pharmaceutical industry. This hack brought them widespread news media coverage in 2016 and 2017. In this case study I will look at the Four Thieves Vinegar website, their introduction of the EpiPencil and the resulting media coverage to delineate how this endeavor was presented to and discussed in the public sphere. The self-description of the Four Thieves Vinegar collective's hack can be considered not just as an instance of self-marketing, but, as we will see, also a political statement, while the media coverage brought their efforts to a wider audience and thus contributed to the proliferation of 'pharmaceutical hacking' in the public imagination. Their 'hack' is presented and perceived as an intervention into a flawed system and demonstration that things could be done differently.

But first, let me go into a bit more detail on the individuals behind the hack: Michael Laufer, the chief spokesperson, and the Four Thieves Vinegar Collective. Four Thieves Vinegar could be considered a "DIY pharmaceutical company:" They operate around open access principles and individual choices, while their focus seems to be on distributive justice and access to medicine (Delfanti, Biohackers 38). The collective sees themselves as providing alternatives to the "disenfranchised" in the (global) medical market and as creating access to "tools" and knowledge needed to save lives. In their mission statement they state their goal as "Free Medicine for Everyone." This, for Four Thieves Vinegar, entails giving people tools to manufacture chemical drugs themselves everywhere around the globe, working against high prices for drugs, and thus giving all humans 
access to life-saving medications (Four Thieves Vinegar Collective, "Our Mission"). ${ }^{16}$ So while the DIY EpiPencil hack brought them the most media coverage in 2016, their main project is an Apothecary MicroLab that they claim could be used to manufacture small batches of medicines. Fittingly, the most prominent line on their homepage is "Make Your Own Medicine" (Four Thieves Vinegar Collective, "Home"). The focus of their MicroLab is on treatments for conditions like HIV, hepatitis, or toxoplasmosis and on reproductive freedom and abortion medications. Laufer presented the first prototype - including DIY manufactured drugs - at the biennial hacker conference Hackers on Planet Earth (HOPE) in New York City in 2016 (cf. Oberhaus). Four Thieves Vinegar, we can summarize, represents a hacker ethos, open access and open-source ideologies and DIY approaches so common in many DIY biology and medicine projects. The goal is self-sufficiency facilitated by access to knowledge and tools.

Fittingly, their hacks and DIY medical technologies are inspired by social debates and ethical considerations. The reason why their EpiPencil hit such a nerve and let to news outlets and magazines taking up the story at the time of its publication in 2016, was that they provided an answer to a widely discussed problem. In September of 2015 Martin Shkreli, hedge fund manager and CEO of various pharmaceutical companies, had publicly been heavily criticized for raising the price for Daraprim - a drug used to treat toxoplasmosis, amongst others in patients with HIV - from $\$ 13,50$ to $\$ 750$ per pill, sparking a public debate around rising prices for prescription medicine. ${ }^{17}$ Once a widespread debate about prices for medications was started, it is no surprise that also the price raise for EpiPens by producer Mylan in September 2016 sparked a public backlash. From 2009 to 2016, prices for brand-name EpiPens increased from $\$ 100$ to $\$ 600$ for a 2-pack of autoinjectors (Lyon). This considerable rise in prices for a single-use, lifesaving item did not only have many people question the integrity of 'Big Pharma' but also left some families dependent on epinephrine injectors without access. Even when their health insurance covered the costs, many families rely on multiple pens for easy access and safety. While generic injectors are available for less, 2016 was definitely a year of widespread debate about access to and costs for medications. This, of course, is partly due to the American insurance system where not all costs for medications might be covered or people might not have access to insurance, providing fertile ground for a form of medical segregation in which the well-off have access to medications and medical treatment while those further down on the social ladder do not. This is not just the

16 Thieves, criminals, outsiders, apparently, were also the inspiration for the name and the logo of the Four Thieves Vinegar Collective. Depicting a stylized plague-doctor or plague-mask, the story behind the logo comes from thieves who saved many lives through their ingenuity. As Piller describes it: "Instead, Four Thieves found inspiration and its logo from a bubonic plague episode in the Middle Ages. As Laufer tells the possibly apocryphal tale, thieves who made a habit of looting plagueridden areas protected themselves from infection by using masks containing vinegar and herbs with antimicrobial properties. Eventually, they were captured, then freed after agreeing to reveal their formula — which was made public, saving many lives. The story perfectly illustrates Laufer's model: 'an emancipation of knowledge."' (Piller and STAT) Laufer and the collective seem to see themselves in this tradition of saving lives, possibly also with illicit means.

17 Shkreli was later arrested for fraud in relation to his work for another fund, Retrophin LLC, in 2015 and found guilty in 2017, sentenced to seven years in prison and a monetary fine in 2018. 
general background and incentive for the projects of the Four Thieves Vinegar Collective, but it was also one the reason why the EpiPencil hack could reach a wider audience than just the 'typical' DIY hacker sphere.

The DIY EpiPencil, as the name already tells, is a self-made version of the expensive brand name epinephrine autoinjector built from off-the-shelf-parts and easily assembled for "just over 30 dollars." In short, it is built by combining an auto-injector meant for diabetics with a syringe and fairly large needle. While these three components are easily available online or in a pharmacy, the epinephrine that has to be manually drawn into the syringe has to either be sourced from a prescription or other creative sources. To complete the EpiPencil, Four Thieves Vinegar also provides a sticker with their Logo and instructions for its use. In a blogpost from Sept 29, 2016, the Four Thieves Vinegar Collective introduced the EpiPencil on their homepage. Apart from a short introduction to the parts needed for the EpiPencil, this introduction also resembles a political statement. They write:

WHEREAS The pharmaceutical industry continues to put profits above human life, and WHEREAS Autoinjectors and epinephrine are technology which belongs to the world, and WHEREAS EpiPens save lives every day, but only for those who can afford them, and SINCE The Four Thieves Vinegar Collective is dedicated to providing access to everyone WE HAVE developed the EpiPencil, an epinephrine autoinjector which can be built entirely using off-the-shelf parts, for just over $\$ 30$ US. Sources for the three items you require are linked below, followed by a video which details how to assemble them. Stay healthy, -The Four Thieves Vinegar Collective (Four Thieves Vinegar Collective, "Introducing", my italics)

Following their mission statement, the main motivation for the EpiPencil hack, they claim, came from their desire to give access to medical technology to everyone, not just those who can afford it. The overt critique of the pharmaceutical industry - their first point, you may note - is in line with their stance against profiteering from innovations and technologies by a few companies. This introduction positions the DIY EpiPencil not just as a creative, easy and cheap solution to a medical problem but also turns it into a social and political statement: DIY in the sense advocated by the Four Thieves Vinegar Collective is inherently political, an answer to the contested issues of its time.

The access they promise, then, is promptly given: A list of links guides the DIYer to the right sources for the required components and a video gives them a practical manual on how to put the parts together safely. Seemingly shot with a webcam, this 5.48 min video features Dr. Michael Laufer in a home-like environment: Laufer sits on a chair facing the camera and demonstrates how to assemble the DIY EpiPencil. People, he opens the video, have asked them to do something about the EpiPen scandal and Four Thieves has delivered. What follows is a brief tutorial, that apart from some fiddling with the needle to make it fit into the autoinjector properly can be easily followed in minutes. The result, Laufer says, is an epinephrine autoinjector that "functions just like an EpiPen" and can be used "with no special training" (Four Thieves Vinegar Collective, Epi Pencil). The aesthetics of the video underlines the DIY, home-made, workaround approach proposed by the hack. Instead of producing a high-quality one, this video is focused on what it tries to carry across: how easy and fast you can assemble 
a potentially life-saving medical device. To highlight the functionality of the EpiPencil, Laufer includes a scene in which he uses it to inject himself with saline solution, representing the self-experimentation ethic so common in biohacking. Roughly a year later, in 2017, the media coverage of their hack prompted the Four Thieves Vinegar Collective to publish another video update for the EpiPencil that contains answers to the most frequently asked questions. It goes into detail on sources for epinephrine other than a prescription, the EpiPencil shelf-life, sterility, syringes, dosage for children, the lack of options as substitutes for epinephrine, other brands and generic injectors as cheaper alternatives to the brand-name EpiPen, as well as the legality, reliability and regulation of (DIY) medical devices (Four Thieves Vinegar Collective, Epi Pencil Update).

The DIY approach espoused by the DIY EpiPencil follows the sharing and hacking ethos so fundamental to the maker movement as well as the focus on individual choices proposed by personalized medicine: Adhering to an open source ideology, plans and instructions for the EpiPencil and the MicroLab can be freely downloaded or accessed by everyone; the solutions they propose are, as their name already implies, the result of collective and collaborative work; their political messages show a marked distrust of pharmaceutical institutions; and their hacks are innovative and creative combinations of current technologies and make-shift solutions. This hacker approach is also part of the press coverage of the EpiPencil. The media representations under consideration here - five articles published between September 2016 and July 2018 - all include some form of reference to the biohacker or DIY bio-movement into their discussion. In the earliest one Jamie Condliffe, for the MIT Review, describes Laufer and Four Thieves Vinegar as "DIY enthusiast" and "biohackers," who build "home-brew" versions of medical devices (n.p.). Interestingly, this word choice does not only position them in the biohacker and domesticated biology movement but also close to the famous hacker, hobbyist and DIY computer club, the 'Home-Brew Computer Club' in Silicon Valley (1975-1986). In an article for Vice-daughter Motherboard, journalist Daniel Oberhaus describes the workarounds and creative solutions that the collective has found for the manufacturing of their drugs. "T[t]hese sorts of unorthodox approaches to healthcare," he writes, "are the name of the game in pharma hacking, where the goal is to help people at any cost" (n.p.). As is the goal of DIY biology, the result of such "unorthodox approaches" are cheaper, easy to use solutions. The hacker ethos visible in the DIY EpiPencil makes it a good example of science and medicine, or here pharmacology, as a bottom-up process. ${ }^{18}$ Of course, such approaches 'from below' also generate a lot of controversy exactly because they do not come from professionals or institutions. In the case of medicine and pharmaceuticals, such approaches become even more disputed because they are not concerned with harmlessly 'playing' or 'tinkering' with biological materials but moving into the sphere of actual cures - DIY copies of commercial medications used to treat the sick - where questions of regulation, safety, testing, errors and potential harm are particularly important.

18 Another example is the Open Insulin Project, a Bay Area biohacker team that works on creating a new protocol for insulin production, undermining intellectual property to create "newer, simpler, less expensive ways to make insulin." Their aim is to make insulin freely accessible for everyone by creating an open protocol for its production, cf. Open Insulin Project. 
In 2017, Scientific American ran one of the more in-depth stories on Laufer and the DIY EpiPencil, in which the author Charles Piller describes Laufer as a "fixture in the growing biohacker movement" (Piller and STAT, n.p.). But more than that, this article seems to have set the stage for one of the most notorious labels used for the Four Thieves Vinegar Collective to date: Anarchists. "An Anarchist," according to their title, is "teaching patients to make their own medications" with the goal of building "a DIY movement to undercut high drug prices," as the subheading suggests (n.p.). This label is taken up by Daniel Oberhaus in 2018, when he invites the reader to "Meet the Anarchists Making Their Own Medicine" (heading) and describes the Four Thieves Vinegar Collective as "a network of tech-fueled anarchists taking on Big Pharma with DIY medicines" (n.p.). Sanctioned by Laufer himself, this label tells us a lot about both his own and the public's perception: Rarely valued positively, anarchists are known for standing against an established order and rebelling against authority - hacker ideals par excellence. Anarchism can mean both a chance at fundamental change in an unjust system and a descent into chaos. It is this ambivalence that Laufer and the media seem to play with. On the first glance negative, they are nevertheless also branded as a disruptive force working against the system to achieve higher moral ideals. In Daniel Oberhaus' report for Motherboard/Vice from 2018 the anarchism is acknowledged as part of the Collective's agenda as well: Outsiders stir up a market dominated by expertism, elitism and corporations. In fact, rebelling against the system, in Oberhaus' article, seems to be a good, praiseworthy quality: "By freely distributing plans for medical devices and pharmaceuticals, a loose collective of anarchists and hackers is threatening to pull the rug out from under one of the most regulated and profitable industries in the world. And they're just getting started" (n.p.). The narrative Oberhaus creates reads more like an epic 'David and Goliath' tale where the underdogs fight for the good cause.

This preoccupation with anarchy demonstrates that here hacking to a certain degree also takes on some its illegal, shady connotations: The devices and technologies proposed by Four Thieves Vinegar are moving in the grey zones of the law, are unregulated and unruly, positioned against the authority of government bodies and pharmaceutical companies. In their update video (2017), Laufer goes into detail about the regulation of medical devices, saying that ideally the infrastructure is such that you do not need to revert to DIY solutions, but in the current situation, in the end "[i]t is a personal choice, and that is where we think the choice should lie, with the individual so they can manage their own health" (Four Thieves Vinegar Collective, Epi Pencil Update). Not just for Laufer and the Collective but also for some media representations the central question is the freedom to choose. D'Adesky, for example, claims that this question is at the heart not just of many debates in medicine but also of the right to use DIY EpiPencils: "Should we be free, as individuals, to make and take our own medicine at home? Who's responsible if we get hurt or die? Do we have to [sic!] right to do what we wish with our bodies in the interests of survival, healing and self-care?" (n.p.) That she raises these questions points to central pillars of American cultural identity: individualism, autonomy, self-sufficiency. Laufer would answer all the questions of above in the clear affirmative. Oberhaus takes up the idea of autonomy: "For Laufer, Four Thieves is as much about medicine as it is about the right to the free flow of information and personal autonomy. As far as he's concerned, one cannot exist without the other" (n.p.) 
This right to information and knowledge, the question of access, for Laufer is intimately tied to the right to use that information. In fact, Oberhaus ends the article on a quote by Laufer: "Pursuing science is a human right,' Laufer said. 'In fact, it's the human right from which all other rights flow. You have to be able to do whatever you want to your body and to think the way you want"' (n.p.) Here, Laufer argues not just for open access to scientific knowledge but also for complete autonomy over an individual's own body as a human right.

Complete autonomy, however, also raises questions of regulation and safety: Should individuals be allowed to use such hacks, even if they might harm themselves? Or should they be regulated or prohibited? "Home Pharma Hacks Raise Safety Questions" writes D'Adesky. In her report doing it at home or in your kitchen is automatically assumed to be more dangerous because of the lack of standardization and quality checks (n.p.). Critics, she writes, believe hacks are dangerous and pose safety risks exactly because they are not tested. But she also acknowledges that these safety concerns are on Laufer's and the Collective's radar: While controls for drugs and devices marketed to consumers are deemed necessary also by Laufer, security concerns for him are only a "first-order reaction" that ideologically will always come behind "more access to more information" (D’Adesky, n.p.). Similarly, Oberhaus includes voices and opinions of MIT researchers to underline the fact that safeguards and quality controls are needed to make pharmaceuticals and their DIY equivalents safe for patients. Four Thieves, he writes, "walks a fine line when it comes to the legality of their enterprise" (n.p.). But, again, he acknowledges that they are conscious of the pitfalls as well as the chances. They are, he says, not "naive" about the risks. Because they realize that instructions can be followed incorrectly - with possibly toxic or deadly results - they are actively working on reducing "the likelihood of this happening:" one of their "most significant contributions to DIY medicine" he writes "is prioritizing harm reduction in its research and development" (Oberhaus).

These basic ideals of American culture - choice, autonomy, self-reliance - that are reflected in the DIY EpiPencil hack and its media representation point to their deep embeddedness in their time and place. Oberhaus, for example, tries to expose the many cultural and social factors that contribute to the appeal of hacked solutions and DIY approaches. The hacks are portrayed as reactions to high costs and shortage of EpiPens or the scrupulous raises in prices for pharmaceuticals by Big Pharma (Oberhaus). Cultural factors, as well as the political situation, are shown as responsible for why people not just decide to join the collective but also why their solutions have a certain appeal also for critics. In an insurance and medical market like the US even the sharpest critics cannot "dismiss Laufer outright" (Piller, n.p.). In the article, Dr. Vinay Prasad argues that Laufer is a "symptom" of the disease of drug pricing, a "symptom" and reaction to larger, as of now unsolved, issues. Laufer, even a critical reporter like Piller has to acknowledge, has a point to what he does, he can work as a conversation starter for larger public debates.

In the media accounts under consideration here, the DIY projects and activities proposed by the Four Thieves Vinegar Collective, are framed as a form of creative intervention into problematic systems of power and dependence. Condliffe for the MIT Review, for example, advices caution but sees hacking as a valid way to prove a concept and/or show 
alternatives. In this case, the EpiPencil is a persuasive demonstration that it is possible to manufacture much cheaper devices, which confirms the industries' profiteering (n.p.). Even though Oberhaus' article also cautions about safety issues, he nonetheless also describes Laufer's activities as "a valuable form of social activism that points the way to a promising future" (n.p.). Likewise, Josiah Zayner, known biohacker and CEO of The Odin, interviewed by Piller for his article, claims that Laufer's work is a "symbolic force" (n.p.) The DIY EpiPencil becomes a symbolic act, an example that you can do it otherwise. It is, as also Four Thieves Vinegar acknowledges, not an approach suitable for everyone, or one that should be necessary, but a (political) demonstration meant to address a pressing issue as much as a project for more adventurous hackers and tinkerers. The media representations seem to value that such an intervention - even if never needed or used - highlights the point of the debate: the actual costs for parts and production versus the price asked by the pharmaceutical companies. Creative hacks for them can be used as illustrations that contribute to a public debate. By doing so, they also validate the political impact of the hacker ethos and DIY approaches as a form of protest and creative intervention. In fact, as Matthew Donovan writes in his analysis of the biohacker activism in resistance to the 2016 EpiPen price crisis, Four Thieves Vinegar has inspired other makers and hackers to create alternative solutions to this problem as well. Even if they will never be used by the masses, their existence and the debate around their use, ethics or value is starting conversations, in this case around how health care should be structured, who should have access and at what cost. Against the social and political backdrop of their development, hacks such as the DIY EpiPencil become instances of "critical making" (Richterich 160). Oberhaus claims that Laufer has "turned subversion of the medical industry into an artform" (n.p.). Maybe this statement, here, should be taken more literally: The EpiPencil and the MicroLab are then seen as examples of the ingenuity and creativity of the hacker-maker-DIY movement, while also, and crucially, retaining their political power.

\section{To Summarize}

What then, the attentive reader might ask, does this form of DIY as creative, political intervention have in common with the commercialized use of stimulants for self-enhancement? Both topics, of course, go into completely different directions: What connects them superficially is their focus on individual choice (and all the consequences that could entail) and questions of access to and regulation of medications and knowledge. But, on a broader scale, the much more important connection is their foundation in highly related social and cultural issues: In case study III, the culture of constant competition, performance and success in neoliberal capitalism invites (cognitive) enhancements to 'get an edge' over the competition. But more than that, as Take Your Pills also thematizes, the use of these types of drugs is also connected to socioeconomic factors: Individuals do not only need money to afford such types of self-enhancement but also access to and the ability to negotiate the health care sector. Both, according to Chatterjee in the documentary, are a matter of privilege (31:05). The "disenfranchised," in Laufer's words, are not part of this equation. In case study IV, structural factors in the U.S. health care and insurance system but also globally in the unequal distribution 
of resources and access are what makes 'alternative,' self-made options necessary. DIY, here, becomes not a matter of privilege but a solution. Both practices thus in one way or the other also address some of the most pressing cultural dilemmas of our time. DIY, in both studies, provides a form of self-empowerment: on the one hand, to gain access and potentially save a life, on the other, to better allow individuals to cope with and navigate a potentially structurally flawed system of global capitalism. Could, in a potential future, the DIY technologies proposed by Laufer not just be used to grant access to medications but also to allow people to manufacture their own enhancement cocktails? Such questions do not only highlight the urgent need for public discussions about what types of societies we as humans want to live in, but they also illustrate once more that treatment and enhancement are often just a question of use - and those uses need to be discussed as well. 



\section{Test Yourself - Making the Invisible Visible}

As Henk ten Have predicted in 2001, this millennium, post-Human Genome Project, is indeed characterized by a widespread availability of predictive knowledge and genetic and molecular information about the self (301). The Human Genome Project had not just popularized genetics for a wider audience - thus changing fundamentally how humans view themselves - but also lead to a cascade of technological innovation that significantly reduced the costs and the time needed to perform genome analysis. Since the first decade of the century, more and more complicated genetic tests are performed and marketed routinely, to physicians but also to consumers themselves (cf. Hamburg and Collins 303). Even though they are probably the most prominent example, tests available to the consumer today are not just limited to genetics but also includes 'molecular' testing in the wider sense: Vitamin deficiencies, hormonal status, infections, gut health, all these can be tested from the convenience of your home. They do not need a physician as intermediary but rely on individuals doing the first steps of the test the needle prick or specimen collection - themselves. You no longer have to consult a medical professional to have blood work ordered, you can do it yourself or, rather, have a company do it for you. The possibilities range from home health tests to companies that offer all-inclusive packages, including recommendations on how to make the best use of your results. ${ }^{1}$ They are part of the aim to personalize medicine, to tailor medical interventions to the patient, thus reducing costs and improving outcomes. For some critics, these types of tests can also be considered the "epitome of a particularly individualist or consumerist approach to healthcare" (Turrini and Prainsack 4). Their foundation, thus, lies also in the consumerist ideology of the DIY patient.

As Turrini and Prainsack summarize from different, mostly empirical studies, users and the general public tend to evaluate contemporary (genetic) testing options positively because of their presumed medical importance - presumed in this case because that is how they are marketed to the public - and the potential for positive changes towards a

For example, Amazon.com offers around 30,000 matches for the search term: "Health Tests." These range from DNA tests to $\mathrm{pH}$-level tests or sperm count tests for your iPhone, but also include more mundane tests such as blood glucose or iron deficiency (search done: 04.09.2018). 
healthier lifestyle (5). ${ }^{2}$ This presumed value is one of the reasons why DTC testing is so popular today. "Genetic testing is capturing the attention, and imagination, of people," write Anna Harris et al., who not only find their (popular) media littered with molecular breakthroughs and personal experiences with direct-to-consumer (DTC) tests but can now buy these tests conveniently from a multiplicity of sources via the internet, the primary medium through which they are marketed, sold and shared (60-61). The internet has become a convenient tool to sell tests, built communities of users and popularize their use. However, this growing industry of pre-symptomatic testing and molecular diagnosis is also part of the promissory culture of biotech intervention (Rose, Politics of Life 91). Press releases, marketing materials, and public appearances of testing companies promise to provide the consumer with some of the most-thought-after goods of today: (self-)knowledge through science, potential for optimization, confirmations of one's uniqueness and individuality but also connection and participation in something larger - common concerns that my analysis has revealed.

While some critics rightly celebrate DTC medical testing as a form of patient empowerment, others focus on the negative side effects of this type of empowerment: the risks associated with giving consumers access to highly predictive and uncertain information about their own bodies without the guidance of medical professionals; the new types of responsibility and obligations the patient is ascribed; the uncertainty of the testing procedure itself and the possible impact on the individual; the effects of heightened individualism on social solidarity and society as a whole. The discourses of empowerment are always in stark contrast not just to the new burden of individual and collective responsibility for personal health but also to the new forms of precarity and vulnerability that these types of predictive knowledges about the body can entail. ${ }^{3}$ These concerns have sparked theoretical and ethical debates, giving rise to new labels for the tested person, such as the "potential sick role" (Lupton, Medicine 98-99), the "person at risk" or "susceptible self" (Rose, "Genomic Susceptibility" 147). Such delineations point to the main concern: the individual is placed in a new environment of risks and possibilities, for which they often lack the biological literacy to correctly interpret them themselves, potentially leading to anxiety about the contingency of the body. This feeling of vulnerability to forces beyond one's control inverts the aim of empowerment and instead promotes new forms of self-government to avoid said vulnerability.

The cultural discourse created is that more knowledge about the inner workings of the body allows us to understand ourselves in new ways but also to optimize those inner workings: Knowledge leads to intervention, to change, to manipulations of those biochemical pathways that were found deficient or lacking. The belief is that new medical technologies can "make the invisible seed of future health or illness visible" (Rose, "Race, Risk" 432). Biomedical tests, thus, are perceived to, in Chrysanthou's words, give us the

In contrast, they also show that evidence for health-related behavior changes cannot be found in many of the studies done with DTC testers (Turrini and Prainsack 5).

These vulnerabilities can arise either through the uncertainty of tests results themselves (cf. Stevens 203, 292) - as predictive susceptibilities or numerical assessments of risks, sometimes even based on shaky correlations; the in ability to act or intervene (e.g., in untreatable diseases); or through negative psychological reactions (cf. Turrini and Prainsack 4). 
"power to render the invisible visible" (475). "Haunted by uncertainty," the utopian body, for him, needs access to "its own personalized map of its interior landscape" (Chrysanthou 476). The tests, it is believed, can make the material basis of life - with its flaws and individual characteristics - visible, actionable, create a "map" of its interior that the individual can use to reach its goals of fitness, health, or self-knowledge. But the "invisible" here are not just material structures but also possible futures, possible identities.

Testing, as this introduction meant to show, can have a variety of material, psychological, even social effects. When tests are done individually, without the interference of medical professionals, self-prescribed and self-administered, they become a form of DIY biology, a sometimes playful, sometimes serious engagement with one's own body and the biological building blocks of life. Turning bedrooms, kitchens, and couches into makeshift laboratories, direct-to-consumer testing is the subject of case study V "Welcome to You." Empirical research about the testing process, the motivations to participate and its psychological and physical outcomes has been underway for quite some time. ${ }^{4}$ What interests me here is not so much the perspective of the user but how the companies represent (and thus market) their tests to the public, how they create promissory discourses of self-knowledge and public participation. I will therefore primarily analyze the individual company's websites and bring them into conversation with each other. This type of representation draws on common ideologies and desires of the public - it identifies a need and offers a solution. It thus reflects some of the affective structures behind (commercialized) DIY biology. ${ }^{5}$ Due to their desired audience and purpose as a marketing tool, it is fairly easy to find material for rhetorical and contextual analysis and similarities in the discourses they create: discover yourself, improve your quality of life, enhance your performance, get to know yourself with science. However, in this process of self-discovery age old techniques such as introspection and verbalization are replaced by proactive preemption-prevention based on consumerist ideologies. Even though on it is a highly individualized action, direct-toconsumer testing is discursively aligned with DIY biology and citizen science. In the second case study, then, I turn from those tests that rely on laboratories for analysis towards examples in which the testing process is 'do-it-yourself' from start to finish, 'hacked' and open solutions to biomedical testing. Case study VI, "Make it Personal," is mainly concerned with the cultural representation of a specific case - Kay Aull's DIY hemochromatosis test - in Wohlsen's eponymous non-fiction account of Biopunk, interspersed with other examples of how biomedical testing can be realized with a more explicit DIY approach. What both case studies have in common however is the idea that 'testing' the molecular make-up of the body will lead to new insights into the self, new forms of (social) connection and possibly avenues for self-enhancement. utility of DTC tests.

5 It thus, to a degree, also connects back to the question of DTC advertising and its inherent positive and negative sides, a factor that also turned the stimulants described in the previous chapter into such a cultural commodity. 


\section{Case Study V: "Welcome to You" - Direct-to-Consumer Testing and Citizen Science}

With the "explosion" of genetic testing companies, "the intersection between genetics and medicine is starting to be tapped by amateur biologists-at-large as well," writes Elie Dolgin (953). However, not just genetics is increasingly accessible for DIY biologists but also molecular biology more generally. Probably due to the higher risks and privacy issues connected with the 'data' they reveal, genetic tests have received the most widespread attention. Molecular and biochemical DTC tests, however, are no less telling than their famous cousins, especially because the discourses they create are often similar: They all speak to an appeal of self-obtained information, of active participation and curiosity about one's own biological makeup. Despite debates about their efficiency, accuracy, and safety, the need for professional counseling in the process, or the lack of literacy on the part of those who opt for the tests, their popularity seems to be unbroken, visible in the hundreds of companies that offer DTC test in one way or the other. As I have argued previously, genetics might be the most widely mentioned example of the molecularization of the human body, but it is by far the only one. The 'inside' view, the fragmentation of bodily functions and their embedding into molecular and biochemical pathways, is not limited to the genome. Rather, the availability of testing tools for DIY users - from home tests bought on Amazon to collection kits analyzed in a lab - speaks to the increasing desire of individuals to gain a deeper insight into and understanding of their bodies on this molecular level. They want to translate the prevalent molecularization in cultural discourses of the body onto a more personal, individualized level and make it 'actionable' for their own wellbeing.

The DTC testing industry has been following the same broad premises ever since its inception: Consumers choose and order a kit online, collect their own biological specimen - from stool, to saliva, to blood, the primary difference being the material they work with - and send it to a company for analysis. A few days or weeks later, they receive their results in their (virtual) mailbox. The genetic and molecular testing companies in this case study follow this pattern and range from DNA tests for ancestry and health, to blood tests as well as 'wellness' tests on a variety of biological specimen. ${ }^{6}$ Before I go into a more detailed analysis of the semantic characteristics and overall themes, let me provide a bit of general information about the companies and their products one-by-one.

$23 a n d M e$, of course, is probably one of the best-known providers of personal genomic services in the US and world-wide. Founded in 2006, their tests provide the customer with information on their individual genetic variants - mostly on SNP-level - with respect to their ancestry, their genetic health risks and carrier status, as well

6 Another highly interesting example is DIY testing for sexually transmitted diseases and reproductive health: myLAB Box ${ }^{T M}$ is a company that promises its users discrete and accurate testing for some of the most common STD/STIs, such as Syphilis, HIV, Chlamydia, or yeast infections. They position themselves as a means to take control of your health from the "comfort of your home" $\left(\right.$ myLAB Box $\left.{ }^{\top M}\right)$. What makes it so interesting is the stigma - and thus immense hurdle to seek treatment or get tested-around STDs/STIs. 
as their wellness predispositions and individual traits. ${ }^{7}$ Since 2010 uncertainties about their status as medical devices, their FDA approval, and the potential for unverified information and false results had let to debates about the regulation of $23 \mathrm{andMe}$ and similar companies' tests, leading in November 2013 to FDA warning letters to suspend testing. 23andMe complied and suspended sales for the medical component of their tests, sought FDA approval and now is the first company whose DTC genetic tests were granted FDA authorization in 2015. But more than providing individual information, 23andMe also invites their users to share and compare their genetic results, find genetic relatives through their platform or participate in genetic research based on their data and health information, which adds a social, collective dimension to their testing (23andMe, "DNA Genetic Testing").

InsideTracker is a tool that also Meisel recommends in his guide to biohacking: The company focuses on molecular testing, more specifically they provide bloodwork for their clients and turn the results into "actionable recommendations" to optimize health, wellness and performance. They offer a variety of "plans" in which up to 41 "biomarkers" are analyzed. As part of their marketing strategy, they recommend regular testing to compare results and see which of the changes or recommendations work on the molecular level. By "tracking data" over time, the goal seems to be to create a continuous picture of the "inside," the molecular make-up of the body (Segterra/InsideTracker). While InsideTracker seems to endorse physical activity and high performance, Wellnicity, as the name already suggests, looks at a more abstract notion of "wellness," which in their offers "meets simplicity." They do not only provide molecular testing but also offer to put together a personalized "supplement regime" of "professional-grade Vitamins." In a promotional video that explains the process of testing, Wellnicity positions itself as a means to uncover the connection between different symptoms one might experience to one's overall wellness. On their webpage, users can either browse the collection of tests and testing packages they offer or select a kit based on individual health goals, symptoms or concerns. The tests themselves, then, range from brain health, gut health, testosterone, stress, metabolism, thyroid, food sensitivities, Vitamin D, to blood sugar levels. Interestingly, many of the them are named along the same line: "My Stress," "My Metabolism," "My Gut-Health," promoting their "personal" nature to the consumer (Wellnicity, "Wellnicity"). The benefits presented by the companies - self-knowledge, the possibility for early intervention, empowerment - as well as potential problems and concerns - uncertainty, results that are not actionable or misleading, lack of treatment options, unintelligible results, lack of literacy, undue responsibilization - are strikingly similar among them.

As for all the genetic testing companies, the analysis of DNA samples is mostly based on SNPs. An individual's profile is created to compare variations at single base pairs at known locations where variations often occur. Based on Cenome Wide Association Studies (CWAS) and the correlations they found between SNPs and diseases or traits, an individual's disease risks are calculated. When people access the results online, they are provided with information on the location of the variation and their individual probabilities. In recent years, companies like 23andMe have established measures that allow individuals to choose which information they want to see, especially for diseases like breast cancer, Alzheimer's or Parkinson, where the correlation is relatively certain but treatment options radical or practically nonexistent. 
In these tests, "[b]iology is not only mutable, changeable, and manipulable - it is also utterly consumable," one of the most valuable commodities (Levina 5). What is paid for by the consumer are knowledge and access to their own (genetic) information. DTC tests represent one of the most prominent examples of a downright assimilation of DIY ethics into a consumerist ideology. The focus on knowledge and access is also visible in the rhetoric created by the company websites: Access to knowledge is a form of empowerment necessary for individuals striving to feel their best, as Wellnicity promotes (Wellnicity, "About Us"). Possessing knowledge and information, then, is positioned as enabling people to be "in control" of their body and genetic information. This idea of control is often connected to the notion of "taking action:" Most overtly, InsideTracker urges their users to "take action using your blood, your DNA, your habits:" Instead of "guessing" - with its connotation of uncertainty - people should "know" what their bodies need (Segterra/InsideTracker). Information leads to control leads to action, the dominant logic goes. The encouragement to "take action" by all these companies can be read as form of responsibilization for one's own wellbeing: it is the individual that is responsible for using their new knowledge to actively adapt their situation.

This combination of information, action and responsibility in the name of empowerment is visible also in the narratives that 23andMe uses as customer testimonials. By chance, "Jill" discovered a BRCA-1 mutation in her test report that puts her at higher risk of developing breast cancer. After the test and a clinical risk assessment through her doctors, she opted for a preemptive double mastectomy. In her video she talks about how the experience of using her genetic information to do something about her health was very empowering: "Why wouldn't you want to know? Why wouldn't you want to do something?" (23andMe, "Customer Stories") For her, that knowledge allowed her to make an active decision about her health and (future) wellbeing. That one would react to the information given - even go to drastic lengths to prevent that risk from coming true - seems to be self-explanatory. ${ }^{8}$ DNA information here becomes transformative, even performative for an individual, in the sense that it starts many processes of treatment or changes in lifestyle that may affect their sense of self as a person-at-risk for example or a prudent biological citizen. ${ }^{9}$ The discourses of empowerment and the resulting promotion of the ideology of self-responsibility explicitly or implicitly created by many of the websites promotes the idea and necessity of proactive preemption-prevention. It persuades individuals to actively seek out information about themselves, empower themselves, to then act responsibly in relation to that information by changing their habits and lifestyles. Two prominent problems arise here: First, the information you get is not necessarily actionable, thus turning the accessed knowledge into fun trivia 
without long-reaching effects on health or wellness. This is one of the reasons why companies like Wellnicity and InsideTracker offer to make it actionable for you. The second problem is one of differentiation: This type of commercialized access might lead to a stratification into those who can afford to test and those who cannot, with consequences for the self and society as a whole.

The idea of taking action is not just integrated in discourses of health and healing (or prevention of disease) but also much more generally in ideas of self-enhancement. As some of the testing companies propagate it, the aim is to optimize one's own wellbeing and performance. As such, some of the tests can be counted as examples of the much more literal "makeover culture" of beauty (Wegenstein, Cosmetic Gaze 111). ${ }^{10}$ They are one of the ways in which we are bombarded with messages of potential optimization of different facets of our lives. In essence, the idea of optimization perpetuated here goes back to the same premises of competition, getting an edge, performing better than others, and maximizing life satisfaction through investment in bodily capital and social status, that I have discussed in relation to self-help guides and pharmaceutical optimization. The molecular tests performed by InsideTracker are an especially interesting case: Already their different tests exhibit a downright adoption of (neo-liberal) goals of self-management and self-optimization in the name of the market: They have telling names such as "Ultimate" (tag line: "Take control of your destiny"),"Vitality" ("Get the competitive edge"), "High Performance" ("Push your performance past its peak"), "Essentials" ("Improve your body and Mind"), or "InnerAge" ("Gain Life and Vitality") (Segterra/InsideTracker). The discourse created only by those names indicates the main goal of the tests: improving health and performance, reaching goals through individualized action, and a sound foundation in science. ${ }^{11}$

If we believe the promises made by the web appearances of these DTC testing companies, however, genetic or molecular knowledge can not only help you to optimize the self but also to discover yourself. The idea is that you can discover the 'true you,' your essence, through your biology, thereby positioning the self as somehow fixed in the body, determined by its materiality. DTC testing, writes Stevens, delivers on the promise of the Human Genome Project to tell us something about who we are (Stevens 307). Biological 'data' are positioned as a repository of information about the self. The discourse of personalization and individualization is extremely prominent on many of the webpages: Wellnicity and InsideTracker advertise their "personalized," "individualized" or "customized" solutions. On their saliva collection kit, 23andMe writes: "Welcome to you." (23andMe, "DNA Genetic Testing"). ${ }^{12}$ An abstract "you" is the primary addressee

10 Some companies like the internationally operating DNAfit take this connection one step further, when they portray genetic information as a source to optimize personal fitness and body shapes by providing information on optimal diets, nutrient needs, exercise responses or recovery profiles (DNAFit Life Sciences Limited/Prenetics).

11 A science-based approach is another joint keyword for many of the companies: Authorization for the tests and the companies comes from industry leaders such as biotech companies or universities, government oversight bodies such as the FDA, industry standards and the culturally entrenched trust in scientific solutions.

12 An individual, for them, is a system of cells that awaits to be explored: "You are made of cells. And the cells in your body have 23 pairs of chromosomes. Your chromosomes are made of DNA, which 
of the websites - translated by the reader or user into a very concrete and personal "me." InsideTracker uses a related and highly interesting image: "A selfie from the inside. It's customizable. It's simple. It's based on you." (Segterra/InsideTracker, my italics) A primary mode of representing yourself, the selfie has become a pop-cultural icon today. ${ }^{13}$ Here, we find a molecular "selfie," a molecular representation of the self, of "you" - visible and abstract at the same time, a collection of data points. 'Yourself' is thus positioned rhetorically at the center of the testing process. The 'you' becomes a stand in, a possibility - 'That could be me!' - an affective appeal. Surrounded by images of made-over bodies, claims Wegenstein, we begin to desire a make-over ourselves (Cosmetic Gaze, 2). Surrounded by molecularized, mediatized bodies, we begin to desire our own molecularization and the possibilities it might entail. But this 'you' is also a celebration of uniqueness: "23 pairs of chromosomes. One unique you." (23andMe, "DNA Genetic Testing"), "No two bodies are the same." (Segterra/InsideTracker), "Wellness is...a personal journey unique to each individual." (Wellnicity, "Wellnicity"). In DTC testing, it is implied, the focus is on you, your individuality, your personal "uniqueness." Consuming apparently individualized solutions becomes a means to ascertain one's own individuality and uniqueness in a time of increasing uncertainty and loss of traditional anchors of identity.

Nordgren and Juengst argue that the webpages of genetic testing companies link genetic and personal identity by adopting the rhetoric of "personalized medicine," which suggests that the information they provide is uniquely tailored for an individual or will provide new (fundamental) insights into their identities, in relation to themselves and their family lineage or group membership. In this way, they claim, in the discourses created by the testing companies "the genome can perform all the philosophical functions of the soul in providing an ontological basis for our unique identities" (159-60). This appeal to the genome as a secular substitute of the "soul" is one of the reasons why such companies are so appealing to consumers: Nordgren and Juengst claim that their business models draw together three different ideological streams in contemporary EuroAmerican culture: "the distinctly pre-modern search for a naturalistic understanding of individual identity in a pluralistic world, the thoroughly modern cachet of genomics as a science, and the post-modern emphasis on radical individual self-determination" (15758). These three ideologies - the search for a naturalistic identity based on biology, the belief in science, and the emphasis on self-determination - flow together in 23andMe's campaign "this is me ${ }^{\circledR ”}$ that they use for their "customer stories" and "genetic journeys." Grounding the self firmly in its biology, such a genetic statement of also implies a longforgone form of genetic essentialism (cf. Nordgren and Juengst 157-58). But it is also a form of self-declaration, a form of storytelling about the self based on its molecular make-up.

can tell you a lot about you. Explore your 23 pairs today." Apart from this apparent molecularization of the body,23andMe positions their tests as "easy ways to discover you" (23andMe, DNA Genetic Testing) - finding the self in its molecules.

13 We could argue that this analogy to a selfie - a medium prone to sharing - also buys into a similar ideology: The molecular selfie, as much as the visual one, is meant to be shared, liked and commented on by others - thus providing another example of the collective nature of individualized testing. 
Turrini and Prainsack see identity making as one of the possible uses and values of DTC tests: DTC testing becomes a means of "exhibiting and speaking about" one's genetic self, it serves as a "starting point to tell a story about ourselves." As such, DTC testing is also "inherently relational:" "when genomic data are used to explore or reiterate one's identity, these activities simultaneously build, change, and reinforce relations to others" (Turrini and Prainsack 5-6). It is not just a story about the self that is told but also one about those close to the self, living and dead, even society as a whole. ${ }^{14}$ This is visible in DTC testing for health, as the results often concern also current and future family members, but even more so in testing for ancestry. DNA testing is positioned as a way to look into the past and uncover all those connections to other people on the globe - an individual's group connections and tribal belongings. ${ }^{15}$ 23andMe also encourages their clients to test several individuals from one family for comparison and to search for relatives through their website: A "DNA-based versions of social networking in which connections can be made on the basis of matching genotypes," claims Stevens (304). On their webpage, 23andMe advertises for their ancestry testing not just with the possibility of experiencing one's ancestry in a new way - gain new genetic knowledge - but also by promising possible connections with "DNA relatives" (23andMe, "DNA Genetic Testing"). ${ }^{16}$ In this way, genomic research becomes, in Levina's words, a "social-networking" activity (4). This ethos of sharing, one can argue, has also taken inspiration from the sharing culture propagated by social networks like Facebook and Twitter (cf. Levina 4) and perfectly aligns with some of the values of the maker movement and DIY biology. According to Stevens, linking genomics with the internet and social media - through its digitization, and I would argue, the discourses created by genetic testing companies "encourages" individuals to think of their genomes as similar to those types of information about the self that are today routinely shared online (307-08). This positioning

14 Genetic genealogy is a highly debated issue that has received more and more media coverage in the past years: Some public DNA databases on which people have uploaded their DNA for genealogical inquiries, have allowed law enforcement agencies to use their databases to solve unsolved criminal cases. From DNA hits in the databases genealogists can infer whole family trees and identify possible subjects based on their DNA, a prominent example was the capture of the "Golden State Killer" in 2019, almost 30 years after his crimes ( $\mathrm{H}$. Murphy). A big issue that arises is that of informed consent: If one member of a family uploads their DNA profile, parts of their relative's DNA is revealed as well - possibly without their consent.

15 Not only is genomic testing for ancestry not an exact science (cf. Balding et al.), it also has possible racial implications. In Private Bodies/Public Texts (2011) Karla F. Holloway criticizes how despite the claims that race is not discoverable, it does "inform" genomic science (79): Social assumptions, for example, are carried from (racialized) language to the data, scientists often have to make ,arbitrary choices" about designations and migratory patterns (81). Seemingly "benign" ancestry testing contributes to the growth of DNA databases, whose private and public uses, for now, remain opaque (88-89). We do not know how companies will use these databases in the future, if privacy and confidentiality are observed, or whether they will be misused, for example by law enforcement agencies, as described above. Against the backdrop of prevailing stereotypes, assumptions and racial discrimination such misuses could disproportionately affect African-Americans, for example in race-based DNA sweeps (Holloway).

16 Similar discourses are created by other companies that provide ancestry testing, such as Texasbased Dynamic DNA Laboratories, who that their testing allows you to "discover who your ancestors were" and "connect with relatives from around the world" (Dynamic DNA Laboratories). 
makes the sharing of this most intimate information seem more normal and harmless than it might be. It is also through this ethos and normalization of sharing that the highly individualistic and personal nature of the test results gains a dimension of collectivity and collaboration. ${ }^{17}$

Very broadly, Marina Levina argues that personal genomics takes "advantage" of social media technology and network subjectivity - the idea that individuals are always already part of and contributing to a network through the sharing of information to promote new, collaborative forms of doing research: Personal genomics, she argues, is discursively positioned as part of a "social-networking culture" in which a perpetual sharing of the self is standard, so much so that the generation of data and sharing of information is positioned as an act of (biological) citizenship $(1-2,5)$. Similarly, Delfanti argues that their social media websites and "discourses of participation and inclusion" are used by genetic testing companies like $23 a n d M e$ to encourage "active engagement with genetic research" (Biohackers 46-47). In 2009, for example, 23andMe proclaimed their so-called "Research Revolution" - "a Do-It-Yourself Revolution in Disease Research" - to "empower more people to jumpstart genetic research into the diseases that affect them and the people they love" (23andMe, "Introducing"). The aim were large-scale genomic studies for ten diseases in which people could participate through a special "23andMe Personal Genome Service Research Edition" that also gave them insights into their own genome, a cheaper version of the test at that time. This "research revolution" marked the first time that 23andMe tried to draw customers into genetic research. In 2018, 23andMe's information page on research claims that they give people the opportunity to "becom[e] part of something bigger" and "make a difference by participating in a new kind of research -online, from anywhere" (23andMe, Research; my italics). By answering online surveys and connecting them to genetic data, researchers can use customer contributions to "help drive scientific discoveries" (23andMe, "Research"). ${ }^{18}$ 23andMe, according to Levina, is one of the examples that introduces a form of "research 2.0," a model that creates and sells citizen bioscience: "a community building research initiative that promises to put individuals in control of their - in this case - genetic data and provide greater ability to affect scientific research" (3).

This type of citizen science promoted by $23 a n d M e$, of course, could be seen as empowering people to take part in research but it also is a form of appropriation of its ideals in which individuals relinquish their control over their data to the companies in question. According to Turrini and Prainsack, despite the company's "strong rhetoric around participation and democratization, the degree to which the company is transparent to its users is very different from the degree to which its users are transparent to the company" and control over how the company uses their data is limited (7). In fact, in this type of citizen bioscience, individuals are used as a source of "free labor"

17 The personal nature of the results, however, also has a collective dimension if we look closer at how those results are created: The Genome Wide Association Studies (GWAS) that form the basis for many of the correlations compare genetic variants and phenotypes between large cohorts of the population - they therefore always have an element of collectivity.

18 The patient-consumer is free to "opt-out" or "opt-in" to this type of data use at any time, claims 23andMe. 
(Levina 5) in the biomedical market, a free research commodity facilitated through a discursively created (moral) obligation to participate and share information (Delfanti, Biohackers 46-47). The access to their biology that people grant the companies as well as the labor they perform - pricking blood, collecting saliva or other biological specimen - become "biological resources" that produce "economic value" (Harris et al. 73). A noteworthy example of such value-production from genomic data is the cooperation between 23andMe and GlaxoSmythKline (started in 2018), in which they use genetic samples out of the former's large genetic data base and filled out questionnaires to find possible targets for new drugs - that could then be used for commercial development (23andMe, "Note Collaboration"). This is not just questionable from a data privacy standpoint but also ethically: The only people, who will not benefit from this arrangement financially are those who opted-in to sharing their data for research (cf. Ducharme).

A strong incentive to participate in this type of free labor, according to Turrini and Prainsack is the possibility to contribute to collective scientific knowledge. They claim that for many respondents in empirical studies, curiosity about new technologies and a keen "interest in participating in biomedical research" where motivational factors for their use of DTC testing (5). The discourse of participation and collective impact is also employed by 23andMe: "With the help of our 23andMe community we believe we can accelerate research and make an impact with our genetic data." (23andMe, Research, my italics). Accelerating research (possibly helping other people) and making an impact (leaving a mark) are used as incentives for customers to 'opt into' research. One factor in Turrini and Prainsack's discussion of the several layers of utility or value of DTC testing for the customer, thus, is a social one, both by creating new forms of identity articulation towards and in relation to others and by creating larger social value by contributing their information to genomic databases and research efforts (6-7). ${ }^{19}$ The largescale studies that $23 a n d M e$ is helping with, they claim, "are poised to transform medical research and empower more people to join more studies" (23andMe, "Research"). All this is buttressed by a "rhetoric of scientific democratization" (Turrini and Prainsack 6), in which customer-patients are active contributors to scientific advancements as well as proponents for a new, open form of doing science.

While it is far from open-source approaches, DTC (genetic) testing is here aligned with discourses of citizen science, empowerment, participation and democratization of research - making it accessible, allowing also 'lay' people to contribute what they want and can. Here, a commercialized form of DIY focused on individual choice and self-administration is discursively merged with amateur biological research. A somewhat related example is the Personal Genome Project: This non-commercial project strives to make whole genomes of participants accessible for researchers and the public alike.

19 Turrini and Prainsack, in their discussion of the utility of DTC genetics, argue that taking a DTC test is not the individualistic activity it is often seen as but done with or with reference to others: friends, family, significant others, society as a whole. "In this sense," they claim, "genomic information is personal and social at the same time: it is personal, but for more than one person" (5). The value of personal genomic information for them is thus "intrinsically social" (7). Moreover, the new forms of identity articulation have a parallel in the emergence of standardized clothing sizes: They prompted individuals to rethink the self and body in numbers - numbers that invited and were created through comparison with others. 
Participants must demonstrate that they do indeed understand the basics of genetics as well as the repercussions that the sharing of their genetic and trait data might have for them, because no anonymity is promised (Harvard Medical School/Wyss Institute). While this is not exactly a DIY technique, indeed rather a highly mediated one, it is nonetheless a perfect example for genetic citizen science based on open-source principles. It is the exact opposite of those closed off, inaccessible databases of the genetic testing companies, a sharing for the greater good instead of company profits.

Still, DTC testing has found its place among DIY biologists, in their practices and cultural representations. For example, journalist Marcus Wohlsen includes personalized medicine and DTC genomics in his discussion of Biopunk (2012). Wohlsen dedicates a chapter to the increasing ability to "read" DNA, amongst other things through DTC tests offered by companies like 23andMe. In this chapter, he includes the story of Raymond McCauley, a bioinformatics professional, who in his free time has turned to DIYbio and DIY solutions for his passion projects and potential innovations: To satisfy his curiosity as a "hacker," McCauley took a DTC genetic test to find out more about himself. Not only did his results have actual material consequences on his body - he lost seventy pounds to improve his odds for some of the more worrisome test results - but he did not stop there: McCauley connected with four other "gene hackers" to start a DIY clinical trial to find out if a mutation in a gene McCauley carries may impair his body's ability to use folic acid. Spread throughout the US, the four 'test subjects' went to an online doctor to get a prescription for a blood test which they had done in nearby clinics. After receiving their initial results, they started a period in which they "washed out" folic acid from their bodies before testing the effects of both a regular off-the-shelf product and a bettertailored vitamin, checking their folic acid levels regularly for comparison. The results showed that McCauley's body could process the tailored vitamin better. Apart from this personally useful piece of information, for McCauley the "most powerful achievement of the so-called citizen science effort was the fact that it could be done at all" (Wohlsen 13340). ${ }^{20}$ Without cheaper access to one's own genetic data through DTC tests, Wohlsen argues, this type of citizen science and biohacking effort would not be possible. He predicts that with the new tools at our disposal, amongst which he counts not only DTC options but also crowdsourced data bases and genetic interpretation programs, consumers might in the future become DIYers (121). For him, thus, DTC and DIY are not two distinct approaches; rather, they can influence and inform each other, be combined in DIY biology projects in a way that is meaningful and practical for the 'biohacker.'

20 DIYgenomics, a non-profit research organization that wants to realize personalized medicine through crowdsourced health research studies, is an online platform that uses approaches such as McCauley's on a broader scale. Since 2010, people can choose studies and participate in them, by trying a set series of interventions and measuring the results with blood tests, including for example studies on vitamin deficiency, aging and mental performance (DIYgenomics). After receiving more press coverage in 2011/2012, it has been quieter around DIYgenomics in the past years - their website and studies, however, are still active. 


\section{Case Study VI: Make it Personal - Do-lt-Yourself Testing}

„In a sense," Wohlsen writes, "we will all become DNA tinkerers, making decisions about our lives and lifestyles in an effort to tweak our futures based on what our genes appear to have in store for us" (125). "DNA tinkering," in this case study, is taken much more literally as we turn to Wohlsen's representation of a biohacker's efforts to DIY the genetic testing process. This account is dominated by considerations of access to knowledge, self-understanding and self-awareness, as well as, interestingly, bodily modifications and self-enhancement. In this case, the DIY testing process becomes truly personal: The test is conceptualized, implemented and analyzed by the individual affected and the results are poised to confirm or deny the existence of a genetic mutation in the tested and testing individual themselves, personal knowledge about personal risks. The goal is not a complete genomic picture but knowledge about a specific mutation. The narrative Wohlsen includes is that of Katherine "Kay" Aull, a professional bioinformatician with a background in synthetic biology, cancer research, and immunology, who in 2009 at 23 years of age set up a biology lab in her closet in order to test her own DNA. Aull's story, apart from providing insights into the DIY biology movement, has become a staple reference point for both journalistic and academic authors - and it is also this prominence that has led me to include her story as well. Let me first recount what happened, following Wohlsen's narrative.

In 2008 Aull had received her Bachelor of Science in Biological Engineering from the Massachusetts Institute of Technology (MIT) and afterwards started to work in a wetlab for a synthetic biology company in Cambridge, MA. It is here that her story unfolds, a small apartment in Cambridgeport that Aull shared with three roommates and her cat. ${ }^{21}$ Using rather "basic tools" (Keulartz and van den Belt 7) Aull set up a wet-lab for roughly $\$ 1000$ that would both fit into her closet and could be used to test her own DNA. Why did she go to that length? Aull wanted to find out if she carried a genetic mutation connected to hemochromatosis, a hereditary disease common in the US. In Kay Aull's family, the men tended to die young, narrates Wohlsen. When her father was sixty, his doctor noticed high counts of liver enzyme. When his numbers did not go down but increased despite alcohol abstinence, a blood test revealed something unusual: thick and viscous blood that caused the doctors to perform more tests. Connecting the thick blood and grating joint pain her father had experienced for years, he was finally diagnosed with an advanced case of hemochromatosis, a condition that causes the body to absorb and store too much iron, which builds up and leads to chronic damage. The treatment is simple: Since his diagnosis her father had to give blood every month to remove the excess iron (13). About ten years earlier, researchers had isolated the gene for iron absorption and its mutations causing hemochromatosis, making this hard to diagnose condition easier to identify without a doubt (13). This test is expensive and usually other possibilities need to be ruled out before an insurance company would pay for it (14). But "Aull wanted to know if she ran the risk of contracting hemochromatosis too," writes Wohlsen. So instead of waiting and monitoring her health or demanding an expensive genetic test, she decided to "swab her cheek with a Q-tip, mix it with her 
primer, and stick it in the thermal cycler in the closet" (Wohlsen 14-15). Using tools from her kitchen and second-hand gear from eBay, Aull built a gene test herself: In an effort of truly personal genotyping, she decided to extract her own DNA, amplify it, and using a genetic primer ordered online detect possible mutations in her DNA. ${ }^{22}$ She bought an "antique" thermal cycler on $e$ Bay, used a rice cooker and whiskey tumbler to make distilled water, and even tried to build her own electrophoresis box from a picture frame and a plastic box (Delgado 70; Keulartz and van den Belt 7). The test was successful, it worked. But it also showed that she indeed carried the mutation. According to Delgado, "Aull wants to have control over her future, and she wants to have it now. So a sense of personal history comes together with an aim at anticipating the future and taking action now" (70). Kay Aull did not want to wait but instead let herself be guided by her biological curiosity to create a test 'on the cheap' herself - a typical narrative frame for biohacker stories.

In this description, we find many elements of a DIY or hacker approach to biology. Aull had to think about what she needed, get or make the equipment, prepare and perform the test and interpret the results herself. Her aim was a cheaper but functional solution, an alternative to expensive tests prescribed by medical professionals under certain preconditions, to show that it is doable in a 'home lab' - and she also wanted to know something about herself. Wohlsen emphasizes the 'hacker approach' by including not just the tools and setting but also Aull's motivations and mind-set into his narrative, to which I will come later. As is common for DIY biology, the tools she used are simple solutions, either makeshift or second-hand: A rice cooker, a whiskey tumbler, a used thermal cycler, and other household items. She used what was readily available and profited from the availability of used gear after the last economic crisis: the new, as Morgan Meyer called it, "citizen biotech-economies" emerging through the redistribution and democratization of material resources ("Build" n.p.). ${ }^{23}$ The setting, as is typical for 'garage biology,' is domestic, a kitchen, an apartment in this case. Wohlsen highlights this domesticity when he describes her apartment in "a traditionally working-class neighborhood in Cambridge downslope from MIT along the Charles River," in which "[t]he lab shared space with her three roommates and her cat" (14), who works as "her chief safety officer ('If he can't play with it, I can't either')" as well (11). Close to but also distant from the elitist science at MIT, Aull's experiment does not take place in the confines of the 'ivory tower' but in a personal space, a shared space between different people and animals. This setting emits a certain casualness, a harmlessness of this type of DIY testing. Her cat as the "chief safety officer" also conforms to this image: The materials she uses, in general, are safe for living beings - a certain form of self-regulation often propagated by the DIY biology community. However, more than in DTC tests, in this case a kitchen, a home is turned into a laboratory, not just for the collection of a biological specimen but for the whole of the testing process. In this reframing of the home, such molecular tests also gain a spatial dimension: Close to home, they are

22 This primer is designed to bind to genes with the mutations for hemochromatosis, resulting in longer strands of DNA that can be made visible using a process called gel electrophoresis in which DNA strands are sorted according to length. 
a symbol of convenience, access, empowerment, but they might also get too close for comfort. Not just that the public once more impinges on the private (or that through the obligation to share the private is made public), but also the results might be too close to home, the own body, for comfort: they might reveal uncomfortable truths. This is true for DTC testing as much as for Aull's DIY solution, its experience - the vulnerabilities, obligations, uncertainties and subjectifications - as much as its public evaluation. ${ }^{24}$

Kay Aull is by far the only DIY biologists who has performed genetic experiments on herself. In fact, for DIY bio community labs the extraction of workshop participant's and DIY beginner's DNA is a common experiment - Delfanti writes that in New York DIY biologists were "extracting and genotyping people's DNA at public events" (Biohackers 116) - and other experienced bio-hackers claim to have performed genetic therapies on themselves (cf. Quartz). But Kay Aull has become a staple reference point for journalists like Wohlsen and academics interested in DIY bio. Most academics are aware of her fame: Delgado, for example, acknowledges that Aull has gained a lot of media attention for developing her "homemade genetic test" $(70) .{ }^{25}$ One possible explanation for this fame is that her experiment was performed quite early in the DIY bio movement's trajectory: In a phase during which the movement was still finding its place and garnering support, a noteworthy experiment like hers was bound to draw some attention, also among the hackers themselves. Moreover, Aull is also emblematic of the whole movement: Like many of the early (and current) DIY biologists, she has a professional background in biology and the respective insights into how to plan and perform such an experiment. For her, it was more of a question of 'downsizing' the needed equipment than of gaining access and scientific literacy to do it herself. Aull is not a "novice" as Delgado calls it (70). Her MIT degree is also often included in references to her 'hack,' so one could also argue that this institutional authorization is appealing for journalists and the media: She knows what she does and has the credentials to prove it.

However, apart from her scientific background, Aull's story is also a deeply personal one, full of genetic responsibility and biosocial connections. Visible in Wohlsen's account, her 'self-experimentation' combines a DIY ethos with a personal 'tragedy.' In his narrative, he seems to endow Aull with four different identities: an "evil genius," a professional biologist, a hacker/tinkerer, and a personal victim. Wohlsen starts his narrative as follows: "For an evil genius, Kay Aull comes off as very self-effacing. In fact, the twenty-three-year-old MIT grad has no malicious intentions, unless you consider her desire to grow a tail a crime against nature." (9) This positioning as an "evil genius," a nice and modest one at that, seems to combine her identities as a hacker/tinkerer and professional scientist: "She also speaks with the crisp confidence of someone accustomed to knowing more about her area of expertise than anyone else in the room - and she probably does." (9) Wohlsen acknowledges her expertise that does not just come from being the one to do this test but also from her educational background.

Wohlsen also thematizes the contradiction between the home and lab as spaces where biology is practiced, drawing on the common evaluation that the home is not a safe space for molecular agents (18). For more on Wohlsen's different valuations of the spaces of biology cf. Case Study IX. cf. also Wolinsky, Keulartz and van den Belt, Meyer ("Hacking"). 
However, it is the focus on her family history and the personal nature of her DIY endeavor that makes her most relatable for the reader. We find glimpses into how the rest of her family coped with the diagnosis: from her parents' search for information online to Aull testing herself. The medical background of the story and her personal stakes in it, allow Aull to also be framed as a "patient advocate" (Jen 134). Her experiment, thus, combines a new trend with an old narrative frame, one that is inherently human: the repercussions of a new medical diagnosis.

In Wohlsen's narrative the result of the test seems to turn Aull into a somewhat tragic figure, torn between the implications of what she now knows and the scientific spirit that led her to the discovery, her emotional reaction and her rational approach as a scientist:

Aull said the mutation she carries still means that there is a less than 50 percent chance that she will contract the disease. Yet I could see her enthusiasm for the test was blunted as she talked about the results. As with genetic tests for Huntington's disease or Parkinson's, Aull could do little about what she found out other than watch and wait. She is the same twenty-three-year-old she was before the test, but now genetically haunted by a possible future over which she has little control, except for knowing to tell a doctor about her genetic pre-disposition if suspicious symptoms appear. 'Everyone has these deep dark genetic secrets. That's just how it is,' Aull said. 'Knowledge is complicated, but ignorance is not better.' (Wohlsen 15 , my italics)

The reality of the test, it seems, had "blunted" her enthusiasm. In this quote, Wohlsen's impressions seem to dominate the account and he seems to make use of a common narrative of genetic testing: the patient-in-waiting, the uncertainty that arises out of genetic knowledge, especially with tests for diseases that lack a cure. In Wohlsen's account, the test that Aull herself sees as a form of empowerment becomes a genetic "ghost" that will haunt this young girl for the rest of her life. An act meant to take control makes her lose control. The value of such a test suddenly seems to be ambivalent. Likewise, for Delgado the result of the test means that "her present seems to be trapped in between her genetic past and her risky future" (70). Both journalist and academic seem to draw on the same basic narrative of genetic risk. But for Aull herself this narrative seems to be beside the point: For her, satisfying her curiosity seemed to come with an inherent warning that she has to accept, even embrace, as a scientist: Knowledge, no matter how complicated, is always better; what counts is the experiment, the solution, the hack. Aull's own voice seems to be drowned out by culturally sanctioned narratives and expected reactions that stand in contrast to - or cannot yet integrate her overarching goal and ideology. ${ }^{26}$

However, Wohlsen nonetheless seems to endorse personalized medicine and genetic testing, also self-made testing, as means to gain in self-awareness:

Aull sought to learn something profound about herself. Through sheer inventiveness, she tinkered her way to that knowledge. She hacked her genes, and she gained in self-aware-

26 We could, of course, also do a feminist reading of this passage in which the male narrator silences the female voice and subordinates her reading of a situation to his own. 
ness. Perhaps do-it-yourself biology will someday mean a new kind of introspection: the ability to self-examine with more depth and precision than Socrates could have dreamed. (Wohlsen 16, my italics)

From tinkering and hacking, from material practices with rough edges and workarounds, he seamlessly moves to broader issues of self-awareness and self-understanding. DIY tests, he speculates, could become avenues for profound self-examination reminiscent of previous forms of introspection. The material equivalent of Socrates' 'know thyself. ${ }^{27}$ By including this reference to internal forms of self-examination conscious reflections on one's sense of self and mind - Wohlsen also seems to endorse some form of genetic essentialism: The results of a gene test can reveal to you your inner essence, your inner truth more precisely than ever before. Also in a cultural representation like Wohlsen's account of Biopunk, self-knowledge and self-reflection seem to be an intrinsic part of the testing process: This, of course, repeats the discourses of self-knowledge so present also in DTC testing, truth about the self wrought from the material body.

Wohlsen, however, does not stop there. Instead, he chooses to end his chapter on a notion that he implies at the beginning: Self-modification, self-enhancement. There is nothing in her experiment that suggests any form of enhancement as the end goal, but still he arrives at that conclusion. A somewhat lengthy quote shows how he connects DIY biology with the ability to "write" genes, thus making biopunk phantasies of enhanced or altered bodies seem in reach:

Perhaps DIY biotech also means a new kind of freedom, where hacking your way to greater understanding of yourself is just the first step. Synthetic biology promises the ability not just to read genes but to write them, like printing out letters on a page in a pattern that creates a picture no one has ever seen before. In biophrophet's wildest imaginings, hacking human genes could mean making yourself more than human. Then again, inventing ourselves anew is the essence of individualism. Maybe giving ourselves tails or wings or chlorophyll-covered skin is just being human, fully realized, free to make ourselves into whomever or whatever we want to be. Maybe that freedom means we will not have to wait for nature anymore. This may or may not be desirable, but these are the dreams that stoke the biopunk imagination, fueled by Blade Runner, radical libertarianism, Newton, Darwin, and a fierce will to power and transcendence. (Wohlsen 16-17, my italics)

In Wohlsen's account it seems like self-enhancement, "making yourself more than human," flows naturally from self-knowledge. In fact, he argues that "inventing ourselves anew" is deeply imbricated in our notions of individualism: The freedom to choose whoever we want to be. Biotechnology, or DIY biology as its most individualistic and open form, could give us the freedom to do so by also altering the material basis of our

27 "Know thyself!" - this maxim of self-knowledge, of course, has a long cultural history: from the

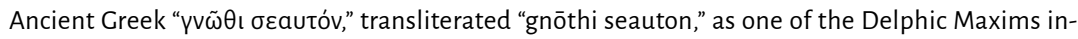
scribed in the forecourt of the Temple of Apollon at Delphi to the Latin the phrase "nosce te ipsum" and their uses by Franklin, Rousseau or Emerson, to name a few, it has been part of a long legacy of philosophical debate and thinking. 
bodies. He does, however, hint at that conclusion, thus preparing the reader, already in the opening lines of the chapter when he references Aull's desire to "grow a tail." This paragraph also shows the close connection between DIY biology and transhumanism, also in the cultural imaginary.

What is even more telling is that this chapter is not the only one that ends with the transhumanist-biohacker's 'dream' of modifying and enhancing the human species. It seems, that what Wohlsen in part wants to convey is that self-modification is the ultimate goal behind biohacking and DIY biology but also behind society more generally. His reference to the cultural ideal of individualism tells us that he does indeed see this fantasy as a result not just of technological innovation but also of the direction American culture is headed more generally. What is more, Wohlsen thus provides us with a perfect example of how DIY biology, medicine or biotechnology is intrinsically connected to ideas of self-enhancement in its practitioners, its representations and cultural discourse more generally: The logical conclusion to all kinds of new knowledge or new technologies seems to be a way to use them to serve our human inclination to make ourselves better than we currently are. DIY biology and medicine, Wohlsen implies, are no exception here.

While this DIY approach and the DTC examples above are two sides of the same coin, a DIY test nonetheless represents a new epistemological approach to how science is conducted, a practice-based, experimental one. To set the test up, according to Wohlsen is also an intellectual and creative challenge. Wohlsen writes, "[Aull] clearly relished the challenge of doing the work at home - for cheap, relying only on her wits and creativity." (11) Aull "challenged herself" to build that test only with the makeshift tools she had (14). Her mind-set is that of a maker and hacker: affordability, ingenuity, creativity, fun. This approach is described by Wohlsen as a different form of practicing science: "a more pure kind of science, a curious mind engaged passionately with nature, free of any of the most common ulterior motives" of career, profit or prestige (11). It is an idealist, utopian version of science in contrast to that represented by the looming shadow of MIT, her alma mater down the Charles River. This type of science is portrayed as a return to her origins, the amateur beginnings of all future scientists: "By resurrecting her inner nine-year-old, the girl who cross-bred houseplants while her peers played Nintendo, Aull appeared to be purposely stoking her primal scientific impulse, the driving energy of discovery." (Wohlsen 11) Wohlsen describes Aull as a scientist by nature not just by training. In pursuing her own research, she is returning to her roots, the impulses of curiosity that once drove her to 'discover' science as a hobby and profession. For her, Wohlsen cites, this process is inherently connected to a 'do-er' ethos: it is not just about understanding but about doing it, "poking it with a stick" (11). As a 'purer,' more practical approach, such a DIY set-up is also poised to provide new knowledge, not just about the self but also about how science can be approached as an everyday occurrence.

For Aull her DIY test is a form of demystification: "I think the most important thing about DIYbio is it's something you can do too. It's not magic. It's chemistry,' Aull said. 'Doing it in the sink demystifies the process."' (Wohlsen 14-15, my italics) Here Aull does not just perpetuate a mainstay of the DIY movement - that you can do it yourself - but also speaks to the broader social relevance of DIY biology as a form of science com- 
munication: Turning it from opaque "magic" into a daily occurrence. This process of demystification also shines a light on another distinguishing factor of a DIY set-up that connects back to the question of literacy. When the testing process is controlled and carried out by the tested person themselves the individual has to reach a higher level of literacy where they do not only understand the biological basis but can actually do biology. If you manage to genotype yourself chances are high that on the way to get there you picked up enough knowledge to properly contextualize the results. A DIY testing process thus might obliterate the concern that people cannot properly understand the results they get from DTC tests.

What it comes down to, therefore, is scientific literacy, the ability to not just understand but perform biological experiments. This type of literacy or engagement with medicine/biology starts with the collection of biological knowledge from online sources like Google - a means to educate oneself that Aull's father pursued - and reaches into the actual performance of genetic tests. Aull, Wohlsen claims, thinks people are responsible for knowing, for taking advantage of the decades of research and information that are out there for everyone to find (cf. Wohlsen 14). DIY experimentation's transformative potential could thus also mean a broader public understanding of science. While the hacks he discusses are practical examples of learning-by-doing, in fact Wohlsen's book can be read as his own attempt at science communication and translation, at increasing the scientific literacy of his reader: He is eager to give explanations or translations of what Aull did, to make the process of testing and its theoretical premises understandable also for a lay audience. To do so, he does not shy away from a scientific explanation of the history, theory and practice of gene splicing, though in a rather unscientific language (apart from specialized key terms). Here, he resorts back to the old and trusted language metaphor of DNA as a 'genetic alphabet' and simplifications in the name of comprehension.

However, we also need to acknowledge the potential for stratification inherent in a 'start-to-finish' DIY test. In theory, DTC tests are an easy - and today increasingly affordable - version of genetic or molecular 'experimentation' that many people can carry out without specialized knowledge. DIY on the other hand, as of now is rather elitist: DIY's goal of making it accessible for everyone is not yet the case. Some crowd-sourced endeavors and start-ups, however, strive to change that. One example is Promethease, a literature retrieval system that uses "SNPedia" - an open-source wiki for human genet$\mathrm{ics}^{28}$ - as the foundation for personal DNA reports. If you have your raw genetic data, for example from a DTC testing company, you can use Promethease to learn more about your DNA, independent of the company and their interpretation. For $\$ 10$ you can get what I would call a 'second opinion' on your genetic data in the form of your own, independent DNA report. Both of these tools, in fact, were good DIY alternatives during the time in which 23andMe was prohibited by the FDA to reveal genetic health information: everyone who has a scientific reference for the information can put in new SNPs. Dolgin claims that SNPedia can help people to get more information from their genetic data than the interpretations by the companies (955). 
You could just upload your raw data, which of course included that information, and run it through Promethease or look up variants on SNPedia - if you did not mind the extra work you had to put in yourself. These types of services can provide users of DTC tests with more knowledge on the cheap, thus constituting a middle road between DTC and DIY testing.

\section{To Summarize}

As a practice of DIY biology and medicine, testing the molecular make-up of the body is portrayed in cultural representations as a path to new insights into the self and potentially new means to optimize and improve one's health, wellness and life, and this is true for commercial and maker solutions. These tests, their process and insights - the new 'molecularized self' they promise to reveal - become starting points for personal storytelling. In their study of DTC testing, Harris et al. look at YouTube videos shot and posted by individuals who did DTC genetic testing for disease: They performed narrative analysis of these videos and found a new form of storytelling they refer to as "autobiology," "the study of, and story about, one's own organism" told on a molecular level - selfmaking and sensemaking through biological practices. Autobiologies, they argue, are characterized by their playfulness and casual engagement with the technology but also bound up with consumer culture (61-62, 73). The settings of these videos, interestingly, for them become "at-home biological 'laboratories"' - referencing the domestication of biological labor and research so prominent in DIY biology and medicine. However, this playful and fun experience with biology can also have negative sides. Molecular testing, also in the rhetoric used by the companies, participates in and perpetuates discourses of self-knowledge, personal responsibility, and prevention. Gaining molecular knowledge of the body, through testing, is coupled with an obligation to react to the results, to change one's body, one's lifestyle to better alleviate risks and promote health. This responsibilization runs the risk of perpetuating an ableist culture, with a hyper-focus on able bodies, stratified between those who can afford the testing and those who cannot.

Still, the case studies in this chapter have demonstrated that commercialized interventions and hacked solutions are not two opposing ends of a pole. Rather, they can be fruitfully integrated into each other, as the cultural artefacts under analysis have shown: Direct-to-consumer testing is rhetorically and ideologically positioned as a possibility for participation and citizen science, while Wohlsen's account connects a DIY test back to values of individualism and enhancement so prevalent in representations of DTC testing. In the cultural imaginary, such practices are enmeshed, work with and alongside each other. They are different routes to a similar result. Where they stand apart are the levels of biological literacy needed to succeed (with the concomitant possibilities for stratification): Are people in DTC testing settings presented with enough information to set their results into context? Do they have enough (genetic) literacy to do so? When people opt for commercial tests, it is not necessarily a given that they can, for example, interpret the results of a gene test correctly, set the risk ratio they are given 
into context. ${ }^{29}$ Kay Aull's example shows that a DIY test needs a certain degree of scientific literacy or a setting in which a lay individual is provided with both. Community laboratories could provide such spaces.

While a DIY test like Aull's is currently probably not on the road to mainstream, it nonetheless has symbolic potential. Keulartz and van den Belt argue that the test, of course, is not a scientific break-through but that its main premises - reduction of costs for biotech equipment - can have a "transformative impact" on how society deals with science (7). Wohlsen narrative includes a similar claim: The drastic reduction in costs reflects a "change in sensibility." Aull's test is not a break-through or drastic innovation but "a new way of doing science:" "A practical piece of biotechnology" that though using some of the most sophisticated science of its time was built at home, in a closet, with cheap tools (Wohlsen 15). It is, to relate it to the overall DIY movement, not new but experienced as new, gains a new quality. Wohlsen connects this development to a wellknown example all of us have experienced ourselves: Mobile phones. Once they became cheap, they became ubiquitous and changed the expectations of communication and reachability in US culture and world-wide (16). Digital technology transformed culture "not only by what it could do but by how cheaply it could do it" (16). By choosing this example Wohlsen not only makes the processes easier to relate to for the reader, but also acknowledges the cultural force of biotech, especially its democratizing potential. "The power of Aull's project," Wohlsen writes, "lies not so much in what it can do but in how little it cost" (16). In Wohlsen's narrative the DIY approach becomes a symbol, whose potential, similar to the DIY EpiPencil, lies not so much in the project itself but in what it signifies. 



\section{Homo Technologicus? - The Technological Self, Inside Out}

Self-tracking technology has taken the consumer market by storm during the past few years. Used to 'track' or 'monitor' the self, these tools promise unprecedented access to the individual body, possibilities for self-improvement and new forms of bio-sociality. The wearable tools of the quantified self are emblematic of how humans interact and connect with technology. They are increasingly integrated into one's sense of self and the surface of the body. In the visions of an enthusiastic community of body modifiers as well as global tech corporations, similar technologies will soon be incorporated into the body. ${ }^{1}$ At the moment these practices and visions of technological immersion human-machines, turning Homo Sapiens into 'Homo Technologicus,' where self and body are thought of in technological terms - are still primarily practices of DIY.

Such visions, however, are not particularly new. The impulse to track, to modify and merge with technology has preoccupied human imagination for centuries. Observing ourselves has always been a concern for humans, self-reflection and introspection have been practiced from the old Greeks to the Puritan settlers of America. As many scholars of the "Quantified Self" have argued, today's forms of tracking the self go back to older forms of keeping track of parts of our lives: diaries, body measurements, household books, personal budgets have all been used to record, plan and analyze everyday life (Lupton, Quantified Self 4; Abend and Fuchs 6). These analogue, language-based forms of "keeping track" were soon supplemented with measurements and numbers. In this sense, we have always been quantified to a degree, the technologies, tools and possibilities however have changed. The rise of computing and digitization have made possible new forms of tracking that allow for a detailed, minute and automated monitoring of 
the body and life (Lupton, Quantified Self 4). In this process, introspection was replaced by technologies that penetrate even deeper, not only into the mind but the body: trackers, sensors, blood tests, that can give - even daily - insights into its status quo; external monitoring of quantifiable data. ${ }^{2}$ But also visions of cyborgs and man-machine hybrids have been part of the cultural imaginary since at least the $19^{\text {th }}$ century - for example in Edgar Allen Poe's “The Man That was Used Up" (1843) - and automata, complex, sometimes humanoid machines, have sparked people's imagination and inventor spirit since Greek mythology. While tracking the self has been part of everyday practices, cyborgs, hybrids, androids, on the other hand, have for a long time primarily been the subject of (science) fiction.

In its most common understanding, self-tracking refers to practices in which people use apps and gadgets to create (self-)awareness of their lifestyle and improve it in almost all areas that influence life quality and health. The most common technology used to do so are wearables such as activity trackers and smartwatches that are based on (nowadays cheap) acceleration measurement and optical sensors (Schumacher 4042). These tools range from simple trackers that count steps or quantify a day's physical activity to those for health issues such as glucose levels, blood pressure or heart rate; for meals and calories; more specialized applications such as tracking of ovulation and menstrual cycles, quantification of sexuality and pregnancy apps; the tracking of environmental factors, geolocations, personal encounters and work productivity; to the recording of emotions, moods and affective states. Some of these tools are worn on the body, while others are digital tools and apps that require manual input or track automatically. The plethora of tracking technologies - together with the rise of the "internet of things" - shows that today we are embedded in an "ecosystem of smart devices" (Bietz and Hayes 84) that could potentially "monitor" all aspects of our daily lives: what we do, where we go, what we eat, whom we talk to, how we feel, and what our bodies do at any given moment. As these trackers become even more pervasive and embedded in people's daily lives, chances are high that their acceptance will rise even more and tracking the self becomes a normalized, even routine and required part of daily life. 'Monitors' could become our daily companions, outside and inside the body.

According to Lupton, mobile digital tracking technologies produce a "spectacular body," make internal workings visible. They attempt to penetrate the "dark interior of the body" in the name of security and stability: by making it visible - as graphs, lines, and data points - they attempt to make it knowable and manageable ("Self-Tracking Cultures" 5-6; Quantified Self 54). In so doing, tracking also promises control of the 'untamable, the unruly biological nature of humans through its conversion into objective, neutral, manageable data. Abend and Fuchs argue that the data generated by self-tracking technologies are "framed as direct access to a truth about the self," the promise that

2 Many scholars of quantification point out that data are perceived as objective and (value-)neutral: a higher authority (Lupton, Quantified Self 56), unaffected by human failings (Bietz and Hayes 82), more reliable than instinct and physical sensation (Lupton, "Self-Tracking Cultures" 5-6). Some of this perception comes from their foundation in 'objective' systems of knowledge production. In science data are seen as 'hard' facts, the truth, devoid of the researcher's interpretation or cultural influences. 
collecting, interpreting and comparing data enables us to access our true self (12). Our material body, our biology, here becomes a source, an access point to a deeper understanding of the self. Numbers and data are seen as providing a "route to personal insights and self-knowledge" (Bietz and Hayes 82). Chrysanthou argues that such techniques alleviate uncertainty by moving from risks and probabilities derived from the general population to evidence from one's own biology (474).

As such, data can, according to Lupton, be seen as productive, they generate new knowledge and new means of managing populations through individual bodies (Quantified Self 41-42). The tracking technologies used to gather these data can be considered as "disruptive technologies" (Coupette) - technologies that reach a high prevalence in society in a short period of time. Those carry with them the potential to challenge institutionalized rules of society and destabilize the "cultural matrix" by, for example, creating new social selection criteria and new principles of valuation and organization (Selke 54). Technologies, thus, can also have social and cultural effects. And in deed, the technologies, the devices used to track and monitor, are a crucial part of this contemporary amalgamation of bodies and data. Self-tracking is one of the easiest, pain-free, commercially widely available, and socially sanctioned forms of integrating technology into everyday life and the human body (cf. Lupton, "Self-Tracking Cultures").

This chapter looks at quantification technologies as part of the DIY biology and medicine movement. Not only does self-quantification offer new possibilities for the DIY patient, it is also part of citizen science endeavors and techniques of self-care. At the heart of self-tracking, Selke argues, is the belief that data are the basis for informed decisions: only with sufficient information - meaning data - people can actively shape their own future (56). In the logics of the quantified self, data lead to pattern recognition that leads to changes in behavior and lifestyle - an automated process of collection, recognition, change that constantly repeats itself. Self-trackers "attach" hard data to many aspects of their personal life; at the same time, self-tracking puts the power over them in the hands of the individual so that the self becomes a "manager" of those data (cf. Moore 402). A prerequisite for this trend is the idea that individuals can and should manage and enhance the self, their performance and productivity. It is also in this sense that self-tracking can be considered a commercialized, mainstream DIY activity, much like the DTC tests described above: In some cases, tracking becomes a DIY solution to fix a perceived gap in health care provision, inaccessible doctors, or lack of technology; in others, tracking is a means of gaining self-awareness to improve the self; in others yet tracking becomes a tool for self-study and DIY citizen science experimentation (cf. also Sharon 103). DIY experimentation, moreover, is also the primary concern of those body modifiers that seek to integrate technology into the body: They experiment on and with their own bodies and/or self-made technologies.

The case studies chosen for this discussion each look at how we as humans integrate technology into our lives and bodies, not by force or coercion but by choice. Case study VII focusses on the quantified self, commercial forms of tracking the self and the body with technology. My sources here include media representations such as a feature on Chris Dancy, "the most connected man," or personal descriptions by self-tracking journalists, but also commercial websites for tracking gadgets. These sources combine perceived neutrality with affective appeals. Case Study VIII, on the other hand, looks 
at hacked and tinkered approaches, many of which embed technology not on the body but into the body: 'Grinders' use technology to upgrade their bodies, using hacked solutions to also track the body from the inside. Sources for this case study are common and more extravagant 'hacks,' the self-descriptions of some key players in the scene as well as their representation in online media reports. What is interesting is that in cultural discourse these two types of using technology to track and upgrade the body are often thought together. For example, an episode of the TV show Dark Net, that looks at the dark web, technology and internet culture (Showtime, Jan 2016-May 2017), includes tracking with wearables and sensors as well as grinding into its consideration of how people "Upgrade" their bodies in the digital age (Jordan). While the former is a commercial, external form of interaction with technology, the latter is niche and invasive.

\section{Case Study VII: Quantifiably Me - Hacking the Body with Numbers and Data}

Tracking the self - for data, for knowledge, for change - has become a contemporary lifestyle. This lifestyle change, it seems, shares some of its most fundamental premises with the maker movement or DIY trend more generally: they rely on smaller, more advanced, portable technology and computing; network and sharing culture; active participation and access to tools and knowledge; a playful engagement with materiality; and a keen interest in the improvement and optimization of one's life, body and wellbeing. In this case study, I want to show that self-tracking is part of 'DIY biology' efforts, potentially even its most mainstream and widespread version. A variety of academic scholarship has already been written about self-quantification and its practitioners, amongst others as a practice of self-hood, biopolitical strategy and contemporary form of surveillance, or hopeful vision of future medicine. ${ }^{3}$ Dealing with it on a few pages will therefore necessarily seem incomplete. That is why I specifically aim to illustrate the connections between the quantified self and DIY biology. ${ }^{4}$

What interests me are not so much the users or devices, but how they are represented. Advertisements and popular accounts shape how those tools and techniques are used and perceived. For example, Dave Eggers' The Circle is one of the most prominent examples of the last years, book and movie, in which not just the integration of technology and sharing into contemporary life are taken to an extreme, but also tracking

Some sources include: Lupton (Quantified Self); Lupton ("Self-Tracking Cultures"); Abend et al.; Majmudar et al.; Schüll; Schumacher; Sharon.

4 I am here dismissing the distinction between the "Quantified Self" as organization and self-tracking more generally because they rely on the same principles of quantification, self-knowledge, improvement and sharing. The 'original' Quantified Self community and organization was set up in 2007 by Gary Wolf and Kevin Kelly in the San Francisco Bay Area, after encountering more and more people who engaged in some form of self-measurement, as they claim (Quantified Self Labs). The Bay Area provided fertile ground for the early forms of self-tracking because due the start-up culture and Silicon Valley it was populated with enough hip technophiles exhibiting the right culture of performance and play. Now there are chapters of the QS community in many parts of the world, QS meet-ups organized by different people in a variety of cities. The Quantified Self organization can serve as an example of the broader social movement, which is why I also sometimes use quantified self as a description of the whole phenomenon. 
becomes an important part the Circle's corporate culture: Employees all wear wrist monitors and as part of their corporate health plan swallow a sensor that tracks their health from inside their bodies. The dystopian outlook in this book seems to criticize contemporary fascination with quantification. In other popular accounts and advertisements, on the other hand, Tamar Sharon observes that self-tracking is positioned as leading to "unique insights about oneself" and by doing so promises to alter the relationship individuals have with their own health, bodies and selves (103). Also Abend and Fuchs argue that especially advertisements for wearables promise knowledge of the self as much as a route to its improvement (11). Some of this promissory discourse has been reflected in media representations as well, while in other cases coverage has been more cautious and critical. This critical media coverage, however, does not seem to undermine the use of and enthusiasm about self-tracking technologies. In any case, representations can give us insights into some of the more controversial issues of the self-tracking phenomenon while also demonstrating its inherent connection to other practices of DIY biology and medicine: Self-knowledge and self-enhancement, access and democratization, literacy and knowledge production, and individualism versus collectivity are some of the concerns that demonstrate the similarities between the practices - and I will discuss each of them in turn.

As many popular accounts demonstrate, the quantified self is positioned as an old practice in a new guise. "Extreme navel-gazing is probably as old as staring at the stars," notes Kashmir Hill in Forbes. "If this sounds weirdly narcissistic," she writes, we should keep in mind that the precursors of this movement - essentially old linguistic means of knowing and recording the self - have "a forebear in a quintessential American: Benjamin Franklin, the founding father of self-tracking" (n.p.). ${ }^{5}$ Self-tracking as a form of self-knowledge, thus, does not only have a long history of use and acceptance but also goes back to some of the core American ideals and values I have discussed in Chapter 4. Quantified Self founder Gary Wolf writes in the New York Times: "From the languor of the analyst's couch to the chatty inquisitiveness of a self-help questionnaire, the dominant forms of self-exploration assume that the road to knowledge lies through words. Trackers are exploring an alternate route. Instead of interrogating their inner worlds through talking and writing, they are using numbers" ("Data-Driven," n.p.). Both authors position self-tracking as part of a long line of techniques used to interrogate, express and 'know' the self: The difference, according to them, is the medium - from language, inherently subjective and affective, to numbers, objective and neutral.

This neutrality seems to endow data with a new authority: self-knowledge is 'scientified. "Self-knowledge through numbers," used by Wolf and Kelly as the motto of the QS community, became the 'mantra' for self-trackers around the world (Quantified Self Labs). Data are often positioned in a positive light, as a force to be reckoned with. For example, in an interview feature with Chris Dancy - who has become famous as "the most connected man" because he wears or uses between 300 and 700 tracking and lifelogging system at all times - Samantha Murphy asks herself whether she might "miss 
out" on "unleashing [her] own vault of data" by not using as much tracking technology (n.p.). "Unleashing" tracked data, it is implied, allows them to productively work for you. Lupton uses the concept of "lively data" to "denote the manifold ways in which personal digital data...are vital." This vitality, productivity, for her takes two forms: For one, they are vital in themselves by influencing people's behavior, sense of self, social relationships, life chances and opportunities. Moreover, being fundamentally about the lives of people, their bodily functions, behaviors, moods, emotions, relationships, "[d]igital data generate new forms of knowledge and new insights into people's bodies and selves" (Lupton, Quantified Self 5). By tracking the self, people become scientists and experimenters on their own bodies, their own "lab rats" (Lupton, Quantified Self 69). They plan, conduct and evaluate their own experiments, a DIY approach to knowledge production. Instead of solely being consumers or recipients of (scientific, biological) knowledge, self-trackers are actively engaged in the production of knowledge (Sharon 100), about the self and human physiology more generally.

Part of this quest for self-knowledge is also a prevalent discourse of awareness and mindfulness of one's body and mind (cf. Nafus and Sherman 1789). Self-knowledge is coupled to self-care. In the self-tracker's claims of "personal evolution, self-improvement and self-awareness," the quantified self seems to be a modern way of fulfilling the "Greek tenets of 'knowing yourself' (gnotthi seautón) and 'taking care of yourself' (epimelēsthai sautou)" (Abend and Fuchs 9). This is visible already in the title of an article written by Gary Wolf for Wired in 2009: Self-tracking also for him becomes an answer to the old paradigm of "Know Thyself." Tracking is framed as a valuable tool for understanding and managing the self. As such, self-tracking can be argued to go back to other forms of taking care of the self, communally and individually. A means of "performing, presenting and improving the self" (Lupton, Quantified Self 140), self-tracking can be understood as a Foucauldian "technology of the self:" They can be used to take care of, work on or play with the self, depending on the context and attitudes of their users (Abend and Fuchs 9). ${ }^{6}$ Like other techniques of DIY biology and medicine that I have discussed in previous case studies, self-tracking is a way of gaining awareness and knowledge of the self, of taking care of yourself in a more immediate and intimate way than previously possible. The body, this biological organism, is perceived as intrinsically connected to the sense of self, a form of biological determinism brought about by the penetrating glance of technology.

A crucial part of this, of course, is access to technology, data, the body. In this sense, self-tracking, like genetic and molecular testing, can be considered as a new way to peer inside the body, to make visible what previously was invisible or simply to arduous to

6 In fact, according to Lupton self-tracking is in agreement with the four types of technologies Foucault identified: technologies of production - people generating data on themselves - technologies of sign systems - the manipulation and communication of "symbols, images, discourses and ideas related to their own data" and the devices - technologies of power - voluntary participation in strategies of power designed to impose certain forms of conduct - and technologies of self "performing, presenting and improving the self" through self-tracking practices (Lupton, Quantified Self 142). 
track and monitor. This new access to tools translates into access to more specific information about one's body. Instead of being granted access by medical professionals, through self-tracking individuals gain authority over their data and, maybe crucially, their interpretation (Lupton, Quantified Self 94-95). Self-tracking, thus, can allow people more authority over their own bodies: In the interview with S. Murphy Chris Dancy refers to the ways in which all of our data are already tracked today, such as credit cards, smartphones, GPS systems, as "Big Brother." In contrast, "[w] hen you take control of it yourself, that's Big Mother, and that relationship is nurturing, kind and not controlling" (n.p.). Instead of being "surveyed" by corporations and government institutions, self-tracking allows you to use tools of data gathering for your own individual purposes and grants you control over your own data - data used to "take care" of yourself. Similarly, Gary Wolf emphasizes that self-tracking allows you to reclaim some of the power over your data ("Data-Driven"). ${ }^{7}$ It is no wonder then that empowerment and "taking control" are some of the main imperatives in tracking, also perpetuated in advertisements and on websites (Sharon 97). Similar to other DIY techniques, thus, self-tracking is concerned with control, access and authority. The self is at the center of the decisionmaking processes around their body, health and lifestyle, or so it seems. But also the opposition to elite and closed-off systems, that is discernable in Dancy's idea of "Big Mother," relates self-tracking to the hacker ethos of DIY biology.

In contrast to this appeal of control and authority, Hill describes her own inability to translate or use her collected data productively: "When I attend my first Quantified Self Meetup, I discover that other members share my excitement and perplexity: They don't know what to do with their data" (n.p.). The masses of collected data need be made sense of, need to be transformed into something that we can easily understand, that provides meaning for us, that we can use to act on the body. What is lacking also in parts of the quantified self movement, it seems, is data literacy, the knowledge of how to evaluate and use (their) data. Representing the tracked data, simplifying them, putting them into a visual, easily understandable form is necessary to make those data truly accessible also for lay users. As Wolf writes, "messy" data need to be translated into meaningful information through mathematics and computers ("Data-Driven" n.p.). Florian Schumacher argues that only with reappraisal and interpretation the measurements that form the basis of all data applications "make sense:" "through abstraction, interpretation, and representation data are turned into information, which can be developed into knowledge" (43, my translation). Only after this process of 'translation,' individuals can truly use this knowledge about the body. That, however, means that there always has to be an interpreting "authority," be it a human or machine (Schumacher 44).

Self-tracker Wolf acknowledges that in order to collect and use "neutral" data about the body we have to rely on machines: "We lack both the physical and the mental apmany arguments about data privacy and corporate control over collected data. Even though many companies emphasize their focus on data privacy and individual control, the tracked data are also embedded in a larger network of value production, sometimes even without the knowledge of the consumer. Data collection becomes a form of digital, free labor - for more on this see e.g., Till, "Exercise as Labour" - similar to the one performed by DTC testing consumers. 
paratus to take stock of ourselves. We need help from machines" ("Data-Driven" n.p.). For him those older techniques seem to have left us with incomplete pictures of the self, simply because we lacked the right tools to access the knowledge buried deep in our biology: "We don't have a pedometer in our feet, or a breathalyzer in our lungs, or a glucose monitor installed into our veins" (Wolf, "Data-Driven"). This is a discourse of deficiency in which the biological, material body on its own is framed as in need of technological intervention to reveal its secrets. The self, the (plastic) body are positioned as "deficient" and "improvable" (Abend and Fuchs 11), reinforcing the lack/lack rectified structure of self-help discourses. In this context the DIY approach might lose some of its power: the process of translation is often, especially in this commercialized, mainstream form of self-tracking, not done by the individual themselves. Instead, people have to rely on companies and machines to provide visualizations, representations of the masses of data the trackers collect. They gain access but might in the process relinquish some of their authority over the interpretation. From a critical point of view, thus, authority does not necessarily lie with the individual but with the machines that analyze the data or the companies that write the algorithms. Similar processes are discernable in DTC testing and through the new "experts of the soma" in body hacking as well.

As with many of the DIY techniques I discuss in this book, self-enhancement is also somewhat inherently connected to self-tracking - in its motivational matrix as well as in its cultural representation or promissory discourses. Measuring is often connected to some idea of improvement (Hoy 95). For example, "Know yourself to improve yourself" is used on the website of the famous fitness tracker company FitBit: "Fitbit motivates you to reach your health and fitness goals by tracking your activity, exercise, sleep, weight and more," they write (Fitbit, "Fitbit Official Site"). The basic framework seems to be that knowledge necessarily leads to improvement, or at least the realization of one's individual fitness goals. In her report on self-tracking, Hill writes that "[i]ts adherents believe that gadgets can help organize torrents of personal data that can help turn us all into happier, healthier, wealthier and more productive human beings" (n.p.). These goals, however, are either inherently subjective - such as happiness - or seem to conform to a neoliberal ideal of productivity. This is also reflected in other media accounts: Data, it is implied, have helped Dancy to change his self into a more "productive," efficient one, writes S. Murphy (n.p.). The goal of tracking, thus, always seems to be some sort of improvement of the self or the body. Self-tracking technology, Abend and Fuchs claim, is marketed as a "productive method of self-engineering," in which a malleable body can be shaped in reaction to the numbers collected from measuring its functions (11). Lupton discerns the entanglement of bodies, technologies and selves as an important dimension of the quantified self: technologies are "incorporated" into notions of embodiment and selfhood, extend and enhance them. As they have become digital, smaller, easier to wear, "it becomes less obvious where the body ends and technology begins" (Lupton, "Self-Tracking Cultures" 4-5). Devices have become extensions of ourselves. In effect, self-improvement as a goal of self-tracking means that the technologies and techniques that are used can and will have material effects on the body: changing its physique, its molecular make-up, its outside impression. What tracking promises, thus, is knowledge, control, optimization. 
Self-knowledge, authority, control, optimization - all these features of self-tracking cultures point to the strong emphasis on the self and personal experience in the self-tracking community (Lupton, Quantified Self 65-66). This is visible not only in the common name 'quantified self' or 'self-tracking' but also in the overall approach of individualized, personalized experiments and technologies. The self, on the first glance, seems to be the center and focal point. Some of the fears, doubts and reservations about the increasing quantification of life also hinge on this strong support for individualist thinking. Already in her subtitle S. Murphy points to the potential for negative side effects of the hyper-awareness fostered by self-tracking, a "lonely self-awareness." In the interview, Dancy admits that sometimes he feels lonely because behind all the data it is hard for him to connect to people: 'Being aware is not for the faint of heart,' he says. 'I think aware people live alone on a mountain" (n.p.). As a price for the connection to himself, it is implied, he has paid with connections to other people. Individualism trumps community. Apart from her fascination with Dancy's approach, S. Murphy finds this story terrifying as well: "Is a truly aware and connected life one that will make us isolated in a pool of our own data?" (n.p.). Murphy draws on common fears of depersonalization in the face of seemingly neutral, anonymous masses of digital data.

This hyper-individualization, on the other hand, seems to be in tension with the community-orientation of self-tracking enthusiasts. "Quantified Self" founder Gary Wolf insists that self-tracking is "not particularly individualistic," but that there is a "strong tendency...to share...and collaborate," to collectively create a new type of knowledge about human bodies and selves ("Know Thyself" n.p.). This sharing ethos is probably one of the most prevalent evocations of community in self-tracking (cf. N. Fox 137). Collecting data is often intimately linked to communicating data to others (Lupton, Quantified Self 111). Hill acknowledges the social nature of tracking as one of the reasons it intrigued her: "For another, I'm a social creature; I like to hang out with people online and offline, and sharing data is a great way to do it" (n.p.). This approach to social sharing is part of the network culture of the $21^{\text {st }}$ century: "Personal data are ideally suited to a social life of sharing. You might not always have something to say, but you always have a number to report," writes Wolf ("Data-Driven" n.p.). For example, FitBit evokes a community of "Fitbit users" one can join for tips, inspiration and more: "Now you can connect, share and compete when you want, with anyone you want" (Fitbit, "Why Fitbit"). Even if it sometimes appears "narcissistic," Wolf claims, self-tracking encourages new (types of) connections as well: "We leave traces of ourselves with our numbers, like insects putting down a trail of pheromones, and in times of crisis, these signals can lead us to others who share our concerns and care enough to help." ("DataDriven" n.p.). Numbers and data become a medium and means of communication.

Indeed, this connection to and care for others is highly visible in one of the affective dimensions of self-tracking, the tracking of moods and emotions to know and control them. Tools like "MoodPanda," a mobile and web app that allows you to track your mood on graphs and calendars and get support and advice from the "MoodPanda" community, or the similar "MoodScope" make it possible to effectively track feelings and emotions, thus quantifying more aspects of wellbeing and (psychological) health. But more than that, they consciously embed the tracking individual in a community that is created through the tracking itself, one that is meant to provide support and words of encour- 
agement in times of need - the data trail Wolf references. Critics Bietz and Hayes write that the "sociality" of self-tracking is one of its major benefits: Sharing data becomes an opportunity for motivation, social support and friendly competition (83). Instead of focusing on the individual, a multitude of communities are built around self-tracking, which always takes place in social situations (Neff and Nafus 3). It becomes clear that apart from being a "technology of the self," self-tracking is also a "technology of the social," not only based on monitoring the individual but on sharing self-data with others (Lemke, "Beyond Foucault"). Self-tracking, thus, is as much a 'do-it-yourself' as a 'doit-together' activity.

In the commercialized setting facilitated by the mainstream reliance on corporate hard- and software, personal data are gaining commercial value, constituting a new form of "biovalue" (Waldby). ${ }^{8}$ Nevertheless, there is also resistance against this consumerist, market-driven ideology that is to a degree also part of the communal aspect of self-tracking. According to Tamar Sharon some trackers enact their own autonomy by defining and tracking their own parameters and analytical categories or by hacking, dismantling and repurposing their tracking devices (109). In what Sharon calls "high-tech counterculturalism," digital technologies are used as "subversive tools of resistance:" Self-trackers often also tinker with their hard- and software or refuse to use proprietary software and data platforms. This type of resistance against surveillance and governance by corporations configures them "as active users, rather than passive consumers of technology" (Sharon 110-11). Self-trackers can also represent "a different way of knowing what data is, why it is important, who gets to interpret it, and to what ends:" This can according to Dawn Nafus and Jamie Sherman be a challenge to dominant big data practices and thus a form of "soft resistance" (as they call it) to the approaches of companies, firms, and data collectors (1785). This type of soft resistance, Abend and Fuchs summarize, means "that even within authoritative data structures, practices emerge that challenge the basic building blocks of the structure not by introducing alternatives but by playfully engaging with the technology in ways unintended by the industry and policy makers" (10). This is a tinker's approach not just to the technologies themselves but also the social and regulatory structures in which they are embedded. ${ }^{9}$ These resistant, counter-cultural moves by self-trackers - hacking and tinkering their way to individual devices and software - position them closer to the practices under consideration in the next case study.

\section{Case Study VIII: Real-Life Cyborgs - Grinders, Implants and Technological Immersion}

Tracking technology that is currently merged with the body from the outside, many scholars, futurists and pundits predict, might someday be integrated into the body.

Targeted advertising is one way of how personal data are used to generate commercial value. For example, some fertility and ovulation apps track data, which are then connected to Social Media profiles, allowing more targeted advisements for e.g., maternity and baby products (cf. Schechner and Secada for the Wall Street Journal). 
Majmudar et al., for example, write that future sensors "will be ingestible, implantable, or subcutaneous" (154). Similarly, Neil Harbisson, a self-described "cyborg," thinks that more invasive uses of technologies will become normalized: Once wearable technology is accepted and we have moved on to implants, merging human and technology will become completely normal (Weller). This type of integration not just with but into the body, of course, also takes hold of the biotechnological and cultural imaginary, elicits enthusiasm and worry as well as speculations about the future of the human species. This case study deals with people who are on the forefront of this development: 'Realworld' cyborgs, grinders and technological self-experimenters. Here I extend the idea of technological interaction with the body to a movement that seeks to integrate technology into the body in individualized projects of extension and enhancement.

My sources for this discussion are pop-cultural representations of biopunk modification, media articles as well as self-descriptions of grinder companies and communities. More specifically these include Dangerous Things. Custom Gadgetry for the Discerning Biohacker, a biohacking online shop and information source, and Grindhouse Wetware, a biohacking community in Pittsburgh, Pennsylvania. ${ }^{10}$ Founded by Amal Graafstra, himself a famous figure in the grinder scene, Dangerous Things has made it its mission to sell biohacking equipment and increase the safety of the products people put into their bodies. ${ }^{11}$ The technologies sold by Dangerous Things represent the two most common forms of integrating technology into the body, techniques that have become more and more widely used in the last years also due to their perceived low risks and easy implantation. They include RFID (Radio Frequency Identification) and NFC (Near Field Communication) transponders as well as biomagnets for sensing and lifting small objects, hearing and interacting with technology. Grindhouse Wetware is an open-source biohacking start-up, which has received media attention through a feature on "Cyborg America" for The Verge (Thonis). This group, as journalist Cameron Coward writes, was "born out of the biohack.me forums" (n.p.) and built on ideas of community and collaboration but also frustration with sweeping visions that were never carried to fruition. The devices they experiment with range from aesthetic updates of the body to potential medical applications: What they share, Grindhouse's members say, is that they are "built by cyborgs for cyborgs" (Thonis). One project is the "Northstar," an LED device that can be implanted under the skin. Coward describes it as "purely aesthetic," "a circuit board containing LEDs that can be lit up and seen through your skin" (n.p.), an aesthetic update that resembles bioluminescence (Melendez). ${ }^{12}$ Another of their projects

10 Other famous grinders include Lepth Anonym, who has made a name for herself through her reports on her own extreme body experiments - inserting magnets into her fingers with crude tools in her kitchen, for example - and Prof. Kevin Warwick, Professor of Cybernetics at the University of Reading, UK, who had a microchip implanted into his arm to control a robotic arm.

11 Amal Craafstra is one of the first people to use the technology on himself. He started his own 'journey' of augmentation in 2005 with an RFID tag: Taking inspiration from pet RFID tags, he found a programmable alternative that he had implanted in his hand to work as a door key card (Coward).

12 For Grindhouse Wetware the most likely application of such a technology is, for example, the potential of adding lights to tattoos. This device, it seems, is very much built by and for the body modification community. 
is a technological implant, "Circadia," that in their vision will be able to track medical data from inside the body. Their self-descriptions allow these niche practitioners to voice their own goals and concerns, while media reports on them and other 'cyborgs' might shine a more critical light on those practices and bring them into more mainstream discussions as well.

'Grinders' consider themselves a subgroup of the wider biohacker community: They are a small group of DIY biologists who use implants and synthetic materials to modify their bodies - essentially creating 'do-it-yourself Cyborgs.' On the first glance, grinding seems to be the most extreme form of biohacking, though on closer inspection it might actually be the result of following those other forms - body hacking, molecular modulation, DIY and DTC testing, tracking - to their logical conclusion. Bodies in the grinders' view become just another 'system' to be hacked, with the goal of transcending the natural body and human physical limitations. A definition given by the "biohack.me" Wiki, an online forum where the self-proclaimed "self-biohacker community" meets, defines grinders as follows:

Grinders are passionate individuals who believe the tools and knowledge of science belong to everyone. Crinders practice functional (sometimes extreme) body modification in an effort to improve the human condition. We hack ourselves with electronic hardware to extend and improve human capacities. Grinders believe in action, our bodies the experiment. (biohack.me Wiki, my italics)

A prerequisite for this movement is the ubiquity of bodily modifications: Tattoos and piercings have become mainstream, also brandings and scarring are not as unusual as they used to be. The body has become a playground of experimentation that individuals can form according to their liking. Combined with transhumanistic philosophies of chosen evolution and transformation through science as well as more affordable and available equipment and access to information in the $21^{\text {st }}$ century, people began to tinker around with technology and their bodies. Grinding, thus, is the result of a convergence of a DIY ethos, hacker culture, and the open science movement with increasing enthusiasm for bodily modifications.

Here, a DIY ideology is used for a more direct and invasive way of modifying the body by merging it with technology. In such a disputed field the 'DIY way' is often the only avenue towards realizing cyberpunk visions or testing out new, self-built technologies. While most people would assume human augmentation technologies would come from medical or technology companies, instead, "we're seeing augmentation begin in the basements of hackers and in the back rooms of piercing studios" (Coward). These informal spaces, we have learned, are typical settings for DIY biology and medicine. Fittingly, of course, grinding shares many features and topics with other practices of DIY biology and medicine: their hacker ethos and bottom-up approaches; democratization, open source and open science; sharing of and access to information; plasticity and malleability of the human body; self-directedness, self-experimentation and selfeducation; the urge to take control, action and frustration with the pace of technological innovation. Traces of all those can be found in self-descriptions and media representations. Since I have discussed most of them at length in previous case studies, here I want to focus on thematic complexes that build upon these foundational prin- 
ciples and values of grinding: the role of the cultural imaginary and structural overlap with older techniques of body modification, post- and transhumanist visions of extension/enhancement, hybridity and cyborg identity, as well as vulnerability.

Like few other examples in my discussion, this type of DIY biology is influenced by science fiction imaginaries and cyberpunk fantasies of human-machine hybrid bodies. The long-standing cultural preoccupation with technologically-transformed bodies sparked the imagination of people and made body-technology integration seem worth striving for. Grinders are makers that turn conceptual considerations and cultural fantasies into reality. These roots are reflected also in Cameron Coward's feature on body modification, augmentation and grinding: To the "average geek," the idea of augmentation is "at least somewhat intriguing" because most "have already been exposed to the idea through science fiction," he writes (n.p.). ${ }^{13}$ Despite the niche concentration of grinding as a practice, human augmentation is a widespread concern in mainstream cultural representations. A famous example of cyberpunk body modification is the character of Molly Millions in William Gibson's cyberpunk novel Neuromancer (1984). Molly Millions sports not just razor-blade prosthetic fingertips but also has artificially enhanced vision and a modified nervous system. This character exhibits body modification as an "endless process of customizing and upgrading," as Victoria Pitts argues (151). In this narrative universe, body modification is not dictated by biomedicine or cosmetic surgery experts but rather a "quotidian and populist project of survival and success"adaptations are a necessity in that high-tech, cyber-universe (Pitts 151).

A more contemporary example can be found in Netflix's techno dystopian science fiction show Black Mirror. In an episode titled "Arkangel" (2017) a mother decides to have the eponymous neural implant placed into her daughter's brain, allowing her to track her whereabouts, see through her eyes, monitor her stress level and medical status, or even block out potentially disturbing images and audio. Initially working successfully, it becomes a hindrance as her daughter grows up, letting her feel frustrated and cast out. A child psychologist diagnoses that the - by then controversial, soon-to-be-banned - Arkangel is responsible for her emotional immaturity and recommends turning it off. Black Mirror is known for taking on controversial or commonplace topics and technologies and thinking them to a dystopian conclusion, thereby presenting the viewer with a glimpse into possible futures and providing incentives for public discussion. Based on existing technology, in this episode it takes up ideas of implanting devices into the human body for the monitoring of medical data and psychological wellbeing, but also ties issues of surveillance and privacy into the discussion. These are only two examples that demonstrate the pervasiveness and long history of our cultural fascination with technological extension. The appeal of grinding is very much influenced by such science fiction visions of human bodies and their interconnection with technology. The strong influence of cultural representations on a very much material practice shows 
how entangled practices and representations are and once more emphasizes that we need to take (pop-)cultural representations into account as potentially transformative influences.

Orphan Black (2013-2017), a Canadian-American production running on Space in Canada, BBC America and Netflix, brings us closer to the real-world ideological basis of grinding (Manson and Fawcett). The series features "neolution" as an ideology and representation of contemporary cyberpunk artists and body hackers, espousing not just physical modifications of the human body, a tail for example, but also more sophisticated techniques such as cloning. ${ }^{14}$ "Neolutionism" is a fictional ideology or philosophical outlook that pursues human "directed evolution" and encourages scientific advancement through active modification (Orphan Black Tumblr). The goal is to improve the human condition, to carry forward human evolution through science. Such ideals position "neolutionism" as a fictive examination of transhumanism. Transhumanist and posthumanist ideologies often form the backdrop for cyberpunk body modifications. I do not want to go into a diversified discussion on the philosophy of transhumanism, but a brief introduction of some of its core concerns should suffice to provide some context for the underlying belief structures of much of the grinder community. Max More, a leading figure in contemporary transhumanism, describes acceleration of evolution, improvement of the human condition, enhancement, overcoming of (biological) limitations, and a focus on science and technology as some of the central themes, values and interests of this group of philosophies of life (3). "By thoughtfully, carefully, and yet boldly applying technology to ourselves, we can become something no longer accurately described as human - we can become posthuman," More writes (4). Post- or transhumanism, thus, can be summarized as an ideology that emphasizes progress, personal freedom and action, reason, science and technology - all with the end goal of directing human evolution into something 'more than human.' Dangerous Things adheres to this ideology when they proclaim that biohacking will lead the next phase of human evolution. The end goal of grinding, it is portrayed, is human enhancement, supplementing, augmenting the human body to become better, stronger, faster. Wohlsen includes such strategies in his account of Biopunk and also Mark O'Connell's To Be a Machine (2018) and Adam Piore's The Body Builders (2018) can serve as popular science representations of this type of transhumanist thinking that capture the cultural appeals and imaginaries as well as the skepticism and concerns.

The goal of human augmentation is to extend the human body and its capabilities into more than is possible today. For example, a mini feature video for The Verge called "Biohackers: A Journey Into Cyborg America" opens with the following words: "How much can I push the human, how much can I consciously evolve the human body to

14 The plot of Orphan Black revolves around a group of female "sister clones" who have found each other - sometimes by accident, sometimes through meticulous searches - and have now banded together to try to lead a self-determined life without the influence of the powerful biotech company that created them. A dominant theme is the creation and control of human life (through technological progress) as well as cloning and its effects on identity. In 2016, Orphan Black received the 68th Primetime Emmy Award for lead actress Tatiana Maslany, who plays all of the female clones featured in the series. It has also been nominated for and won a plethora of other Canadian and American awards. 
do more, to do it better, to do it faster, to do it stronger? I just wanna see how far I can push the human" (Thonis, 0:22-0:31). Conscious evolution and human enhancement are, from the beginning, positioned as the main imperative of biohacking, or grinding more specifically. One of the more common hacks in the grinder scene fills this idea of extending the human with life: Small magnets implanted into the finger "allow users to sense the presence of electromagnetic fields, to diagnose electrical problems like faulty wiring, and even to pull small metal objects like paper clips and bottle caps toward you, making you into something of a low-rent Magneto" (Melendez). ${ }^{15}$ They are a common, already realized way of giving the human body something like an extra sense, an extra ability.

To achieve this extension Graafstra and Dangerous Things count on the structural overlap and cooperation with body modification professionals and culture. They are positioned - and positioning themselves - as bringing order and safety into a widely unregulated scene. This safety is produced through training piercers and body modifiers in the safe and sterile handling of the equipment and by providing easy access to tested materials and technology. With body modification artists the grinder community has found collaborators that do not only share their interests but that as specialized "experts of the soma" (Rose, Politics of Life) can help to maximize safety and minimize the risks associated with such invasive procedures. But also ideologically, body modification culture and grinding follow similar goals and concerns. Cyberpunk body modification, Victoria Pitts claims, frames the body as a "limitless frontier for technological innovation" and exploration $(13,153)$. Body modifications for Pitts are embodiments of controversy and of social rebellion through the body (2), turning it into a (material) site of transgression and boundary blurring with symbolic potential. The cultural politics of body modification, thus, for many body modifiers is connected to authorship over their identity and symbolic meaning changes through alterations: they aim to change the significance their bodies have for them and others (Pitts 16-17). Changing one's physical appearance, here, becomes a means of shaping identities, of giving the body a new (cultural and political) significance as a site of fluid experimentation.

The new form of sensing promised by magnetic implants, Dangerous Things implies, allows us to attach a new label - a new identity one could argue - to people with implanted magnets: "magnetically augmented humans." This new possibility for self-identification turns the individual into a human-magnet hybrid, a low scale cyborg. Paul Atkinson claims that DIY, in the traditional sense, "allows a more individual aesthetic" than that produced by mass-production and consumption (1). This idea, I would argue, can be extended to DIY body modifications. They too allow for a more "individual" aesthetic and feel of the body. DIY frees grinders not just from dependence on corporations and consumerism but also allows for a more tailored, individualized approach that combines fun tinkering with the scientific exploration of new technologies. Modifying the body becomes a means of expressing identity and subversion as much as an

15 Note both the inclusion of "Magneto," part of the X-Men universe, as a cultural reference, as well as the description "low-rent" that points towards make-shift, improvised solutions. In fact, popcultural references are commonly used to describe the concept of cyborgs for broader masses of people, often by using Superhero characters, such as Tony Stark/Ironman (cf. Chapter 3.3). 
exhibition of technological progress. These techniques are also featured in Dark Net's "Upgrade" episode in the form of two biohackers who either already have a chip implanted or are getting one implanted during filming: The integration of technology into her body - "tech" becoming a "part of you" - is highly fascinating for one interviewee, a self-defined "tech nerd." During the filming of the episode, she has a chip implanted into her hand: For her the idea of connecting with another device is a "cool feeling" and she seems to accept the chip as part of her self (Jordan, "Upgrade"). The common discourse is that this type of technology can be seamlessly integrated not just into the body but also the sense of self as a technologically-augmented human, or technologyhuman hybrid.

Grinding here promises to make true long-standing visions of human-machine hybridity: A fleshy, real-life cyborg. Hybrids, Stevens writes, are "mixtures, crosses, or chimeras" that are disruptive in their transgression of social and cultural boundaries. Biotechnology, he argues, creates many kinds of crosses, between animal and human, animal and plant, living and nonliving (144-45). ${ }^{16}$ In the future, this type of hybridity might blend into the 'natural' body even more seamlessly. What is created is a cyborg, a "hybrid of machine and organism" as Donna Haraway defines it in her seminal text on cyberfeminism, "A Cyborg Manifesto" (5). In this text Haraway argues against the prevalent dualism in Western societies that construct binary (and from a feminist standpoint problematic) oppositions between for example body and mind, animal and machine, female and male, nature and culture. By blurring boundaries and borders the concept of the cyborg for her can be a way out of these dualisms (Haraway, "Cyborg Manifesto" 7,67). Referencing Haraway, Pitts summarizes that such a "cyborgian consciousness" "raises cyberfeminist hopes for a culture free of gender and further, for the deconstruction of all binary oppositions naturalized in the body" (159-60). In this cultural application of the concept of the cyborg, hybridity paves the way for an ongoing, fluctuating, and flexible play with bodies and identities. This playfulness and malleability find their materialization in the actual integration of technology and synthetic materials into the physical body itself. The result is a model of the cyborg that is both symbolic and real, material. A fleshy signifier of deconstruction and possibility, a science fiction figure brought to life and, in its transgressiveness, the embodiment of rebellion and non-conformity.

This image of the cyborg is often evoked in media representations. An example that has been widely reported on is Neil Harbisson, who had an antenna implanted into the base of his skull. Harbisson became famous in 2004 when he had his antennae implanted at 21 years of age. He is commonly described as a "real world cyborg" or alternatively the "world's first cyborg" in many media publications: Journalist Chris Weller, for example, writes that "[t]here is a name for people like Neil, who are part human and

16 An already realized example of this type of boundary defiance are medical devices and technologies that integrated into the patient's body take over vital functions, such as ventilators, kidney dialysis machines, or cardiac pacemakers, as well as prosthetic limbs that today can be interfaced with a patient's brain. These kinds of configurations "challenge the boundaries between body and machine and between animate and inanimate" (Stevens 144-45). 
part machine. Neil is a cyborg." (n.p.) ${ }^{17}$ Cyborgs, Weller describes, strive to alter their senses and bodies:

Generally speaking, cyborgs want to experience a heightened reality. Some put microchips under their skin to replace lock-and-key entries of their homes and cars. Others use brain stimulation to improve their focus. Neil, a British-born, Catalan-raised musician and activist who now lives in New York City, has decided his antenna known as an Eyeborg — will be his ticket to higher planes. (n.p.)

Neil Harbisson was born color-blind and had his antenna installed in order to transform color into sound waves that his brain can decode. "Neil still can't see color, but he says the antenna lets him hear and feel it" (Weller). Though his primary concern was to overcome a congenital limitation, he has 'upgraded' his antenna during the past decades: In 2013, for example, he augmented his antennae with Bluetooth connectivity that now lets him hear and feel color also via the Web. But Harbisson self-identifies as a cyborg as well, this identity is not only ascribed to him by the media. For him, there no longer is a distinction between his body and his technology, rather he feels like he is technology. His antenna is not only surgically sutured into his body but has become a naturalized sixth sense, an extension of his body.

A more experimental project by Grindhouse Wetware wants to move away from the sphere of playful augmentation and into the realm of medical application: The aim of their DIY micro-computer "Circadia" is to collect medical data inside the body. While not completely functional, during first tests for limited periods of time "Circadia" has successfully managed to collect and transmit temperature data. ${ }^{18}$ It is this implant that has drawn the most attention. Tim Cannon, a leading member of Grindhouse Wetware, had "Circadia" implanted into his forearm in late 2013 in Essen, Germany, by a wellknown body modification artist. "A lump of electronics the size of a deck of cards," Dan Sung writes in his article, this experiment successfully turned Cannon into "part human, part machine" (n.p.). Sung quotes Cannon as saying:

'I think a lot of people saw the size and the Frankenstein stitches. I understand why that makes people think what we've done is dismissible....This was not meant to be for the masses. This was for a test....We were just looking at if it was possible. What nobody

17 Neil Harbisson is not just a self-identified cyborg. He is also part of a cyborg movement lobbying for cyborg rights. One goal of this group is to start a conversation: "In 2010, Neil co-founded the Cyborg Foundation with fellow cyborg Moon Ribas. Moon has a seismic sensor implanted in her elbow that connects to online seismographs. Whenever an earthquake strikes around the world, a vibration lets Moon know. Neil admits the Cyborg Foundation's stated goals — of helping people become cyborgs, defending cyborg rights, and promoting cyborgism as an art movement - are still in their infancy." (Weller)

18 For the future, Grindhouse Wetware envisions a Circadia 2.o that can track more vital signs like blood glucose, heart rate, and oxygenation levels (Sung; Melendez). Sung describes this vision as a chemical tracker of the self, integrated into the body - the cyborg version of a wearable tracker. Melendez, however, points out that these potential uses might catapult Grindhouse Wetware out of the domain of self-experimentation and into that of FDA regulated medical devices, carrying with it problems that they want to circumvent with their open source and access for all ideology (Melendez). 
knew was whether things could be implanted safely, and used, and charged. Now that we've proved we can do it safely, we can do it smaller.' (n.p.)

In this passage Cannon refers back to one of the main aims of Grindhouse Wetware: To demonstrate the feasibility of technical implants. "Circadia," as Cameron Cowards acknowledges as well, shows that such implants are 'doable.' At the same time, this quote highlights the extreme approach to self-experimentation in the grinder community. It underlines what Tim Cannon stresses in the Verge feature on biohacking as well: Grinders like them take the pain, literally, now to make such technologies possible for the masses later.

Sung's account of Grindhouse Wetware is also, it seems, the most honest one. In it, Cannon opens up about the "psychological trauma" he experienced through wearing the "Circadia" for the 90-day test period. Even his own involvement in "bio-proofing" the device - keeping the electronics contained and making it as safe as possible - could not "stop the psychological trauma of carrying around a battery and circuits under your skin" (Sung). Sung quotes Cannon as experiencing "really bad panic attacks" for roughly a month after the implantation: "Every little tingle felt like the battery was bursting and I'd convince myself that the poison was entering my system. There were moments when I thought I'd crossed the line but I wouldn't be doing something bold if it wasn't a little scary." (n.p.) This perceived vulnerability, one could argue, comes from his new experience of cyborg embodiment, of carrying around a 'foreign' object in his body. ${ }^{19}$ What this representation makes clear it that technology might not be as "seamlessly" integrated into the body as grinders might wish. The "Frankenstein stitches" described by Cannon serve as a visual representation of this predicament.

\section{To Summarize}

Analogue self-inspection, these case studies have shown, today is no longer enough to satisfy human curiosity - and the demands for enhancement and optimization. These age-old techniques are supplemented with technologies that penetrate deeper into the body and provide instant feedback on the body's current status, potentially quite literally as the "Circadia" project has shown. As Lupton rightly detected, already the quantification of the self has enabled a further integration of technologies into bodies and selves, increasingly obliterating the boundaries between body and machine ("SelfTracking Cultures" 4-5). While self-trackers are usually worn on the body, grinding aims at embedding technology into the body, which takes the integration of technology one step further.

Technological implants that are used to restore form or function have been socially accepted and widely used, some of them already for decades. Cardiac pacemakers, implantable defibrillators, brain pacemakers, cochlea implants, retina chips, sophisticated

19 Even more "extreme" than the Circadia, Sung writes that Grindhouse Wetware also has abstract plans for an Open Heart Project, an optionally replaceable heart. This OpenHeart Project, might, in fact be the most obviously transhumanist project of Crindhouse Wetware because here the technological augmentation is not done out of aesthetic considerations or (individual) curiosity but as a means to enhance the self, extend its lifespan with mechanical devices without medical necessity. 
prosthetics, contraceptive implants and glucose measurement devices are mainstream forms of human-technological hybridity (cf. Lupton, "Self-Tracking Cultures" 4). They are nothing new or extreme but have become accepted as part of human 'nature' in a technologized society. As we can witness today, tracking technology is moving into a similar direction. Grinding takes this form of technological integration to a new level of 'outside' innovation, makeshift solutions, and open approaches. Their tools and technologies are not restoring function but simple aesthetic modifications, extra senses, or new means of tracking physiological data. As such, they seem to represent a new form of transgression between the boundaries of humans and machines, living and nonliving entities. They are perceived as more extreme, bloodier, more experimental, a niche movement of DIY enthusiasts and technical tinkerers.

What needs to be stressed is that as of now many of these techniques are still "in [their] infancy" (Coward): Biomagnets, chips and bioluminescence give just an inkling of future possibilities. Nonetheless, as other technologies have shown, widespread adoption of new technologies is often faster than we would imagine - think of smart phones, DTC genetic testing and fitness trackers as examples. What seems niche and extraordinary today might lay the foundation for future technologies and a more widespread acceptance of technological enhancements. The DIY techniques of today, thus, might through processes of normalization become commonplace applications of technology tomorrow. Self-tracking technologies worn on the body can be considered as a stepping stone towards this development. Cultural discussions will be necessary to decide to what extend such technologies truly are desirable and under which conditions.

Technological innovation will in the future make technological implants smaller and easier to do; their potential medical uses will attract the attention of the usual players in medical markets, start-ups and companies that aim to commercialize and profit of those early attempts. The small start-ups - more or less profit-oriented - that have already formed around the idea of implantable technology are likely to spread the phenomenon further by making the tools more easily accessible, thereby potentially positioning it closer to a commercialized DIY activity like the quantified self. But grinding is also a symbolic force. Much like the DIY EpiPencil and Aull's hemochromatosis test, grinding is a form of demonstration: that it is possible to undertake this type of intervention not just yourself but in general. Grinders as such also have a trailblazing role, they might pave the way for (commercialized) endeavors by delivering a proof of concept and an enthusiastic base. It is this symbolic force that makes grinding also political: It is a movement that advertises a radical freedom of choice about what to do with one's own body and biology, a "morphological freedom" (More 4) of form and function. 



\section{0 "The Human Need to Fiddle" - Tinkering with Technology}

Technology and tools - the material basis of DIY biology and medicine - are foundational for the spread of DIY techniques. But they are also intimately related to the overarching premises and ideology of DIY biology. What characterizes the use of tools and technology in DIY biology is their fluidity: Parts are recombined, 'misappropriated' in creative ways, shifting and changing in relation to the user's goals, a playful engagement with one's material surroundings, biology and body. "In light of the human need to fiddle," Wohlsen asks, "why would someone not want to [tinker with biology]?" (43) This playfulness, however, should not cloud the serious nature and transformative potential of those tools and technologies in action.

Tools have always been a primary condition for making: As Gelber has noted, the first wave of DIY as home improvements was facilitated and supported by the increasing availability and affordability of the necessary tools, machineries and materials to perform those improvements yourself: power-drills, fast-drying paint, pre-cut wallpaper, kits and instructions made it possible also for 'lay' users to take on projects in their home (cf. Gelber, Watson and Shove 73). Low-cost training and trial workshops to sell tools as well as classes to teach their use expanded the simple possession of tools with knowledge on how to correctly and safely use them, Gelber narrates. A similar development is visible in the area of DIY biology and medicine: Declining prices for electronics and biotechnology make the needed equipment more accessible and affordable, for DTC uses and DIY projects. Community workspaces, maker spaces and bio labs but also online tutorials and discussion boards have become spaces in which interested people are taught about their safe and correct uses. More than that: Inspired by DIY's third wave biohackers are beginning to redesign tools and create cheaper versions, instead of waiting for companies to provide tools redesigned with consumers in mind (Watson and Shove 73). Tools and technology, thus, become powerful agents of transformation, education and empowerment.

As such they have also drawn the attention of some of the scholars looking at the DIY movement. Morgan Meyer, for example, specifically focuses on the geography and spatiality of DIY bio in connection to its materiality: Through his focus on tools and ma- 
terials, he looks at the spaces where DIY bio is practiced as well as the boundaries that are constructed or demolished (Domesticating). Meyer argues that "the circulability, the affordability and the mutability of objects play a key role" in DIY biology by contributing to the recent emergence of a "citizen biotech-economy" ("Build Your Own" n.p.). These „citizen biotech-economies" are open, decentralized, collective, and accessible: They not only grant individuals material access to knowledge, objects, and infrastructure, but also imply a socio-political openness. By doing so, they try to foster "a material re-distribution, a democratisation, and an alternative to established, technoscience" (Meyer, "Build" n.p.). In these citizen biotech-economies tools and knowledge flow freely from institutionalized science towards citizen applications (and back as I would add), are distributed, used, rearranged, hacked, reconfigured, and recreated (Meyer, Domesticating 13). The case studies in this chapter exemplify parts of these alternative biotecheconomies.

Moreover, Meyer's concept already points to a core concern when it comes to the approach to tools and technologies in DIY biology and medicine: Playfulness, experimentation, curiosity about how they can be used differently - "tinkering with technology" as it is often described. For example, Wohlsen writes that the goal of DIY biology is to "increase the tinkerability' of biology" (24). "Tinkering," in this context, is used as a label to designate a certain approach, a certain type of inquiry. The nature and character of tinkering, according to Keulartz and van den Belt, can be inferred from a distinction made by French anthropologist Claude Lévi-Strauss in in La Pensée Sauvage (1962): Lévi-Strauss differentiates between "two modes of scientific thought," a "modern-day 'science of the abstract' based on 'domesticated' modes of thought" and a long-standing tradition of a "science of the concrete' based on 'wild' thought" (cited in Keulartz and van den Belt 6). "Whereas abstract science proceeds according to the methods of the engineer," Keulartz and van den Belt summarize, "the modus operandi of concrete science is that of the bricoleur or tinkerer" (6). Abstract science or engineering, for them, follows a plan, a goal according to which the materials used are designed. Tinkering or wild science, on the other hand, "works without a clear plan by making creative and resourceful use of whatever materials are at hand to produce new objects that possess some kind of unexpected functionality" (Keulartz and van den Belt 6). The epistemological approach of DIY biology, for Keulartz and van den Belt, is closer to Lévi-Strauss' concrete science of the bricoleur - practical, following a "hands-on imperative" taken from hacker culture with its emphasis on free access to tools and information, knowledge sharing and cheaper solutions (5-10). But DIY biology, as is visible in the citizen techno-economies, also uses tools available through and from institutionalized biology. What we have in DIY biology and medicine, thus, is a fruitful coexistence of both epistemic practices. Mann uses the designation "tinquiry" to capture the idea of "tinkering as inquiry" (29), an approach that also combines inquisitive engineering principles with the resourcefulness and playfulness of tinkering.

According to Delgado and Callen tinkering exhibits the "exploratory and fun dimension of hacking," it is characterized by openly trying things, sometimes repeatedly, in unconventional ways (189). Tinkering, therefore, is not distinct from hacking but rather a part of it. The exploratory and playful approach of tinkering is also visible in Heidi Ledford's notion of "creative work-arounds" (651). Ledford does not provide a defini- 
tion for these "work-arounds" or improvisations but she seems to try to capture the creative potential and unconventional nature of some of the technological solutions in DIY biology. Morgan Meyer takes Ledford's "creative workarounds" to denote "inventive ways to work without conventional and expensive material." These workarounds for him happen in two ways: first, the transformation and combination of objects in unusual ways, and second, the circumvention of established scientific institutions and industrial economies, for example when equipment is imitated or donated (Domesticating 14). Tinkering, thus, is characterized by creativity, ingenuity, resourcefulness, inquisitiveness. This approach is reflected in how tools are built, used, distributed and shared. But it also influences how technology is approached as a resource that individuals can shape and use according to their needs and wishes - with creativity and openness, not fixed but fluid.

Meyer argues that DIY biology unites the individual - the yourself - with the collectives around them (Domesticating 18). An individual's hack, the outcome of their tinkering, is seen as providing a benefit for the larger community. In this sense, DIY biology is not as much about individuals but more about their communities and collaborations. The 'yourself' is not a concrete individual using technologies on themselves, but an abstract idea of empowerment. DIY here is still often driven by curios minds or individuals, but more than that, it is a community approach and collaborative process. As I have argued in previous chapters and case studies, it is this social nature of DIY biology that brings us closer to its political nature. Delgado and Callen argue that hacking and DIY are technical as well as political demonstrations. They write: "What is hacked is not only a site that was inaccessible before (i.e. biology or electronics) but also the way of accessing it. By exposing themselves as easy and unsettled, hacks work as tangible demonstrations that 'you can also do it'. In doing so, such demonstrations are not only technical but also political." (189) DIY biology opens up not only different alternatives of doing something but also demonstrate that 'you' can do them too: DIY hacks "tangibly show that realities can be modified and that 'you' can do it" (Delgado and Callen 181). This is true for all the examples in the case studies under consideration here.

In this chapter I have chosen to focus on two different types of using, making and (re-)combining technology to create cheap, functional and practical solutions. Case study IX "Cheap and Functional," approaches DIY biology from a technical side, by looking at tools that are created in and for DIY labs. I look at the spaces and tools of the biohacking lab through their cultural representations and self-presentation. Case study $\mathrm{X}$ "Practical Solutions" then combines this view with projects that connect DIY tool-tinkering to medical uses, more specifically, two 'hacks' for DIY medical devices that were developed in the diabetes community - direct interventions into the body using a playful amalgamation of hacked, commercially available and self-made parts. This chapter thus shows real examples of how the DIY, maker, and hacker ethos is fruitfully employed in the area of biology and medicine. It nonetheless, of course, shares and exemplifies some of the core themes of DIY biology, such as access, open-source ideology, hacking and collaboration. 


\section{Case Study IX: Cheap and Functional - Making Tools for the Biohacking Lab}

Cheaper, easy to use and nonetheless highly accurate - those are the goals for many of the hacked solutions in DIY biology and medicine. This case study looks at its basics: the spaces and tools needed to perform biology yourself. More than mere examples of DIY possibilities, these reflect some of the most fundamental concerns of biohacking: materiality, domestication, simplicity, openness, access, as well as its social and political nature. In this case study I try to access these examples through their cultural representations, more precisely, the narrative that Marcus Wohlsen creates around them for his book Biopunk as well as some of their self-descriptions on the Web. Wohlsen's Biopunk, which I have mentioned a few times already, is one of the first comprehensive overviews of DIY biology in the non-fiction science sector. A trained journalist, Wohlsen has set out to describe the biohacking and DIY biology movement in its different facets - 'translating' practices and knowledge for his reader along the way. For this discussion, four of his chapters are particularly relevant because they look at ways in which technology is used, hacked and created. Already their titles can tell us a lot about how Wohlsen frames the biohacker's use of technology: Chapter 2, "Outsider Innovation," points to the distinction between institutionalized and 'domesticated' science as well as the hacker's position outside of the system; Chapter 4, "Make/Do," refers to the handson and creative approach to biology that is typical of tinkering; Chapter 5, "Field Testing," alludes to the possible scope of DIY innovations and with it questions of (global) access; Chapter 6, "Cheap is Life," indicates the goal of affordability - and thus access - as well as DIY's potential to save lives with its reconstructed tools for high-tech biology. At the heart of these chapters are specific tools designed and built by the DIY community as well as their makers.

Technology and tools for academics, journalists and practitioners alike have been crucial for the development of DIY biology and will continue to shape how it is practiced and by whom. Famous maker Chris Anderson includes an introduction to DIYbio in his description of the maker movement. What stands out is his pronounced focus on the cheaper, more accessible and modifiable lab tools produced by DIY biology: $\mathrm{He}$ argues that so far DIYbio has been "less about doing new science than about democratizing the tools of science" (C. Anderson 221-22). This democratization has taken different forms. On the one hand, we have the redistribution of technology that Meyer described: As a result of technological innovation and rapid price drops for biological technologies combined with the crisis of 'Big Bio' during the last recession, Delgado and Callen summarize, cheap and second-hand equipment has become more widely available on sites like eBay, making it easier and cheaper to set up laboratories (180). On the other hand, biohackers have taken on the challenge of creating alternative solutions to expensive technologies. Science journalist Erin Biba writes in her article for Wired that "thanks to the DIY revolution" and its open-source technologies like Arduino, biohackers can take a shot at "reverse engineering the big-budget tools" and then share their approaches: "Behind the scenes, engineers and science enthusiasts are teaming up to mod tools and technologies and then sell their inventions-or simply share tips on how to build them-to anyone interested," she writes (Biba, n.p.). Technologies are modified, recombined or copied and then brought into the community of biohackers. 
Wohlsen acknowledges this crucial role of tools for DIY biology as well. He references biohacker Mackenzie Cowell's believe that "the DIYbio approach will put the tools they need within reach." Simple, low-cost tools and techniques built by the amateur community, for Cowell, will lower the costs of setting up a lab tremendously (22). This focus on affordability and access is combined by Wohlsen with considerations of epistemic approaches - the often-referenced playfulness - as well as places such as garages and bars as new spheres where "science can happen." (23-24).

In fact, Wohlsen's second chapter opens with this very idea of new places and spaces for biotechnology, or more precisely, with the common image of two separate spheres: the lab and domestic spaces.

Labs are where science gets done. The home and the lab are separate places symbolizing separate realms of knowledge, separate practices, priorities, and precautions. This feeling holds especially true for molecular biology. Anything the size of a cell or smaller is out of scale with domestic life, where the only cell visible to the naked eye is the chicken egg in the refrigerator. (Wohlsen 18, my italics)

This passage alludes to the spaces in which science should be practiced but also the uneasiness about potentially invisible molecular agents in domesticated spaces like the home. His focus on symbolism is especially interesting since he references cultural norms and values, not just about what a 'lab' and a 'home' mean but also about different epistemic practices in science and outside of science. The home seems to represent 'wild science,' tinkering, while the lab stands for institutionalized science, engineering. This distinction between lab and home, I would argue, also corresponds to boundaries between home and work, private and public life that biology as a hobby - and not a profession - promises to derail. Very present as the narrator of his account, Wohlsen himself seems to be rather cautious about this new conflation of long-set cultural boundaries, as he demonstrates in the closing paragraph of this chapter:

Biohackers want to make the rest of us okay with the counterintuitive coming together of biotech and basements, of DNA and dinner tables. They not only believe the mass migration of biotech out of the lab and into the home should happen. They believe it will happen soon and that we all should pretty much not worry. But the sell may not be easy. (Wohlsen 27 , my italics)

He implicitly positions himself and the reader as apart from the biohacking scene, as cautious and critical voices against a "counterintuitive" foray of biohackers. Choosing this narrative frame - opening and closing the chapter with the same cautious objections - perpetuates the culturally entrenched uneasiness about the conflation of those 'separate' spheres.

Interestingly, his uneasiness about the mix-up of labs and homes does not seem to extend to community workshops, another primary site of biohacking and the main focus of Wohlsen's chapter. A community biohacking lab, we could presume, can also for Wohlsen become a new designated space of biology just like the industrial and research laboratories in Big Bio. "Outsider Innovation" is dominated by detailed descriptions of a maker space/community-based biology workshop called Sprout \& Company in a suburb of Boston, Massachusetts: 
The space holds everything a budding biohacker would crave. Two machines for Xeroxing DNA. A squat, steel, sterilizing autoclave, the biolab equivalent of a pressure cooker, complete with a vintage Mad Men-era analog pressure gauge sticking off the lid. A electric stirring rod. Tiny centrifuges. A hulking microscope, a rack of pipettes, a deep freezer to calm squirming microbes, and a bright-orange cabinet labeled FLAMMABLE LIQUID STORACE covered in magnetic poetry. On a high shelf sit bottles of the wet lab's wet stuff. Distilled water. Sodium thiosulfate, a chemistry lab standby. Contact lens solution. An old vodka bottle labeled with an indecipherable chemical formula. A bottle of agarose pellets to make the nutrient-rich gelatin broth that nourishes cells and allows them to multiply in petri dishes. (Wohlsen 21)

This paragraph familiarizes the reader with not just biohacking labs but science labs more generally. It does not only provide a detailed view into the lab with its tools, technologies, cabinets, bottles, pipettes and chemicals but also tries to 'translate' what these tools and chemicals do for a lay audience. Good examples are the "Xerox machine" for DNA, the pressure cooker, or the nutrient-rich, nourishing broth. At the same time, the scientific feel of the lab is interrupted with references to objects that are normally not expected in a lab - the magnetic poetry, contact lens solution, a repurposed vodka bottle. Whether or not tools like these are used in prototypical science labs, these objects do not conform to the standard, sterilized image of a laboratory and thus serve to underline the 'hacker' ethos of a DIY biology lab. They represent the blurring of boundaries that is so prevalent in DIY biology as well as the creative, make-shift solutions facilitated by its approach.

It is not surprising that how biohackers frame their research and approach their projects is also addressed by Wohlsen. An important figure for him is the "tinker." "Make/Do" addresses this underlying approach of DIY biology most explicitly. "In the hands of the most gifted practitioners," Wohlsen writes, "tinkering is an essential form of creativity. But it is a different brand of creativity, practiced in a different spirit, than the kind suggested by the romantic image of the lone artist or genius inventor trying to wrestle inspiration out of nothing." (40) Tinkering, Wohlsen argues, goes against individual technological innovation, against the tradition in the Western imagination of science and technology as a "heroic march of great (mostly) men and their work of genius" (40). ${ }^{1}$ For hackers, Wohlsen claims, it is the action that counts, the hack: "Their innovators are not heroes. They are mischief makers. And these tricksters do not sweat

This opening for the discussion of tinkering is interesting because even though he does embed them in a larger context, maybe even a group or community, Wohlsen is very much focused on the individuals behind the hacks. This focus, to some degree, evokes the discourse of individual genius typical of scientific knowledge production. Biohackers like Kay Aull, Tito Jankowsky or Meredith Patterson are described in much detail, from their outward appearance, to their educational background, hobbies and marital status. Of course, all these details serve to personalize the DIY biology movement, to give faces to an anonymous mass of, as it is sometimes perceived, potentially dangerous hackers and tinkerers. As such, this narrative approach creates sympathy and to a degree reflects the diversity of the movement. But it could also undermine the focus on community and collaboration, might mold its egalitarian, problem- and solution-oriented approach into standard narratives of scientific invention. 
their way to discovery. They tinker." (Wohlsen 41) From the beginning Wohlsen positions tinkering as creative, egalitarian, active, slightly mischievous.

The idea of "mischief makers" leads Wohlsen to the history of the figure of the tinker: This figure has a "rich, complicated history" in Irish and Scottish culture. For centuries, he writes, people at the bottom of the social hierarchy were termed a tinker. "As such, to tinker was to be a vagrant. Tinkering was not the work of an active, contributing member of society. The tinkerer was an outsider, not to be trusted." (41) In Irish literature, for example, the tinker is "a trickster, a rogue, the canny clown" (Wohlsen 41). From a cultural perspective tinkering thus for a long time was seen as rather negative. This negative history - and Wohlsen's use of it - however also emphasizes the re-appropriation of the term, the creative use of all the cultural baggage it carries with it, by turning its negative connotations on their head and reframing the outsider status as an emblem of progress. Today, Wohlsen writes, "[t]inkering has long since taken on a more generic sense of fiddling or tweaking, ...But it still retains the idea of work that is not really work. ...Tinkering is work you do for fun" (41). Following this reasoning, tinkering becomes a hobby, a work-like pastime, reminiscent of Gelber's description of DIY. Hackers "embrace" the playfulness of tinkering. Yet playing for them is not just entertainment, Wohlsen claims, but an "attitude toward innovation that champions gamesmanship, that prizes intellect applied with competitive vigor and flair" (42). Tinkering in Wohlsen's words becomes a form of material experimentation and an intellectual exercise at the same time. In line with the etymological considerations above, tinkering is thus turned into an epistemic approach and practice.

This intellectual and material experimentation can, according to Wohlsen, have truly transformative effects: "In the same way, the gifted tinkerer can rearrange the already existing engine parts or snippets of computer code in a way that creates something utterly new and potentially transformative" (42). Creative re-arrangements and workarounds characterize the tinkerer's (and the DIY biologists') approach to technology. ${ }^{2}$ The transformation, it seems, does not only take place on the material level of the tinkered object but potentially also on a larger scale. This type of inquiry, experimentation and production for Wohlsen does not only seem to be a particular ethos or approach but, he implies, also a "primal instinct" of the human: "If you believe that using tools is the essential feature that separates us from the other animals, then tinkering may be the most human urge of all - a truly primal instinct. If so, the impulse to biohack starts to seem self-explanatory." (Wohlsen 43) A similar stance is often visible in the maker movement where making is described by some as a fundamental human trait (Hatch). Biohacking becomes just another, self-explanatory way of feeding the human impulse to fiddle and create. In these lines, Wohlsen follows a somewhat naturalistic discourse that stands in contrast to the open, constructivist approach of DIY biology: Tinkering, this unusual, "ragged around the edges," creative approach to biotechnology is "source of much of the skepticism directed at outsider biology" because it runs against the "unshakable premise of wet lab work" - ordered, organized, sterile - that is believed to stave of the existential risks of biological experimentations for the research subjects and the wider community (Wohlsen 56). 
using tools, here becomes a primary marker of humanness, our biology just another material to use and abuse to feed our own instincts.

The creative use, modification and recombination of technology is one of the ways in which DIY biologists try to fulfil their vision of a democratized and domesticated science. "In DIY subcultures of all kinds," Wohlsen writes, "hacking the costs of materials, equipment, and labor is always a top priority" (60). Part of these efforts to hack the costs is the use of readily available or simpler tools to achieve the same purpose. A good example are common household "chemicals:" They are readily available and relatively affordable. That also means that the "raw materials of biotechnology are always just a supermarket away," as biohackers often observe (Wohlsen 47). Those simple tools, nonetheless, can provide profound and powerful insights:

Using a few household chemicals, anyone can break open a strawberry cell's walls and isolate its genetic core. At the end, you twirl the stringy stuff of life on the end of a chopstick. Suddenly DNA ceases to be an abstract concept, a sci-fi-tinged computeranimated demo of a double helix. Here it is, right in front of you: the physical crux of everything that lives. (Wohlsen 46)

A household chemical like dish soap can grant you a view into the material basis of life, turning DNA from an abstract concept into a concrete reality. But for many biohackers, using readily available materials simply is not enough: They want to make the high-tech equipment of Big Bio affordable and accessible for the masses.

One example is the "OpenPCR" project by biohackers Tito Jankowski and Josh Perfetto. PCR (Polymerase Chain Reaction) machines, or thermal cyclers, are an essential tool for amplifying DNA in a biological laboratory. Wohlsen describes these machines as a "photocopier" that "did for labs what Xerox machines did for offices" (Wohlsen 65). Basically, what a thermal cycler does is to heat up and cool down reagents and DNA in a specific order for specific periods of time, thereby allowing short DNA strands to be multiplied through polymerase chain reaction. This technique is used, for example, as a first step to map genomes, detect the presence of bacteria and viruses (through their DNA) or diagnose genetic disorders. Since PCR was invented, the machines that perform this work have, in Wohlsen's words, become "more advanced, more precise, more digital" but they also "cost a lot" (65). These high costs have for a long time prevented community laboratories and individuals from using the technology for their own purposes. But because PCR has become one of the most common techniques in molecular biology, DIY biologists have set out to construct their own versions of the machine.

Part of the "growing DIYbio and garage biotech movement" (Kickstarter, OpenPCR), Jankowski and Perfetto are two of them. Together, they wanted to create a low-cost, open alternative to this expensive but essential piece of equipment (Ledford 652). Wohlsen describes the OpenPCR as the "size of a toaster," made out of wood instead of plastic, with a digital display that "tracks the heating and cooling cycles" (Wohlsen 66). The "guts" of the machine are build using the open hardware system Arduino, and the "machine will tweet or text you when it's done" (Wohlsen 66). Available since 2011 OpenPCR is sold and distributed by Jankowski and Perfetto's company Chai Biotechnology. But its beginnings were much smaller. After building and improving their first prototypes, Jankowski and Perfetto looked to the crowd for enough capital 
to bring OpenPCR into production. In June 2010 they started a fundraising campaign on the famous platform Kickstarter. Their plan, they write, is to create an open-source design that anyone can build, that uses "off the shelf parts" and is easy to maintain (Kickstarter, OpenPCR). Their campaign was immensely successful. In about 10 days they had reached their initial goal of $\$ 6,000$ and until July they had doubled their goal. With more than $\$ 12,000$ the project was officially funded (Kickstarter, OpenPCR). Their promise of openness and access seems to have won over the Kickstarter crowd and the DIY biology movement. This approach of openness is also emphasized on their website. OpenPCR, they write, is "100\% Open Source;" hardware, software and protocols are openly available (Chai Biotechnologies, "OpenPCR - the \$499"). Customers can either buy a DIY kit or download the "IKEA-like" open-source instructions. Open source here becomes a facilitator of access but also a means of engaging a community, of making products better by involving the masses.

Openness, for biohackers, equals access. On the OpenPCR website, the machine is described as "A Thermocycler for Anywhere" that due to its low cost and small size "makes PCR accessible to anyone" whether in a small lab on a budget or in the field (Chai Biotechnologies, "OpenPCR - the \$499"). Fittingly, their aim is to "democratize access to molecular diagnostics:" They try to make these "fundamental technologies" "universally accessible, without exorbitant costs or intellectual property issues." This universal access, for them, would allow people all over the world to use PCR to address "challenging global issues," for example "point-of-care medical diagnostics in developing countries" (Chai Biotechnologies, "OpenPCR About"). Their vision is a "world where molecular diagnostics are routinely used by all and do not require specialized knowledge" (Chai Biotechnologies, "OpenPCR About"). Here their DIY biology background takes effect: What counts is the use of the technologies, the action, the hack, and not the credentials of the user. ${ }^{3}$

Their motivation, therefore, aligns with broader concerns of distributive justice and globalized access: Who has access to technologies and who is disenfranchised from their use (and benefits)? Wohlsen takes up this concern as well: He tells the story of Guido Núñez-Mujica, a Venezuelan biohacker. Núñez-Mujica is one of the developers of the LavaAmp, another tool used to replicate DNA. The LavaAmp is a small thermal cycler, a metal box that heats up, cools down, and heats up again making PCR possible. The

The Bento lab is a similar, somewhat younger and more commercialized alternative: Funded through Kickstarter as well (raising thrice their goal in 36 hours), the Bento lab is described as a genetics and biology lab for everybody, whose basic version can now be bought for roughly $£ 1,500$. What the producers of Bento criticize is that despite being "fundamental technologies of life" that "concern all of us," genetics and molecular biology are still the domain of a "small number of experts" with access to laboratories and knowledge. Bento is a personal DNA analysis lab, that includes a centrifuge, PCR thermocycler and gel visualization, through which they want to make molecular biology inclusive and accessible, enabling "professionals and non-professionals to engage with genetics in an open and responsible way" (Bento Bioworks). Easy to use and travel with it is meant as a tool for "hands-on" genetics that can be used in different settings: The user reports they provide range from molecular biology as a hobby, to hands-on teaching in schools and colleges, to citizen science efforts. Cenetics, it is their wish and promise, can with the Bento lab be experienced everywhere by everyone (Bento Bioworks). 
LavaAmp, according to Wohlsen, is the progress of science boiled down into a simple yet remarkable piece of equipment that will let scientists around the world manipulate DNA "at least about as easily" as in a lab (Wohlsen 55). Núñez-Mujica is himself a trained biologist who wanted to create a new form of test for a disease prevalent in his home region based on the genetic signature of the parasite itself. This test, ideally, would be portable, simple, and off-the-grid, so that it could work as a "rapid response kit" for underserved regions. "Núñez-Mujica," Wohlsen writes, "was inspired by the desire to invent more options for those like himself with access to less" (50-51). Structural factors are important here:

His stake in making the tools and techniques of biotech accessible to more people is more personal than that of most of his peers in the United States and Europe. Biohackers in the developed world are typically outsiders by choice. Companies and schools have the tools, but do-it-yourself biologists are willing to trade that access for the freedom to do what they want the way they want to do it. (Wohlsen 50, my italics)

Núñez-Mujica's university lab, according to Wohlsen, has a lower budget than a typical American school lab. In the face of high prices, they necessarily have to be inventive. Wohlsen's evocation of "outsiders by choice" is particularly interesting because it highlights how biohackers in the "developed world" are privileged despite their own abandonment of this privilege. In other countries, it is implied, biohacking becomes a necessary means of 'doing' biology at all. It is here that DIY biology could leave its biggest mark, by fulfilling the hopes of biohackers like Núñez-Mujica to "make the tools and techniques of biotech truly available to everyone" no matter where in the world they are (Wohlsen 52).

What becomes apparent in Wohlsen's discussions of tools and technology in the biohacking movement is its political nature and potential. DIY biology is portrayed as a bottom-up project with potentially transformative impact. Wohlsen writes:

Will these playful projects pile up until they reach a mystical critical mass when some alchemy of innovation transforms fun into serious and important work? In Patterson's ideal world, the fruits of creativity will sprout from below rather than fall from above. Moreover, if innovation emerges from the ground up, then perhaps its benefits will come more quickly to those at the bottom. (Wohlsen 48)

This bottom-up approach, Wohlsen communicates, has the potential to include more people into the innovations and transformations that DIY biology brings with it, especially those usually disenfranchised from scientific innovation. In this quote, the bottom-up approach ties into the concern with distributive justice, globalized access and participation in technological change. DIY biology, once more, becomes not necessarily an individualized preoccupation but a social concern.

Tito Jankowski, according to Wohlsen, argues that today we are faced with a new context, in which people want to contribute to biotech in set-ups that differ from typical lab situations, such as those in garages, community labs, or high schools (Wohlsen 68). What is at stake, here, are also questions of literacy and biological education. Erin Biba writes that "people inside the biohacker movement," when asked about where it will have the most impact, "talk about education-being able to do genetics in classrooms" 
(n.p.). Jankowski, Wohlsen writes, "hopes to introduce young people to the tools and techniques of biotech in a way that makes gene tweaking as much as part of everyday technology as texting." This educational focus, the aim of increasing the biological and scientific literacy of a broader mass of people, will allow them to "recognize and contribute to" "biotechnology's great promise" (Wohlsen 68). The question of literacy, taken up again and again by Wohlsen and his exemplary biohackers, is one of taking part in social, cultural and political discussions about the (future) uses of biotechnologies. Hands-on approaches as they are promoted in DIY biology and medicine are perceived to be better facilitators of this type of literacy than typical forms of translation, mediation and popularization.

In addition, Wohlsen emphasizes that DIY biology can also be political for the self. "Jankowski," he writes, "hopes that tools like his will help more people overcome the feeling of disconnection they have from their DNA" (68). The alienation from an abstract idea of DNA, according to Jankowski, should and can be overcome through an active engagement with its underlying concepts and ideas. Similarly, Wohlsen writes, Mackenzie Cowell thinks that through DIY biology, "outsiders" can be "shown that they can have fun with science, can play with these tools and ideas that are treated with such gravitas." By facilitating a more playful approach to science, Cowell believes, "they may begin to feel closer to themselves as they unlock the mysteries of their own organic being" (Wohlsen 25). Here, DIY biology - in its different forms of concrete engagement with biological materials - is connected to hope for more self-knowledge and a closer relation to (one's own) biology. The politics of DIY biology begin to intervene in the personal just as much as the personal can become political due to its embeddedness in social and cultural structures.

\section{Case Study X: Practical Solutions - Open Medical Devices and the Diabetes Community}

The ideology of open access, collaboration and practical solutions that we can witness in DIY biology's use of laboratory tools and technologies also extends to medical problems and assistive devices. In this case study I look at citizen science and maker approaches to medical-technologies that promise individualized solutions. ${ }^{4}$ Also here making and doit-yourself have led to fruitful co-operations, creative approaches and transformative 
experiences. More than in the examples in case study IX, when medical technologies are concerned the hack materializes in and on human bodies, changing and shaping lives and experiences of embodiment. A special focus is on the Diabetes community who have been actively involved in creating DIY solutions for problems that commercial providers did not (yet) address and have, according to Dana Lewis, been "experimenting and selfmodifying diabetes devices and technologies for many decades" to achieve better quality of life results (D. Lewis, "History").

DIY solutions to medical devices are, of course, set in the broader context of the (American) health care system, especially the move towards personalization and domestication of monitoring and therapy previously conducted in health care institutions (N. Fox 138) and the turn towards more patient involvement and responsibility. Today, mobile devices allow "consumers to diagnose and treat their own medical conditions without the presence of a health professional" (Greene 306). Such practices of "DIY Medicine" are getting more common through cheaper and portable technologies such as sensors and genetic tests (Delgado and Callen 179). But more than that, medicine has also become a sphere for hacker involvement, for DIY solutions to unavailable or expensive technologies - as some of the examples in previous case studies have already demonstrated. Delgado and Callen argue that DIY medical makers and hacks are a response to a perceived precariousness and vulnerability of the body in times of reduced state-facilitated health care. As such they can be considered as political demonstrations that reveal the precariousness embedded in the system and at the same time evidence technical ways of coping with it (Delgado and Callen 179). The geographical distribution of medical-technology hacks underlines this proposition: Developing alternatives to established health care practices through DIY practices is more common in the maker/hacker sphere in the US than in European groups (Keulartz and van den Belt 17). In a system of collective health care and welfare DIY initiatives are not as important as in an individualized, market-driven system that routinely excludes people from its services. What is more, these technologies are also often positioned as good alternatives for people in countries that lack comprehensive, accessible and affordable health care around the globe.

DIY medicine, however, is not a completely new phenomenon: Historically, the first DIYers in medicine were clinicians and medical professionals, a generation of post-war "clinician-engineers" that "tinkered" with medical technologies with the aim of making them cheaper and more widely available. According to Greene, "do it" for them meant "the ability of savvy amateurs to build and reengineer their own medical devices" and "yourself" was limited to physicians (Greene 306-07). Today, in contrast, DIY in the context of health care technologies often does not mean a "productive act of building" but "the consumptive act of buying" proprietary software, technology and applications, Greene maintains. "Doing it" equals becoming actively involved in your own diagnosis and treatment, bypassing the doctor, and "yourself" indicates the "patient-consumer" (306-307). While this type of modern DIY does fulfill its "liberatory promise" by freeing the patient from dependence on doctors or the clinic, it also creates a new type of dependence on applications, technologies and the companies that produce them (Greene 306-07). 
Between these two, there is room for creativity and experimentation. As Greene argues as well, DIY today can take on another meaning, one between professional engineering and individual consumption: "an expanding community of device hackers and self-experimenting amateurs now use their considerable technological literacy to circumvent physicians, manufacturers, and regulators alike" (307). This third way of DIY medicine - the tinkerers and hackers' way - often includes "strategies for retooling their own devices" such as prostheses or insulin pumps (307). For Greene, despite the risks associated with individuals potentially altering lifesaving medical devices themselves, this is a "powerfully egalitarian" form of DIY medicine that emphasizes the users' role in the production of medical technologies (307). In medicine, this type of 'tinkering' with existing technologies to serve a personalized agenda is one of the main forms of DIY interventions. People use existing tools and reshape them into an individualized solution, subversively reengineering them in the process.

Nick J. Fox takes on personal health technologies (PHTs) as one form of DIY medicine. He explores the assemblages of bodies, technologies, and relations around PHT use in order to assess their micropolitics, also in relation to different stakeholders and future developments..$^{5}$ One of his examples is the micropolitics of insulin pumps: A "fully integrated blood glucose monitor/insulin pump" does, he writes, "liberate" people with diabetes from "time-consuming and complex self-management," but it also "fundamentally" changes the relationship between disease, patient and professionals.

Responsibility for self-management is removed from users, replacing an 'expert patient' (Shaw and Baker, 2004) with sophisticated understanding of his or her disease and its management with a 'dumb patient' who merely has to wear the device and follow any instructions it provides to the user (e.g. to inject an additional insulin bolus if blood glucose goes too high). (N. Fox 142)

Instead of fostering a collaborative connection between patient and professional, it preserves an outdated one, based on passive patients and active medical specialists, manufactures and devices (N. Fox 141-42). In contrast, the practices under discussion in this case study undermine his criticism of "dumb," uninformed users reliant on experts and technology: Instead, they demand a patient that is actively shaping the process, makes themselves familiar with the technology and its potential shortcomings. I will demonstrate that the DIY approach catapults the patient into the role of designer and producer, a specialist on their individual devices. Their disease is not passively managed by machines and professionals but actively intervened in by the patient.

The Nightscout Project, a free and open-source solution developed for children and adults with Type 1 Diabetes (TiD), is a prominent example of how commercialized solutions are extended, by patient-specialists, to serve their own needs. It is a DIY modification of a commercially available continuous glucose monitor (CGM), a small device that people with diabetes can use to measure their glucose level - a clinically useful type of

Fox focuses on the examples of four different PHTs with differing objectives, from biomedical to independent uses: Blood pressure monitors, Fitbits and self-tracking technologies, insulin pumps as well as implantable cardioverter defibrillators for the monitoring and management of heart arrhythmias (N. J. Fox 139-40). 
self-tracking. CGMs work subcutaneously with needle sensors, can be worn for different periods of time and measure the blood glucose level at pre-set intervals of one to five minutes. Older systems had one big disadvantage: They did not allow users to directly view their data or access them remotely. The Nightscout Project "allows real time access to CGM data via a personal website, smartwatch viewers, or apps and widgets available for smartphones" (The Nightscout Project). Especially parents of TiD children were looking for more reassurance in monitoring their children's data while they were out of their immediate reach. The goal of the project, thus, was to "allow remote monitoring of a TiD's glucose level using existing monitoring devices" (The Nightscout Project). Nightscout, or alternatively called "CGM in the Cloud," was developed by volunteers to enable those parents "to view their child's glucose level while the child was at school, at daycare, playing sports, at a sleepover, or while traveling overseas" (The Nightscout Foundation, "Our Mission"). ${ }^{6}$ How does the "CGM in the Cloud" work? It is a simple and creative recombination of off-the shelf technologies. Basically, the CGM continuous to measure the glucose level and sends it to the respective receiver or monitor as usual. Users now attach a device to this monitor, that transmits the readings to the internet, where you can then view the data with other web-connected devices. Today, this type of system can be set up for a range of commercially available CGMs.

The development of the first DIY modification is also often narrativized in media representations of the Nightscout Project and its community of users: The entry point, most of the time, are the worries of parents with TiD children, more specifically those of Evan Costik's parents. At four years of age Evan was diagnosed with TiD and his whole family had to adjust to this new situation. His parents had to monitor their son's glucose levels closely, including regular nighttime needle pricks and tests, Mike Bradley writes for Wired. Soon after, they switched to a CGM, easing his parents' worries a bit, at least while he was in their immediate reach. "But children, it turns out, sometimes leave the house," narrates Bradley. And in this case, their display unit leaves with them, meaning that parents now have to rely on others to check the devices and care for their child (Bradley). John Costik's first experience of dropping Evan off at daycare, Bradley continues the narrative, was the starting point of his hacked solution: the "lack of information" was so "nerve-wracking" that the programmer started creating a DIY solution, a way to coax the CGM to display the data on the display unit but also send it to a cloud, where he could access them from his computer. "He started tinkering" (Bradley). The result of this tinkering was an "Android app that let him monitor Evan's blood sugar on a phone" - a solution, Bradley writes, "that seems so simple it's crazy it didn't already exist." What he created was an obvious, simple solution that made use of already existing technologies by recombining them in a fruitful way. The narrative of Nightscout's proliferation, as it is most often told, continues as follows: John Costik tweeted a screen

6 A short note on sources: The Nightscout Foundation has been created out of the Nightscout Project Facebook Group, both implemented in 2014. As a non-profit, it can for example generate donations to fund further research and simplify discussions with other agencies, companies or the FDA. The scope of the foundation is slightly broader, including closed-loop systems like the OpenAPS as well. Both homepages, however, can work as information sources for those interested in this DIY technology hack. 
shot of his hack on Twitter and, Bradley notes, started a "mini-revolution" among all the parents who had wanted similar access to their childrens' data. As Smith writes in his article "A Do-it-Yourself Revolution in Diabetes Care" for the New York Times - note the similar vocabulary - by sharing this photo Costik not only caught the attention of a "legion of parents" willing to create similar homemade solutions, but together they "set in motion a remarkable, egalitarian push for improved technology to manage diabetes care, rarely seen in the top-down world of medical devices" (P. Smith). The Nightscout Project as a bottom-up, need-based solution is portrayed as a potentially transformative, political act.

The "legions" of parents willing to try such a system points to one of the central features of the Nightscout Project: its community orientation. As the Nightscout homepage explains: "There is a community on Facebook with members who are parents of children with $\mathrm{T} 1$, partners of $\mathrm{T} 1$, and people with $\mathrm{T} 1$ from all experience levels who will help you get up to speed on understanding and installing this tool." (The Nightscout Project) As of April 2021, this "CGM in the Cloud" Facebook group had more than 35,000 members. The website emphasizes again and again that the development of Nightscout, its many new applications and the support for "newcomers" is the result of a large and active community of volunteers (The Nightscout Project). This community, it seems, is also made visible by the Nightscout Foundation: Their welcome page features a collage of alternating pictures of (presumable) users and activists of all ages - younger and older kids, adults, male, female - often with their technologies, mostly in the form of smart watches displaying data. The impression one gets is not just one of a lively, active and diverse community but also one of empowerment, of allowing individuals to participate in everyday life with less restrictions (The Nightscout Foundation, "Home"). The Nightscout Foundation is very much community-centered in the narratives they create but also in the potential they see: They believe that taping "into the capabilities and drive of the CGM in the Cloud community and the larger diabetes community" can lead to better approaches in diabetes care (The Nightscout Foundation, "Who are We"). Doit-yourself, also in this case, equals Do-It-Together.

The capabilities of the diabetes community, of course, have already shown their power in the Nightscout system itself. As the website emphasizes, it was „[c]reated for and by people with Type One Diabetes" (The Nightscout Project). They do not only have higher stakes in the development of safer products, but possess a more intimate knowledge about what they and their children actually need. As Smith writes: "Now, as consumer gadgets weave themselves ever more tightly into everyday life, patients and their families are finding homespun solutions to problems medical-device manufacturers originally did not address." (P. Smith) These work-arounds are centered on the patient and their needs not on what a corporation offers and the FDA approves - even though, according to Smith, the movement of user-driven innovation has impelled the FDA to approve new models faster. ${ }^{7}$ The personal, here, is tightly integrated into the political, even the commercial: Personal needs are translated into commercial development, activism is used as a blueprint for new devices centered on the patients themselves. The 
Nightscout Foundation, not surprisingly, is also trying to foster and promote such political engagements: They see a lot of potential for truly "patient-centered research" in this highly active community. They are tapping into this potential by partnering with patients and caregivers from the community to "create opportunities for participatory design, citizen science, and open science diabetes research" (The Nightscout Foundation, "Who are We"). It is this high degree of participation that debunks Fox's thesis on CGMs and integrated systems as fostering uninformed patients reliant on their system. In the case of DIY solutions, the opposite is the case: patients and their caregivers become experts on the technologies, their potential failings and the management of the disease.

To emphasize their idea that you do not have to wait to access the tools that could help you the Nightscout Project adopted the moniker "\#wearenotwaiting" in 2013 (The Nightscout Project; The Nightscout Foundation, "Home"). This moniker also implies a certain impatience with the commercial providers of monitoring devices, FDA regulations and institutional structures that hindered or delayed the availability of commercial tools with similar capabilities. But the DIY approach does not only come from an unwillingness to wait "for the FDA's blessing," as Bradley writes, or a bottom-up "push" for improved technologies through "homemade solutions," as Smith maintains - though both are important and transformative facets of the political nature of Nightscout's approach. Rather, it is the extension of the hacker ideal, the maker ideal towards medical technology. Not for nothing Bradley and Smith both call it a "hack." The Nightscout Foundation, they say themselves, is "unique" among diabetes communities for advocating for a "maker movement approach to TiD healthcare" and "citizen science" efforts that use open-source development methods to "create products and projects that offer immediate impact" (FAQs). They follow a broader ideal that perfectly aligns with the self-made ethos of DIY biology and medicine. However, this DIY approach, the Nightscout website explains, should be carefully considered as people need to be "comfortable configuring and installing [their] own system" (The Nightscout Project). In such a DIY approach, they rightly say, there is no warranty or insurance of any kind: "The quality and performance of the project is with you if you choose to use it." The self is responsible for how well the system works and how they use it.

Another development in diabetes care also goes back to the work done by the Nightscout community: the OpenAPS. Basically, the OpenAPS is one already functional application of a long-standing dream - an artificial, bionic pancreas, a "closed loop." On the technological side, such visions of an artificial pancreas combine a CGM and an insulin pump: Based on the data of the CGM and an algorithm the individual is given adjusted doses of insulin through the pump, which promises to reduce extreme blood sugar highs and lows through automatization. Bradley describes this vision of an "artificial, or bionic, pancreas" that controls "blood sugars automatically, just like

8 In fact, a rather long disclaimer on their website makes this abundantly clear: They stress that the system can fail, that the code is provided for educational use only, that it is a volunteer-based system without formal support, that the system lacks password protection and data security, and that Nightscout code or information should not be used for medical decision making. 
the biological one is supposed to do" as the "most tantalizing" idea in diabetes care. ${ }^{9}$ Here, you might notice, we move closer to the realm of technological integration into the human body, a technological hybridity that carries the grinder's vision of cyborg bodies into medical applications.

The OpenAPS is a "basic closed loop APS technology" that is "widely available to anyone with compatible medical devices who is willing to build their own system" (Dana Lewis \& the \#OpenAPS community, "OpenAPS.org"). On the OpenAPS website, it is described as an "open and transparent" effort to make "safe and effective basic Artificial Pancreas System (APS) technology" available to more people, more quickly than commercialized and FDA-approved products. Fittingly, the OpenAPS has taken over the moniker \#wearenotwaiting from Nightscout. The OpenAPS community, they write, have already created a "safety-focused reference design, a toolset, and an open-source reference implementation that can be used by any individual - or any medical device manufacturer." The goal is to "reduce the burden of Type 1 diabetes" through open-source and free technology but also to accelerate the innovation and testing process of more advanced APS systems (Dana Lewis \& the \#OpenAPS community, "OpenAPS.org"). The OpenAPS is a DIY solution, that as of February 3, 2021, according to their website, more than " $(n=1) * 2,211+$ individuals around the world" have implemented in some form or other (Dana Lewis \& the \#OpenAPS community, "Outcomes"). The first DIY closed loop was implemented in December 2014 - a time during which commercial applications of this type were still predicted to be months or years away (Dana Lewis \& the \#OpenAPS community, "OpenAPS.org”).

This uncertainty about when and how such systems will be commercially available is also one of the main motivations for building an OpenAPS. On the homepage of the OpenAPS we can read: "Individuals who build OpenAPS setups have decided that \#WeAreNotWaiting to be able to automatically adjust basal rates to safely keep BGs in range and reduce the burden of living with Type 1 diabetes." (Dana Lewis \& the \#OpenAPS community, "OpenAPS.org") A need is addressed by these technologies for which individuals do not yet find commercialized and approved answers; it is thus not just fun tinkering but has a serious context. This is also transported in many of the media representations of the OpenAPS community and its creation. Often, they position the ability and freedom to choose whether patients want to wait or use a DIY solution as one of the main effects of the OpenAPS (D. Lewis, "Experience"; L. Martin). The DIY approach, here, gives people options they previously did not have, it is a demonstration of feasibility as much as a tool of empowerment.

The creation and dissemination of the OpenAPS is intimately connected to one name: Dana Lewis. In the media representations she is the voice and face the community as well. The first closed loop was hers. In an article for the Guardian Lewis writes about her self-care as a TiD:

9 Commercially available diabetes care is beginning to close this loop as well. Some devices are already available that combine a CCM with a pump so that we are currently moving closer to FDA approved, commercial closed loop systems. 
This process became a little easier when I got an insulin pump, and later a continuous glucose monitor (CCM). The pump continuously infuses insulin into my body, and the CCM sensor can report my blood sugar every five minutes. At night, I relied on the CCM's built-in alarm to wake me if my glucose passed the threshold that required immediate action; but though I tried several CCMs, the alarms were never loud enough, and they couldn't be turned up or changed. I talked to manufacturers, but nothing improved. Once I went to college and lived alone, this became a bigger problem; I was increasingly afraid of going to sleep at night. (D. Lewis, "Experience")

Though she could manage her diabetes fairly well, especially nights were a time during which her CGM did not give her the certainty she desired. Living on her own, the lack of louder alarms increasingly became a source of worry that the commercial devices could not obliterate. While the alarms work for most people, "clearly [she was] not one of those people" (L. Martin). To her frustration, her desire for personalization could not be met by commercialized devices. But one day, the narrative commonly goes, she stumbled over Costik's tweet about his CGM hack. Using his code, she and her partner not only set up a system that would communicate the data to her phone and wake her with louder alarms in case of a low but also informed her partner if she slept through the alarm (D. Lewis, "Experience"). They also created an algorithm that would predict highs and lows and send alarms to her phone or smartwatch. These measures fended of her fear of going to sleep, she writes, but in this "open loop" system, she was still the one who had to take action. Working with others in the open-source diabetes community, she started to try to close the loop. As Martin writes:

With help from Costik, and an active open source community sharing information on how to access the functionality of CCMs and insulin pumps, Lewis was able to really get started. Within a year she and her boyfriend (now husband) Scott Leibrand moved from creating customizable alarms to creating algorithms to read data from her CCM and send the correct commands to her insulin pump to make proactive dosage adjustments, closing the loop and creating her first DIY pancreas. (L. Martin)

Closing the loop, thus, once more was a community effort in which knowledge about diabetes care met professional knowledge of programming and coding.

Having implemented her own loop, Lewis decided that she wanted to stick to the spirit of the open-source community and provide knowledge on how to do it online:

Having created something that changed and improved my own life, and having benefited from others' open-source work, I wanted to share our work so other people could use it. This is why we created OpenAPS - the Open Artificial Pancreas System project: to make the code, design and documentation available to others for free, so they can build a "pancreas" of their own. (D. Lewis, "Experience")

The OpenAPS is emblematic of what open-source design can do: Not only did Lewis take knowledge that was available open source and develop it further with the help of other people, but she also fed the new device and knowledge that was created back into the community for others to use, extend and improve. This system went from "being a personal project to being an open source, community-focused effort" (L. Martin). A growing 
community of interested volunteers and people with closed loops, Lewis writes, is continuing to improve the code and system - in their spare time, for free (D. Lewis, "Experience"). This community also provides advice to those who want to implement their own loops. The internet as a medium to share, connect and inform is particularly important here. Fittingly, for Morgan Meyer the internet plays an important part in the emerging alternative biotech-economies because it is a platform on which tools and equipment can be sold and bought, knowledge and instructions about finding and building alternative tools shared, and people connected to other interested people (Domesticating 13). My source, the website, is a case in point: Here people find open reference designs and documentations, mailing lists with troubleshooting guides, chat rooms where "OpenAPS users hang out" and can help answer questions (Dana Lewis \& the \#OpenAPS community, "OpenAPS.org"). Websites, forums, blogs, videos, according to Meyer, "are part and parcel of the material infrastructure that allows for the circulation of knowledge to take place, for collectives of do-it-yourself biologists to emerge, and for various kinds of boundaries to be overcome" (Domesticating 18). The new technologies of the Web 2.0 do not only allow for a proliferation of those ideas and hacks, but they also actively create and reinforce community.

Still, even more than in the Nightscout Project, the automatization of a natural biological function raises concerns about its safety. Without human interference, the imagined scenarios go, the system has to be watertight and failsafe for it to be considered secure. The OpenAPS takes these concerns into account. The OpenAPS, they claim, is built for safety. If the system collapses, parts break or are lost, the other ones can still be used independently and manually. The OpenAPS, they write, "follows the same basic diabetes math that a person would do to calculate a needed adjustment to their BG - but it is automated and precise" (Dana Lewis \& the \#OpenAPS community, "OpenAPS.org"). More than safe, they seem to argue, its automatization and precision makes it less vulnerable to the flaws inherent in human actions. Lewis writes that for her "having a computer make adjustments while I sleep is far safer than trying groggily to make decisions overnight" (D. Lewis, "Experience"). The system, we can read, is also designed to work smoother than humans managing their diabetes manually: instead of infrequent large adjustments, it "makes small adjustments every five minutes" (D. Lewis, "Experience"). This is supposed to minimize spikes and balance glucose levels.

Some of the concern about such open solutions arises from their emphasis on 'doit-yourself.' People tinkering around with medical devices are in the popular imagination taking part in potentially life-threatening and dangerous endeavors - the circumvention of regulatory bodies and standard protocols. That this type of DIY is actually a highly productive enterprise is often forgotten. "100\% DIY," apart from the "existing diabetes devices," this system allows people more options for personalization and customization, because they choose which technologies and algorithms they use (L. Martin). This individualization, however, comes with a cost: As with the Nightscout setup it is the individual themselves that is responsible for how well, safe and efficient the system works. The website makes it quite clear that it has to be a self-made solution: "You'll have to build your implementation yourself (no one can/will do it for you!)" (Dana Lewis \& the \#OpenAPS community, "OpenAPS.org”). You can get help from the community, the message goes, but you will need to do it yourself. In order to do so, of course, 
you need to know what you are doing - a fact that helps to make the system safer. The individual is a still required as an active manager of their diabetes: "Overall, it's important to understand that OpenAPS is not a 'set and forget' type of system. You'll still be actively managing your diabetes and doing basic self-care as you were before - this includes everything from meal boluses, checking BG and calibrating the CGM, changing out pump sites, etc." (Dana Lewis \& the \#OpenAPS community, "OpenAPS.org") The OpenAPS is an upgrade of previous self-care regimes, one that can even out glucose levels and provide an extra security net. It does, however, not replace self-responsibility. This, once more, counters Fox's concern of integrated systems creating "dumb" patients reliant on technology and algorithms. The OpenAPS becomes a means of empowering people to take their diabetes care into their own hands.

As medical technologies, such hacks leave traces on their user's body. Similar to the implants in the previous chapter, these examples merge the human body and technology: Blood glucose monitors and insulin pumps are integrated into the body, allowing for a closer monitoring of their functions. These technologies inscribe themselves on the bodies that use them, leave their material traces as much as they have social consequences. Lewis communicates some of these physical changes: After her first night with the closed loop system, she "was blown away by how much better [she] felt" (D. Lewis, "Experience"). An automized biochemical adjustment had managed to level out fluctuations in her blood glucose levels that she previously might only have noted subconsciously - it leaves its molecular traces on the body. This effect was so staggering for her that she decided to use the system permanently, thus integrating the technology (temporarily) into her body and possibly conception of self. The open diabetes community has done (self-reported) studies on the health outcomes of DIY closed loops, with reports ranging from better sleep, less mental burden, higher quality of life to less visits to school nurses (D. Lewis, "History").

But more than that OpenAPS is also an advocacy project of sorts. OpenAPS frames itself as a communication starter, a mediator between different actors in a complex web of commercial, legal and personal interests:

We are working to demonstrate the need of the community to have closed loop devices; demonstrate the safety of basic closed loop systems and efficacy compared to 'standard of care' without closed loops; and help break down communication barriers between the FDA, these companies, and the community that needs the devices widely available as quickly as possible. (Dana Lewis \& the \#OpenAPS community, "OpenAPS.org")

They advocate for collaboration between the DIY users and commercial providers, so that in the end all people with Diabetes will profit from their learnings and experience (D. Lewis, "History"). Even if the goal of commercial availability is reached, Lewis explains to Martin, that would not be the end of OpenAPS because also those systems might not be perfect or accessible for all (L. Martin). While currently an individual project of people with access to technology, the OpenAPS might in the future become a means of granting access to closed systems also to those who lack the financial means to buy them commercially or lack access to a healthcare system that makes them available and reimburses the costs. Here, we come closer to the political potential of DIY medicine. 


\section{To Summarize}

Both case studies represent truly bottom-up approaches to biotechnology and medical devices - the tools and technologies of DIY biology and medicine. Tools in biohacking labs are a precondition for DIY biology that has to be met in order to make biology accessible and affordable. These tinkered, collaborative, creative approaches do not only represent a different epistemic approach to biology and medicine, but they also work to enable the participation of all those who are interested in the future of biotechnology. What they aim for, on a broader scale, is the inclusion of those in the processes and practices of cutting-edge science that are usually disenfranchised from them: people in low- and middle-income countries, people with lack of access to comprehensive and affordable health care. As such, it is no wonder that a global perspective comes into play. Cheaper equipment comes with the promise of participation no matter where in the world (citizen) scientists practice their research or satisfy their curiosity. Open medical technologies, similarly, promise access to better management of diseases and technologies that truly meet the users' needs. It is here that we see these DIY solutions interact more closely with individual bodies, leaving their traces and changing experiences of embodiment. Both case studies make biology and medicine deeply personal, stress the individual nature, their hope for a more personalized engagement with and understanding of biology, a familiarization with their own bodies as organic beings on the most minute level. These technologies turn patients and users into more knowledgeable experts on their own bodies: The DIY approach is based on a comprehensive understanding of both the technology and the biological processes to be intervened in.

If we consider the OpenAPS system and the assessment that it is not a 'set and forget' type of system but rather a support system for conventional techniques of diabetes selfcare, this preoccupation with medical, biological, technological literacy becomes obvious. Especially the Nightscout Project and the OpenAPS demand a thorough biological and technical literacy for their implementation: In order to set up the system, people need to know how diabetes affects the body, how it is managed, how the technologies used to manage it function and how they can merge different technologies into a working system. Dana Lewis acknowledges this barrier to access as well: "DIY is unfortunately not $100 \%$ accessible to all," she writes in a commentary for the Journal of Diabetes Science and Technology ("History"). This need for extensive literacy, we should note, in effect stands in marked contrast to their aim - excluding people from participation instead of including them. This question of literacy also ties into concerns about safety: Greene writes that technological literacy determines "whether doing it yourself is ultimately liberating or dangerous." The question for him is how we decide who is "literate" enough to assess the risks of self-experimentation. This problem, however, is also inherent in other technologies, for example expensive wearables with promissory marketing (307-08).

Literacy, as the examples in this chapter have shown, can also depend on the collaborations individuals pursue - information and knowledge can be shared, DIYers educated by other DIYers. In such a context, literacy is something that can be produced in community rather than being an individualist (in)ability. In these new alliances people work together on an equal basis, they are part of a community instead of dependent on commercial providers or governmental regulations. As such these examples could also 
be considered as extensive educational efforts. Similarly, many of the tools developed for the DIY laboratory also have an explicit educational focus. They aim at making biology and biotechnology an ordinary part of everyday life, to demystify the processes of biology. Their focus on activity, on hands-on learning, aims to foster the ability to not just understand but actually 'do' biology. 


\section{PART IV: REFLECTIONS}





\title{
11 Discussing DIY
}

\author{
"As biohackers it is our responsibility to act as \\ emissaries of science, creating new scientists \\ out of everyone we meet." (Patterson n.p.)
}

Through hacking, making and tinkering, DIY biologists have necessarily become political actors - as Meredith Patterson writes in her Biopunk Manifesto (n.p.). ${ }^{1}$ Their practices draw on and question larger sociocultural concerns, make political claims and perpetuate ideologies of power, participation and division. Some DIY techniques create deep uncertainties about how individual bodies should and can be managed, about their social implications, about problematic consequences for the personal and public spheres of life. From a feminist technoscience view, the individualist rhetoric of 'do-it-yourself' might distort how these practices are part of hierarchies, systems of power and ideologies, that they are not just individual concerns but deeply political - to follow Pitts, they create privileges and constraints, shape the access to and control of biotechnologies, are embedded in political and social contexts and systems of power (cf. 160). In a similar way that numbers in the Quantified Self movement are not neutral but embedded in social dynamics and power relations, DIY activities are political in that they can also be "constructive of subjects, identities and communities" (Lupton, Quantified Self 96). As cultural phenomenon and socio-political activism DIY biology creates spaces and possibilities for wider public dialogue about biotechnology and biology, their technologies as well as their broader implications for individuals and society (Stevens 355 ).

In his expansive analysis of biohacking, Alessandro Delfanti summarizes the discourses that have been spun around DIY biology as follows:

DIY biology has been described in terms of open and peer knowledge production, a danger to public health, a co-optation phenomenon, a democratic (or apocalyptic) change in the relationship between experts and non-experts, an ethical dilemma and an experiment in public engagement with science. (Biohackers 113) 
While this list is certainly not all-encompassing, all of these descriptions are visible also in my case studies: DIY is variously a vehicle for knowledge production and dissemination, a danger to personal and public safety, a form of commercialization, an impetus for change and transformation, and a type of self-directed engagement with science and biology. These different configurations are owed to the diversity of practices, approaches, methods in DIY biology and medicine that is reflected in their representations in popular culture. This chapter aims to take a step back from this diversity and arrive at some of the broader consequences of and contested issues around DIY biology and medicine that point to its political, social, cultural and material effects. By doing so, it connects back to the theoretical discussions in the beginning as well as the cultural context of DIY. Here, we also come to some of the core questions of the humanities power, discrimination, class, justice, embodiment, agency. In this reflection I want to move across scales - from the individual to the collective and institutional, from the molecular to the structural and systematic.

On the basis of the case studies, I delineated concerns and public discussions that weave themselves through the narratives and should be part of a critical assessment of DIY biology and medicine's culture: a deep concern with questions of access, literacy and democratization (11.1); a trend towards commercialization and possible exploitation of its promises (11.2); concern about the security and safety of DIY techniques on a personal as well as public level (11.2); a shift in power relations and expertise (11.3); new forms of vulnerability and uncertainty (11.3); a belief in individualist solutions that undermine community principles and create new forms of differentiation but (conversely) also a tendency towards collaboration, sharing and community-formation (11.4). While not all of these areas of problematization are relevant for all DIY techniques discussed in Part III, many do share them to differing degrees. These concerns are also the scaffolding for the four parts of this discussion. ${ }^{2}$ They are fleshed out with questions for further debate: What is DIY biology and medicine's potential for social change? How can DIY shape access to equipment, knowledge, power? How will the increasing consumerization of modern society affect DIY? What are the effects of DIY on the definitions of lay people and experts? Can individualism and collectivity be brought into a fruitful combination? Because DIY biology and medicine is an emergent phenomenon, none of the questions can today be answered with certainty: Rather, we need to raise them and keep them in mind to assess future developments and shape discussions.

\section{1 "Power to the People" - Access and Participation, Literacy and Democracy}

"Scientific literacy is necessary for a functioning society in the modern age. Scientific literacy is not science education. A person educated in science can understand science; a scientifically literate person can "do* science." (Patterson n.p.)

2 The division into thematic complexes that I have arrived at might seem artificial because there is definite overlap between the different parts. I have, however, tried to set these concerns into dialogue with each other to map out some of the main problematizations. 
Is or can DIY biology and medicine be a political movement? As the case studies in the previous section have shown, all of the buzzwords in the subtitle - participation, access, literacy, democracy - are in one way or another part of the discourses and practices around different DIY techniques in biology and medicine. They are either part of their ideological scaffoldings (participation, access) or an important outcome (literacy, democracy). The goals of widespread scientific literacy, of greater citizen involvement in bioscience, of more equality in the production and use of biological knowledge and tools, is one that points to the deep political roots of this discussion. But is this 'DIY democracy' more than a rhetorical configuration or promissory narrative?

We increasingly rely on practical applications of science and technology for the management of our everyday life. Scientific solutions to everyday problems, also of the body and self, tantalize us with their claims of truth value, neutrality, objectivity, rigorous testing and experimentation. Scientific knowledge is perceived as "better than other forms of knowledge" because it is "external, neutral and separate from society," a means of accessing "truth" (Erickson 216). Scientism gives science, medicine and technology privileged access and authority over the body. DIY biology and medicine can be considered as one expression of this scientism in contemporary culture. Science, as the case studies have shown, is the backbone of the hacking of biological materials, a prerequisite to engage with one's own biology and that of one's environment. DIY, it seems, is positioned as a way for every individual to not only rely on science, have one's life shaped by science, but to actively engage with it. However, science itself, its modes and spaces of knowledge production are also often perceived as elite, separate from dayto-day life - it is this perceived demarcation that makes it indispensable to tear down barriers, both real and imagined.

\section{Knowledge as a Publicly-Owned Good}

DIY biology draws on scientism and science's authority, but it also - crucially - questions this core assertion that 'science' can only be practiced by people with specialized training in specialized rooms and environments. Traditionally, research in universities and industry laboratories requires college education, credentials, and long, rigorous training, thus essentially excluding large portions of society from partaking in it. In contrast, DIY biology claims that science can be done by everyone, to differing degrees, that engagement with science is a necessity, even a fundamental right in a technoscientific society. To do so, of course, people need to be granted or rather claim access. Affordable equipment, like the OpenPCR machine or Bento lab, allows DIY biology groups to conduct outreach programs that provide the lay public with hands-on training and education. Access literally and figuratively 'opens' the doors of science for a broader public and enables them to participate. As the case studies have shown, this participation in DIY biology and medicine can take different forms: from partaking in discussions around the use of biotechnologies online or in person to the actual use of them. The important part is that more people are enabled to understand and use the tools of science. DIY biology, also for Kay Aull, is a way to democratize science and give people access to their own biology "in the most direct way possible" (Wolinsky 684). This 
access, we have seen, is framed as both ideological and spatial: Access to information and knowledge as well as access to tools, resources and infrastructure.

With the notion of 'access' we are also entering a territory that can be regarded as inherently American, as it draws on fundamental values of equality and self-sufficiency, the ideal of liberty. If we take the Civil Rights Movement, Feminist activism or the Disability Rights Movement as examples, similar questions of access have been widely debated in American culture for the last century: access to rights, access to places, access to privileges, on an abstract level, have inflected these debates as well. ${ }^{3}$ In these movements, disenfranchised parts of the population struggle(d) for social participation, political representation and shifting scales of power. Similarly, the type of access and participation facilitated by DIY biology can also, following Meyer, be regarded as a form of "counter-power" in the hands of citizens that allows them to shape the use of biomedical technologies (Delfanti, Biohackers 125; Meyer, "Build"). In the struggle over access and power, DIY biology and medicine, thus is part of a historical continuity of political movements.

But we can also take this idea of access further and critically question if access in this case can and should be granted to everyone. This is not just a moral or ideological but also a practical question: Should everyone be able to fiddle with biological materials and what would be the consequences? Is the DIY approach even aiming at access for all? Are not the complexity and costs naturally disenfranchising some? I am thinking, for example, about people with (learning) disabilities, those with lower incomes or underprivileged class backgrounds, less schooling or access to education, questions of differentiation I will come to later. The current make-up of the DIYbio movement - people with high levels of education, often white, middle-class (men) with enough disposable income (cf. Jen 127) - is a case in point. What the discussion of access, in its essence, goes back to are questions of authority that as of today are deeply discriminatory: Who has the right to use or 'do' science? Are there conditions for doing science? Who should be involved in the discussions around the use of science? Who has and who should have the authority to decide? Christopher Kelty argues that much of the anxiety around "outlaw biology" or DIY biology hinges on the demarcations of who a 'real' scientist is, who gets to practice science, who is allowed to tinker, and who regulates scientific activity. The main struggle, for him, is one of legitimacy: Who gets to decide the future of biology? What counts as knowledge? Who can create knowledge? (3) Citing widely-known biohacker Rob Carlson, journalist Sigal Samuel includes this question of legitimacy into his article: Calling its practitioners "hackers," for Carlson, is a way of delegitimizing them, categorizing them as Other. For Samuel, this is "actually a bigger societal question: Who's qualified to do anything?" ("How Biohackers" n.p.). voting rights and equal access to desegregated spaces, physical access and accommodations for people with disabilities. All these hinge on the question of whether or not disenfranchised populations are allowed - by the majority - to participate equally in public life and to gain access to spaces previously closed from their view. In DIY biology the majority is disenfranchised from participation and access, while a small minority of people (those in science industries) have the privileges the former claim for themselves. 
DIY biologists would argue for a "popular ownership" (Steinberg 54) of techniques and knowledge. While Deborah Steinberg uses the phrase "popular ownership" only in passing, I want to elaborate a bit more on this idea because I think it is very much applicable here: Popular ownership of knowledge in my understanding means that this knowledge is in the hands of the general public not experts or specialists - that everyone has the right to have access to that knowledge. Knowledge becomes a public property, with all the rights and responsibilities that come along with that. "Popularly" or publicly owned knowledge is not restricted to experts, and more so, its use is not restricted to experts. This popular ownership is necessary because it allows a broader public to engage in the discussions also around the practical application of biological knowledge: As Henk ten Have argues, before knowledge is applied goals need to identified, benefits and harms balanced, and norms and values that need to be respected should be delineated (295). This is a process that needs to be public and held among a diverse group because most biological applications today might have broader consequences for human life and embodiment, the social fabric and solidarity. Thus, as publicly-owned goods the tools, technologies and knowledge of science are reframed as a concern for all of us. They should therefore not be proprietary or locked away but visible, usable, debatable. Science and its applications are turned into a public good, something that has to be discussed in the public sphere. But they also seem to promise a form of utopia that as of now seems far from reach. Does DIY really increase participation on such a large scale?

Despite some promising beginnings, this question, as of today, cannot be answered with certainty. Rather, the polarization of public discourse visible at the current moment in time - the debates around 'fake news,' the opinion making and targeted manipulations, conspiracy theories and mistrust of scientific advice - should raise the question whether broad-scale access would be used, also by anti-experts, for fraudulent manipulation or exploitation, whether the legitimacy and standards of science would be disfigured beyond recognition. Can openness and access foster trust in science, or will they contribute to more skepticism? Boyd argues that when scientific discoveries and debates play out in the spotlight, they demonstrate the fallibility of the scientific process, visible for example during the Covid-19 pandemic or in climate change research. The realization that an "institution" previously believed to deliver facts and certainty is uncertain itself, debates findings and revokes theories, might impact the public's trust in this institution. However, Boyd's argument relies heavily on (paternalistic) distinctions between experts and non-experts that risk increasing skepticism of science. Access to similar information, he argues, risks making individuals feel "as though they ought to be participants in dialogue as opposed to mere recipients of imperatives" (12). I would claim that especially in times of biological crises such a dialogical form of communication might be better suited to foster trust: Making people feel heard and acknowledged and making the scientific process transparent and accessible - as is the goal of DIY

The idea of property of course is also ambiguous because today biological materials or biological knowledge can actually be the 'property' of companies through patenting. This, of course, contradicts my conception of biological knowledge as a public property and is something I see deeply critical. 
biology groups. Still, what this assumption hinges on is the willingness of people to take part, their interest and concern: A generalized rejection of scientific findings will most likely not be overcome by simply opening the doors of science. That would require collaborative efforts across all sectors of society and institutionalized possibilities for dialogue and exchange.

What we can discern, however, is the inherent potential of this shift towards access and participation. The dominant principles and ideologies of openness, access, and creativity turn DIY into a new form of generating knowledge based on make-shift solutions, creativity, tinkering, fun, trial and error and characterized by hands-on, active and engaged education. What is created is an "open, bottom-up, collaborative model" (C. Anderson 78). Even though his argument, of course, is much broader, some of J. Dewey's insights into political inquiry (1927) can prompt a further discussion here. According to Dewey questions of common concern should in a democracy be deliberated on by exactly such a process of experimentation and exploratory action: a creative, imaginative, collective approach to "knowledge-making" that for him is "intrinsic" to political action, with the potential to break with old, problematic orders in favor of "unforeseen possibilities" (cited by Delgado and Callen 181). Following this line of thinking, DIY biology and medicine, through their transformation of how knowledge is created and by whom, also open up new potentials for political action and social transformation. They can break not just with the established order of scientific inquiry but potentially create even wider shifts in how subjects understand themselves as political actors. DIY biology and medicine can create a new way of approaching science, not as something apart from your own experience but as potentially an integral - and recognized as such - part of your daily life..$^{5}$ In this "epistemology of participation" (Delfanti, "Users and Peers" 3 ) science becomes something that you can do yourself, in whatever way you want to: on your own body, in citizen science projects, through experimentation or creative engagement with biological materials. This new epistemological approach can be transformative on many levels. For one, it can change how science is practiced: The generation of knowledge still takes place with standard scientific tools and protocols but on a more personal scale or together with new actors. It can also create new types of knowledge, for example by using the potential of the much-cited 'crowd,' the wisdom of the many, to find new solutions and new approaches to problems. ${ }^{6}$ In this shift from individualist, reclusive research enterprises to collaborative and open efforts new, creative ways might be found to address some of the most pressing questions of today, such as cures for human diseases, climate change or species diversity. Most fundamentally, however, it can

As I have written above, of course science is an integral part of our everyday lives - be it in the news reports we read, the medical decision we make or simply the technologies we use. Its influence, however, often does not seem to be recognized as substantial. DIY techniques have the potential to much more deeply embed not just the end-products of scientific innovation but also the process of their construction into everyday activities.

6 Crowd-sourced knowledge production has made its way into popular culture as well: Based on her column for the New York Times, in the Netflix show Diagnosis Dr. Lisa Sanders uses the 'wisdom of the many' to find diagnoses for mysterious and rare medical conditions. After publishing patient stories in her column, she asks readers from around the world to contribute their own experiences - be it healthcare professionals, researchers or patients with similar symptoms. 
transform who is involved in these practices by shifting the site of scientific innovation from elitist institutions to a much broader base.

\section{"It's Not Magic"}

What is needed as a first step for this broader base to make use of the new-found means of participation and knowledge creation is a more detailed understanding of biology and science. Here, DIY biology and medicine, once more, comes into play as a form of translation, mediation and demystification. Meyer argues that with DIY biology the laboratory can "potentially become a place of 'cultural translation' between amateurs and professionals" (Domesticating 4). In DIY biology and medicine knowledge is communicated to a broader base of people in a language that also lay people understand. The question is how and where should such translation ideally take place? If access to biological knowledge is necessary for political participation, does the state or society have an obligation to facilitate these types of translation practices by, for example, making them part of school curricula? According to Delfanti, educational and social goals are very important for DIY biologists, as can be seen in the "increasing inclusion of DIYbio practices in museum exhibitions, science education projects, and outreach activities for young people" ("Do-It-Yourself" n.p.).

As many of the DIY techniques in the case studies have shown, engaging with your own biology is also a form of demystification: abstract ideas and concepts become concrete, tangible and personal. For example, the NightScout Project and the OpenAPS require people with TiD to actively engage with not just their diabetes - and the molecular workings of the body in response to low or high blood sugars - but also with the technology they routinely use to monitor their bodies. It is a fundamentally personal project. Similarly, (DTC) genetic or molecular testing ideally requires individuals to become more familiar with the concept of DNA as inherently personal information and the molecular pathways and interactions they are testing for. As such, DIY biology and medicine can become a means of familiarization with science and biology more generally and one's own biological make-up more specifically. This demystification means that science no longer is imagined as a closed off, magical, mysterious, untouchable realm. Instead, biology, according to Delgado and Callen, is reimagined as "mundane," no longer holy or exclusive but an inherent part of being human (184). "It's not magic. It's chemistry,"' as Wohlsen quotes Kay Aull (14-15).

DIY biology and medicine can thus, as an overarching goal, contribute to a higher scientific and biological literacy. Literacy comes up again and again, in academic discussions as well as in the discourses around DIY biology: scientific literacy, data literacy, biological literacy, and technical literacy are evoked to describe citizen's ability to understand, evaluate and partake in the discussions around techno-scientific innovations. Knowledge and education, it seems, are positioned as some of the most basic things needed to become responsible actors and political subjects. In the last lines of Wohlsen's Biopunk, he arrives at literacy as an important result of biohacking practices as well: 
The opening of science, if done well and communicated effectively, brings the possibility of a flourishing of scientific literacy. At the very least, the capacity to inhabit a scientific frame of mind - to weigh facts dispassionately, to test hypotheses, to defer to data - will become crucial for all of us as the scientific knowledge proliferating at our fingertips shapes every aspect of our world. (218)

Likewise, Meredith Patterson, in the introductory quote for this section, includes literacy as a prerequisite for participation in her Biopunk Manifesto (2010). Literacy, for her, is not just the ability to understand but also to practice science, an ability that will "empower" people to become "active contributors" in "their very interactions with their own bodies and the complex world around them" (n.p.). It is interesting that she chose to start her biopunk manifesto with a reference to scientific literacy. It shows how also members of the DIY community position DIY biology as contributing a great deal to a more wide-spread scientific/biological literacy but also how intrinsically some people in the DIYbio community connect access to knowledge and social participation. Being 'literate' allows citizens to actively shape their bodies and surroundings.

Literacy is not just needed to make sense of the many different data about the body and private life collected today, but it is also needed to become engaged in molecular discourses of the body. On a personal level, literacy also goes hand in hand with considerations of informed choice - a topic widely debated in relation to healthcare: To be able to consent to medical procedures individuals would ideally require substantial knowledge of risks, side-effects, other treatment options as much as the procedure itself and its biological basis. Only if you understand what is done, you can give true informed consent to procedures or tests. Literacy, thus, should be a prerequisite for (medical) decision making, for example, in genetic testing. However, whether or not a consumer is truly enabled to understand the complexity, scope and short-comings remains relatively irrelevant for the testing process by DTC companies.

Literacy, moreover, is also a political question: As Patterson maintained, you need literacy for participation. Biological and technological processes can only be shaped once they are understood, which is especially important in societies that rely on medicine, biology, technology as much and as hopefully as Western societies do. Because these processes rooted in biology do not only have personal but also social consequences - new differentiations or valuations of life for example - access to resources and literacy to use them can shape social and political developments. For example, DIY's aim of participation and access questions the existence of a two-class society made up of those actors that possess knowledge and can thus determine (their own) fate and those that are disenfranchised from knowledge and thus cannot question decisions and practices. Increasing the literacy of the "public" is also seen as a means to redress the "democratic deficit," as Rose and Novas write, that exists when citizens cannot actively engage with and shape the future uses of scientific and technological innovations. Education strategies, thus, for them become a tool for the creation of "biological citizens" (Rose and Novas 445-46). 


\section{Performing Citizenship}

If we follow Rose, Novas and other scholars in their arguments, "citizenship" today is increasingly defined not just in somatic terms but also in a person's ability to participate in cultural discourses around the uses of bio-technologies. Levina, for example, in her discussion of network subjectivity in the control society - in the context of genetic testing - argues that (bio-)citizenship can also be enacted through participation in the network, by for example feeding it with new information (5). Williams uses the term "technocitizenship" to denote the "right and responsibility" of individuals to be informed about technological developments and take part in their governance and regulation (16). Citizenship, we can see here, is framed in biological and technological terms, which connects debates around the different uses of technologies to inherently political questions.

One of the most cited examples of these new types of "citizenship" is Nikolas Rose's and Carlos Novas' use of "biological citizenship." Happe et. al., in a 2018 edited collection on "biocitizenship," write that "biological citizenship" in its essence refers to how political initiatives, bottom-up and top-down ones, as well as technological developments have created "new ways in which bodies shape- and are shaped by-ideas of citizenship" (Happe et al. 1). Biocitizenship, for them, is today redefined as a "broad mode of political action" that is somehow "linked to health, bodies, and life" (Happe et al. 1-2). This definition certainly owes a lot to Rose and Novas' seminal work in the terrain. Rose and Novas further subdivide their understanding of biological citizenship into "informational bio-citizenship" - the necessity to possess specialized and medical knowledge about one's condition - "rights bio-citizenship" - involving forms of political activism and campaigning - and "digital bio-citizenship" - those forms of citizenship taking place primarily in the digital sphere (442). These different types of bio-citizenship, I would argue, are also "performed" or "enacted" (Ratto and Boler 3) in the DIY techniques under discussion in my case studies. Inherently physical, material, biological they are concerned with the governance of individual bodies as much as of science and society more generally. DIYers become "bio-citizens," in Rose and Novas' use, through self-education and the integration of specialized medical, biological and technological knowledge into their everyday lives; through political activism for openness, access, participation and a more just global distribution of scientific resources and technologies; and the creation of new communities, online and offline, around a common interest in biological materials and bodies. DIY biology, Morgan Meyer writes, creates a "distinct and political form of self" that is actively engaging with their own biological materiality and "making their bodies and ailments more knowable" ("Hacking Life?") - exactly the type of responsible, involved, self-caring and self-managing self that is required for scientific and biological citizenship.

Through DIY practices, therefore, citizenship is performed. Ratto and Boler look at DIY activities more generally as creating a type of "DIY citizenship:" DIY activities,

$7 \quad$ John Hartley, in his Uses of Television, also uses the expression "DIY citizenship" but for him this type of citizenship is tied to identity formation, in which the DIY citizen has more opportunities to amalgamate an identity and individuality from the available choices. Hartley argues for a flux of 
they argue, are political in that they "challenge existing systems [and hierarchies] of authority" and the status quo (5). "DIY citizenship" is used by them as an umbrella term for all sorts of practices that create new "possibilities of political participation" (7). Citizenship is no longer enacted only through what they call "standard political activities" such as voting, advocating or protesting, but has become more "diverse, participatory" and to some degree unexpected, in its locations as much as its practices (7). The maker movement, citizen science and DIY biology can certainly be considered as examples of such participatory and diverse performances of citizenship. Through their materiality they provide "new modes and possibilities for political and social engagement" (Ratto and Boler 16-18). This type of DIY citizenship, similar to Rose's insistence on biocitizenship's active nature, is focused on physical doing, on intervention, making, hacking and tinkering (Ratto and Boler 19). But DIY citizenship also continuously has to balance different interests and tensions: "between consumers and citizens, between experts and novices, between individuals and communities, and between politics as performed by governments and politics and DIY grassroots democracy" (Ratto and Boler 5). These tensions are also visible in the case studies in my discussion: the high involvement of lay persons challenges the authority of experts; new bottom-up transformations, as for example the "EpiPencil" or "Circadia" implant, stand in marked contrast to discourses of regulation and control; and DIY is both an act of consumption and a form of creative, political intervention.

However, "citizenship" of course is not immune to critique: According to Ratto and Boler, it is also a contested and loaded term that has often been related to forms of inclusion, exclusion and violence - a differentiation and differential treatment of those who are perceived as belonging, parts of the group of citizens (of a nation) versus those who are denied this right (8). Similarly, we can speculate whether or not DIY as a form of enactment of citizenship despite its promise of inclusion and demolition of boundaries might in reality create new types of differentiation and belonging - those 'enlightened,' enabled, active, literate, and responsible citizens versus those who are left out of these transformations (cf. 11.4). Happe et.al. also engage with this problematic nature of (bio)citizenship when they lament that scholarship on the topic often overlooks social inequalities (of race, class, gender, disability) and threatens to abandon collective engagement for individualized consumption (7-8). ${ }^{8}$ Biocitizenship, for them, is problematic because it is based on a "model biocitizen," who is rational, autonomous, healthy, able-bodied, and privileged, in terms of class and socioeconomic background (6). Tied in with the prevalent market rationality, this ability to care for the self, provide for one's own needs, makes the biocitizen a "quintessentially neoliberal subject" (Happe et al. 7). Instead of liberation from the decried status quo, DIY as (bio)citizenship might

identity and possibilities to change an identity. According to Ratto and Boler, on the other hand, his conception is very much rooted in Western ideas of self and privileges individualism and individual choice over structural factors (11-12).

8 Ruha Benjamin, for example, writes that scholarship on (bio) citizenship often looks at "a more elite strata of people who have the resources and influence to organize around their shared interests" and that "empowerment" is here primarily the ability to consume (17, cited by Happe et al. 7-8). 
thus further embed its practitioners in the ideologies they want to abolish, which potentially is visible already in the high prevalence of discourses of optimization and the latent consumerism. ${ }^{9}$ DIY citizenship thus might go hand in hand with problematic responsibilizations of the self instead of a focus on community and might facilitate a new type of coercion to make the self 'fit' for the demands of the market. These problematic connotations are not fixed but should rather inflect popular discussions of DIY biology and medicine.

\section{Promising Democracy}

Tied to the idea of citizenship, of course, is also the democratizing potential often ascribed to biohacking or DIY activities more generally. DIY can serve as a "democratizing agency," to borrow Atkinson's term (1), by leveling playing fields and allowing access for all. Like access, democracy of course is also a fundamentally American theme: Power and sovereignty, the American ideal goes, belongs with the people: "government of the people, by the people, for the people" as Abraham Lincoln famously declared in his Gettysburg Address. Morgan Meyer writes that do-it-yourself biology "is often praised for the potential it holds for democratizing science, ... for the empowerment of ordinary people" (Domesticating 2). This democratization, according to him, takes place spatially through domestication, community labs and, I would claim, an ensuing new definition of what a laboratory is or can be; technically, by redesigning technical equipment and creating more accessible alternatives; socially through its openness for everyone; and economically through decreasing costs for equipment (Meyer, "Hacking”). Paul Atkinson reasoned that DIY practices (in general) can work as democratizing agents by providing independence and self-reliance, freeing from professional interferences, allowing individuals to create "more personal meaning in their own environments or self-identity" and opening up boundaries thus granting access to all (5-6). Similar tendencies are observable in DIY biology and medicine: They exert their democratizing potential by creating cheaper, more economic solutions, freeing individuals from medical and professional supervision, fostering self-reliance and independence in research and application, and allowing people to engage more personally and meaningfully with their biological surroundings.

Christopher Kelty argues that biohacking as "new ways of science-making" and engagement with knowledge and tools changes not only science but also our conception of "the public." In DIY techniques, being part of the public is not passive but "aggressively active" (8). The Habermasian model of a liberal democracy needs "public spheres" in which citizens can discuss and debate matters of public interest (cf. Habermas referenced by Ratto and Boler 12-13). ${ }^{10}$ I would claim that community labs, online groups and

9 The discourse of optimization, I want to point out, is used also by biohackers themselves, as the case studies have demonstrated. In fact, some scholars argue that this discourse is quintessential for 'American' DIY biology (cf. 11.2).

10 A well-known critique of the Habermasian model comes from Nancy Fraser. She focuses on actual power relations, inclusions, exclusions, and systematic inequalities, the implicit gendering (through Habermas's strict division between public and private, excluding women from public participation) and the "problematic processes of consensus building," in Ratto and Bolers' words, 
discussion boards become such "public spheres" in which DIY citizens can deliberate on issues of common concern; but more than that, they become public spheres of activity - of doing, making, tinkering, physical production as much as theoretical deliberation. Though secluded, mystical, outlaw, DIY biology and medicine becomes an inherently public activity in which practitioners affirm their status as biocitizens. The idealized type of 'DIY democracy' espoused by DIY biology and medicine, I would argue, is characterized by equality, bottom-up processes of participation, diversity and a multiplicity of voices - a poststructuralist liberation from constraints (biological, social, cultural) and a challenge to established institutions. In this democracy, the self becomes more active in the configuration of all aspects of their life, including their biological foundation. This ideal of DIY citizenship and DIY democracy as an overarching concern of DIY biology and medicine points to the political nature of DIY itself, one that often goes back to questions of power, or rather, the subversion of existing structures of power in favor of new ones.

But critical voices need to heard, as well: Delfanti for example questions whether the opening of physical spaces, the increase in ways of participation, the challenge to mainstream science and the claims of access and democratization truly "consist of a real shift towards democratizing science" (Biohackers 120 ): Do they really change the power structures or only point out their problematic nature? Is, as Clare Jen writes, much of the "democratization project" of bio-making only "discursively produced" (127)? And if so, how much of that discursive production is done deliberately by DIYers and the media? How much democratization is realistic and how much only promissory? While this question is impossible to answer with certainty, it nonetheless points to the hype, the expectations and promises that these discourses of participation, access, and democracy carry with them.

\subsection{Can We Buy Safety? - Between Consumerism and Security Concerns}

"We reject the popular perception that science is only done in million-dollar university, government, or corporate labs; we assert that the right of freedom of inquiry, to do research and pursue understanding under one's own direction, is as fundamental a right as that of free speech or freedom of religion." (Patterson n.p.)

With its rise as a hobby in the 1950s and 1960s, DIY and making have also led to new economic possibilities for its practitioners, enthusiasts and service providers. Especially since hacking and making have entered the mainstream of society in the 1990 s and through the maker movement in the $21^{\text {st }}$ century, they have also become embedded in entrepreneurial activities. Selling tools, kits and resources, expertise and advise, access and knowledge have become part of the maker movement. As we can see in the case studies, efforts are underway to commercialize tools and materials and some techniques are already shaped by consumerism. Today, DIY biology, following other forms

required by the Habermasian model (12-13). I would claim that at least one aspect, the problematic division between public and private, is all but erased through the domestication of biology, but still some of the gendered concerns remain. 
of DIY, straddles an ambiguous position between consumerism and anti-consumerist activity. I would project that the incorporation into consumerism - the commercialization of DIY approaches and the exploitation of DIY principles for economic gain is only in its beginnings and can be seen as one of the potentially more controversial aspects of DIY biology and medicine. Similarly, if we look at public discussions around and media representations of DIY biology, commercialized or not, concerns about their safety and security, for individuals and society as a whole, are often central features of the narratives, whether or not such doubts are even warranted.

Commercialization and safety, it seems, are both particularly - but not exclusively - American concerns. Questions of (bio)security are to some degree rooted in American culture, especially American experiences in the $21^{\text {st }}$ century. I am here, of course, referencing 9/11, the anthrax attacks a week later and the ensuing war on (bio)terror. The rhetoric of constant, globalized threats to the safety and welfare of the nation and its people that was wielded in the aftermath of the terrorist attacks, have shaped contemporary American consciousness and have been applied to more and more domains with the increasing globalization and interconnection of the world - including biological materials. ${ }^{11}$ Concerns about DIY biology and medicine as a potential danger to the whole of the nation, Morgan Meyer writes, are more often voiced in the US ("Hacking" n.p.). Commercialization is a theme that is typical for 'American' biohacking as well: Especially in the United States the development of DIY biology and medicine during the past years has been mirrored by a rise in start-ups and other companies offering DIY services or DIY tools. ${ }^{12}$ References to such companies are frequent. Their founders are interviewed for media coverage or journalistic representations; they are highly present in the online DIY community and they are referenced in many of the guide books on DIY biology. Keulartz and van den Belt claim that in America there is a higher inclination to use DIY biology as a "launchpad" for start-ups than in Europe because the US model is closer aligned to entrepreneurship, market rationales and personal enhancement $(3-5,16)$. We could maintain that this is due to the entrepreneurial nature of American culture and society, in which entrepreneurial spirit is closely connected to values of self-sufficiency, autonomy and the "good life" (cf. also Ch. 4.1). In the US, DIY culture is part of a start-up culture and vice versa.

In this chapter I first want to discuss these topics separately - weighing their positive and negative sides as well as their potential - before creating a bridge between the spheres: On the first glance unrelated, commercialization and security can work together to spread and solidify the ideological basis of DIY biology. Even though DIY is often positioned in clear opposition to institutional science and Big Bio, this distinction does not hold up. Rather, this movement, this counterculture, has grown in symbiosis

11 Braun's argument, for example, merges biopolitics and geopolitics in the name of biosecurity, by looking at our (molecular) bodies as situated in a "chaotic and unpredictable molecular world" characterized by "exchange and circulation" and "haunted by the spectre of newly emerging or still unspecifiable risks" (14). Melinda Cooper considers the new preoccupation with bioterrorism/biosecurity in US foreign policy after 9/11, in a biological world full of cross-border movements. Morgan Meyer, for example, writes that DIY biology today experiences a slow rise in market logics and that companies have begun to form around the needs and demands of the community (Domesticating 14). 
with the 'big players' - with potentially transformative effects for systems of knowledge production in the whole industry. Similarly, as agents of familiarization commercial alternatives integrate unruly and unregulated practices in well-established systems and orders that could be exactly what is needed for the movement to gain momentum and become more 'mainstream,' thus moving closer to the ideology of access for all.

\section{The Ups and Downs of Commercialization}

Commercialization does provide a clear advantage for DIY biology and medicine, which should not be understated or underestimated: It makes DIY approaches more visible in the public, makes them more accessible to more people and lowers the hurdles of participation. Commercialization can grant new access to knowledge and tools, for example through easy-to-use DIY kits. Instead of having to set up a laboratory at home or finding one of the few in one's community individuals can purchase such kits or technologies and have all the tools they need to conduct a specific experiment. Through commercialization people can much more easily engage with biology or their own bodies. Consumerism can make DIY even more available, accessible and productive, which would turn it into a key building block for DIY biology's promise of democratization. Jonathan Bean and Daniela Rosner distinguish between making as a (social) movement and making as a brand. Movements, for them, are social groups focused on a desired (social) change and "creative empowerment" from the bottom up (26-27). Branding's aim on the other hand is commodification and the attachment of meaning through a top-down approach (26). In the case of making branding, for example, tries to sell making as a mindset, provides possibilities for economic expansion and creates new potential customers for tools, magazines, and other resources (27). Bean and Rosner argue that making has become a good brand, one that sells, one that provides meaning for customers/makers, and one with transformative potential in that it could lead to an ideological shift in which "consummakers" can change the world through production (27). DIY biology and medicine, in this line of thinking, can therefore be both (social) movement and (commercial) brand.

However, this type of branding through commercialization - though successful could also transform making and DIY from an egalitarian and open movement into a product dominated by the market. In neoliberal societies, consumption is a "technique of the self" (Foucault), a means to perform and signal a certain identity. DIY has become such a "technique of the self" as well. To remain competitive, work on the self is mandatory and this work today is often done in the form of DIY interventions and practices, from fitness tracking to genetic testing or biological experimentation with one's own body. Through consumption, especially of goods that promise to alter, manipulate or monitor the body, we can ascertain choice in a very direct way - by individually choosing services and goods that we, as consumers, deem necessary and beneficial to our individualized life projects and identity performances. According to Rose, markets and consumerism are also perceived as means of personalization because they (seemingly) increase the power of individuals and allow them to choose desired services, goods, or providers ("Personalized Medicine" 341). But as Rose also contends, this choice might only be superficial: The increasing responsibilization of individuals for their own well- 
being and physical health creates not just a market of promises, but also a "market in anxiety" (Chrysanthou 476). Consumption becomes not a choice but a requirement if one wants to partake in the former and avoid the latter. These markets, moreover, are brimming with professionals seeking to make a profit. As some of the case studies have shown, commercialization also leads to the creation of new "experts of the soma" and "economies of vitality" (Rose, Politics of Life 6-7, cf. Case Study I). DIY biology gives rise to a new class of professionals dedicated to performing and furthering it. Consultants, teachers, coaches might fill the void left by the shift away from older figures of expertise (such as doctors). Through this 'side effect' of commercialization, new economies arise around the individualized concern with the human body, with new means of generating (bio)value.

Conversely, commercialization might also do away with many of the transformative principles of DIY, create new (financial) barriers to access, restrict its openness and hacker ethos. In typical commercialized settings such as the wearable technology industry proprietary software and platforms restrict access to your own information as well as the possible uses and 'tinkerability' of the tools. Openness is taken over by profit as a goal. Many of those prototypical commercialized applications of DIY medicine and biology, such as genetic and molecular testing, can also be considered as a form of free labor, the "donation" of work, free time, creativity, data and bodies to the enterprises of businesses, research bodies and start-ups in the name of openness, sharing and distributed innovation (Delgado 72). Such practices of sharing, of course, open questions of data security and privacy, how data are (mis)used, stored and sold. Could they be used by states and other actors for biopolitical strategies and coercive practices? In commercial settings self-gathered data, for example in self-tracking practices or genetic testing, are in the hands of companies and could be misused without the user's (informed and explicit) consent. Especially in contributory and co-created research models, consumer-oriented systems for data collection pose risks of surveillance, monetization by providers, and lack of informed consent (Wiggins and Wilbanks). Tensions emerge between data ownership, data sharing and data privacy - tensions in which "data philantrophy," donating data for the greater good, is positioned as the right, the beneficial and solidary thing to do (cf. Ajana). The DIY research "revolution" proclaimed by 23andMe is a good exemplification of such discourses: Sharing your data will benefit society at large by helping to find treatments or cures for diseases. In such cases, these companies use the 'label' of DIY without implementing its ideology - similar to the trend towards 'greenwashing in other consumer segments - which might create negative effects: Beguiled by the promise of empowerment and participation, consumers might be trapped in new unequal power relations and interdependencies.

\section{Co-optation's Transformative Potential}

While DIY biology and medicine are often perceived and portrayed as a challenge to traditional approaches in the practice of science and medicine - a form of "counterculture" to biocapitalism with transformative potential (Delfanti, Biohackers 112-13, 129) Delfanti finds that DIY biology cannot clearly be separated from those traditional institutions of science, the medical market or state bodies. Instead, he claims that it is in 
relation to all of them, either participating in, relying on or foreshadowing future possibilities ("Do-It-Yourself"). This close connection both to the (biomedical) market and institutionalized forms of science is observed by many scholars analyzing the DIY biology movement. ${ }^{13}$ According to Delfanti biohacking is not working against entrepreneurship and profit, but rather combines it with a new ethos of sharing, distribution, and participation - without however, closing the door to financial gain. Against this backdrop, Keulartz and van den Belt question whether DIY can even become a true alternative to Big Bio or whether it will in the end become part of the vortex of biocapitalism and commodification (16). My case studies have demonstrated this deep entanglement with for-profit efforts as well. DTC genetic testing, once more, is one of the most obvious examples of how money is made from the participation in and donation of personal data in the name of consumption. "So is it disingenuous to build a subculture atop such a massive institutional foundation and call it DIY," Wohlsen asks (120)?

Delfanti argues that the close connection between biohacking and existing institutions "signals a process of ongoing co-option" in which DIY biology is increasingly incorporated by the bio-industry ("Do-It-Yourself" n.p.). Co-option, he writes is a phenomenon that typically affects social movements that try to effect social change through technological innovations. These technological innovations, often developed with some type of help from industry, are then incorporated into and adapted to industry needs. Because DIY biology's position in relation to scientific institutions, markets and the state - or rather its enmeshment with these actors - seems to stabilize, Delfanti maintains that DIY biology might undergo exactly such a process of co-optation, potentially resulting in the loss of its "innovative tension" through the absorption of its technologies and practices ("Do-It-Yourself" n.p.). For DIY, this might mean a loss of its transformative potential.

If Delfanti's prediction proves true, co-optation could lead to larger attempts to integrate the DIY approaches into existing structures and systems of power. But can DIY approaches be integrated (productively) into the current economic system without losing their ideology? According to Boltanski and Chiapello (2005) capitalism "feeds" on critical cultures: The incorporation of critical cultures helps "restructure and renew the ideological and organizational logics" of capitalism, as the example of 1960 counterculture has shown (cited in Soderberg and Delfanti 3). Soderberg and Delfanti argue that

13 Ana Delgado claims that DIY bio is characterized by an ambiguous relationship to institutionalized forms of biology, because it depends on and questions them at the same time (67). Keulartz and van den Belt observe this ambiguity as well: While DIY biology's ethics, values und practices seem to be in contrast to Big Bio's focus on profit, DIY biology is also dependent on Big Bio as a source of second-hand equipment and rapid technological development and DIY bio community projects are increasingly turned into businesses (3-5). Kelty argues that DIY bio is "enabled by and thrives upon the infrastructure of mainstream biology" (1). For example, there are many personal and professional connections between DIY bio labs and "professional scientists" as many participants are often researchers or students. Meyer also points to the many entanglements of DIY biology with "professional science" and Big Pharma: Despite its critique of the very logics of this industry, its closed off nature and lack of access - an ethos of "do-it-without" as he terms it - DIY biology also relies on it for the purchase of used equipment, tools and products or by accepting donations from companies ("Hacking"). 
a similar process is happening with hacker culture and potentially maker culture (3). In Biohackers, Delfanti calls this a process of "recuperation" - the instrumentalization of a critical culture (14). Cultural transformations such as DIY and making, that pave the way for a "participatory, cooperative turn of informational capitalism," can through co-optation and incorporation influence the progress of today's capitalism (Delfanti, Biohackers 50). The end point of such co-optation processes is a situation in which it becomes almost impossible to separate the more radical practices of DIY from those commercialized ones. The boundaries between DIY and DTC have become blurry and both extract commercial value from information, biology and bodies.

Commercialization can be limiting and liberating and also co-optation can have a transformative impact by changing culture on a larger scale. Open biology, following Delfanti, could thus become a "tool of resistance" while at the same time participating in neoliberalism and capitalist value creation (Biohackers 14). As some of my case studies have shown, DIY and consumerism can actually be working together, following similar goals. They do not have to be dialectically opposed but can form a fruitful combination. DTC can be a precursor of DIY practices and vice versa, as the integration of DTC testing into DIY, citizen science clinical trials or personalized regimes of self-transformation has shown (cf. Case Studies I and V). The co-optation of DIY principles into neoliberal capitalism could, as the discussion above has shown, actually change the current system into a more participatory, social, egalitarian one, suited to address some of the most pressing concerns of today. I propose to see DIY approaches and DTC approaches as nodes in the same network: Techniques that in their own way further an agenda of participation and empowerment, both with their own drawbacks and downsides. Working together - in their network - they can potentially elicit much more large-scale developments than individually. This combination can also, as we will see, help to alleviate some of the anxieties around DIY biology and medicine in public discussions.

\section{Security and Fear}

The contemporary conception of biomolecular life not as fixed but as fluid turns biology from something calculable into a "a dynamic world characterized by ever novel combinations, where entities jump between bodies and cross between species, and where life itself' continuously confronts us with the new and the unknown" (Braun 17). These biological "unknown unknowns" (18) that could come from everywhere, Braun contends, incite fears and a political rhetoric of threats to life and liberty. ${ }^{14}$ The focus on "biosecurity," thus, can also be considered as a (political) response to the unpredictability of

14 The "unknown unknowns" that Braun takes up here (presumably) go back to a statement made by United States Secretary of Defense Donald Rumsfeld in a Department of Defense news briefing in February 2002 in relation to the war in Iraq and the lack of evidence for the presumption that the government of Iraq supplied terrorist groups with weapons of mass destruction. Rumsfeld argued: "Reports that say that something hasn't happened are always interesting to me, because as we know, there are known knowns; there are things we know we know. We also know there are known unknowns; that is to say we know there are some things we do not know. But there are also unknown unknowns - the ones we don't know we don't know. And if one looks throughout the history of our country and other free countries, it is the latter category that tend to be the 
molecular life - also through globalization (Braun 15). Making biology more open and accessible, some critics claim, "also makes it less predictable, raising the specter of unknown dangers" (Bennett et al. 1109): Fear of invisible dangers, concerns about the selfdirected nature, worry about the welfare of the nation more generally are part of the public discussions around DIY biology. As Bennett et al. write, "[i]n certain respects, DIYbio is a 'black swan' waiting to happen: it portends events whose probability might seem low, but whose negative impact is likely to be quite high" (1110). These security concerns are also shaped by the common understanding of 'hacking' as a rather dark, illicit activity. Prominently, these discourses come together in the figure of the (bio)terrorist to emphasize the unpredictable nature of this danger (Meyer, "Hacking"). In the public imagination, DIY biologists, especially those working with those wonderous, minute substances of life, are also potential bioterrorists who work in a black box of elite technology and reclusive hacker ethos.

Often, cultural representations of DIY biology tend to escalate what is done in DIY labs and homes of biohackers, so that we find a stark contrast between the public imagination - genetic engineering, CRISPRing organisms - and the real-life practices of many biohackers. DIY bio activities are represented as both potentially revolutionary and "extremely dangerous" (Aguiton and Tocchetti 834). Media discourses show them as a promise and a threat. For example, the specter of biological engineering is already visible in the treatment of DIY biology in the American show Designated Survivor in 2019, but much more prominently in Biohackers (2020). The trope of 'god-like' powers through synthetic biology is included already in the first episode of the streaming series: "Synthetic biology turns us from creatures to creators," Prof. Lorenz preaches to a full auditorium. ${ }^{15}$ The biohacking that is performed in this show, then, also includes the unregulated genetic engineering of mosquitos as well as self-made genetic treatments for diseases. While these cultural representations are often science fiction, they do speak to a desire that is also prevalent in parts of the DIY biology community. With easier access to sophisticated technology, we are also moving closer to recombinant DNA and genetic engineering - those controversial issues that form the basis for many of the security concerns around DIY biology. Indeed, the availability of more and cheaper tools needed for complex genetic experiments is reflected in what is sold to new biohackers. Josiah Zayner's company The ODIN, for example, sells DIY CRISPR kits that allow people to genetically-modify organisms in their home. Zayner received a lot of media attention for his highly publicized and controversial hacks, such as injecting himself with CRISPR

difficult ones." (Secretary of Defense Donald H. Rumsfeld, DoD News Briefing, Feb 12, 2002). This statement has gained cultural notoriety since then.

15 Her life-long goal, we learn, was to eradicate genetic disease, amongst others with a gene therapy program called "Homo Deus" that was used on embryos - deadly to all recipients but one. This therapy, it turns out, can also give people a stronger immune system. The intent was a cure, the result is an enhancement. Synthetic biology and genetic engineering, as Biohackers fantasizes could give rise to new types of human enhancement that builds inequalities literally into the biological make-up of humans - genetic optimization will become the norm, as Prof. Lorenz maintains. We might also want to note that the name given to this program "Homo Deus" resonates deeply not just with Harari's eponymous non-fiction book but also the desire he describes in it: to create more perfect beings, God-like humans. 
on stage in an attempt to do genetic engineering on humans. The kits sold by The ODIN include experiments that would modify non-pathogenic E. Coli bacteria to survive on a medium they would usually not survive on or to engineer yeast to become fluorescent ("The ODIN"). ${ }^{16}$ Such possibilities, while relatively basic today, feed into the prevalent wariness or even fear of dangerous biological particles and the risks associated with unregulated, DIY experimentation.

In these discourses of risks DIY biology is frequently seen as a danger to public health and environmental safety as well as national and personal security (Meyer, Domesticating 3; Wolinsky 683; Gruber). These dangers are reflected in the three kinds of risks that are commonly identified when it comes to synthetic and DIY biology: first, the accidental release of potentially harmful organisms due to lower safety precautions in typical DIY settings; second, a deliberate release with good intentions but unintended or unforeseen side effects on the environment; and third, the deliberate and malicious misuse, for example through the fabrication of new pathogens and their release (Stevens 351-52). ${ }^{17}$ Moreover, critics are also concerned about the consequences for individual bodies, for example when untested technology such as the "Circadia" implant is integrated into the body, hacks like the DIY "EpiPencil" used as substitutes for regulated emergency medications, or The ODIN's DIY CRISPR kits used for genetic selfengineering. ${ }^{18}$ Interestingly, similar concerns about personal safety were also present during the rise of DIY home improvements in the 1950 s and 60s, when the broader availability of power tools also gave rise to concerns about the potential dangers to personal safety that resulted from their use (Gelber 278-82). DIY biologists counter safety concerns with hints at the harmless nature of their experiments and the (as of now) functioning forms of self-regulation in the community. In the US, this often results in a discussion between state interference and freedom of choice, freedom of speech and personal autonomy, because, as Stevens writes, in the age of recombinant DNA and genetic engineering biosecurity would require regulation of information and not just

16 On a side note, the import of DIY genetic engineering kits into Cermany - such as those from The Odin - is prohibited and their use outside of laboratories can be fined up to 50,000€. German authorities have warned of the CRISPR kits because of disease-transmitting bacteria that were found in them and the FDA has issued a warning not to use self-administered CRISPR kits as well. Almost all researchers that concern themselves with safety and security issues stress that they can happen accidentally as well as intentionally (Wolinsky 683; Bennett et al. 1109-10; Stevens 351-52). However, we also need to take into account that as of today, it is not really feasible to engineer new pathogens from scratch to use them as biological weapons, especially because already existing biological materials, such as anthrax or castor beans, could be used for similar purposes with much less effort. But, as Baumgaertner suggests, with increasing numbers of hackers and sophistication of technologies, the potential for abuse might grow (n.p.). In fact, scientists at research universities have already managed to engineer an extinct pox virus from mail-ordered DNA fragments. The series Designated Survivor takes up this cultural imaginary of newly engineered threats.

18 In a report for the New York Times from July 2019, Emily Baumgaertner implies that Josiah Zayner, founder of The ODIN, is aware, even afraid, of the potential for people to get hurt when people uncontrollably try to "one-up each other" with their hacks. In another report for VOX in June 2019, Sigal Samuel ("How Biohackers") quotes Ellen Jorgensen's - co-founder of the biohacker space Censpace in New York - warning about the potential dangers of DIY CRISPR on human genomes. Biohackers, it seems, are aware of and addressing the potential dangers ("How biohackers"). 
materials (Stevens 353) - thus potentially impinging on all of the former. Patterson's argument that freedom of research is as fundamental a right as freedom of speech is a case in point. DIY biology and medicine - or rather the increase in scientific literacy that results when "scientific knowledge seeps into the public domain we all inhabit" - can turn these debates about "scientific risks and benefits" into a collective, social responsibility, as Wohlsen concludes in his afterword to Biopunk (217-18).

\section{Familiarizing Biological "Unknown Unknowns"}

What the discussion of commercialization and of safety goes back to, I would argue, are questions of location - outside and inside - and approaches - top-down and bottomup. Consumption often is a top-down approach in which companies decide what becomes available (even though consumers do have a say in types of demand), while DIY solutions are characterized by their bottom-up nature. Moreover, commercial solutions could be characterized as inside (the system), while DIY solutions are typically perceived to be outside of established systems (even though, as we have seen, that is not necessarily true). In the public perception, top-down and inside are associated with safety, regulation, security; while bottom-up and outside are associated with potential danger, rebellion, anarchy. As a consequence, commercial approaches are evaluated as safer and less controversial while DIY approaches are deemed more dangerous and unregulated. Such safety concerns might be countered by co-optation and commercialization: commercial devices are not just subject to stronger regulations but also work as agents of familiarization. Unruly DIY practices are integrated into a well-established system; they are no longer out there and dangerous but part of a trusted order.

To some degree, the DIY community in the US has put those distinctions to work for them, even if subconsciously. They have countered fears about the safety of their practices by working with established players and regulatory bodies. The DIYbio movement, for example, has established a strong (working) relationship with the FBI and other government bodies and at the same time positioned itself as an educational community with potential benefits for the whole of society (Delfanti, Biohackers 116). Aguiton and Tocchetti focused on the relation between DIYbio and the FBI as an example of the entanglement of promissory biotech and biosecurity risks: ${ }^{19}$ During the collaborations between FBI and DIYbio, their argument goes, a "trading zone" was opened up that relies on the "entanglement between promise and risks and enables a relationship of opposing entities to collaborate." The collaboration with the FBI furthers DIYbio's technological promises by "governing" their activities - quieting down fears about biothreats also in the media and thus enabling the "true" message of DIYbio to be heard and the network to grow - and DIYbio helps the FBI by policing themselves and their

19 According to Aguiton and Tocchetti, this collaboration reached from informal visits of FBI agents to community meetings, presentations by the FBI during conferences and community events, to jointly organized gatherings of global biomarkers. Interestingly, the collaboration between these contradictory actors has also made it into popular culture: The third season of Designated Survivor, referenced in the introduction, thematizes the problem of regulation and brings together a $\mathrm{CIA}$ agent and a biohacker to counter a possible bio-thread to the United States. 
members. They have established a system where suspicious behavior can be reported to the FBI, thus allowing the FBI to more efficiently survey the activities of the community for potential dangers (Aguiton and Tocchetti 829). In this intermediary zone outside and inside mingle so that both parties gain from the collaboration: DIY biology is conferred with the authority of an 'inside' institution that helps mitigate public perceptions of danger, while the FBI gains the trust of an outside and unruly movement. This, however, could also be a form of co-optation because the innovative practices and approaches of DIY biology are integrated into networks of policing and governance that run counter to the creative, unconventional hacker ethos. In the US, on the other hand, this type of co-optation might be necessary for the movement to gain credibility and grow in spite of the present anxiety around bioterrorism.

\section{3 "Obliged to be Free" - Empowerment, Choice and Precarity}

"Biopunks experiment. We have questions, and we don't see the point in waiting around for someone else to answer them." (Patterson n.p.)

Patience, it seems, is not the strong suit of many biohackers. Instead of passively waiting for technologies that address the questions they have or problems they experience, they want to take an active part in this development. Nowhere is this more apparent than in the NightScout and OpenAPS movements with their moniker "\#wearenotwaiting." What this impatience points to as well is a pervasive discontent with their current situation, personally as well as structurally. For a long time, (diabetes) patients in this example were expected to wait for the doctor to make their diagnosis, prescribe a therapy and monitor the progression of disease. Armed with the internet as information tool, DTC tests as monitoring devices and DIY instructions for supplementation, lifestyle changes and medical devices, these patients are now more than ever entering an age of empowerment with more choices, more possibilities to actively shape their health (care) - at least so it might seem. In this part, I am turning to the personal consequences for the self that DIY biology and medicine might have. This includes questions of power and empowerment, authority and agency, choice and responsibility, a deeper knowledge of the self as well as a new sense of vulnerability and uncertainty. Are we "obliged to be free" (Rose, Governing 217)?

I will argue that the new power granted to individuals through DIY dialectically fosters feelings of vulnerability, precarity and uncertainty. Through the lens of empowerment, DIY becomes a means for individuals to self-determinedly and self-responsibly influence different aspects of their lives. As such, it can become a new means of wielding power in the $21^{\text {st }}$ century. This goes hand in hand with changing relationships between experts and laypeople - as the latter claim more and more authority over the fields of the former as well as their own lives and bodies. A potential downside of this process is the increasing responsibilization of individuals (which always risks neglecting social and structural factors in favor of personal ones) as well as the rise in horizontal, peer-controlled power structures. The new authority of laypersons, on the other hand, entails new types of knowledge of the self that give rise to ideas of modification and 
optimization. Both the knowledge and the quest for modification can lead to new forms of corporeal precarity that undermine the promise of control and hope perpetuated by the discourses of empowerment. Rather, it leaves the body vulnerable to inside and outside forces. "Precarity" is used by Judith Butler to denote such states of increasing dependency and vulnerability to outside forces, a loss of social rights and material securities (Delgado and Callen 180-90; J. Butler, Precarious Life). ${ }^{20}$ Such a state of precarity is induced by DIY and its discourses of empowerment, agency, responsibility, and selfknowledge in multiple ways, as my discussion will show.

\section{Informal Experts}

DIY necessarily raises broader questions of (individual) power as the do-it-yourself always implies some sort of empowerment to take on tasks yourself that previously were carried out by professionals. "Taking ownership of their own biology" and the process of demystification are said to be deeply empowering (Wolinsky 683). Through DIY techniques, as my case studies have shown, individuals gain agency over their bodies and choices, take control over different aspects of their lives in which they previously often had felt powerless. This empowerment of marginalized actors - patients, lay persons - also takes place through technology and knowledge and is thus inherently related to questions of access and participation. Having knowledge becomes a prerequisite for gaining power and control. ${ }^{21}$ Through its central premises, DIY biology and medicine, thus, becomes a form of playing out and reshuffling power relations, a redistribution that throws into disarray existing structures - or at least is a symptom of the broader changes in the systems of power during the last decades, a new rendition of the discourse of individual empowerment so prevalent in contemporary culture.

Through the increase in individual power and agency, relationships between professionals and lay persons - be it scientists and society, medical professionals and patients - are transformed from paternalistic to egalitarian, an inherent goal of DIY aspirations. Stevens muses that through movements like Do-it-yourself Bio and citizen science clear distinctions between experts and the public might be resolved (11). As "boundary movements" they "obscure" the boundary between experts and laypersons and instead create a space for fluid movements between these worlds of knowledge: Activists in such (social) movements become "informal" experts through the gathering of information or direct engagement with scientists and medical professionals (Brown et al. 597). For example, Lupton writes that the "quantified patient" is an expert on their own body,

20 Precarity, according to Delgado and Callen, is also part of the material conditions of the "DIY scene": They write, that "[w]hat is rarely emphasised is precarity as the material context of existence of the DIY scene," amongst which they include the precarity in traditional research institutions - the lack of funding and jobs in academia, the lack of research opportunities for young scholars, and the immense pressure to publish. In this setting low-cost, creative hacking of biology has become an alternative, in which biology and the lab are "owned" and "appropriated" (180-90). But I here want to focus on personal consequences, not broader social contexts, even though I do think that these need to be addressed sooner rather than later.

21 I understand power in a Foucauldian sense as pervasive and differentially distributed power in complex networks of actors, discourses and knowledge practices. 
more knowledgeable about it than their doctors, which alters and questions the very foundations of this sacrosanct relationship (Quantified Self 78). Similarly, tinkering, the primary mode of inquiry in DIY biology and medicine, is by Christina Dunbar-Hester understood as a form of "cultural production" that aims at producing more egalitarian social relations.

The consequence of these transformations in traditional relationships ranges from less dependence on expertism on the side of the patient or public towards broader structural changes towards egalitarian, cooperative social relations - a dissolvement of (knowledge-)elites in favor of a more equal society. These shifts in (power) relations are not only found in traditionally overtly hierarchical power structures such as the doctor-patient relationship. Instead, we also find it in the scientific community, and indeed society, more generally. In science, Erickson demonstrates, we increasingly find a "twoway street" between esoteric and exoteric communities that now work together to produce new knowledge (2-3). The distinguishing feature of the scientific community, the "scientific method," as it is increasingly recognized, of course can also be employed by lay persons in the pursuit of new insights. DIY biology and the citizen science movement show that it is no longer the exclusive domain of formally trained scientists. The result are new concepts such as the "prosumer" - a hybridization that combines knowledge production and consumption as a new role in the production, dissemination and transfer of knowledge (Brüninghaus and Heyen 63, Toffler 284). ${ }^{22}$ Through the erosion of these boundaries DIY challenges the very foundation of the differentiation between the scientific communities and society. What does that do to our understanding of science and the belief in scientific solutions? Will it lead to cultural skepticism about the validity of scientific findings, about the quality criteria for research? Will it cement scientism as the dominant epistemology of our time? Or will transparency further distrust and skepticism? These questions remain to be answered.

Despite the many positive outcomes of empowerment and transformations in asymmetric power relationships for the individual and society more generally, also some of its negative sides should not be discounted and in fact might play a crucial role while new principles and guidelines for medico-scientific encounters between different actors are discussed. For one, the loss of power on the one and the increase in power on the other side often does not happen without resistance from those previously in power. Also in the 'original' home improvement movement conflicts arose between professionals and DIYers, where professionals urged the DIY community to recognize their limits often with a view to their personal safety (Gelber 275). Similar push-back might arise in parts of the scientific and medical communities. Other concerns include

22 Anne Brüninghaus and Nils Heyen argue that people no longer consume scientific or medical knowledge in order to simply inform themselves or form an opinion, but also in order to take part in the production of knowledge themselves: This leads to new forms and roles of production and consumption of knowledge, in which people become "prosumers" that interact differently with received notions of expertise and knowledge transfer. This shift could potentially change how knowledge is transferred and communicated (Brüninghaus and Heyen 63). This new role somewhere between expert and lay person challenges the clear division between the two and heightens the importance of non-certified expertise. Knowledge production and consumption are no longer a one-way street but active participation is required. 
the intentional mis-use of technologies and 'unprofessional' uses as well as the potential employment of technologies for negative goals made possible through a lack of professional oversight, leading to slippery slopes in the direction of coercion to comply or new eugenics. An anti-elitist movement risks being exploited also by disingenuous voices: Especially in the case of self-proclaimed experts and hacker 'gurus,' the borders between fact- or evidence-based medicine and new forms of quackery become blurred. Body hacks might no longer be backed by science or unproven methods marketed to uninformed audiences for economic gain. The booming wellness industry is a good illustration for such practices, where decisions are often made based on promissory marketing and anecdotal evidence. ${ }^{23}$

Fittingly, as Deborah Lupton suggests in her consideration of Medicine as Culture, some critics argue that the regulation and "professionalization" of medicine has the positive effect of ensuring a minimum standard of expertise in persons allowed to practice as medical doctors thus protecting patients (109). The medical encounter, proponents of 'older' structures claim, depends on the differential distribution of power and knowledge between patient and doctor. Those roles, constituted by practice and discourses of medicine, rely on the doctor being the expert. In their arguments, leveling the power relations destroys the purpose of medicine: Why would people consult medical professionals if they have equal knowledge? If patients continuously question the role and position of the doctor, "how can the doctor-patient relationship exist"? (Lupton, Medicine 116-17) Posed in 1994, these questions remain relevant until today, maybe even more so through the easy availability of medical information online. DIY and its focus on collaboration might provide a solution in which both doctors and patients are recognized as experts in their own right that equally participate in and contribute their knowledge to the medical encounter. Whether or not such a solution is feasible on a broader scale, however, is debatable and also hinges on the promised literacy and acceptance in the medical and scientific community.

\section{Vulnerable (by) Choice}

What comes with the discourses of empowerment and agency is also a perpetuation of the focus on individual choice and responsibility, criticized already by Rose. Following him, we are today no longer looking at the specter of eugenics with its state directed, top-down interventions into the health of the individual and nation but at a biopolitics based on individual choices, at least on surface value. If the choice lies with the self, the responsibility for the positive or negative outcomes of the choice lies with the self as well. The capacity to "care" for the self is in neoliberalism anchored in the self as a

23 The Netflix documentary (Un)well tries to shine a light on the claims and promises made by the wellness industry. (Un)well sets out to closer analyze the promises made by the multi-billion wellness industry and asks whether or not its practices actually make us well or unwell. Examples in this 6-episode mini-series include: essential oils, tantra, breast milk for grown-ups, intermittent and water fasting, the hallucinogenic plant-extract ayahuasca, and bee sting therapy (Druckermann et al.). All these examples could be considered as CAM, they are self-chosen and often selfadministered - a typical example of DIY values. The series has been criticized for reproducing a false balance by drowning out critical voices with enthusiastic, more benign practitioners. 
rational actor (cf. Jen 136). On the first glance, the DIY approach necessarily entails such agentic individuals. But some of these techniques are embedded in larger webs of power and control that extend beyond the individual towards their peers and governments.

What comes to mind is Foucault's concept of "docile bodies." Foucault's studies have shown that power in the medical encounter is more pervasive and subtle than previously thought because it is not just enforced by authoritative figures but also through individual self-surveillance and self-policing. Governmental management was coupled with self-regulation. The "disciplined" or "docile bodies" that arise out of these contemporary, less openly coercive strategies of biopolitics are rendered a target for mechanisms of power that reach into and shape their bodies, desires, identities and concepts of self (cf. Pitts 36-38). Docile bodies are not just disciplined but also self-disciplining (cf. Steinberg 14). Through responsibilization, we increasingly encounter forms of horizontal control between peers in which non-conformity to what ought to be (based on calculations) is punished (Selke). The heightened focus on the self and the body, its comparison to perceived norms and others, makes individuals more aware of their own bodies and invites a deficit-based thinking. This type of thinking is already deeply embedded in self-help discourses with their structure of lack and lack-rectified. A focus on our shortcomings and optimization potentials, however, leaves the body in a perpetual state of uncertainty, never enough and always on the verge of becoming better. Health is "recast as a perpetually insecure state" (Schüll 318). ${ }^{24}$ Deficit-based thinking invites a constant self-surveillance. As "potential patients" we must be constantly alert to bodily changes, whether or not we are sick or healthy (Pitts-Taylor, "Medicine" 164). This surveillance, however, also includes those around us. In a form of "sousveillance" people watch each other, make judgements and compare (Lupton, Quantified Self 58-62). In a "net of surveillance" people (users of tracking devices in particular) turn the medical, panoptic gaze upon themselves and their peers through practices of monitoring and sharing (Sharon 98) - leaving individuals in a sphere of constant monitoring and feedback, vulnerable to their own and the other's gaze.

The underlying question, thus, is one that already Braun posed in relation to Rose's ethopolitics: "What does it mean to 'exercise choice' in the self-management of the body?" (12) What defines a choice, how do discourses impede on this choice? Is it really an individual choice when we take into account ideas of horizontal control? How free can choices be in such a context? ${ }^{25}$ Rose writes that "responsibility is a double edged sword" that "seems to give people more power as individuals" while also obliging them to take on this role as an active, involved, responsible subject ("Personalized Medicine" 349). Choices might be severely constrained by the pressure of peers, social obligations

24 Dumit similarly writes that we have experienced a shift in the conception of illness: Bodies, today, are "inherently ill, whether genetically or through lifestyles or traumas," they are inherently insecure (7). Health and illness, for him, are "epistemic," no longer "states of being" but "states of knowledge" (13-14). As an epistemic practice itself, DIY has and increasingly will become a new avenue through which we can arrive at that state of knowledge.

25 Taking this idea further, the increasing reliance on technology also impinges on the idea of free choice. To make choices about the body individuals increasingly rely on technologies: Schüll even argues that this dependence on technology in wearable tracking turns individuals from an active choosing self into a passive one that needs devices for help (330). 
and cultural discourses. For example, in the context of personal genetics, you can, as Henk ten Have writes, no longer claim to be "victim" if you have "deliberately decided" to forgo predictive measures, such as genetic diagnosis - it was your choice "not to know" and "not to eliminate potential disadvantages" (301). The consequences have to be carried by you and no one else. The individual is obliged to act responsibly, choose carefully and it is this obligation that makes them not free but actually very much limited. "The self is not merely enabled to choose," Rose argues, "but obliged to construe a life in terms of its choices, its powers, and its values. Individuals are expected to construe the course of their life as the outcome of such choices, and to account for their lives in terms of the reasons for those choices" (Governing 231). They are "urged to shape their lives through choice" but often lack the knowledge to "navigate" those choices (Schüll 324-25), leaving them with a "double insecurity" of "always being at risk" while "never knowing enough about what one could and should be doing" (Dumit 1-2). If the "obligation to choose" becomes an obligation to act according to what one knows, the "freedom" to choose to know more and potentially optimize what is not going according to norms is not really a freedom at all but an obligation.

The idea of "choice" is most problematic, as in many biopolitical questions, if we take into considerations those who "lack the resources that might enable them to choose:" For them, "the biological self is a precarious entity - bare life, exposed to death - rather than an object of personal reconstruction," argues Braun (12). The focus on individual choice risks negating social and structural factors that impinge on the body and life choices. The promise of control through technology and/or DIY techniques is often used to amend such feelings and realities of precarity. The belief (almost religiously) in scientific solutions for bodily ailments and limitations is used to come to terms with the inherent vulnerability of the 'human condition.' For example, as Lupton asserts, self-tracking - by turning "fleshy sensations" into data - "becomes a way of mastering the uncertainties, inaccuracies and vagaries of human embodiment" (Quantified Self 54). Similarly, also other DIY techniques, such as implantable monitors, hacked medical devices or molecular testing, can be used to counter uncertainty with technology.

\section{A Precare Existence}

The idea of more control, as Lupton finds, is also based on the availability of more data about the self, a higher degree of self-knowledge that, it is perceived, allows individuals to assert control over their bodies and lives in an unprecedented manner (Quantified Self 67-77). The authority individuals gain over more aspect of their lives and bodies comes with more possibilities to explore them in ever more intricate detail, gathering new, deeper knowledge about the self, particularly its materiality. The self is constructed as a "database" - as Schüll demonstrates for continuous self-tracking (324) - that can be explored with technological tools and commercialized services. In some of the case studies DIY has become a means to (re)shape the engagement with one's own biology through exploration and experimentation. This new type of creative, open, inquisitive engagement creates a more intimate relation to biology and the body. This self-knowledge and self-exploration, as many of the discussions have shown, seems to be culturally connected also to ideas of self-enhancement - not just of an abstract sense of self but of a 
material, concrete body. Drawing on discourses of bodily plasticity, in cultural representations self-knowledge becomes an entry point for modifications of the physical status quo, on molar and molecular, cosmetic and curative levels. This possible modification of the body is the most prominent material consequence of many of the DIY techniques; at the same time, however, it is a deeply personal, individualized consequence because these modifications are performed by the self on the self according to self-set norms and imaginaries. These self-enhancements do not have to be drastic steps, such as integrating technology into the body to gain extra senses, but can also be more mundane such as reaching a personal feeling of wellness through vitamin supplementation. What is crucial today is that these tools are accepted as new ways to materially re-configure the body.

But the use of technologies, or more precisely information as their desired outcome, also gives rise to new modes of vulnerability: Joseph Dumit and Regula Valérie Burri maintain that today we have information "to the point of excess" (223-24). We should thus also ask ourselves if we can have too much information? Do we strive to know more about the self than is good for us? Knowledge about the body and self is often volatile in itself, it creates more uncertainty than certainty. This uncertainty leaves the self in a state of constant possibility, creates patients in waiting, leaves room for interpretation and doubt, apprehension. For example, Dickenson argues that knowledge through DTC testing and the resulting perception of the self as a "patient from cradle to grave" might actually undermine personal agency and choice (60). As Chrysanthou mentions, the availability of information may create information-related illnesses, such as "information anxiety:" The impossibility to keep up with the flux of information about health online can, for example, create further negative health outcomes such as chronic stress and anxiety - especially because the information we want to use to tame our uncertainty about our (physical) condition might be uncertain or misleading itself (473-74). Similarly, Joseph Dumit argues that the insecurity we feel about our physical status quo persists despite our increasing knowledge and research about risks and treatments, "as if the more we know, the more we fear; and the more we fear, the more preventive actions...we need to take" (1-2). A precare existence is the result. Instead of the promised control, the individual realizes that control, like perfection, is a moving target: More, and more minute, knowledge only creates further instances that need to be controlled - thus doing little more than miniaturizing the targets of control. Talking about tracking, Schumacher thus rightly contends that one of the most important questions to be asked is how we as humans deal with the abundance of knowledge about our selves without losing our humanity (51).

\section{Bodies Beyond Hacking}

Here we are also reaching the affective dimensions of DIY biology and medicine: Affective structures of hopes and promises, fears and anxieties underpin much of the DIY practices under consideration in my case studies - promises of perfection, of control, of wellbeing and comfort, promises of access and participation as much as anxiety about bodily contingency, about uncertainties, about loss of control, about death, disease and 
decay, anxieties about being overheard, neglected and mistreated. ${ }^{26}$ I would argue that the shared "affective atmosphere" (Ben Anderson, "Affective Atmospheres" 77) of hope and desire created also through DIY techniques is used to counter overarching societal moods of fear and anxiety about the contingency of (future) embodiment. In times of overwhelming precarity - in all its different shades and connotations - we follow a fantasy "of one day not being vulnerable" (McCormack and Salmenniemi 8). What is created is a promissory atmosphere, the promise of control over the previously unknown, unruly and untamable: nature, biology, the body. DIY perpetuates the hope of true selfdirectedness and self-optimization. These promises and fantasies might however fail to materialize, thus perpetuating the very vulnerability they aim to prevent.

In light of this vulnerability, we should also take a minute to look at "forms of life" (Rose, Politics of Life 105) already deemed vulnerable today - disability, old age, disease, marginalization - and ask whether these can be hacked as well. Is there a form of life that is not hackable, a life beyond hacking? Proponents of biohacking would argue that yes, especially old age is "hackable," at least if we aim at staving off the frailties associated with old age. But then, but what about the elderly today? Moreover, will improvement culture lead to new forms of eugenics and social Darwinism, in which those who cannot be hacked or opt out are deemed inferior? What happens to those who are not hackable? Or who do not want to hack themselves? Who opt out of improvement culture? These questions lead to problems of discrimination and social stratification, of obligation and a new biopolitics based on an incentive to 'hack' the self in ever more ingenious ways - a "regime of bio-making" that according to Jen might be the outcome of today's DIY practices. $^{27}$

But here we also once more see how the personal becomes political - a standard assertion of feminist inquiries and activism - how individual choices are embedded in larger cultural concerns and (bio)political considerations. DIY can exacerbate experiences of vulnerability, especially if it becomes a new requirement for participation in the neoliberal market place, an obligation to care for the self yourself because no one else will do so, or a tool used by states and institutions to enforce a bio-politics of participation. On the other hand, Delgado and Callen, for example, claim that DIY is a political action especially because it questions our "dependencies on institutions that do not serve to cope with the precarious condition of life" (180-90). DIY for them

26 For example, a promise of certainty and control drives people to test themselves, but anxiety can possibly influence the "voluntariness" of testing. In a culture that creates more and more anxiety about bodily contingency, testing becomes less voluntary and more of a way to counteract that anxiety and grasp for certainty: It becomes not an action but a re-action.

27 Jen warns of an "emergent bio-making regime." She writes: "With increased commercialization of bio-maker culture and tighter alliances with biocapitalism and the US military-industry complex, a rising bio-making regime may reconfigure neo-liberal governance in ways that newly discipline subjects to assume bio-making as a moral imperative. This would disparately impact marginalized populations who already encounter barriers to scientific literacy and skills." (136-37). As a brief historical reference - or rather a historical continuity - this element of obligation shares some characteristics with DIY home improvements in the 1950s. As I have discussed in Chapter 4, also home improvement DIY took on a coercive quality especially for men, when it became an obligation, a standard part of the regime of masculinity, to be a "handy man." 
becomes not a source of precarity but a solution: A creative intervention, as also many of the case studies have shown, that shines a light on exactly those conditions that create precarious conditions of life - dependencies, ideologies, differential distributions of resources and power - and demonstrates ways to subvert them.

\subsection{Me versus We - Social Stratification and Collaborative Individualism}

"When we work, it is with the betterment of the community in mind -- and that includes our community, your community, and the communities of people that we may never meet. ...The biopunks are actively engaged in making the world a place that everyone can understand. Come, let us research together." (Patterson n.p.)

In this quote, Meredith Patterson voices an explicit invitation, extended to everyone who heard and later on read her Biopunk Manifesto. This invitation stresses not just that satisfying curiosity should know no boundaries or barriers, that access to knowledge should extend to everyone, but also invokes a community, a 'together' of like-minded and similarly motivated people. On the first glance, this stance seems to stand in marked contrast to the focus on individualism in contemporary biomedicine and biotechnology, the consumerist, almost obsessive nature of individualist desires for health, perfection, and self-improvement, the 'yourself' that is an such an explicit part, on the most literal level, of 'do-it-yourself' techniques. The discussion here will focus on the social consequences of DIY biology and medicine, more specifically, on the interplay between individualization on the one hand and an ethos of collaboration, community and sharing on the other.

The individualism perpetuated by many DIY techniques can create negative social consequences by negating community principles and creating new forms of differentiation. I will discuss these new types of differentiation as one potential outcome of the trend towards DIY. Who is part of the trend? Who is granted access? Or, as Clare Jen put it, "[f]or whom is bio-making a democratization process? Who remains on the margins of the margins?" (134) And what are the results of this social stratification, locally and globally? These are some of the questions I will touch upon. But as many of the case studies have shown, despite the strong focus on the individual in DIY, DIY biology and medicine can, on closer inspection, be considered also as a collective approach potentially creating new opportunities to subvert, criticize and resist the consequences of the discourse of individualism. Can this collectivizing moment have broader social ramifications? Can it subvert the differential distribution of resources, tools and knowledge? A second scenario considers DIY biology and medicine's collaborative nature as a chance for social transformation.

\section{Me Trumps We}

The focus on the individual in DIY biology and medicine is visible in most of my case studies - either through the underlying rhetoric of individual responsibility, the self-directed nature of the DIY endeavors or through the focus on the self and body as source 
for (molecularized) self-knowledge and biological manipulation. Self-help books, for example, aim at individual optimization, the discourses created by genetic and molecular testing companies explicitly focus on 'yourself' as the object of inquiry, and also Wohlsen's narrativization of Biopunk takes as its focus individual biohackers and possibilities for self-enhancement more often than not. This individualism is especially visible in the discussions of personalized medicine and digital health technologies, but it is also the cultural foundation for DIY in the larger sense. Donna Dickenson argues that "Me medicine" has superseded "We medicine" as the dominant frame in healthcare. More precisely, she is concerned that the current focus on "Me Medicine" - those "promissory technologies" that characterize debates around personalized medicine, such as directto-consumer genetic testing, pharmacogenomics, private umbilical cord blood banking or enhancement techniques $(1 ; 144)$ - is surpassing "We Medicine," public health measures for example. This shift would cause the American "cult of individualism" to undermine the notion that medicine and biotechnology should serve the greater good $(2-3) .^{28}$

During the past centuries, these individualist tendencies in the US have increased: Robert Putnam, in his book Bowling Alone, describes how the "heathy tendencies of Americans to make connections and form associations," especially between people from different backgrounds and social situations that would otherwise not interact with each other, has been declining in the late $20^{\text {th }}$ century (cf. Gauntlett 137). Economic forces, constant competition and a neoliberal ideology of personal freedom have made American society an increasingly individualistic one. If we are concerned only with individual access, individual modification, individual preferences and individual freedoms, however, we lose sight for broader concerns. It is problematic if one of the highest values of a society is individualism because that risks negating social factors, structural problems and inequalities. It is an ethical concern as much as a social, practical, and indeed, in the case of biology and the body, a potentially material one. A focus on the individual blinds us for the collective ramifications. As Lupton claims for self-tracking, the prevalent notion of autonomous individualism means that communality, situatedness in social, political, cultural contexts, participation in social groups, acculturation to certain norms and values, and relationality are often not truly recognized (Quantified Self 140). Extreme personalization erodes possibilities for community action and empathy and negates that we as humans are always situated in and shaped by what and who is around us. One example that Peter Conrad discussed already in 2007 are biomedical enhancements: Through their focus on individualist thinking (and individual dissatisfactions) they do not question social standards or expectations on a collective level, he argues. Instead, they risk decreasing the diversity of a society and might bring to the forefront (ethical/moral) conflicts between individual benefits and the public good (Medicalization 91-96). Likewise, Lemke claims that genetic diagnosis creates a dynamic in which optimization of individuals takes precedence over the transformation of (dis-

28 Dickenson looks at "threat and contamination," "narcissism and the "bowling alone' phenomenon," "corporate interest and political neoliberalism," "choice and autonomy" as factors that potentially influence the rise of Me Medicine and decline of We Medicine (10-29). 
criminating) social contexts: Social progress, he argues, is replaced with a "therapeutic regime of self-improvement" ("Disposition" 558).

Of course, such concerns are also applicable on a larger scale, for example in relation to health care: While individualism and personalization can lead to more cost efficiency, fewer side effects and better targeted therapies, improved quality of life for individuals (Sharon 99), it also comes with tremendous 'side effects.' Stevens writes that one of the problems with the vision of individual autonomy and personal responsibility in healthcare is that it changes the public's view of health care from a social, public, collective responsibility to an individual one in which the individual has to take care of their own well-being: The result is a lack of concern for notions of care - and care work, I would suggest - and the collective responsibility in a community, threatening the "notion of health as a public or social good" (Stevens 308). As Rose writes: "If our medical 'imaginary'-our image of what healthcare should be-is 'all about me' it may well have serious consequences for my willingness to pay for, let alone care about, the health of others who are not related to me-not just others in my town or my country, but others in far away and distant places." ("Personalized Medicine" 350-51) Instead, such individualization replaces the notion that the state cares for its citizens with a discourse of individual responsibility (and the resulting possibilities for blame), thereby turning from welfare as a public and collective endeavor to an individualized, neoliberal model of governance (Sharon 99). As a result, the utopian body project that Chrysanthou talks about - a healthy body - might create an unhealthy society as the individualization and privatization of health promotion foreclose social, political, and holistic strategies (447). The "rush" towards personalization, individualization, preventive-preemption might divert resources from those health care interventions that are acutely needed and/or have proven effective in the past, those "impersonal, population wide, solidaristic social interventions" that have improved health for all, such as clean air and water, sewage, food control and population-wide vaccination (Rose, "Personalized Medicine" 348, 350-51). The promise of individualism, thus, might turn out to be a burden after all.

\section{Privileged Bodies}

The creation of "privileged bodies" is one possible consequence of the focus on individual solutions and the loss of collective responsibility. "The body-in-isolation is, of course, a privileged body," writes Victoria Pitts. What she refers to with this "body-in-isolation" is exactly the individualized body perpetuated also in DIY culture: the body standing apart from social, economic, political, personal ties, characterized by a 'right' to individuality, to standing alone, negotiating to get one's own, self-defined needs met through technological access." This body, usually in Western cultures, is flexible, a project, a "body of privilege" (Pitts 191-97). While this privilege is manifold - from physical location, to access, to economic situation - I first want to consider this privilege mainly in its material form, its connection to enhancements.

Especially due to the inherent (social and cultural) connection to some form of optimization, DIY also carries with it the potential of furthering the creation of physically privileged bodies that seems to be an inherent fantasy of biotechnologies today. In the neoliberal need for self-optimization, it is no wonder that also for DIY biology and 
medicine the surveillance and modification of the self is a common goal. Even the developers of open-source tools like OpenPCR, we have seen, relate DIY biology to a more personal engagement with one's own biology, a familiarization with and monitoring of the previously hidden, material recesses of the self. Might the liberalization of access to biomedical tools, technologies and knowledge actually help perpetuate improvement as a necessity? If the promise of democratization is fulfilled and 'everyone' has access to the technologies of enhancement, there would be even less of an excuse not to use them, at least if you use them in socially desirable ways. A culturally entrenched coercion towards self-improvements could be the result.

DIY improvements potentially create a new type of embodiment, an embodiment of possibilities and openness, highly flexible and modifiable. But modification also always carries with it risks of coercion, exclusion and differentiation: To quote Samuel's journalistic take on biohacking's risks: "What if biohackers' 'upgrades' don't get distributed evenly across the human population?" ("How Biohackers") Will we have new types of exclusion and differentiation, between those enhanced/modified and those who are not? In this scenario, some bodies are made 'better' than others, who get stigmatized, discounted, disvalued. Those who do modify, or who have access to modifications, are privileged through their access and, more substantially, create privileged bodies through their ability to potentially shift their biological materiality according to their personal needs and those of the market. ${ }^{29}$ In contrast to its promise of democratization, DIY technologies could morph into privileged, to a certain extent exclusive (bio)technologies that often do not recognize or fail to consider questions of social stratification and intersectionality.

\section{Differentiation and Segregation}

The privileging of some experiences and bodies as well as the concomitant differentiation of society, might in the long run lead to a new form of medical or biological segregation, a type of new, culturally sanctioned discrimination. Here, we have a classical differentiation into who is allowed - or here empowere ${ }^{30}$ - to participate, who is heard in public debates, whose experiences are denied and whose experiences are included. According to Keulartz and van den Belt, the notion that the "public" in DIY is necessary active, involved, engaged disenfranchises those who are less active, those who are less able to do things because of unequal access to time or resources, those who are physically unable to take part, those who refuse (for moral, cultural or other reasons) to take part (13-14). I want to look at this medical or biological segregation on three different levels: the molecular, the local and the global.

29 Tellingly, Samuel goes on as follows: "Here's another risk associated with biohacking, one I think is even more serious: By making ourselves smarter and stronger and potentially even immortal (a difference of kind, not just of degree), we may create a society in which everyone feels pressure to alter their biology - even if they don't want to. ...In a world of superhumans, it may become increasingly hard to stay 'merely' human." ("How Biohackers" n.p.)

30 Empowered might be a better term here because of the aim of active empowerment through DIY - the idea that the movement actively furthers the participation of lay people, thus potentially also determining who is allowed to participate. 
On the molecular level, new forms of molecular differentiation mean that prejudices and discrimination are not necessarily based on gender or race/ethnicity but other criteria related to the body - criteria that today are in constant flux. Not only categories such as 'normal' or 'deviant' are in perpetual negotiation. An unpredictable shift to new criteria could mean that all social groups might get marginalized as soon as new scientific knowledge or aggregate data find them deviating from the 'norm. ${ }^{31}$ Moreover, these new criteria that can potentially be used to divide society into groups are today often located in the body, not visible to the self without the help of technology. For example, the informational body created by (DIY) biomedical testing creates new types of differentiation or racism that is not based on "skin- [or surface-]morphology" but based on "information," a very abstract, non-tangible and invisible basis, that, however, is perceived to be intrinsically linked to identity simply due to its "depth," its hidden nature and its omnipresence in the discursive construction of bodies as information (cf. Clough 223). Data or information here form the basis for distinctions into groups with some deemed superior and some inferior or at risk. As one potential outcome of such practices, genetic engineering could give rise to new types of human enhancement that builds inequalities literally into the biological make-up of humans, creating a more divided society and world. Democracies, as Francis Fukuyama argues, however, are built on the idea of equality, of all beings being created equal. Inequality inscribed into our DNA through enhancements could, thus, undermine democratic principles and human rights. According to Stevens, the outcome of such genetic engineering would be a "radically unequal and divided society" (48). We might not even have to think that far because either on their own or as precursors to other, more radical practices also some of the DIY techniques carry with them seeds of division and inequality: A differentiation into groups based on the molecular make-up of and information produced by the body.

On the local level, we come closer to those categories often associated with questions of discrimination: disability, class, social status, gender, race. Here, differential distributions of access can create new forms of segregation. Physical and cognitive disor rather inability, for example, are exclusion criteria for participation in a highly active, knowledge-based ideology such as DIY. ${ }^{32}$ Moreover, the economic situation of an individual, especially in consumerist DIY, will decide whether or not they can take part in as there is no 'standard sequence.' For the kilogram or time, society has agreed on a physical 'standard' to define weight and time. Such a standard can never be set for the genome, which directly impacts questions of 'normality' and 'disease.'

32 As Keulartz and van den Belt have claimed the ideology of active participation also disenfranchises those who are simply not able to participate - physically or mentally. The cooperation of makers and people with disabilities could be read as a positive example of how this type of differentiation can be overcome through the production of competence and expertise in community, the recognition that people with disabilities are experts on their own (individual) embodiment that need to be heard and included when developing new aids and tools. Still, the fact remains that sheer physical or mental difference can easily lead to the exclusion of people with disabilities from the promise of do-it-yourself. Moreover, self-optimizations as they are perpetuated by DIY biology and medicine have a hyper-able subject as the desired and promised outcome - furthering instead of questioning the dominance of an ableist culture. 
the narrative and promise of democratization. Already today, a high degree of disposable income is needed to afford the tools and technologies of DIY biology and medicine, which is reflected in the current make-up of the movement: At the moment, DIY biology and medicine is still a very much White, middle-class phenomenon for people with higher degrees of education and income. ${ }^{33}$ While more development might make technologies cheaper, chances are high that self-knowledge and self-enhancement in a consumerist society will always come with a price tag. DIY biology here might continue a division in society, where those with higher social status already today have better access to health care, self-knowledge and (medical) interventions. Biopolitical governance is no exception, as it often (re)produces racism and differentiation. Many people are "denied the right to health" and cannot make decisions about their health: "their biological selves are precarious and exposed to illness, injury, death - unavailable for preemptive intervention and optimization" (Krupar and Ehlers 4). Racism, Daniele Lorenzini recounts (following Foucault), is a way to differentiate populations, "the biological continuum," and create hierarchies between different human groups - some of which will be exposed to risks of death more readily than others (43-44). Differential distributions of value, life chances and vulnerability, thus, are an inherent part of biopolitics and biopolitical governance. According to Krupar and Ehlers, the result is a (deliberate or not) logic of "let die" in which some parts of the population are harmed to protect others. The lines between those groups are sometimes blatantly obvious, but most of the time less so, reproduced by the biopolitical system itself. Practices that "foster life" can at the same time devalue other lives, especially those "at the peripheries of 'ideal' citizen/subjecthood" (Krupar and Ehlers 4, 12-13). These differential valuations, of course, affect disabled people and Black, Indigenous, People of Color (BIPoc) more often than others.

In addition, gender can become a source of social stratification if we talk about limits to access. Historically, DIY practices have often been divided along gendered lines. Looking at contemporary "bio-making's" gendered dimensions, Clare Jen argues that scholarship should also consider classed and gendered barriers to DIY participation, such as educational barriers, the economic situation, surplus time apart from caregiver responsibilities, or unwelcoming community labs (127). She claims that often the narratives, spheres and mythologies of bio-making are male-centered, discounting female biohackers, their contributions and the long history of systematic exclusion from scientific participation. In fact, also many of the protagonists I encountered during my research were male - it is certainly noteworthy how, for example Wohlsen, describes female biohackers in different terms than their male counterparts. ${ }^{34}$ Such representa-

33 For example, Lupton writes that owners of wearable devices are more likely to have a higher household income (Quantified Self 31). The affluent and those who can buy into new developments in healthcare have better access to medical care and could, with the increase in personal health technologies, also have better access to those, leading to a widening gap in healthcare (N. J. Fox 149). A 2006 Report on Human Enhancement (2006) similarly ascertains that more affluent members of society gain access to new technologies, also health technologies and human enhancements, sooner than others, increasing their advantage (Williams 9-10).

34 For example, in Wohlsen's Biopunk Kay Aull seems to have to measure up to traditional images of scientists as male: "Aull is tall and beanpole skinny. She carries herself with the stiff awkwardness 
tions, or under-representations, might shape the whole movement going further, leading to a perpetuation instead of a transformation of those traditional exclusions. In contrast to the dominant, male-centered mythodologies Jen argues that "feminist biohealth hackers" have contributed a great deal to the movement, citing examples such as Kay Aull and Meredith Patterson (135).

The global dimensions of DIY biology and medicine and its possibility for segregation warrant closer attention for many reasons: DIY is inherently privileged in the US, people opt out of a system by choice, while in other countries DIY might be a necessity simply to be able to take part in biomedical innovations (that save lives); the DIY movement itself has discovered global questions as a valuable concern, and, lastly, the question of access is much more pressing on a global scale. Especially when we look at DIY biology's newfound interest in global questions of resource distribution and access - with its concomitant projects that try to amend some of the more pressing concerns - we soon realize that despite of good intentions these practices can also be deeply problematic.

For one, "privileged bodies," as of today, are primarily located in the Western hemisphere: They are the ones benefiting from technological innovation for their own life projects, for them DIY is fun, a hobby. ${ }^{35}$ Especially when DIY is used for enhancement and optimization, it is not an equalizer but a differentiating force: Underprivileged people(s), in the current frame of DIY biology, might become even more disenfranchised, widening the gaps that already exist today, in relation to 'biological' categories like life expectancy, mortality, vulnerability, but also levels of education, employment rates, household incomes, and so forth. In large parts of the world, what we term DIY is an (economic) necessity. Where access to high-priced but potentially lifesaving technologies is missing, DIY solutions might be the most feasible way of granting access to these technologies, as the example of the LavaAmp hack demonstrates (cf. Case Study IX). One could argue that any tool is better than none, especially if they are functioning well; that sometimes the cheaper but ingenious solution is making the best use for the most people out of limited resources. However, allocating DIY solutions to low- and middle-income countries as a replacement for the 'real thing' perpetuates problematic distinctions and associations. Should we accept DIY's make-shift, cheaper, and 'justas-good' solutions as the answer to global problems of resource distribution? DIY here might become an easy-way-out that avoids large scale, controversial - and for large parts of the world also painful - reflections and discussions about privilege, entitlement and the worth of human life. In the global perspective more than the local one we thus also touch upon questions of distributive justice. How and where biotechnologies can be used highlights how resources, benefits and burdens are divided unequally between

of an adolescent boy still not used to his overlong limb." (Wohlsen 9). Why is Wohlsen comparing her to an "adolescent boy" or why is her marital status of more importance than in her male cobiohackers' descriptions? Even if done subconsciously, Wohlsen seems to treat his male and female interviewees slightly different.

35 What should be noted as well is that DIY biohackers in countries like the US do have a functioning medical system to fall back on, for example if they harm themselves in the process. When such a comprehensive medical system is missing, people are even more vulnerable. 
peoples and nations. This of course, is also a moral and philosophical question concerned amongst others with social justice and collaboration on an international scale: What is needed to live a dignified life and who has the responsibility to facilitate that (cf. Nussbaum), how can we ensure that a right to bodily integrity and health (prior to potential optimizations) is guaranteed for everyone, how do resources have to be redistributed to reach a state of equality and equal opportunity around the globe? Or more to the point: For example, the often-claimed access for all would require large scale literacy and education efforts, redistributions of wealth (to allow people to buy into DIY practices) and technologies (to provide the tools), if it were aimed at a global level. These requirements are not met on a national level and even less on a global one. ${ }^{36}$

Moreover, because DIY biology as a cultural phenomenon has emerged primarily in the Western world and large American and European cities, as Meyer writes ("Build" n.p.), their subsequent spread to other parts of the world could be seen as a new form of American exceptionalism, where American definitions of health, of ideal bodies and lives influence cultures around the globe - and this is especially true for commercialized solutions. As we have seen, numbers, norms, data and technologies shape bodies and experiences of embodiment, and those might not necessarily be built with diversity in mind. Rather, they reflect the worldview, environment and prejudices of their producers, which carries with it tremendous potential for discrimination. If tools are produced by Western biohackers for people in low- and middle-income countries, we also risk perpetuating a new form of technological colonialism in which Western hackers are portrayed as the heroes and saviors. Instead of strengthening local solutions, of empowering local amateurs, makers, hackers and 'need-knowers,' DIY technologies might be exported by the West. This of course is an inherently negative future trajectory that perpetuates outdated differentiations, negates the competence of other peoples, and buys into an American myth of (moral) superiority and virtue - taking away much of the diversity, creativity and chances provided by DIY.

On the other hand, the opposite might become true as well: DIY and making do take different forms in different countries. Ideally, through DIY local problems can be addressed using a new epistemological practice - local solutions for local problems, arrived at through an epistemology based on participation, experimentation and creativity. Jen's feminist analysis of biohacking practices touches upon such considerations. She argues that alternatives to biomedicalization were often constructed by feminist bio-health hackers out of necessity and the realization that they could cater to their own needs better than the establishment (135). ${ }^{37}$ Today, this feminist impulse, Jen claims, is visible also in biohacking efforts that replicate PAP smears, a common gynecological diagnostic tool, with vinegar in Thailand (McNeil). ${ }^{38}$ If we consider access to healthcare and ed-

36 Of course, such question cannot be answered in this short discussion of DIY's common themes, potentials and downsides. I still want to raise them to point out larger (even though future) considerations and potential outcomes of some of the DIY practices today.

37 Jen includes women's self-help groups as examples: "Pelvic self-exams, using speculums and mirrors, gained practical and iconic significance as an empowerment tool and technology of resistance." (135)

38 This "hack" was developed at John Hopkins medical school. Household vinegar can be used as a cheap tool to find and treat pre-cancerous cells, McNeil explains, by simply brushing it on a 
ucation as a universal right, DIY solutions could unfold a transformative potential that allows people access to both cheap and effective healthcare solutions as well as (science) education to empower local replicators to create their own solutions. People are empowered to create their own solutions because they know what they need and how they need it. DIY and the maker movement, with its possibilities of small-scale and localized production, provide the tools and technologies to make those visions a reality, with the downside of the as of now high costs associated also with DIY. When knowledge is transferred - on an equal basis - the transformative potential of DIY in low-income countries might even be bigger.

In summary, today we do not necessarily find the access touted by DIY biologists. As is visible in the introductory quote for this section, Patterson likewise seems to misjudge the exclusiveness of her own community, driven by ideals that as of now still need to be fulfilled. Intersectional considerations would also be interesting, as the convergences of class, gender, (dis)ability and race/ethnicity might shine a new light on exclusions from DIY's promise. But first we need to recognize DIY as the privilege it is, at least in the form it is done in most of my examples, as a conscious dissociation from the system, a voluntary outsider status. A global perspective on the differentiations produced by DIY biology necessarily also includes broader questions of resource distribution, human rights, and equality. DIY could here be seen as perpetuating the underlying problems of unequal distributions while at the same time providing a possible solution by making the technologies available in the first place. It is, however, on a global scale that I would see the largest downsides to DIY's democratization narrative - from which large parts of the global population are excluded anyway for the simple reason of lack of access to education and capital to buy into its promise.

\section{Collaborative Individualism}

So far, this discussion has focused on the negative effects of the individualism that carries DIY practices. But, as many of my case studies have shown, DIY can also be a collaborative effort. Often it is not just the individualism one might expect from DIY techniques that is performed but an interplay of both individualization and collaboration. I would argue that the forms that DIY takes today, in the sectors that I am looking at, manage to integrate both: they combine an individualist, project-centered view with community integration and collaborative inquiry. More so, they depend on each other for success. ${ }^{39}$

We can here take inspiration, once more, from Nikolas Rose and his work on "biological citizenship" with Carlos Novas. For them, biological citizenship is both individ-

woman's cervix. Pre-cancerous cells turn white and can be treated "frozen off with a metal probe cooled by a tank of carbon dioxide, available from any Coca-Cola bottling plant" (McNeil).

39 Individual projects rely on collective knowledge and sharing, for example, by making use of tutorials or advice groups, using the wisdom of the many to find a suitable approach. At the same time, often collective projects also depend on individuals to take part and refine them: For example, large scale DIY or commercial genomic studies rely on individuals to share their data for a common cause; large scale citizen science efforts rely on individual contributions of time and knowledge. 
ualizing - the somatic self and personal responsibilities - and collectivizing through the creation of new forms of "biosociality" (Rabinow), creating new collectivities around biomedical classifications (441-42). This duality is also visible in DIY. Genetic and molecular testing, for example, is based on individualization, personalization and self-knowledge, but it is also inherently relational. Similarly, in many efforts of biomedical tinkering with technology knowledge and literacy are produced through collaboration and community. We experience a growth of new forms of community, new forms of solidarity and social networking in spite of the pervasive rhetoric of personalization (Rose, "Personalized Medicine" 350-51). These include patient and advocacy groups - such as the Open Diabetes Community - and other groups organized around new types of knowledge and new molecular identities and experiences of embodiment (cf. Pavone and Goven 13-14). This new community of practitioners demands new rights to access, participation and knowledge. It is a form of activism similar to those older forms of biosociality. "Making is connecting" is the basic formula that Gauntlett finds for this phenomenon: connecting things, connecting ideas, connecting people (2).

The internet plays a crucial role for this process of community formation in DIY biology and medicine. As a space of exchange, community and empowerment, it provides fertile ground for the formation of not just physical meet-ups but also "virtual communities" (Csordas 178). The boundaries of and membership in these virtual communities, according to Thomas Csordas, are fluid, which makes them perfect tools for collaboration among groups as well as a project-oriented creation of teams with shifting competencies. But those new collectivities are also created and fostered through the media and discourses: Cultural representations work as collectivizing instances that produce community. DIY biology and medicine's community-oriented nature, therefore, satisfies not just personal scientific curiosity but also an inherent human need for social connection, as I have also discerned in Case Study V. We can borrow aspects of Benedict Anderson's concept of "imagined communities" to describe the communities that arise out of DIY biology: Even though many of their members will never meet each other, they nonetheless feel a deep connection, "in the minds of each lives the image of their communion" (6). This (imagined) connection is based on similar interests, similar values or similar experiences - depending on the type of community. On different levels, science becomes a collective project, a social-scientific utopia in which all stakeholders participate. This collectivity allows for a trickle-down effect that is the basis of DIY's educational potential: Knowledge is disseminated from those who have it to those who lack it or are currently excluded from it, for example through the structural and personal connections between DIY biology and institutionalized science. As a collective project, DIY biology and medicine also is turned into a form of 'do-it-with-others' (DIWO), or 'do-it-together' (DIT), new acronyms used to denote collaborative forms of DIY that seem to transcend the individual.

However, the knowledge that is produced in DIY biology and medicine, collaboratively, following scientific standards, is used on individual bodies for individual purposes. What we find here is, what I term a collaborative individualism in which people come together to do things individually, in which collectivity and individuality are in constant interplay. While sometimes either one may dominate, they are never really dialectically opposed. Meyer borrows the term "connected individualism" (Flichy) to de- 
note this inherent connection between the individual and the collective, "halfway between individual practices and group practices, between the logics of autonomy and the logics of networks." For him, this connection is visible in how people might perform biology autonomously in their own laboratories but still rely on the "collectives of people, ideas and objects" formed by DIY biology, the new biotech economies he describes (Meyer, Domesticating 19). If we want to stick to the designation 'DIY' - due to its cultural purchase or historical continuities - it can be useful to rethink the 'yourself' not just as the active individual doing biology but as capturing this individual through its inherent relations to the network of other practitioners and institutions (in the broadest sense). Donna Dickenson also acknowledges that "We" is alive and well in biomedicine in certain instances, that the choice to be made does not necessarily have to be between Me and We but that we can also find a way in-between, combining both.

This collaborative nature of DIY biology and medicine can balance some of the concerns connected with individualism: It creates community, instead of erasing it, social questions and relations become part of the equation, and it creates empathy and cohesion. But is this collectivizing moment enough to transform a broader culture shaped so much by individualist thinking? We need to keep in mind that this newly-minted community is still apart from the rest of society, it is still a rather closed-off group of people. In this community, individualism and collectivism are in a constant interplay of competing interests and approaches as well. In the end, we are left with a paradox of individualism and community, that as of today cannot be resolved. Is the collective nature lived reality or merely a promise, a utopia? 



\section{Conclusion: Between Politics and Promise}

"In the same way, the real significance of DIY biotechnologies might lie not in any particular technological achievement but in the provocative questions they raise. They may never cure cancer. Yet their idealism and critique of the scientific establishment could make a lasting impact. In reality, American DIY movements have rarely been about dropping out of society but about clever reimaginings of social norms. Biopunks do not build their lab tools to maintain some kind of purist separation from the existing system. They want to force the conversation about how that system works and who it serves." (Wohlsen 120)

"The ultimate DIY project becomes the body itself." (Chrysanthou 471)

For Wohlsen one of the fundamental aspirations of DIY biotechnologies seems to lie in their critical nature: They raise questions, provoke thought, start conversations, open science. Through its tales of biohacking in practice, Wohlsen's book is part of this quest. Only when DIY biology is talked about, inserted into public discussion, represented and debated can it live up to its potential. This potential lies not in technological innovations but in culture and society - in its political weight. It attempts to shift social relations and scales of power, to create new ideologies of participation and epistemologies based on fun and experimentation. Most of the techniques of DIY biology and medicine are "boundary-blurring practices" (Ratto and Boler 18) that bridge fields considered as oppositional and bring them into conversation: collective/individualistic, nature/culture, private/public, open/closed or proprietary, top-down/bottom-up, inclusion/exclusion, lay/expert. As Ratto and Boler argue: "DIY practices ideally create [a] 'maneuvering' space," in which we can "rethink binary distinctions" (18). This "maneuvering space" 
opens up possibilities of intervention and transformation into existing structures of knowledge production, outdated systems and the status quo; differential distributions of power and resources; the minute particles of life and the flesh and blood of the human body. In DIY biology and medicine individuals become agentic subjects and objects of intervention at the same time. DIY biology, following Meyer's train of thought, is both a continuation of the "involvement of amateurs and citizens" in science and a rupture because now people do not only want to observe and describe but experiment with and (re)engineer the biological world ("Hacking" n.p.). This 'tinkering' with the biological world extends towards the human body itself - in many of the technologies the (material) body indeed becomes the "ultimate DIY project" (Chrysanthou 471).

\section{Four Strategies of DIY}

On the basis of my analysis and discussion, I want to delineate four, often interwoven, strategies of how DIY is employed and represented in biology and culture. The most cited and probably most influential strategy is that of a symbolic force or creative intervention. However, many commercial forms, and those with a higher prevalence in society, in fact are far from being creative interventions. Instead, they can be regarded as coping mechanisms in a world of uncertainty and vulnerability, a requirement in an emergent regime of biomaking or a marketing strategy employed by start-ups and medical companies. These strategies exist alongside each other, can vary according to the practitioner and context in which they are used.

By many scholars, DIY biology is seen as part of a whole array of interventions with similar ideologies: It is not an "isolated phenomenon" but part of a conglomeration of DIY initiatives in different domains (Keulartz and van den Belt 16). According to Delfanti, "transparency, sharing, distributed creativity, peer production, and distrust for institutions and bureaucracies are becoming more and more widespread" today, examples of which can be found in the hacker network Anonymous, the leaking platform WikiLeaks, the maker movement, or the Pirate Parties in Northern European countries (Biohackers 138). ${ }^{1}$ DIY approaches to biology and medical technologies can be part of this shifting value system, especially in their guise of creative intervention. This strategy is mainly visible in educational tools, open-source programs and technologies, inclusive projects and patient advocacy, but also in more extreme bodyhacks and niche phenomena, such as implantable (medical) devices or DIY medications, which often have a high potential for polarization. Here, DIY techniques work as demonstrations: they demonstrate the feasibility of (new) technologies, that different forms of arriving at knowledge are possible, that systems of power can be restructured, that things can be done otherwise, that they can be done better, cheaper, more accessible. As "vectors 'from below" these demonstrations "introduce doubt and controversy" (Rose and Novas 44647), working against the system, dependencies, capitalism, subordination. According to Delfanti, DIY biology's success is based on this "symbolic power" and quality: "DIYbio, 
making biology hackable in all these different ways, is producing the picture of a different way of conducting research in the life sciences: more open, horizontal, within a very mixed constellation of different actors such as start-ups, universities, individuals and community spaces, with a prominence of small and open companies instead of the slow giants of Big Bio." (Delfanti, Biohackers 126) These creative interventions could, in the end, also create a new culture of science. For Delfanti, scientific cultures can and should be updated with new cultural elements and norms: The hacking of biology, thus, for him is also an "innovative recombination of cultural elements" and science (Biohackers 16, 130). This recombination of different elements and ideologies has the potential to create "a novel, alternative paradigm of knowledge production outside of the academia and industry walls" (Keulartz and van den Belt 2). DIY biology and medicine here takes on its guise as a social movement, it is a type of "critical making" (Richterich). But beyond this symbolic interference in systems and structures, it is also symbolic on a more personal scale - opening new ways of engaging with the body, getting to know one's biology, optimizing it where desired.

As symbolic force and (political) demonstration, DIY biology and medicine can generate conversations. Especially those more controversial hacks attract attention: They can raise questions, overstate and exaggerate to inspire wider debates about technological possibilities and how they can or should be used. DIY, thus, opens the possibility for wider cultural and political dialogue about biotechnologies and biology (Stevens 355). In her discussion of DIY democracy, Caroline C. Lee argues that the process of public engagement and citizen participation, of dialogue and deliberation, is also a means of managing and slowing down the "breakneck pace" of contemporary life and social change (cf. 229). Similarly, taking part in science and technology, doing it oneself or together, can be a means to connect with and understand what is happening, to alleviate the uncertainty, fear, uneasiness that comes with change. To 'do-it-yourself' can mean to put on the brakes and individually, and collectively, evaluate what is done, how and why - and to then make the conversations that need to be held about future and current applications of biological technologies more inclusive. Meyer quotes one of the founders of the biolab La Paillasseis in Paris, who claims that one political aim of DIY biology is to be a "counter-power" to participate in societal choices ("Build" n.p.). ${ }^{2}$ DIY biology, in this case, is configured as a "public resource" (Gruber 3 ). The cultural discussions about the norms and ethics that guide our relationship to technology are shaped by accepted and new values, accepted and new approaches as well the possible contradictions between them. This transformative potential points to the connection between technology and culture: It is not a technological but a cultural revolution because it shapes inherently social, political, cultural questions and core values such as access, property, power, knowledge and creativity (Ledford 650; Delfanti, Biohackers 12). Their symbolic power is also what remains when DIY biology fails to deliver on its promises, when DIY hacks are not used or prove unfeasible. Krupar and Ehlers argue that biocultural studies, like the one presented here, must also work towards pointing out "alternative biofutures," in which neoliberal modes of governance are resisted (14): As creative interventions, 
practices of DIY biology point to such alternative futures and alternative ways of being and perceiving the world.

The cultural transformations, on the other hand, might also turn out to be in stark contrast to this ideal: a new "bio-making regime" in which biohacking the self becomes a "moral imperative" and tool for neoliberal governance (Jen). As much as they can be liberating, also "alternative body knowledges" (Krupar and Ehlers 3-4) such as DIY technologies can become a constraint, subjecting people to an ideology of active participation, self-education and self-transformation. Especially more mundane DIY biology and medicine techniques often are an expected form of self-care, a requirement. In many cases those are more commercial and widely known forms such as genetic testing, selftracking, exercise, nutritional 'hacks' and the like, but also self-education, the ideology of DIY insinuates, is needed to assert one's status as a biological citizen. To be a prudent citizen means to hack and (re-)make the body, to use the tools now available, to engage in the discussion around (new) biotechnologies - to be involved, engaged, curious and flexible. In our neoliberal market economy, people have to invest into their body in order to reap the benefits. These investments can take multiple forms from membership in fitness studios, to costs for surgeries, to expenses for tests, supplements and equipment, to time and effort.

This body-in-progress borders on coercion: Investment and enhancement are not only an option but a responsibility if one wants to ensure future competitiveness in the global marketplace. We need to be willing to "replace our identities with market-driven, slightly altered, and improved ideals of ourselves" (Wegenstein, Cosmetic Gaze 125). If we do not want to become 'obsolete,' we need to enhance our self, our performance and our body. This strategy is also related to one of the starting places of biohacking's cultural ubiquity: Silicon Valley and its culture of competitiveness, high achievement and high performance. Under the guise of authority and agency, foundational American values such as self-reliance, initiative, self-directedness are taken to new extremes, subjecting people under a new cultural politics of life. While, as creative interventions, the same techniques are understood as tools of empowerment, in this strategy they become tools of governance, not of top-down approaches by states or regulatory bodies but divided and distributed forms of peer and self-governance. In a new form of "sousveillance" (Lupton, Quantified Self 61) people monitor the self and others. The result of this strategy of DIY, as we have seen in the previous discussion, is a neglect of social and structural factors in favor of personalized responsibility as well as new types of discrimination, devaluation and unjust distributions of technological resources.

While DIY as a requirement implies a top-down or peer-controlled authority, individually those same technologies can be used to cope with the realities and vulnerabilities of contemporary life. For example, for some practitioners, DIY biology and medicine meets a desire for community, interaction, and social relationships in a highly individualized society: "The biohacking community also offers just that: community. It gives people a chance to explore unconventional ideas in a non-hierarchical setting, and to refashion the feeling of being outside the norm into a cool identity." (Samuel, "How Biohackers") It can become a way to assert a new 'cool' identity as biohacker, a member of a community of like-minded individuals. For others, DIY biology and medicine - and the information extracted through its use - can be used to establish their biological 
individuality. The biological knowledge gained for example through genetic testing can provide individuals with something tangible, though invisible through which they can assert their identity: "This is me; I am unique." Asserting the self through its biology can be empowering, especially in situations where people previously felt out of control, overheard, or neglected. At the same time, this individual identity exemplifies a radical situatedness in a community of biological beings, made-up from code and matter, same but different.

Additionally, more extreme forms such as grinding, but also commercial forms like tracking, DTC testing, pills and supplements - all often aimed at self-knowledge and self-enhancement - can be a strategy to come to terms with human frailty and mortality more generally. These technologies are used to counter biological vulnerability, social precarity, or diffuse feelings of helplessness, to fulfill a deep-seated desire for control and optimization. As Sigal Samuel writes, many people participate in biohacking out of a "desire to feel better - and to see just how far we can push the human body." However, when used as a coping mechanism, there really is no (natural) end point to biohacking. Or as Sigal Samuel explains to his readers, the goals of biohacking tend to "escalate:" "Once you've determined (or think you've determined) that there are concrete 'hacks' you can use by yourself right now to go from sick to healthy, or healthy to enhanced, you start to think: Well, why stop there? Why not shoot for peak performance? Why not try to live forever? What starts as a simple wish to be free from pain can snowball into self-improvement on steroids" (Samuel, "How Biohackers"). Here, we once more encounter the problem of the free-floating and moving target of perfection: Where do we stop our crusade to know more, control more, enhance more, when we always find new targets for interventions? As technologies advance, more areas of life will become even more uncertain - providing even more targets for the promise of extension and enhancement.

Lastly, in its assemblage with consumer culture 'biohacking' has become a marketing buzzword and pop-cultural obsession, used to sell unregulated products or "repackage old products, such as coffee with butter" (Hamblin). Pharmaceutical and medical companies, but also small start-ups, use this cultural hype for economic exploitation: 'biohacking' as a label is used to sell lifestyle products - supplements, nootropics, tests, the services of the new "experts of the soma" (Rose). Old techniques and technologies are reframed as hacks to monetize a trend. As a marketing strategy DIY biology and medicine is not just fully integrated into consumer culture and the dictate of the market, but it actively takes part in the formation of new "economies of vitality" (Rose) around the selfdirected intervention into biological systems and the human body. But if hacking today is such a fashionable term and practice, where does that leave its 'real' potential and transformative ideas? Does this reframing subvert hackings potential to undermine dependencies and abolish exclusions? How can we even distinguish between 'real' hacking and its 'in vogue' cousins? As the discussion in Chapter 11 has shown, consumer culture also offers great potential for DIY biology and its promise of access, if done right. 


\section{Promissory Narrative}

What these different strategies share is that at their core they combine a mythology of American individualism with biotechnologies' promissory narrative and fantasies of self-knowledge, self-control, and self-enhancement. Fantasy, promise, imagination these are also central factors in the cultural representation of DIY biology and medicine and might as such also impede on its real-life practices. DIY biology and medicine is deeply embedded in the "promissory discourse" surrounding biotech and biomedicine more generally, a hope for scientific solutions and the promise of science's absolute power. For Jen, "promissory narratives...generate future-oriented abstractions in the form of expectations, hopes, and promises." Discourses around bio-making, she argues, generate this type of positive anticipation by promising a future that is "more democratic than the present" (128). Similarly, Aguiton and Tocchetti describe DIY biology as a "promissory socio-technical vision" (829) of a democratized science with access for all. DIY biology and medicine, we can see here, is also a (political) promise: a promise of access, of democratization, of empowerment, of control, of equality, of opportunities, of community, of enhancement.

This promissory nature, of course, means that DIY biology and medicine as much as it is concerned with changing the here and now, is always also future-directed, guided by optimism - or pessimism - about the future. It is about tomorrow and the promises of tomorrow. Delgado argues that this promissory nature is not necessarily oriented towards the "realization of grand narratives and promises" of technological futures, but that it more often makes "mundane futures in every-day practices," "makes novelty by hacking trivial things" (71). Some scholars criticize that the DIY biology movement has been "over-hyped" (Ledford 650), that especially the media attention has "overstated and mythologized" what is going on in the movement, which is far from ground-breaking research and innovation but rather "political, artistic and educational experimentation" (Delfanti, Biohackers 115). It is not necessarily a future of sweeping transformation and upheaval - the redefinition of humanity, the realization of transhumanist dreams of longevity, immortality and extension - but a more grounded vision. DIY biology's promise is a political claim, the "promise of a future in which citizens are more autonomous as they are entitled to do biology" (Delgado 71). The practices of biohacking, Delfanti writes, are not just "hands-on approaches to technology" but "a means of creating new politics," "geared towards the development of concepts that lie at the very core of our societies, such as openness, property, freedom and autonomy" (Biohackers, 139). DIY biology can shine a light on alternative practices, alternative solutions, alternative ideologies. It creates a "public shaped by the promises and imaginaries brought about by narratives of democratization" (Meyer, "Fabric" 873).

In its essence, DIY promises a certain type of utopia. It is not just the utopia of a perfect body - Chrysanthou's "somatopia" with its material effects - but also a sociopolitical utopia. "Since Thomas More and the utopian genre's inception, utopia has been both the description of an impossible place and the impossible social relations within that place," writes Sean Grattan (4). But Grattan himself takes utopian discourse and narratives to mean more than these traditional definitions: For him, utopia is about hope for the future - "striving for another world, a better life, or at least the very pos- 
sibility of a better life" (20) - as much as a struggle between the present and its utopian potential (3). Imagining and articulating the possibility of a different world, of different social relations, of hope for the future, if we follow Grattan's train of thought, already attempts to bring that world into being. Similarly, both the ideology of DIY and its (small-scale) implementations attempt to fulfill DIY's utopian potential. It is here that the power of its promises might unfold. Affective structures, promises, desires, or hope for the future shape how we engage with biotechnologies and our own biological materiality, collectively and individually. However, as is the case with all these 'non-places' also DIY biology and medicine might fall short of its utopian promise, at least partially. Coercions and requirements to take part, questions of data privacy and security, potentials for discrimination and abuse of technologies already point into that direction.

\section{Now and Tomorrow}

As this book demonstrated, multiple pathways in our contemporary cultural moment have intersected and combined to form a new outlook on life and how it should be lived, new obligations and responsibilities, new forms of community and modes of individuation, new means of participation and agency - in short, a new "politics of hacking life" (Meyer, "Hacking" n.p.). Of course, since the inception of the first DIY biology groups and the rise in media and academic attention the configurations that led to DIY's growth have continued to evolve. It is thus worthwhile to, in the end, take a last look at the current situation. It seems like today more mundane hacks receive the most media attention, 'biohacking' and 'DIY biology' have become more common, and are covered also by mainstream magazines and media, especially when they aim at self-enhancement. Nonetheless, not surprisingly some of the more extreme forms of biohacking have gotten (critical) media attention as well, such as Josiah Zayner's public DIY CRISPR hack or his fecal transplants. Among those more extreme forms we could count 'young blood transfusion,' which is becoming popular in Silicon Valley, at least if we believe the media attention this technique hast gotten. On the practical side, more extreme practices, such as grinding, implants or magnets, have not (yet?) seen the rise that was predicted also by the widespread media coverage they received in the early 2010 s. For now, the future seems to lie in wearable technologies and, more importantly, the consumer market. As CRISPR technology - the new buzzword in the DIY scene - becomes easier and more consumer-friendly, this focus might also morph from the technological to the molecular.

Apart from the future configurations of DIY biology and medicine, this book of course could only touch upon some larger concerns in passing. Journalist Adi Robertson, lamenting that she "hacked [her] body for a future that never came," details how some biohackers see more potential for the future of biohacking in European countries. One of her sources, Amal Graafstra, founder of Dangerous Things, has witnessed a decline in sales in the US after the election of President Donald Trump in $2016 .^{3}$ While

Graafstra muses that President Trump's election resulted in a decline in optimism and excitement for the future in his target group. They now had more pressing issues to worry about than the optimization and enhancement of their bodies, cf. Robertson. 
they have recovered slightly, Graafstra is now "pessimistic about the future of biohacking in the US:" "We're the old man on the porch, and the young kids across the pond are doing the cool stuff now,' says Graafstra" (Robertson). Despite their origin in the US, Graafstra proposes that more exciting advancements and institutionalized uses of biohacking technologies will come from Scandinavian and Western European countries. A more detailed global perspective on DIY biology, thus, is worth more consideration: In different countries and regions of the world different types of biohacking are popular, often based on cultural differences and acute and actual needs (cf. Kera; Seyfried et al.). Juxtaposing the differences between DIY practices in different regions of the world, their different values, norms, connections with industry and legislation, their practitioners and their boundaries, and last but not least its effect on bodies and embodiment would deepen our understanding of the global currents, interconnections and possible downsides. We need to understand DIY biology as a transnational phenomenon that participates in trades and trade-offs around the globe, that spans more than one territory, region or nation state, that is interconnected and intersected. Some of these intersections should be examined very carefully because they risk turning from modes of participation and equality into modes of colonization and imperialism, for example when it comes to differential distributions of resources, global flows of data and use of human research subjects. ${ }^{4}$ At the same time, we need to continuously question our own understanding of research and knowledge and follow the lead of local, indigenous communities and makers, their thought systems and epistemologies.

In addition, the legal frame of DIY biology and medicine poses new questions about its regulation and legislation, patents and patent regulation, restrictions on access and knowledge: How do and should we regulate (dangerous) knowledge in the age of information? How do we keep individuals and communities safe without impinging on values such as freedom of speech and autonomy? How do we balance data sharing or data philanthropy with data security and data privacy (cf. Ajana)? Similar questions arise when we look at its moral and ethical implications: Should we allow (unending and unregulated) human enhancement? What is the worth of human life? How do we avoid slippery slopes towards new eugenics? How do we ensure accountability, lower the danger of misinformation and prevent the mis-use of technologies? Of course, such overarching questions cannot be answered in the limited scope of this book. Likewise, also the feasibility and practical application of some techniques and technologies are not part of this discussion - and not part of my area of competence. It is, however, certainly worth its time to investigate whether some of these technologies are applicable on a broader scale or feasible at all.

Nevertheless, we need to acknowledge that DIY biology and medicine is still in the process of formation, or rather, it will always remain in the process of formation: Its boundaries are still liquid - and open to debate - its configurations shifting. Morgan

For a critical examination of global data flows, cf. Keune. The clinical studies and problematic global distribution of the Covid-19 vaccines are just the latest example of differential distributions of resources and ethical dilemmas in human subject research, cf. Liu et al.; Pfeiffer and Washington. For a historical look on racial injustices in health research and the American health care system, see also Washington. 
Meyer contends that it is not yet an established "amateur science" but a "promised amateur science, a citizen science "in the making" (Domesticating 8). Its reliance on technologies and technological change means that it will probably remain "in the making." As new technologies emerge, as old technologies are re-shuffled or become more affordable, as new knowledge is produced and old knowledge solidified, the techniques used to peer into the human body, to hack biological materials, to tinker with technologies will shift as well. Some of the tools and techniques in my discussion will become obsolete, others will become routinized and expected. And because they rely so much on age-old human desires (to be involved, to become better, to transcend what we currently are) and curiosity (to know more, to know the self) we can be certain that new ones will emerge. Most of them, I would argue, will fall into either one of the categories I delineated above - or multiple ones at the same time. And since these techniques and practices stem from similar values and basic prerequisites, the questions, issues and concerns discussed here will most likely still be relevant for new, constantly evolving technologies as well. 



\section{Works Cited}

“\#privacyplease." Designated Survivor, 3.03, Netflix, 7 June 2019.

“\#scaredsh"tless." Designated Survivor, 3.08, Netflix, 7 June 2019.

23andMe. Customer Stories. www.23andme.com/stories/. Accessed 21 Aug. 2018.

-. DNA Genetic Testing \& Analysis. www.23andme.com/. Accessed 26 July 2018.

-. Research. www.23andme.com/research/. Accessed 21 Aug. 2019.

-. Introducing a Do-It-Yourself Revolution in Disease Research. 23andMe Blog, 1 Jan. 2009, blog.23andme.com/23andme-research/introducing-a-do-it-yourself-revolutionin-disease-research/. Accessed 21 Aug. 2019.

-. A Note on 23andMe's New Collaboration with GSK. 23andMe Blog, 1 Jan. 2018, blog.23andme.com/news/collaboration-with-gsk/. Accessed 21 Apr. 2021.

Abend, Pablo, et al., editors. Quantified Selves and Statistical Bodies. Spec. issue of Digital Culture \& Society vol. 2, no. 1, transcript, 2016.

Abend, Pablo, and Mathias Fuchs. "Introduction: Quantified Selves and Statistical Bodies." Quantified Selves and Statistical Bodies, edited by Pablo Abend et al., transcript, 2016.

Adams, Steve, and Sean Horlor. SHIFT: Biohacking Documentary. uploaded by Nootka Street Film Company, 21 May 2015, YouTube. Accessed 16 Apr. 2021. www.youtube.c om/watch?v=B75zyFDVPGc.

Aguiton, Sara, and Sara Tocchetti. "Is an FBI Agent a DIY Biologist Like Any Other? A Cultural Analysis of a Biosecurity Risk." Science, Technology \& Human Values, vol. 40, no. 5, 2015, pp. 825-853.

Ajana, Btihaj. "Digital Health and the Biopolitics of the Quantified Self." Digital Health, vol. 3, 2017, pp. 1-18. doi:10.1177/2055207616689509

Altman, Lawrence K. Who Goes First? The Story of Self-Experimentation in Medicine. University of California Press, 1998.

Andelfinger, Volker P. "EHealth: Grundlagen Und Bedeutung Für Die Gesundheitssysteme Heute Und Morgen.” Andelfinger and Hänisch, EHealth, pp. 25-29.

Andelfinger, Volker P., and Till Hänisch, editors. EHealth: Wie Smartphones, Apps Und Wearables Die Gesundheitsversorgung Verändern Werden. Gabler, 2016.

—. "Einleitung." Andelfinger and Hänisch, EHealth, pp. 1-4. 
Anderson, Ben. "Affective Atmospheres." Emotion, Space and Society, vol. 2, no. 2, 2009, pp. 77-81. doi:10.1016/j.emospa.2009.08.005.

-. "Affect and Biopower: Towards a Politics of Life." Transactions of the Institute of British Geographers, vol. 37, no. 1, 2012, pp. 28-43. doi:10.1111/j.1475-5661.2011.00441.x.

-. Encountering Affect: Capacities, Apparatuses, Conditions. Routledge, 2014.

Anderson, Benedict. Imagined Communities: Reflections on the Origin and Spread of Nationalism. Rev. and extended ed., 13. impression. Verso, 2003.

Anderson, Chris. Makers: The New Industrial Revolution. Crown Business, 2012.

"Arkangel." Black Mirror, 4.02., Netflix, 29 Dec. 2017.

Asprey, Dave. "What Is Biohacking? Bulletproof's Biohacking Infographic." Bulletproof Blog, blog.bulletproof.com/biohacking-infographic/. Accessed 18 June 2018.

-. "Going Bulletproof for Beginners: A Guide to Becoming Bulletproof." Bulletproof Blog, 1 June 2015, blog.bulletproof.com/bulletproof-for-beginners/. Accessed 18 June 2018.

-. Super Human: The Bulletproof Plan to Age Backwards and Maybe Even Live for Ever. Thorsons, 2019.

Atkinson, Paul. "Introduction: Do It Yourself: Democracy and Design." Journal of Design History, vol. 19, no. 1, 2006, pp. 1-10.

Attfield, Judy. Wild Things: The Material Culture of Everyday Life. Berg, 2000.

Auster, Paul. City of Glass. Reclam, 2013.

Bal, Alexandra, et al. "Mélange of Making: Bringing Children's Informal Learning Cultures to the Classroom." Boler and Ratto, DIY Citizenship: Critical, 157-169.

Balding, David, et al. Sense About Genetic Ancestry Testing. Sense About Science, 2013, archive.senseaboutscience.org/data/files/resources/119/Sense-About-Genetic -Ancestry-Testing.pdf

Banes, W. F., and Doane Hoag. American Maker. presented by Chevrolet, I Jan. 1960, archive.org/details/0548_American_Maker

Baumgaertner, Emily. As D.I.Y. Gene Editing Gains Popularity, 'Someone Is Going to Get Hurt'. 7 July 2019, www.nytimes.com/2018/05/14/science/biohackers-gene-editing-virus.h tml?action=click\&module=RelatedLinks\&pgtype=Article. Accessed 7 July 2019.

“BDNF." Lexikon Der Neurowissenschaft, Spektrumverlag. 4 Dec. 2014, www.spektrum.de /lexikon/neurowissenschaft/bdnf/1326. Accessed 10 Feb. 2020.

Bean, Jonathan, and Daniela Rosner. "Making: Movement or Brand?" interactions, vol. 21, no. 1, 2014, pp. 26-27. doi:10.1145/2541669.

Beck, Stefan. "Medicalizing Culture(S) or Culturalizing Medicine(S)." Burri and Dumit, Biomedicine as Culture, pp. 17-33.

Beck, Ulrich. Risk Society: Towards a New Modernity. Sage, 1992. Theory, Culture \& Society. Belluz, Julia. "The Bulletproof Diet Is Everything Wrong with Eating in America." Vox, 19 Dec. 2014, www.vox.com/2014/12/19/7416939/bulletproof-coffee. Accessed 21 Aug. 2018.

Benjamin, Ruha. People's Science: Bodies and Rights on the Stem Cell Frontier. Stanford University Press, 2013.

Bennett, Gaymon, et al. "From Synthetic Biology to Biohacking: Are We Prepared?" Nature biotechnology, vol. 27, no. 12, 2009, pp. 1109-11. doi:10.1038/nbt1209-1109.

Bento Bioworks. Bento Lab: Eperience Genetics Anywhere. 27 July 2018, www.bento.bio/. Accessed 27 July 2018. 
Bercovitch, Sacvan. The Puritan Origins of the American Self. 4. print. Yale University Press, 1979.

Berkhout, Suze G., and Lisa Richardson. "Identity, Politics, and the Pandemic: Why Is COVID-19 a Disaster for Feminism(S)?" History and Philosophy of the Life Sciences, vol. 42, no. 4, 2020, p. 49. doi:10.1007/s40656-020-00346-7.

Berlucchi, G., and H. A. Buchtel. "Neuronal Plasticity: Historical Roots and Evolution of Meaning." Experimental brain research, vol. 192, no. 3, 2009, pp. 307-19. doi:10.100 7/s00221-008-1611-6.

Berman, Marshall. All That Is Solid Melts into Air: The Experience of Modernity. Viking Penguin, 1988.

Berry, Jennifer. Nootropics: Types, Safety, and Risks of Smart Drugs. 18 Sep. 2019, www.med icalnewstoday.com/articles/326379.php\#over-the-counter. Accessed 10 Feb. 2020.

Biba, Erin. "Genome at Home: Biohackers Build Their Own Labs." Wired, 19 Aug. 2011, www.wired.com/2011/08/mf_diylab/. Accessed 10 Aug. 2018.

Bietz, Matthew J, and Gillian R. Hayes. "Creating Meaning in a World of Quantified Selves." Institute of Electrical and Electronics Engineers: IEEE pervasive computing: Mobile and Ubiquitous Systems, vol. 15, no. 2, 2016, pp. 82-85.

biohack.me Wiki. Who We Are. wiki.biohack.me/Who_We_Are. Accessed 28 Sep. 2019.

Boler, Megan, and Matt Ratto, editors. DIY Citizenship: Critical Making and Social Media. MIT Press, 2014.

Boltanski, L., and E. Chiapello. The New Spirit of Capitalism. Verso, 2005.

Boyd, Kenneth. "Beyond Politics: Additional Factors Underlying Skepticism of a COVID19 Vaccine." History and Philosophy of the Life Sciences, vol. 43, no. 1, 2021, 12. doi:10.10 07/s40656-021-00369-8.

Bradley, Mike. "Diabetes Patients Are Hacking Their Way Toward a Bionic Pancreas." Wired, 1 Jan. 2014, www.wired.com/2014/12/diabetes-patients-hacking-together-di y-bionic-pancreases/. Accessed 10 Aug. 2018.

Brady, Malcolm. "Covid-19 and the Power of Rules." History and Philosophy of the Life Sciences, vol. 43, no. 1, 2021, p. 11. doi:10.1007/s40656-021-00368-9.

Braun, B. "Biopolitics and the Molecularization of Life." Cultural Geographies, vol. 14, no. 1, 2007, pp. 6-28. https://journals.sagepub.com/doi/10.1177/1474474007072817.

Brodwin, Paul, editor. Biotechnology and Culture: Bodies, Anxieties, Ethics. Indiana University Press, 2000.

-. Introduction. Brodwin, Biotechnology and Culture, pp. 1-23.

Brown, Phil, et al. "Embodied Health Movements: New Approaches to Social Movements in Health." Conrad, Sociology, pp. 592-604.

Brüninghaus, Anne, and Nils Heyen. "Wissenstransfer Von Der Gesellschaft in Die Wissenschaft? Formen Und Potenziale Nicht-Zertifizierter Expertise Für Lebenswissenschaften Und Medizin." Technikfolgenabschätzung, vol. 23, no. 2, 2014, pp. 63-66.

Bulletproof Executive. Bulletproof | Power Mind and Body. www.bulletproof.com/. Accessed 21 Aug. 2018.

Bureaud, Annick, et al., editors. META-LIFE: Biotechnologies, Synthetic Biology, a-Life and the Arts. Leonardo - MIT Press e-book, 2014.

Burger, Neil, director. Limitless. Leslie Dixon, Scott Kroopf, and Ryan Kavanaugh. 2011, Film. 
Burri, Regula Valérie, and Joseph Dumit, editors. Biomedicine as Culture: Instrumental Practices, Technoscientific Knowledge, and New Modes of Life. Routledge, 2007.

-. Introduction. Burri and Dumit, Biomedicine as Culture, pp. 1-14.

Butler, Judith. Gender Trouble: Feminism and the Subversion of Identity. Routledge, 1990. Thinking Gender.

-. Precarious Life: The Powers of Mourning and Violence. Verso, 2003.

Butler, Judith, et al., editors. Vulnerability in Resistance. Duke University Press, 2016.

Butler, Mark. "Das Spiel Mit Sich. Populäre Techniken Des Selbst.” Express Yourself! Europas Kulturelle Kreativität Zwischen Markt Und Underground, edited by Eva v. Kimminich et al., transcript-Verlag, 2007, pp. 75-101.

—. Das Spiel Mit Sich: (Kink, Drugs \& Hip-Hop). Kulturverl. Kadmos, 2014. Kaleidogramme Bd. 103.

Cammack, Richard, and Teresa K. Attwood, editors. Oxford Dictionary of Biochemistry and Molecular Biology. Rev. ed., 2. ed. Oxford Univ. Press, 2006.

Campbell, Colin. "The Craft Consumer: Culture, Craft and Consumption in a Postmodern Society." Journal of Consumer Culture, vol. 5, no. 1, 2005, pp. 23-42. doi:10.1177/146 9540505049843.

Cariaso, Michael, and Greg Lennon. "SNPedia: A Wiki Supporting Personal Genome Annotation, Interpretation and Analysis." Nucleic Acids Research, vol. 40, Database issue, 2012, D1308-12. doi:10.1093/nar/gkr798.

Catherine Reitman, creator. "Consent." Workin' Moms, 2.05, CBC Television/Netflix, 30 Jan. 2018.

Chadarevian, Soraya de, and Roberta Raffaetà. "COVID-19: Rethinking the Nature of Viruses." History and Philosophy of the Life Sciences, vol. 43, no. 1, 2021, p. 2. doi:10.100 7/s40656-020-00361-8.

Chai Biotechnologies. OpenPCR: The \$499 Open Source PCR Machine / Thermal Cycler. 30 July 2018, openpcr.org/. Accessed 10 Aug. 2018.

-. OpenPCR About. 27 Aug. 2018, openpcr.org/about/. Accessed 5 Oct. 2019.

Chatterjee, Arjan. "Cosmetic Neurology: The Controversy over Enhancing Movement, Mentation, and Mood." Neurology, vol. 63, no. 6, 2004, pp. 968-974. doi:10.1212/01.WNL.0000138438.88589.7C.

Chrysanthou, Marc. "Transparency and Selfhood: Utopia and the Informed Body." Social Science \& Medicine, vol. 54, 2002, pp. 469-479.

Clark, Andy. "Re-Inventing Ourselves: The Plasticity of Embodiment, Sensing, and Mind." The Journal of Medicine and Philosophy, vol. 32, no. 3, 2007, pp. 263-282. doi: $10.1080 / 03605310701397024$.

Clough, Patricia T. "The Affective Turn: Political Economy, Biomedia, and Bodies.” Gregg and Seigworth, The Affect Theory, pp. 206-225.

Coker, Cheo Hodari, creator. Marvel's Luke Cage. Netflix, 2016-2018.

Collier, Stephen J., and Andrew Lakoff. "On Regimes of Living." Ong and Collier, Global Assemblages: Technology, pp. 22-39.

Condliffe, Jamie. "A DIY EpiPen Costs \$30 to Make." MIT Technology Review, 20 Sep. 2016, www.technologyreview.com/s/602422/it-costs-30-to-make-a-diy-epipe n-and-heres-the-proof/. Accessed 24 July 2018. 
Conrad, Peter. The Medicalization of Society: On the Transformation of Human Conditions into Treatable Disorders. Johns Hopkins University Press, 2007.

-, editor. The Sociology of Health $\&$ Illness: Critical Perspectives. $8^{\text {th }}$ ed. Worth Publishers, 2009.

Coole, Diana H., and Samantha Frost. New Materialisms: Ontology, Agency, and Politics. Duke University Press, 2010.

Coupette, Jan. "Digitale Disruption Erfordert Bewegung - Das Internet of Everything." Wirtschaftsinformatik \& Management, no. 2, 2014, pp. 20-29.

Coward, Cameron. "CyberPunk Yourself - Body Modification, Augmentation, and Grinders.” Hackaday, 12 Oct. 2015, hackaday.com/2015/10/12/cyberpunk-yourself-b ody-modification-augmentation-and-grinders/. Accessed 7 Aug. 2018.

Cowie, Jefferson, and Nick Salvatore. "The Long Exception: Rethinking the Place of the New Deal in American History." International Labor and Working-Class History, vol. 74, 2008, pp. 1-32. https://ecommons.cornell.edu/handle/1813/75045

Croly, Herbert David. The Promise of American Life. 1909.

Csordas, Thomas J. "Computerized Cadavers. Shades of Being and Representation in Virtual Reality." Brodwin, Biotechnology and Culture, pp. 173-192.

D’Adesky, Anne-Christine. "Was the EpiPen Hack Ethical?” KQED, 5 June 2017, www.kq ed.org/futureofyou/323092/was-the-epipen-hack-ethical. Accessed 24 July 2018.

Dana Lewis \& the \#OpenAPS community. OpenAPS.Org - \#WeAreNotWaiting to Reduce the Burden of Type 1 Diabetes. openaps.org/. Accessed 10 Aug. 2018.

-. OpenAPS Outcomes. 23 Apr. 2021, openaps.org/outcomes/. Accessed 23 Apr. 2021.

Davis, Lennard J., and David B. Morris. "Biocultures Manifesto." New Literary History, vol. 38, no. 3, 2007, pp. 411-418. doi:10.1353/nlh.2007.0046.

Dean, Mitchell. Governmentality: Power and Rule in Modern Society. Sage, 1999.

Deleuze, Gilles. Essays Critical and Clinical,. trans. D. W. Smith \& M. A. Greco. Univ Of Minnesota Press, 1997.

Delfanti, Alessandro. "Users and Peers: From Citizen Science to P2P Science." Journal of Science Communication, vol. 9, no. 1, 2010, pp. 1-5.

-. Biohackers: The Politics of Open Science. Pluto Press, 2013.

—. "Is Do-It-Yourself Biology Being Co-Opted by Institutions?" Bureaud et al., METALIFE: Biotechnologies, Synthetic, n.p.

Delgado, Ana. "DIYbio: Making Things and Making Futures." Futures, vol. 48, 2013, pp. 65-73. doi:10.1016/j.futures.2013.02.004.

Delgado, Ana, and Blanca Callen. "Do-It-Yourself Biology and Electronic Waste Hacking: A Politics of Demonstration in Precarious Times." Public Understanding of Science, vol. 26, no. 2, 2017, pp. 179-194. doi:10.1177/0963662516647348.

Dewey, J. The Public and Its Problems: An Essay in Political Inquiry. Penn State University Press, 1927.

Diagnosis. Netflix, 16 Aug. 2019.

Diana, Carla. "How I Learned to Stop Worrying and Love the Hackers." Interactions, vol. 15, no. 2, 2008, p. 46. doi:10.1145/1340961.1340973.

Dickenson, Donna. Me Medicine Vs. We Medicine: Reclaiming Biotechnology for the Common Good. Columbia University Press, 2013.

Ditter, Christian, creator. Biohackers. Netflix, 20 Aug. 2020. 
DIYgenomics. diygenomics.org/. Accessed 27 July 2018.

DNAFit Life Sciences Limited/Prenetics. DNAFit-Genetic Information for Fitness $\&$ Nutrition (US). 1 Jan. 2018, www.dnafit.com/us/. Accessed 6 Sep. 2018.

Dolby, Sandra K. Self-Help Books: Why Americans Keep Reading Them. University of Illinois Press, 2005.

Dolgin, Elie. "Personalized Investigation." Nature Medicine, vol. 16, no. 9, 2010, pp. 953955. doi:10.1038/nmo910-953.

Donovan, Matthew C. A Case Study of Organizational, Pharmaceutical, and Biohacker Narratives. Dissertation, Arizona State University, 2019, search.proquest.com/openview/ 1d3c312f919a3c7ab4c845973926b7e7/1?pq-origsite $=$ gscholar\&cbl=18750\&diss=y.

Druckermann, Kevin, et al., creator. (Un)Well. Netflix, 12 Aug. 2020.

Ducharme, Jamie. "A Major Drug Company Now Has Access to 23andMe's Genetic Data. Should You Be Concerned?" Time, 26 July 2018, time.com/5349896/23andme-glaxo-s mith-kline/. Accessed 23 Oct. 2020.

Duden, Barbara, and Silja Samerski. "Pop Genes": An Investigation of "The Gene" in Popular Parlance." Burri and Dumit, Biomedicine as Culture, pp. 167-189.

Dumit, Joseph. Drugs for Life: How Pharmaceutical Companies Define Our Health. Duke University Press, 2012.

Dumit, Joseph, and Regula Valérie Burri. "Epilogue: Indeterminate Lives, Demands, Relations: Emergent Bioscapes." Burri and Dumit, Biomedicine as Culture, pp. 223228.

Dunbar-Hester, Christina. "Radical Inclusion? Locating Accountability in Technical DIY." Boler and Ratto, DIY Citizenship: Critical, pp. 75-88.

Dynamic DNA Laboratories. Discover Yourself: DNA Testing | Dynamic DNA Labs. 25 July 2018, dynamicdnalabs.com/. Accessed 26 July 2018.

Eggers, Dave. The Circle: A Novel. Vintage Books, a division of Random House LLC, 2013.

Eisenberg, D. M., et al. "Trends in Alternative Medicine Use in the United States, 19901997: Results of a Follow-up National Survey." JAMA, vol. 280, no. 18, 1998, pp. 15691575. doi:10.1001/jama.280.18.1569.

Elias, Norbert. The Civilizing Process. Urizen Books, 1978-1982.

Elliott, Anthony. Making the Cut: How Cosmetic Surgery Is Transforming Our Lives. Reaktion Books, 2008.

Elliott, Carl. Better Than Well: American Medicine Meets the American Dream. W.W. Norton, 2004.

Emerson, Ralph Waldo. "Nature 1836." Emerson and Ziff, Nature and Selected, pp. 35-82.

—. "Self-Reliance." Emerson and Ziff, Nature and Selected, pp. 175-204.

Emerson, Ralph Waldo, and Larzer Ziff, editors. Nature and Selected Essays. Penguin, 2003. Penguin Classics.

Erickson, Mark. Science, Culture and Society: Understanding Science in the $21^{\text {st }}$ Century. Polity Press, $20162^{\text {nd }}$ edition.

Federal Trade CommissionFTC and FDA Send Warning Letters to Companies Selling Dietary Supplements Claiming to Treat Alzheimer's Disease and Remediate or Cure Other Serious Illnesses Such as Parkinson's, Heart Disease, and Cancer, 11 Feb. 2019. Accessed 28 Aug. 2019.

Field, Dawn. Biocode: The New Age of Genomics. Oxford Univ Press, 2016.

Fitbit, Inc. Why Fitbit. www.fitbit.com/whyfitbit. Accessed 27 Sep. 2019. 
-. Fitbit Official Site for Activity Trackers \& More. 11 Sep. 2018, www.fitbit.com/home. Accessed 12 Sep. 2018.

Fleck, Ludwik. Entstehung Und Entwicklung Einer Wissenschaftlichen Tatsache; Einführung in Die Lehre Vom Denkstil Und Denkkollektiv. Suhrkamp, 1935.

Flichy, Patrice. "Lindividualisme Connecté Entre La Technique Numérique Et La Société." Réseaux, 2004, pp. 17-51.

Fluck, Winfried. Das Kulturelle Imaginäre: Eine Funktionsgeschichte Des Amerikanischen Romans 1790-1900. Suhrkamp, 1997.

Foucault, Michel. The History of Sexuality: Volume I: An Introduction. transl. by Robert Hurley. Random House, Inc., 1980.

-. "About the Beginning of the Hermeneutics of the Self: Two Lectures at Dartmouth." Political Theory, vol. 21, no. 2, 1993, pp. 198-227.

Four Thieves Vinegar Collective. Four Thieves Vinegar: Home. fourthievesvinegar.org/. Accessed 24 July 2018.

-. Our Mission. fourthievesvinegar.org/our-mission. Accessed 29 Aug. 2019.

—. Epi Pencil. 1 Jan. 2016, archive.org. archive.org/details/EpiPencil_201801. Accessed 24 July 2018.

-. "Introducing the EpiPencil." Four Thieves Vinegar Collective, 19 Sep. 2016, fourthievesv inegar.org/?b=001. Accessed 19 Apr. 2021.

—. Epi Pencil Update. 7 Oct. 2017, archive.org. archive.org/details/EpiPencilUpdate. Accessed 24 July 2018.

Fox, Maggie. "Memory Pills Are a Hoax, FTC Said in Suit Against Company." NBCNews, 9 Jan. 2017, www.nbcnews.com/health/health-news/jellyfish-memory-supplement -prevagen-hoax-ftc-says-n704886.

Fox, Nick J. "Personal Health Technologies, Micropolitics and Resistance: A New Materialist Analysis." Health, vol. 21, no. 2, 2017, pp. 136-153. doi:10.1177/1363459315590248.

Frank, Arthur W. The Wounded Storyteller: Body, Illness, and Ethics. 2. ed. Univ. of Chicago Press, 2013.

Franklin, Benjamin. Poor Richard's Almanack: Being the Choicest Morsels of Wisdom, Written During the Years of the Almanack's Publication, by That Well-Known Savant, Dr. Benjamin Franklin. Peter Pauper Press, 1994 (1732-1758).

Fraser, Nancy. "Rethinking the Public Sphere: A Contribution to the Critique of Actually Existing Democracy." Social Text, 25/26, 1990, pp. 56-80. doi:10.2307/466240.

Frauenfelder, Mark. Made by Hand: Searching for Meaning in a Throwaway World. Portfolio / Penguin, 2010.

Friedman, Sharon M., editor. Communicating Uncertainty: Media Coverage of New and Controversial Science. Erlbaum Associates, 1999. LEA's Communication Series.

Frost, Samantha. Biocultural Creatures: Toward a New Theory of the Human. Duke University Press, 2016.

Fukuyama, Francis. "Transhumanism: The World's Most Dangerous Ideas." Foreign Policy, no. 144, 2004, pp. 42-43. doi:10.2307/4152980.

Gauntlett, David. Making Is Connecting: The Social Meaning of Creativity, from DIY and Knitting to YouTube and Web 2.0. Polity Press, 2011. 
Gelber, Steven M. Hobbies: Leisure and the Culture of Work in America. Columbia University Press, 1999.

Genova, Lisa. Still Alice. iUniverse/Pocket Books, 2007.

Gibson, William. Neuromancer. Ace Books, 1984.

Giddens, Anthony. The Consequences of Modernity. Stanford UP, 1990.

-. Modernity and Self-Identity. Polity Press, 1991.

Glatzer, Richard, and Wash Westmoreland, directors. Still Alice. 2015.

Goldstein, Carolyn M. Do It Yourself: Home Improvement in $20^{\text {th }}$-Century America. National Building Museum; Princeton Architectural Press, 1998.

Grattan, Sean Austin. Hope Isn't Stupid. University of Iowa Press, 2017.

Greenbaum, Dov. “Biology's Brave New World.” Science, vol. 369, no. 6508, 2020, p. 1170. doi:10.1126/science.abe1950.

Greene, Jeremy A. "Do-It-Yourself Medical Devices. Technology and Empowerment in American Health Care." The New England Journal of Medicine, vol. 374, no. 4, 2016, pp. 305-308.

Greenfield, Ben. Boundless: Upgrade Your Brain, Optimize Your Body \& Defy Aging. Victory Belt Publishing Inc, 2020.

Gregg, Melissa, and Gregory J. Seigworth, editors. The Affect Theory Reader. Duke University Press, 2010.

Grewe-Salfeld, Mirjam. A Dialectic of (in)Visibility: The Cultural Imaginary of Multiple Sclerosis. Unpublished Master Thesis, Universität Potsdam, 2016.

Grossberg, Lawrence. Cultural Studies in the Future Tense. Duke University Press, 2010.

Grossberg, Lawrence / interviewed by Seigworth and Gregg. "Affect's Future: Rediscovering the Virtual in the Actual." Gregg and Seigworth, The Affect Theory, pp. 309-338.

Gruber, Karl. "Biohackers: A Growing Number of Amateurs Join the Do-It-Yourself Molecular Biology Movement Outside Academic Laboratories." EMBO reports, vol. 20, no. 6, 2019, pp. 1-3. doi:10.15252/embr.201948397.

Habermas, Jürgen. The Structural Transformation of the Public Sphere: An Inquiry into a Category of Bourgeois Society. MIT Press, 1989.

Hall, Lena E. A Psychology of American Culture. Edwin Mellen Press, 2015.

Hamblin, James. "7 Biohacks to Master Before Worrying About Other Biohacks." The Atlantic, www.theatlantic.com/health/archive/2019/03/top-biohacks/584 584/. Accessed 5 July 2019.

Hamburg, Margaret A., and Francis S. Collins. "The Path to Personalized Medicine." The New England journal of medicine, vol. 363, no. 4, 2010, pp. 301-304. doi:10.1056/NEJM p1006304.

Hänisch, Till. "EHealth - Eine Begriffbestimmung." Andelfinger and Hänisch, EHealth, pp. 5-10.

Happe, Kelly E., et al., editors. Biocitizenship: The Politics of Bodies, Governance and Power. New York University Press, 2018.

Harari, Yuval Noah. Homo Deus: A Brief History of Tomorrow. Vintage, 2017.

Haraway, Donna Jeanne. "Situated Knowledges. The Science Question in Feminism and the Privilege Partial Perspective." Feminist Studies, vol. 14, no. 3, 1988, pp. 575-599. 
—. "A Cyborg Manifesto: Science, Technology, and Socialist-Feminism in the Late Twentieth Century." Manifestly Haraway, edited by Donna Jeanne Haraway, Univ Of Minnesota Press, 2016, pp. 3-90. Posthumanities 37.

Harris, Anna, et al. "Autobiologies on YouTube: Narratives of Direct-to-Consumer Genetic Testing." New genetics and society, vol. 33, no. 1, 2014, pp. 60-78. doi:10.1080/146 36778.2014.884456.

Hartley, John. Uses of Television. Routledge, 1999.

Harvard Medical School/Wyss Institute. The Harvard Personal Genome Project (PGP) - Enabling Participant-Driven Science. pgp.med.harvard.edu/. Accessed 27 July 2018.

Hatch, Mark. The Maker Movement Manifesto: Rules for Innovation in the New World of Crafters, Hackers, and Tinkerers. McGraw-Hill Education, 2014.

Have, Henk A.M.J. ten. "Genetics and Culture: The Geneticization Thesis." Medicine, Health Care and Philosophy, vol. 4, no. 3, 2001, pp. 295-304. doi:10.1023/A:101209081 0798.

Hawthorne, Nathaniel. "The Birthmark." Young Goodman Brown, and Other Short Stories, edited by Nathaniel Hawthorne, Dover Publications, 1992 [1846).

Hayles, Nancy Katherine. How We Became Posthuman: Virtual Bodies in Cybernetics, Literature, and Informatics. Univ. of Chicago Press, 1999.

Heinemann, Torsten. "Biological Citizenship." Encyclopedia of Global Bioethics, edited by H. ten Have, Living Reference Work, Springer International Publishing, 2016, pp. 1-7.

Hemmings, Clare. "INVOKING AFFECT." Cultural Studies, vol. 19, no. 5, 2005, pp. 548-67. doi:10.1080/09502380500365473.

Hill, Kashmir. "Taking My Measure." Forbes : business and finance, 2011, pp. 98-104.

Hins, Alice. "Magnet Implants? Welcome to the World of Medical Punk." The New York Times, 12 May 2018, www.nytimes.com/2018/05/12/us/grindfest-magnet-implantsbiohacking.html. Accessed 5 July 2019.

Hinson, Katrina, and Ben Sword. "Illness Narratives and Facebook: Living Illness Well." Humanities, vol. 8, no. 2, 2019, p. 106. doi:10.3390/h8020106.

Hodson, Hal. "Smart Health Monitors to Predict Your Medical Future." New Scientist, 5 Nov. 2014, www.newscientist.com/article/mg22429942-900-smart-health-monito rs-to-predict-your-medical-future/. Accessed 9 May 2017.

Holloway, Karla F. C. Private Bodies, Public Texts: Race, Gender, and a Cultural Bioethics. Duke University Press, 2011.

Hoover, Herbet. October 22, 1928: Principles and Ideals of the United States Government. Miller Center. 1 Jan. 2016, millercenter.org/the-presidency/presidential-speeches/october -22-1928-principles-and-ideals-united-states-government. Accessed 10 Feb. 2020.

Horowitz, Andy. Pre-Existing Conditions: Pandemics as History. Items: Insights from the Social Sciences. 9 July 2020, items.ssrc.org/covid-19-and-the-social-sciences/disast er-studies/pre-existing-conditions-pandemics-as-history/. Accessed 29 Mar. 2021.

Hoy, Matthew B. "Personal Activity Trackers and the Quantified Self." Medical reference services quarterly, vol. 35, no. 1, 2016, pp. 94-100.

Huber, Richard M. The American Idea of Success. Pushcart Press, 1987.

Huxley, Aldous. Brave New World and Brave New World Revisited. 2005/1932 [reprinted]. Harper Perennial Modern Classics. 
Jen, Clare. "Do-It-Yourself Biology, Garage Biology, and Kitchen Science: A Feminist Analysis of Bio-Making Narratives." Knowing New Biotechnologies: Social Aspects of Technological Convergence, edited by M. Wienroth and E. Rodrigues, Taylor \& Francis, 2015, 125-141.

Jewell, Tim, and Deborah Weatherspoon. "Guide to Biohacking: Types, Safety, and How to." healthline, 2 Jan. 2019, www.healthline.com/health/biohacking. Accessed 5 July 2019.

Jordan, Gemma (author). "Upgrade." Dark Net. Showtime, 28 Jan. 2016.

Kampmark. "The Pandemic Surveillance State: An Enduring Legacy of COVID-19." Journal of Global Faultlines, vol. 7, no. 1, 2020, p. 59. doi:10.13169/jglobfaul.7.1.0059.

Kelty, Christopher M. "Outlaw, Hackers, Victorian Amateurs: Diagnosing Public Participation in the Life Sciences Today." Journal of Science Communication, vol. 9, no. 1, 2010, pp. 1-8.

Kera, Denisa. "Hackerspaces and DIYbio in Asia: Connecting Science and Community with Open Data, Kits and Protocols." Journal of Peer Production, no. 2, 2012, www.digitalartarchive.at/fileadmin/user_upload/Virtualart/PDF/984_5_diybi o-in-asia-libre.pdf.

Keulartz, Jozef, and Henk van den Belt. "DIY-Bio - Economic, Epistemological and Ethical Implications and Ambivalences." Life Sciences, Society and Policy, vol. 12, no. 7, 2016, pp. 1-19. doi:10.1186/s40504-016-0039-1.

Keune, Jason David. “Considering Power Relations in Citizen Science.” The American Journal of Bioethics, vol. 19, no. 8, 2019, pp. 48-49. doi:10.1080/15265161.2019.1619863.

Khotala, Cindy. Rapid Response, Slow Adjustment. Nessling Foundation. 28 Apr. 2020, ww w.nessling.fi/from-grant-receiver/rapid-response-slow-adjustment/?lang=en. Accessed 30 Mar. 2021.

Kickstarter. OpenPCR - Open Source Biotech on Your Desktop. www.kickstarter.com/project s/930368578/openpcr-open-source-biotech-on-your-desktop/description. Accessed 10 Aug. 2018.

Klayman, Alison, director. Take Your Pills. Netflix, 2018, Netflix.

Kolata, Gina, et al. "Chinese Scientist Claims to Use Crispr to Make First Genetically Edited Babies." New York Times, 26 Nov. 2018, www.nytimes.com/2018/11/26/health/ gene-editing-babies-china.html. Accessed 9 May 2019.

Kolst $\varnothing$, Stein D. "Scientific Literacy for Citizenship: Tools for Dealing with the Science Dimension of Controversial Socioscientific Issues." Science Education, vol. 85, no. 3, 2001, pp. 291-310. doi:10.1002/sce.1011.

Kramer, Peter D. Listening to Prozac. Penguin Books, 1997/1993.

Krause, Nicole M., et al. "The Polls-Trends." Public Opinion Quarterly, 2019, doi:10.1093/ poq/nfzo41.

Krupar, Shiloh, and Nadine Ehlers. "Biocultures: A Critical Approach to Mundane Biomedical Governance." Culture, Theory and Critique, 2021, pp. 1-17. doi:10.1080/14 735784.2020.1857810.

Kunow, Rüdiger. Material Bodies: Biology and Culture in the United States. $1^{\text {st }}$ ed. Universitätsverlag Winter, 2018. American Studies - a Monograph Series v.286.

Lake, Christina Bieber. Prophets of the Posthuman: American Fiction, Biotechnology, and the Ethics of Personhood. University of Notre Dame, 2013. 
Landecker, Hannah. "Living Differently in Time: Plasticity, Temporality and Cellular Biotechnologies." Culture Machine, vol. 7, 2005, www.culturemachine.net/index.php $/ \mathrm{cm} / \mathrm{rt} /$ printerFriendly/26/33.

Latham, Alan, and Derek P. McCormack. "Thinking with Images in NonRepresentational Cities: Vignettes from Berlin." Area, vol. 41, no. 3, 2009, pp. 252-262. doi:10.1111/j.1475-4762.2008.00868.x.

Latour, Bruno, and Steve Woolgar. Laboratory Life: The Construction of Scientific Facts. with a new postscript and index by the authors. Princeton University Press, 1986 (1979).

Ledford, Heidi. “Life Hackers'." Nature, vol. 467, 2010, pp. 650-652.

Lee, Caroline. Do-It-Yourself Democracy: The Rise of the Public Engagement Industry. Oxford University Press, 2014.

Lee, James. The Biohacking Manifesto: The Scientific Blueprint for a Long, Healthy and Happy Life Using Cutting Edge Anti-Aging and Neuroscience Based Hacks. 2x Helix Publishing, 2015.

Lee, Stan, and Jack Kirby. X-Men. Marvel Comics/Marvel Entertainment, 1963, 20112020. Comic Books/Film.

Lemke, Thomas. "Disposition and Determinism - Genetic Diagnostics in Risk Society." The Sociological Review, vol. 52, no. 4, 2004, pp. 550-566. doi:10.1111/j.1467-954X.2004. 00495.x.

-. "Beyond Foucault: From Biopolitics to the Government of Life." Governmentality. Current Issues and Future Challenges, edited by Ulrich Bröckling et al., Routledge, 2010, pp. 165-184.

Levina, Marina. "Googling Your Genes: Personal Genomics and the Discourse of Citizen Bioscience in the Network Age." Journal of Science Communication, vol. 9, no. 1, 2010, pp. 1-8.

Levine, Faythe, and Cortney Heimerl. Handmade Nation: The Rise of DIY, Art, Craft, and Design. Princeton Architectural Press, 2008.

Levy, Steven. Hackers: Heroes of the Computer Revolution. $1^{\text {st }}$ ed. Anchor Press, 1984.

Lewis, Dana. "Experience: I Built My Own Pancreas." The Guardian, 20 July 2018, www.theguardian.com/lifeandstyle/2018/jul/20/diabetes-experience-i-builtmy-own-pancreas. Accessed 10 Aug. 2018.

-. "History and Perspective on DIY Closed Looping." Journal of diabetes science and technology, vol. 13, no. 4, 2019, pp. 790-793. doi:10.1177/1932296818808307.

Lewis, Tim. "Bulletproof Coffee's Dave Asprey: Why Healthy Eating and Exercise Aren't Enough." The Guardian, 14 May 2017, www.theguardian.com/lifeandstyle/2017/may/1 4/bulletproof-coffee-dave-asprey-eat-healthy-exercise-interview. Accessed 21 Aug. 2018.

Lidskog, Rolf. "Book Reviews : Ulrich Beck: The Risk Society. Towards a New Modernity. London: Sage, 1992." Acta Sociologica, vol. 36, no. 4, 1993, pp. 400-403. doi:10.1177/00 0169939303600409.

Liu, Yangzi, et al. "Multivalue Ethical Framework for Fair Global Allocation of a COVID19 Vaccine." Journal of Medical Ethics, vol. 46, no. 8, 2020, pp. 499-501. doi:10.1136/me dethics-2020-106516.

Lock, Margaret. "The Eclipse of the Gene and the Return of Divination." Current Anthropology, vol. 46, 2005, pp. 47-70. 
Lorenzini, Daniele. "Biopolitics in the Time of Coronavirus." Critical Inquiry, vol. 47, S2, 2021, pp. 40-45. doi:10.1086/711432.

Lowenberg, June S., and Fred Davis. "Beyond Medicalisation-Demedicalisation: The Case of Holistic Health." Sociology of Health \& Illness, vol. 16, no. 579, 1994, pp. 579599.

Lupton, Deborah. Medicine as Culture: Illness, Disease and the Body in Western Societies. Sage, 1994.

-. "Risk as Moral Danger: The Social and Political Function of Risk Discourse in Public Health." Conrad, Sociology, pp. 460-467.

-. "Self-Tracking Cultures: Towards a Sociology of Personal Informatics." Designing Futures. The Future of Design: Proceedings of the $26^{\text {th }}$ Australian Computer-Human Interaction Conference: $2^{\text {nd }}-5^{\text {th }}$ December 2014, University of Technology, Sydney, 2/12/2014 - 5/12/2014, Sydney, New South Wales, Australia, The $26^{\text {th }}$ Australian ComputerHuman Interaction Conference, edited by Toni Robertson. New York, NY, USA. ACM, 2014, pp. 77-86.

-. The Quantified Self: A Sociology of Self-Tracking. Polity, 2016.

Lyon, Jeff. "Significant Increases in EpiPen Price." JAMA, vol. 316, no. 14, 2016, p. 1439. d oi:10.1001/jama.2016.14178.

Mackenzie, Catriona. "Imagining Oneself Otherwise." Relational Autonomy: Feminist Perspectives on Autonomy, Agency, and the Social Self, edited by Catriona Mackenzie and Natalie Stoljar, Oxford University Press, 2000.

Mackenzie, Catriona, et al. "Introduction: What Is Vulnerability, and Why Does It Matter for Moral Theory?" Vulnerability: New Essays in Ethics and Feminist Philosophy, edited by Catriona Mackenzie et al., Oxford University Press, 2013, pp. 1-29.

Majmudar, Maulik D., et al. "The Quantified Patient of the Future: Opportunities and Challenges." Healthcare (Amsterdam, Netherlands), vol. 3, no. 3, 2015, pp. 153-156. doi:10.1016/j.hjdsi.2015.02.001.

Make Magazine, editor. Back Yard Biology. Vol. 7, 2006.

Malabou, Catherine. What Should We Do with Our Brain? $2^{\text {nd }}$ ed. Fordham University, 2008 (2004).

Mann, Steve. "Maktivism: Authentic Making for Technology in the Service of Humanity." Boler and Ratto, DIY Citizenship: Critical, pp. 29-51.

Manson, Graeme and John Fawcett, creator. Orphan Black. Space/BBC America/Netflix. 2013-2017.

Martin, Emily. "Mind-Body Problems." American Ethnologist, vol. 27, no. 3, 2000, pp. 569590. doi:10.1525/ae.2000.27.3.569.

—. "The Pharmaceutical Person." BioSocieties, vol. 1, no. 3, 2006, pp. 273-287. doi:10.1017 /S1745855206003012.

Martin, Lisa. "OpenAPS Offers Open Source Tools for Diabetes Management." Make Magazine, 31 Mar. 2017, makezine.com/2017/03/31/openaps/.

Massumi, Brian. "The Autonomy of Affect." Cultural Critique, vol. 31, 1995, pp. 83-109.

-. Parables for the Virtual: Movement, Affect, Sensation. Duke Univ. Press, 2002.

Mauss, Marcel. "Techniques of the Body." Zone 6: Incorporations, edited by Jonathan Crary and Sanford Kwinter, Zone, 1992, pp. 454-477. 
McCormack, Donna, and Suvi Salmenniemi. "The Biopolitics of Precarity and the Self." European Journal of Cultural Studies, vol. 19, no. 1, 2016, pp. 3-15. doi:10.1177/136754941 5585559.

McGee, Micki. Self-Help, Inc: Makeover Culture in American Life. Oxford University Press, 2005.

Mcinerney, Claire, et al. "The Flow of Scientific Knowledge from Lab to the Lay Public." Science Communication, vol. 26, no. 1, 2004, pp. 44-74. doi:10.1177/1075547004267024.

McKinlay, John B. "A Case for Refocusing Upstream: The Political Economy of Illness." Conrad, Sociology, pp. 578-591.

McNeil, Donald G. "Fighting Cervical Cancer with Vinegar and Ingenuity." New York Times, 26 Sep. 2011, www.nytimes.com/2011/09/27/health/27cancer.html. Accessed 22 Nov. 2018.

Meisel, Ari R. Intro to Biohacking: How to Be Smarter, Stronger, and Happier. CreateSpace Independent Publishing Platform, 2014.

Melendez, Steven. "Under My Skin: The New Frontier of Digital Implants." Fast Company, www.fastcompany.com/3059769/ive-got-you-under-my-skin-the-new-fronti er-of-digital-implants. Accessed 7 Aug. 2018.

Meyer, Morgan. "Build Your Own Lab: Do-It-Yourself Biology and the Rise of Citizen Biotech-Economies." Journal of Peer Production, no. 2, 2012.

-. Domesticating and Democratizing Science: A Geography of Do-It-Yourself Biology. Paris. Centre de Sociologie de l'Innovation, 2013. Papiers De Recherche Du CSI - CSI Working Papers Series, vol. 032, www.csi.mines-paristech.fr/working-papers/WP/ WP_CSI_032.pdf.

—. "Hacking Life? The Politics and Poetics of DIY Biology." Bureaud et al., META-LIFE: Biotechnologies, Synthetic, n.p.

-. "The Fabric of the Public in Debates About Gene Editing." Environmental Communication, vol. 14, no. 7, 2020, pp. 872-876. doi:10.1080/17524032.2020.1811477.

MoodPanda. MoodPanda: Your Supportive Mood Diary. moodpanda.com/. Accessed 10 Feb. 2020.

Moodscope. Moodscope: Lift Your Mood with a Little Help from Your Friends. www.moodsco pe.com/. Accessed 10 Feb. 2020.

Moore, Phoebe. "Tracking Bodies, the 'Quantified Self', and the Corporeal Turn." Handbook of the International Political Economy of Production, edited by Kees van der Pijl, Edward Elgar Publishing, 2015, pp. 394-408.

More, Max. "The Philosophy of Transhumanism." The Transhumanism Reader: Classical and Contemporary Essays on the Science, Technology, and Philosophy of the Human Future, edited by Max More and Natasha Vita-More, John Wiley and Sons, 2013.

Mukherjee, Siddhartha. The Gene: An Intimate History. First Scribner hardcover ed. Scribner, 2016.

Murphy, Heather. "Playing Catch a Killer with a Room Full of Sleuths." New York Times, 5 Oct. 2019, www.nytimes.com/2019/10/05/us/genetic-genealogy-guidelines-privac y.html.

Murphy, Samantha. "The Most Connected Man Is You, Just a Few Years from Now." Mashable, 21 Aug. 2014, mashable.com/2014/08/21/most-connected-man/?europe=t rue\#.TvG5VUoFGq3. Accessed 1 Aug. 2018. 
MyLAB Box ${ }^{\mathrm{TM}}$ : At Home STD Tests / Test Kits for All Common STDs. www.mylabbox.com/. Accessed 9 Sep. 2019.

Nafus, Dawn, and Jamie Sherman. "This One Does Not Go up to 11: The Quantified Self Movement as an Alternative Big Data Practice." International Journal of Communication, vol. 8, 2014, pp. 1784-1794.

National Center for Complementary and Integrative Health. Complementary, Alternative, or Integrative Health: What's in a Name? 1 Jan. 2011, nccih.nih.gov/health/integrativehealth. Accessed 10 Feb. 2020.

Neff, Gina, and Dawn Nafus. Self-Tracking. MIT Press, 2016.

Neil Squire Society/Makers Making Change. Makers Making Change. www.makersmaki ngchange.com/. Accessed 10 Aug. 2018.

Nelkin, Dorothy. Selling Science: How the Press Covers Science and Technology. Rev. ed. Freeman, 1995.

Nelkin, Dorothy, and M. Susan Lindee. The DNA Mystique: The Gene as a Cultural Icon. Freeman, 1995.

Nesse, Randolph. The Body Is Not a Machine. Arizona State University Center for Evolution and Medicine. 4 Feb. 2016, evmed.asu.edu/blog/body-not-machine. Accessed 10 May 2019.

Nordgren, A., and E. T. Juengst. "Can Genomics Tell Me Who I Am? Essentialistic Rhetoric in Direct-to-Consumer DNA Testing." New Genetics and Society, vol. 28, no. 2, 2009, pp. 157-172. doi:10.1080/14636770902901595.

Nussbaum, Martha Craven. Frontiers of Justice: Disability, Nationality, Species Membership. The Belknap Press of Harvard University Press, 2007.

Oberhaus, Daniel. "Meet the Anarchists Making Their Own Medicine." Motherboard, 26 July 2018, motherboard.vice.com/en_us/article/43pngb/how-to-make-your-own-m edicine-four-thieves-vinegar-collective. Accessed 28 Aug. 2018.

O'Connell, Mark. To Be a Machine: Adventures Among Cyborgs, Utopians, Hackers, and the Futurists Solving the Modest Problem of Death. Granta Books, 2018.

O'Neil, Paul. The End and the Myth. Time-Life Books, 1979. The Old West 26.

Ong, A., and Stephen J. Collier, editors. Global Assemblages: Technology, Politics, and Ethics as Anthropological Problems. Blackwell Publishing, 2007 (2005).

Open Insulin Project. Open Insulin Project. 1 Jan. 2019, openinsulin.org/. Accessed 29 Aug. 2019.

Operskalski, Joachim T., and Aron K. Barbey. "Risk Literacy in Medical DecisionMaking." Science, vol. 352, no. 6284, 2016, pp. 413-414. doi:10.1126/science.aaf7966.

Orphan Black Tumblr. Neolutionism. 8 May 2013, orphanblack.tumblr.com/post/499426 34720/neolutionism-is-a-fictional-term-a-sort-of. Accessed 28 Sep. 2019.

Oudshoorn, Nelly, and André Somers. "Constructing the Digital Patient. Patient Organizations and the Development of Health Websites." Burri and Dumit, Biomedicine as Culture, pp. 205-222.

Owen, Jennifer, and e-NABLE. Enabling the Future. 10 Aug. 2018, enablingthefuture.org 1. Accessed 10 Aug. 2018.

Patterson, Meredith L. A Biopunk Manifesto. I Jan. 2010, maradydd.livejournal.com/4960 85.html. Accessed 1 Jan. 2018. 
Pavone, Vincenzo, and Joanna Goven. Introduction. Bioeconomies: Life, Technology, and Capital in the $21^{\text {st }}$ Century, edited by Vincenzo Pavone and Joanna Goven, Palgrave Macmillan, 2017, pp. 1-22.

Petri, Alexandra E. “D.I.Y. Coronavirus Solutions Are Gaining Steam.” The New York Times, 31 Mar. 2020, www.nytimes.com/2020/03/31/science/coronavirus-masks-equipme nt-crowdsource.html. Accessed 12 Apr. 2021.

Pfeiffer, Sacha, and Harriet A. Washington. The Ethics of Coronavirus Vaccine Trials in Developing Countries. NPR. All Things Considered. Accessed 15 Mar. 2021. www.npr.org/2020/07/04/887325575/the-ethics-of-coronavirus-vaccine-trial s-in-developing-countries.

Piller, Charles, and STAT. "An Anarchist Is Teaching Patients to Make Their Own Medications." Scientific American, 13 Oct. 2017, www.scientificamerican.com/article/ananarchist-is-teaching-patients-to-make-their-own-medications/. Accessed 24 July 2018.

Piore, Adam. The Body Builders: Inside the Science of the Engineered Human. ECCO, 2018.

Pitts, Victoria. In the Flesh: The Cultural Politics of Body Modification. Palgrave Macmillan, 2003.

Pitts-Taylor, Victoria. Cultural Encyclopedia of the Body. Greenwood Pub. Group, 2008.

-. "The Plastic Brain: Neoliberalism and the Neuronal Self." Health (London, England: 1997), vol. 14, no. 6, 2010, pp. 635-652. doi:10.1177/1363459309360796.

—. "Mattering: Feminism, Science and Corporeal Politics." Pitts-Taylor, Mattering: Feminism, Science, pp. 1-20.

—, editor. Mattering: Feminism, Science, and Materialism. New York University Press, 2016. Biopolitics: Medicine, Technoscience, and Health in the Twenty-First Century.

-. "Medicine, Governmentality, and Biopower in Cosmetic Surgery." The Legal, Medical and Cultural Regulation of the Body: Transformation and Transgression, edited by Stephen W. Smith and Ronan Deazley, $2^{\text {nd }}$ ed., Routledge, 2016 (2009), pp. 159-172. Medical Law and Ethics.

-. The Brain's Body: Neuroscience and Corporeal Politics. Duke University Press, 2016.

Plohl, Nejc, and Bojan Musil. "Modeling Compliance with COVID-19 Prevention Guidelines: The Critical Role of Trust in Science." Psychology, Health \& Medicine, 2020, pp. 1-12. doi:10.1080/13548506.2020.1772988.

Poe, Edgar Allan. "The Man That Was Used up: A Tale of the Late Bugaboo and Kickapoo Campaign." The Complete Tales and Poems of Edgar Allan Poe, edited by Edgar Allan Poe, Vintage Books, 1975, pp. 405-412.

Poole, Deborah. Vision, Race, and Modernity: A Visual Economy of the Andean Image World. Princeton University Press, 1997.

Popper, Ben. "Cyborg America: Inside the Strange New World of Basement Body Hackers." The Verge, 8 Aug. 2012, www.theverge.com/2012/8/8/3177438/cyborg-america-b iohackers-grinders-body-hackers. Accessed 5 July 2019.

Powers, Richard. Generosity: An Enhancement. Farrar, Straus and Giroux, 2009.

Promethease. promethease.com/. Accessed 27 July 2018.

Putnam, Robert D. Bowling Alone: The Collapse and Revival of American Community. Simon $\&$ Schuster, 2000. 
Quantified Self Labs. Quantified Self - Self Knowledge Through Numbers. quantifiedself.co m/. Accessed 12 Sep. 2018.

Quartz. Meet the Biohacker Using CRISPR to Teach Everyone Gene Editing. 24 Jan. 2019, ww w.youtube.com/watch?v=4vmHweDC5SY.

Rabinow, Paul. Essays on the Anthropology of Reason. Princeton Univ. Press, 1996.

Rasmussen, Nicolas. On Speed: The Many Lives of Amphetamine. New York University Press; Combined Academic, 2009.

Ratto, Matt, and Megan Boler. Introduction. Boler and Ratto, DIY Citizenship: Critical, pp. 1-22.

Regalado, Antonio. "China's CRISPR Twins Might Have Had Their Brains Inadvertently Enhanced." 21 Feb. 2019, www.technologyreview.com/s/612997/the-crispr-twins-ha d-their-brains-altered/. Accessed 9 May 2019.

-. "This Man Assembled His Own Covid Antibody Tests for Himself and His Friends." MIT Technology Review, 3 May. 2020, www.technologyreview.com/2020/05/03/10009 54/man-diy-covid-antibody-tests-himself/.

Richterich, Annika. "When Open Source Design Is Vital: Critical Making of DIY Healthcare Equipment During the COVID-19 Pandemic." Health Sociology Review: The Journal of the Health Section of the Australian Sociological Association, vol. 29, no. 2, 2020, pp. 158167. doi:10.1080/14461242.2020.1784772.

Robertson, Adi. "I Hacked My Body for a Future That Never Came." The Verge, 21 July 2017, www.theverge.com/2017/7/21/15999544/biohacking-finger-magnet-huma n-augmentation-loss. Accessed 3 July 2019.

Roof, Judith. The Poetics of DNA. University of Minneapolis Press, 2007.

Roosth, Hannah Sophia. Crafting Life: A Sensory Ethnography of Fabricated Biologies. Ph.D. thesis, Massachusetts Institute of Technology, 2010.

Rose, Nikolas. Governing the Soul: The Shaping of the Private Self. $2^{\text {nd }}$ ed. Free Association Books, 1999.

-. "The Politics of Life Itself." Theory, Culture $\&$ Society, vol. 18, no. 6, 2001, pp. 1-30. doi:1 $0.1177 / 02632760122052020$.

-. "Genomic Susceptibility as an Emergent Form of Life? Genetic Testing, Identity, and the Remit of Medicine." Burri and Dumit, Biomedicine as Culture, pp. 141-150.

-. Politics of Life Itself: Biomedicine, Power, and Subjectivity in the Twenty-First Century. Princeton University Press, 2007.

-. "Race, Risk and Medicine in the Age of 'Your Own Personal Genome'." BioSocieties, vol. 3, no. 4, 2008, pp. 423-439. doi:10.1017/S1745855208006339.

-. "Personalized Medicine: Promises, Problems and Perils of a New Paradigm for Healthcare." Procedia - Social and Behavioral Sciences, vol. 77, 2013, pp. 341-352. doi:1 0.1016/j.sbspro.2013.03.092.

Rose, Nikolas, and Carlos Novas. "Biological Citizenship." Ong and Collier, Global Assemblages: Technology, pp. 439-463.

Rosenberg, Buck Clifford. "Scandinavian Dreams: DIY, Democratisation and IKEA." Transformations, 11 - Edges and Centres: Contemporary Experiences and Lifestyle, 2005, www.transformationsjournal.org/wp-content/uploads/2017/01/Rosenberg_T ransformations11.pdf. 
Roy, Arundhati. “The Pandemic Is a Portal'." Financial Times, 3 Apr. 2020, www.ft.com/ content/10d8f5e8-74eb-11ea-95fe-fcd274e920ca. Accessed 8 Mar. 2021.

Samuel, Sigal. "A Celebrity Biohacker Who Sells DIY Gene-Editing Kits Is Under Investigation." Vox, 19 May 2019, www.vox.com/future-perfect/2019/5/19/18629771/biohac king-josiah-zayner-genetic-engineering-crispr. Accessed 5 July 2019.

-. "How Biohackers Are Trying to Upgrade Their Brains, Their Bodies - And Human Nature: 9 Questions About Biohacking You Were Too Embarrassed to Ask." Vox, 25 June 2019, www.vox.com/future-perfect/2019/6/25/18682583/biohacking-transhum anism-human-augmentation-genetic-engineering-crispr. Accessed 5 July 2019.

Sarasin, Philipp. Reizbare Maschinen: Eine Geschichte des Körpers 1765 - 1914. Suhrkamp, 2001.

Schechner, Sam, and Mark Secada. "You Give Apps Sensitive Personal Information. Then They Tell Facebook." The Wall Street Journal, 22 Feb. 2019, www.wsj.com/article s/you-give-apps-sensitive-personal-information-then-they-tell-facebook-11550851 636? $\mathrm{mod}=\mathrm{e} 2 \mathrm{tw}$.

Schmitz, Sigrid. "Materiality's Agency in Technologized Brainbodies." Pitts-Taylor, Mattering: Feminism, Science, pp. 182-203.

Schrödinger, Erwin. What Is Life? The Physical Aspect of the Living Cell. Cambridge University Press, 1944.

Schüll, Natasha Dow. "Data for Life: Wearable Technology and the Design of Self-Care." BioSocieties, vol. 11, no. 3, 2016, pp. 317-333. doi:10.1057/biosoc.2015.47.

Schumacher, Florian. "Von Quantified Self Zur Gesundheit Der Zukunft." Andelfinger and Hänisch, EHealth, pp. 39-51.

Secretary of Defense Donald H. Rumsfeld. DoD News Briefing - Secretary Rumsfeld and Gen. Myers: U.S. Department of Defense, 12 Feb. 2002.

Sedgwick, Eve Kosofsky, et al. Touching Feeling: Affect, Pedagogy, Performativity. Duke University Press, 2002.

Sedgwick, Eve Kosofsky, and Adam Frank. "Shame in the Cybernetic Fold: Reading Silvan Tomkins." Critical Inquiry, vol. 21, no. 2, 1995, pp. 496-522. www.jstor.org/stable /1343932.

Segterra/InsideTracker. Optimize Health, Wellness, Performance Through Blood Tests, Nutrition, Science. www.insidetracker.com/. Accessed 26 July 2018.

Seigworth, Gregory J., and Melissa Gregg. "An Inventory of Shimmers." Gregg and Seigworth, The Affect Theory, pp. 1-25.

Selfhacked/Genius Labs. SelfHacked. 18 Aug. 2018, www.selfhacked.com/. Accessed 18 July 2018.

Selke, Stefan. "Rationale Diskriminierung Durch Lifelogging - Die Optimierung des Individuums auf Kosten des Solidargefüges." Andelfinger and Hänisch, EHealth, pp. 53-71.

Seyfried, Günter, et al. "European Do-It-Yourself (DIY) Biology: Beyond the Hope, Hype and Horror." BioEssays, vol. 36, no. 6, 2014, pp. 548-551. doi:10.1002/bies.201300149.

Sharon, Tamar. "Self-Tracking for Health and the Quantified Self: Re-Articulating Autonomy, Solidarity, and Authenticity in an Age of Personalized Healthcare." Philosophy \& Technology, vol. 30, no. 1, 2017, pp. 93-121. doi:10.1007/s13347-016-0215-5. 
Shaw, Debra Benita. "Posthuman? And Who Are 'We' Anyway?" Science as Culture, vol. 25, no. 2, 2016, pp. 289-295. doi:10.1080/09505431.2015.1120285.

Shea, Elizabeth Parthenia. How the Gene Got Its Groove: Figurative Language, Science, and the Rhetoric of the Real. State University of New York Press, 2008.

Shilling, Chris. The Body and Social Theory. 2nd ed. Sage, 2003.

Siebers, Tobin. Disability Theory. Michigan University Press, 2008.

Silber, Ilana Friedrich. "Towards a Non-Unitary Approach to General Theory." European Journal of Social Theory, vol. 10, no. 2, 2007, pp. 220-232. doi:10.1177/136843100707888 3.

Silverman, Ben, et al., creator. The Biggest Loser. NBC, 2004-2016.

Skloot, Rebecca. The Immortal Life of Henrietta Lacks. First international edition. Crown Publishers, 2010.

Smiles, Samuel. Self-Help; with Illustrations of Character and Conduct. John Murray (publ.), 1859.

Smith, Conrad F., et al. Limitless Biohacking: Gain an Unfair Advantage. The Matterhorn Company, 2017.

Smith, Peter Andrey. "A Do-It-Yourself Revolution in Diabetes Care." New York Times, 22 Feb. 2016, www.nytimes.com/2016/02/23/health/a-do-it-yourself-revolution-in-dia betes-care.html. Accessed 10 Aug. 2018.

SNPedia. 25 July 2018, www.snpedia.com/index.php/SNPedia. Accessed 27 July 2018.

Soderberg, J., and Alessandro Delfanti. "Hacking Hacked! The Life Cycles of Digital Innovation." Science, Technology \& Human Values, vol. 40, no. 5, 2015, pp. 1-6. doi:10.1177 10162243915595091.

Sparke, Penny. As Long as It's Pink: The Sexual Politics of Taste. Pandora, 1995.

Spencer, Amy. DIY: The Rise of Lo-Fi Culture. Marion Boyars, 2008.

Steinberg, Deborah Lynn. Genes and the Bioimaginary. Science, Spectacle, Culture. Routledge, 2015.

Stevens, Hallam. Biotechnology and Society: An Introduction. University of Chicago Press, 2016.

Sung, Dan. "Meet the Grinders: The Humans Using Tech to Live Forever." Wareable, 24 Mar. 2015, www.wareable.com/wearable-tech/meet-the-grinders-implantables. Accessed 7 Aug. 2018.

Sylvia, J. J. "The Biopolitics of Social Distancing." Social Media + Society, vol. 6, no. 3, 2020, pp.1-4. doi:10.1177/2056305120947661.

Tambor, Ellen S., et al. "Mapping the Human Genome: An Assessment of Media Coverage and Public Reaction." Genetics in Medicine, vol. 4, no. 1, 2002, pp. 31-36.

Tanner, Jakob. "Metaphors of Medicine and the Culture of Healing: Historical Perspectives." Burri and Dumit, Biomedicine as Culture, pp. 35-45.

Tanner, Lindsey. "US Kids, Parents Perform DIY Tests for Coronavirus Science | Health News | US News.” US News, 8 Aug. 2020, www.usnews.com/news/health-news/arti cles/2020-08-07/us-kids-parents-perform-diy-tests-for-coronavirus-science.

Tapper, Joan, and Gale Zucker. Craft Activism: People, Ideas, and Projects from the New Community of Handmade and How You Can Join in. $1^{\text {st }}$ ed. Potter Craft, 2011.

Taussig, Karen-Sue. "Genetics and Its Publics. Crafting Genetic Literacy and Identity in the Early Twenty-First Century.” Burri and Dumit, Biomedicine as Culture, pp. 191-204. 
Thacker, Eugene. "What Is Biomedia?" Configurations, vol. 11, no. 1, 2003, pp. 47-79.

-. Biomedia. University of Minnesota Press, 2004.

The Lead with Jake Tapper. CNN, 1 May 2017.

The Nightscout Foundation. FAQs. www.nightscoutfoundation.org/faqs. Accessed 22 Jan. 2020.

-. Our Mission. www.nightscoutfoundation.org/about. Accessed 7 Oct. 2019.

-. Who Are We. www.nightscoutfoundation.org/research. Accessed 7 Oct. 2019.

-. Home. 10 Aug. 2018, www.nightscoutfoundation.org/. Accessed 10 Aug. 2018.

The Nightscout Project. The Nightscout Project-We Are Not Waiting. www.nightscout.info 1. Accessed 10 Aug. 2018.

The ODIN. www.the-odin.com/. Accessed 5 Oct. 2019.

The Swan. FOX, April 7 - December 20, 2004.

Thonis, Sam. Biohackers: A Journey into Cyborg America. uploaded by The Verge, 8 Aug. 2012, YouTube. www.youtube.com/watch?v=KoWIgU7LRcI. Accessed 7 Aug. 2018.

Thrift, Nigel. "Intensities of Feeling: Towards a Spatial Politics of Affect." Geografiska Annaler, Series B: Human Geography, vol. 86, no. 1, 2004, pp. 57-78. doi:10.1111/j.0435-3 684.2004.00154.x.

Till, Chris. "Exercise as Labour: Quantified Self and the Transformation of Exercise into Labour." Societies, vol. 4, no. 3, 2014, pp. 446-462. doi:10.3390/soc4030446.

Tirosh-Samuelson, Hava. Engaging Transhumanism: A Critical Historical Perspective. 1 Jan. 2009, www.metanexus.net/essay/h-engaging-transhumanism-critical-historical-p erspective. Accessed 9 Jan. 2017.

Tocchetti, Sara. DIYbiologists as 'Makers' of Personal Biologies: How MAKE Magazine and Maker Faires Contribute in Constituting Biology as a Personal Technology. 6 Apr. 2017, peerproduction.net/issues/issue-2/peer-reviewed-papers/diybiologists-as-m akers/. Accessed 3 May 2017.

Tocqueville, Alexis de, and Jacob Peter Mayer, editors. Democracy in America. HarperPerennial, 2000.

Toffler, Alvin. The Third Wave. Bantam Books, 1991.

Tomes, Nancy. "Epidemic Entertainments: Disease and Popular Culture in EarlyTwentieth-CenturyAmerica." American Literary History, vol. 14, no. 4, 2002, pp. 625-52. www.jstor.org/stable/3568019.

Tomkins, Silvan. Affect, Imagery, Consciousness: Vol II: The Negative Affects. Springer Publishing, 1963.

Triggs, T. "Scissors and Glue: Punk Fanzines and the Creation of a DIY Aesthetic." Journal of Design History, vol. 19, no. 1, 2006, pp. 69-83. doi:10.1093/jdh/epkoo6.

Turner, Bryan S. The Body \& Society: Explorations in Social Theory. $3^{\text {rd }}$ ed. Sage, 2008.

Turrini, Mauro, and Barbara Prainsack. "Beyond Clinical Utility: The Multiple Values of DTC Genetics.” Applied \& translational genomics, vol. 8, 2016, pp. 4-8. doi:10.1016/j.atg .2016.01.008.

Uno, Gordon E., and Rodger W. Bybee. "Understanding the Dimensions of Biological Literacy." BioScience, vol. 44, no. 8, 1994, pp. 553-557. doi:10.2307/1312283.

van Riper, A. Bowdoin. "What the Public Thinks It Knows About Science: Popular Culture and Its Role in Shaping the Public's Perception of Science and Scientists." EMBO reports, vol. 4, no. 12, 2003, pp. 1104-1107. doi:10.1038/sj.embor.7400040. 
Wald, Priscilla., and Jay Clayton. "Editors' Preface: Genomics in Literature, Visual Arts, and Culture." Literature and Medicine, vol. 26, no. 1, 2007, pp. vi-xvi. doi:10.1353/lm.2 008.0009 .

Waldby, Cathy. The Visible Human Project: Informatic Bodies and Posthuman Medicine. Routledge, 2000.

Washington, Harriet A. Medical Apartheid: The Dark History of Medical Experimentation on Black Americans from Colonial Times to the Present. Anchor books, 2008.

Watkins, Elizabeth Siegel. "How the Pill Became a Lifestyle Drug: The Pharmaceutical Industry and Birth Control in the United States Since 1960." American Journal of Public Health, vol. 102, no. 8, 2012, pp. 1462-1472. doi:10.2105/AJPH.2012.300706.

Watson, Matthew, and Elizabeth Shove. "Product, Competence, Project and Practice: DIY and the Dynamics of Craft Consumption." Journal of Consumer Culture, vol. 8, no. 1, 2008, pp. 69-89. doi:10.1177/1469540507085726.

Wegenstein, Bernadette. Getting Under the Skin: The Body and Media Theory. MIT Press, 2006.

-. The Cosmetic Gaze: Body Modification and the Construction of Beauty. MIT Press, 2012.

Weiss, Richard. The American Myth of Success: From Horatio Alger to Norman Vincent Peale. Illini Books ed. University of Illinois Press, 1969.

Weller, Chris. "A Real-Life Cyborg Says His Sixth-Sense Antenna Will Be Totally Normal in 10 Years." Business Insider, 10 Sep. 2015, www.businessinsider.com/what-its-liketo-be-a-cyborg-2015-9?IR=T. Accessed 7 Aug. 2018.

Wellnicity. About Us. www.wellnicity.com/about-us. Accessed 21 Aug. 2018.

-. Wellnicity: A Better Way to a Healthier You. us.wellnicity.com/. Accessed 26 July 2018.

Wells, Herbert George. "The Limits of Individual Plasticity." H.G. Wells: Early Writings in Science and Science Fiction, edited by Herbert George Wells and Robert M. Philmus, Univ. of Calif. Pr, 1975, pp. 36-39.

Wiggins, Andrea, and John Wilbanks. "The Rise of Citizen Science in Health and Biomedical Research." The American Journal of Bioethics, vol. 19, no. 8, 2019, pp. 3-14. d oi:10.1080/15265161.2019.1619859.

Wikipedia. Biohacking. Accessed 16 Apr. 2021. en.wikipedia.org/wiki/Biohacking.

—. Do-It-Yourself Biology. Accessed 16 Apr. 2021. en.wikipedia.org/wiki/Do-it-yourself_b iology.

Williams, Enita A. Good, Better, Best: The Human Quest for Enhancement: Summary Report of an Invitational Workshop. I June 2006.

Withorn, Ann. "Helping Ourselves: The Limits and Potential of Self-Help." Conrad, Sociology, pp. 510-519.

Wittgenstein, Ludwig, and James Carl Klagge, editors. Philosophical Occasions, 1912 - 1951. Hackett, 1993.

Wohlsen, Marcus. Biopunk: Solving Biotech's Biggest Problems in Kitchens and Garages. Current, 2012, c2011.

Wolf, Gary. "Know Thyself: Tracking Every Facet of Life, from Sleep to Mood to Pain, 24/7/365." Wired, 22 June 2009, www.wired.com/2009/06/lbnp-knowthyself/. Accessed 14 Sep. 2018.

—. "The Data-Driven Life." New York Times, 28 Apr. 2010, www.nytimes.com/2010/05/0 2/magazine/o2self-measurement-t.html. Accessed 10 Apr. 2017. 
Wolinsky, Howard. "Kitchen Biology: The Rise of Do-It-Yourself Biology Democratizes Science, but Is It Dangerous to Public Health and the Environment?" EMBO Reports, vol. 10, no. 7, 2009, pp. 683-685. doi:10.1038/embor.2009.145.

Wyllie, Irvin G. The Self-Made Man in America: The Myth of Rags to Riches. Rutgers University Press, 1954.

Yancy, Clyde W. "COVID-19 and African Americans." JAMA, vol. 323, no. 19, 2020, pp. 1891-92. doi:10.1001/jama.2020.6548.

Zola, Irving Kenneth. "Medicine as an Institution of Social Control." Conrad, Sociology, pp. $470-80$.

Zooniverse. 15 Aug. 2019, www.zooniverse.org/. Accessed 15 Aug. 2019. 


\section{Kulturwissenschaft}

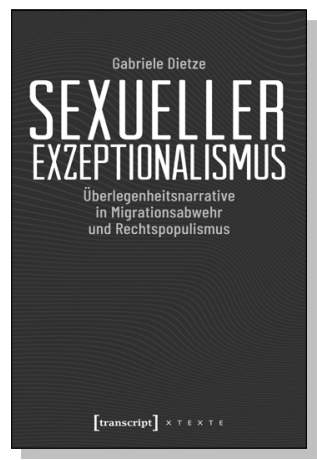

Gabriele Dietze

Sexueller Exzeptionalismus

Überlegenheitsnarrative in Migrationsabwehr und

Rechtspopulismus

2019, 222 S., kart., Dispersionsbindung, 32 SW-Abbildungen $19,99 €(D E), 978-3-8376-4708-2$

E-Book: 17,99 € (DE), ISBN 978-3-8394-4708-6

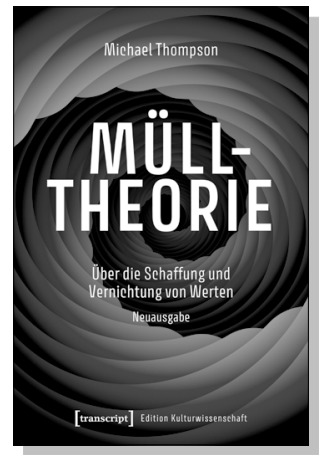

Michael Thompson

Mülltheorie

Über die Schaffung und Vernichtung von Werten

April 2021, 324 S., kart., Dispersionsbindung, 57 SW-Abbildungen

$27,00 €(D E), 978-3-8376-5224-6$

E-Book:

PDF: $23,99 €$ (DE), ISBN 978-3-8394-5224-0

EPUB: $23,99 €(D E)$, ISBN 978-3-7328-5224-6

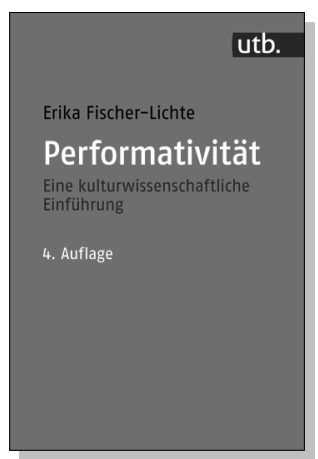

Erika Fischer-Lichte

\section{Performativität}

Eine kulturwissenschaftliche Einführung

April 2021, 274 S., kart., Dispersionsbindung, 3 SW-Abbildungen $22,00 €(D E), 978-3-8376-5377-9$

E-Book:

PDF: $20,99 €$ (DE), ISBN 978-3-8394-5377-3 


\section{Kulturwissenschaft}

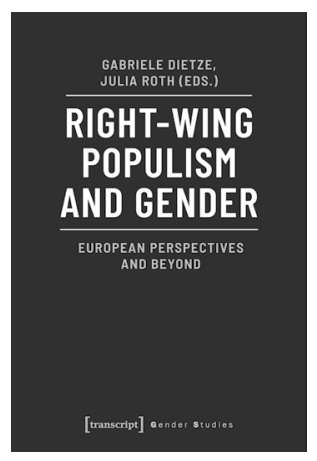

Gabriele Dietze, Julia Roth (eds.)

\section{Right-Wing Populism and Gender}

European Perspectives and Beyond

2020, 286 p., pb., ill.

$35,00 €(D E), 978-3-8376-4980-2$

E-Book:

PDF: $34,99 €$ (DE), ISBN 978-3-8394-4980-6

\section{POP}

Thomas Hecken, Moritz Baßler, Elena Beregow, Robin Curtis, Heinz Drügh, Mascha Jacobs, Annekathrin Kohout, Nicolas Pethes, Miriam Zeh (Hg.)

\section{POP}

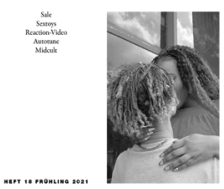

Kultur und Kritik (Jg. 10, 1/2021)

April 2021, 178 S., kart.

$16,80 €(D E), 978-3-8376-5393-9$

E-Book:

PDF: $16,80 €$ (DE), ISBN 978-3-8394-5393-3

[transcript]

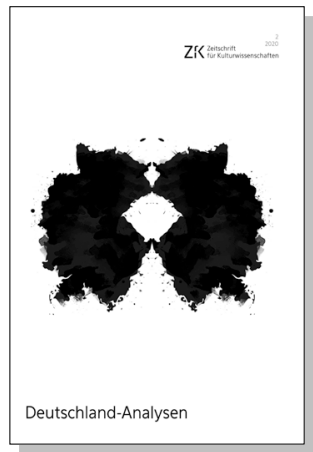

Marcus Hahn, Frederic Ponten (Hg.)

Deutschland-Analysen

Zeitschrift für Kulturwissenschaften, Heft 2/2020

2020, 240 S., kart., Dispersionsbindung, 23 Farbabbildungen $14,99 €(D E), 978-3-8376-4954-3$

E-Book:

PDF: 14,99€ (DE), ISBN 978-3-8394-4954-7 
